\title{
Ru-Catalyzed Chemo- and Enantioselective Hydrogenation of $\beta$ - Diketones Assisted by the Neighboring Heteroatoms
}

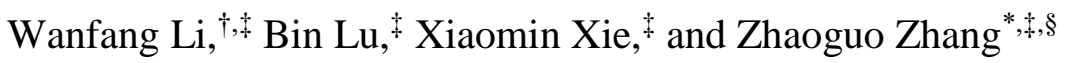 \\ ${ }^{\dagger}$ College of Science, University of Shanghai for Science and Technology, Shanghai 200093, China \\ ${ }^{\ddagger}$ Shanghai Key Laboratory for Molecular Engineering of Chiral Drugs, School of Chemistry and \\ Chemical Engineering, Shanghai Jiao Tong University, 800 Dongchuan Road, Shanghai 200240, China \\ ${ }^{\S}$ State Key Laboratory of Organometallic Chemistry, Shanghai Institute of Organic Chemistry, Chinese \\ Academy of Sciences, 345 Lingling Road, Shanghai 200032, China
}

zhaoguo@sjtu.edu.cn

\section{Table of Contents}

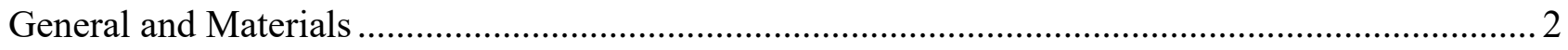

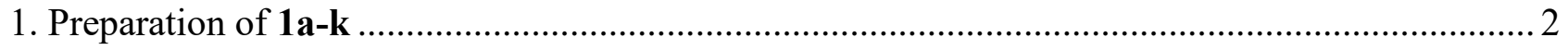

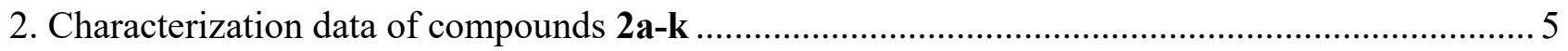

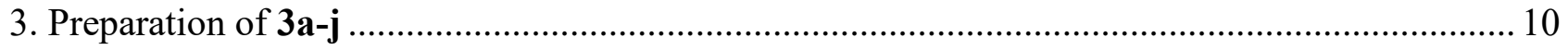

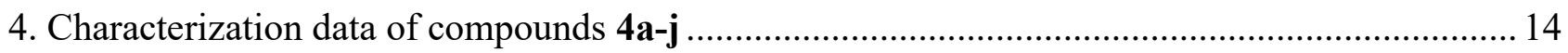

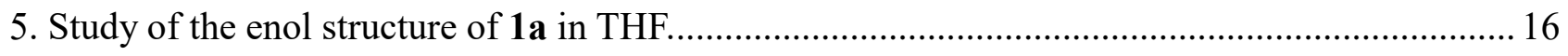

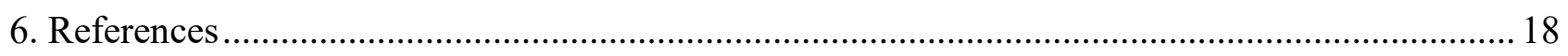

7. NMR spectra of key substrates and all hydrogenation products ............................................. 19

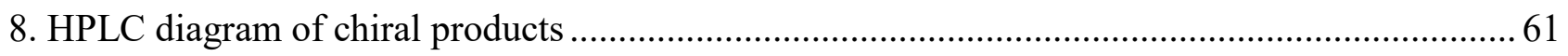




\section{General and Materials}

General: All reactions were carried out under an atmosphere of $\mathrm{N}_{2}$ using standard Schlenk techniques unless otherwise noted. ${ }^{1} \mathrm{H}$ NMR, ${ }^{13} \mathrm{C}$ NMR and ${ }^{19} \mathrm{~F}$ NMR spectra were obtained on a $400 \mathrm{MHz}$ NMR spectrometer. The chemical shifts for ${ }^{1} \mathrm{H}$ NMR were recorded in ppm downfield from tetramethylsilane (TMS) with the solvent resonance as the internal standard. The chemical shifts for ${ }^{13} \mathrm{C}$ NMR were recorded in ppm downfield using the central peak of $\mathrm{CDCl}_{3}(77.00 \mathrm{ppm})$ as the internal standard. Coupling constants $(J)$ are reported in $\mathrm{Hz}$ and refer to apparent peak multiplications. Flash column chromatography was performed on silica gel (300-400 mesh).

Materials: Commercially available reagents were used throughout without further purification other than those detailed below. The solvents used in catalyst preparation and hydrogenation reactions were pretreated by the following procedures: THF and 1,4-dioxane were distilled over sodium benzopheneone ketyl under nitrogen. $\mathrm{CH}_{2} \mathrm{Cl}_{2}$ and DMF was distilled over calcium hydride. EtOH was distilled over magnesium under nitrogen. Acetone, $\mathrm{MeCN}$ and ethyl acetate was distilled over $\mathrm{P}_{2} \mathrm{O}_{5}$ under nitrogen.

\section{Preparation of 1a-k}

(1a) 1-(Benzyloxy)pentane-2,4-dione ${ }^{[1]}$

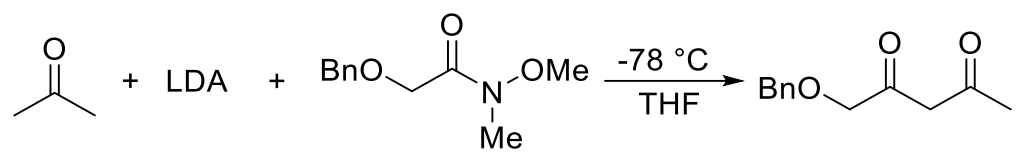

To a stirred solution of LDA (prepared from $6.0 \mathrm{~g}$ of $i \operatorname{Pr}_{2} \mathrm{NH}$ and $32 \mathrm{~mL}$ of $2.4 \mathrm{M} n$-BuLi in hexane, 2.2 eq.) was added acetone $(4.0 \mathrm{~g}, 68.9 \mathrm{mmol})$ dropwise at $-78^{\circ} \mathrm{C}$. The mixture was maintained at $-50{ }^{\circ} \mathrm{C}$ for $1 \mathrm{~h}$ before 2-(benzyloxy)- $N$-methoxy- $N$-methylacetamide (11.5 g, $55.1 \mathrm{mmol}$ ) was added dropwise. Then the mixture was warmed to room temperature and acidified with $10 \% \mathrm{HCl}$ (aq.) to $\mathrm{pH}=4-5$. Then the solvent was removed under reduced pressure and the aqueous phase was extracted with ethyl acetate (20 $\mathrm{mL} \times 3$ ). The combined organic phase was washed with saturated brine and dried over $\mathrm{Na}_{2} \mathrm{SO}_{4}$. Column chromatography (PE/EA = 20:1) gave the product as light yellow liquid $(6.5 \mathrm{~g}, 57 \%) .{ }^{\mathbf{1}} \mathbf{H}$ NMR (400 $\left.\mathrm{MHz}, \mathrm{CDCl}_{3}\right)$ : mixture of keto:enol = 26:74, $\delta 7.42-7.27(\mathrm{~m}, 5 \mathrm{H}), 5.86(\mathrm{~s},-\mathrm{CH}=\mathrm{CO}), 4.59$ and $4.57(\mathrm{~s}$, $\left.\mathrm{PhCH}_{2} \mathrm{O}-, 2 \mathrm{H}\right), 4.09$ and $4.06\left(\mathrm{BnOCH}_{2}-, \mathrm{s}, 2 \mathrm{H}\right), 3.64\left(\mathrm{~s},-\mathrm{COCH}_{2}-\right), 2.23$ and $2.10\left(\mathrm{~s},-\mathrm{CH}_{3}, 3 \mathrm{H}\right) .{ }^{{ }^{13} \mathrm{C}}$ NMR (100 MHz, $\left.\mathrm{CDCl}_{3}\right): \delta 202.8,201.6,191.8,190.7,137.1,128.3,127.9,127.8,127.7,127.6,97.2$, 74.9, 73.4, 73.1, 70.9, 54.1, 30.7, 24.5. 


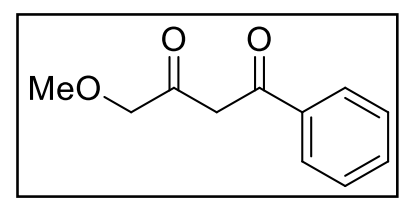

Prepared by the similar procedure to 1 a from $N, 2$-dimethoxy- $N$ methylacetamide. ${ }^{[3]}{ }^{1} \mathbf{H}$ NMR (400 MHz, $\left.\mathrm{CDCl}_{3}\right)$ : mixture of keto:enol = 10:90, $\delta$ 7.95-7.89 (m, 2H), 7.58-7.50 (m, 1H), 7.50-7.41 (m, 2H), $6.49(\mathrm{~s},-\mathrm{CH}=\mathrm{CO})$,

$4.10\left(\mathrm{~s}, \mathrm{OCH}_{2}, 2 \mathrm{H}\right), 3.48$ (s, 3H). ${ }^{13} \mathbf{C} \mathbf{N M R}\left(100 \mathrm{MHz}, \mathrm{CDCl}_{3}\right): \delta 194.3,182.7,134.1,132.3,128.5$, $128.4,128.2,126.9,93.0,74.0,59.0,49.5$.

\section{(1c) 1-Phenoxypentane-2,4-dione ${ }^{[4]}$}<smiles>CC(=O)CC(=O)COc1ccccc1</smiles>

To a suspension of $\mathrm{NaH}$ (60\% in mineral oil, $1.44 \mathrm{~g}, 36.1 \mathrm{mmol})$ in toluene $(100 \mathrm{~mL})$ was added methyl 2-phenoxyacetate ${ }^{[5]}(5.0 \mathrm{~g}, 30.1 \mathrm{mmol})$ in one portion at $80{ }^{\circ} \mathrm{C}$, then acetone $(2.6 \mathrm{~g}, 45.1 \mathrm{mmol})$ was added dropwise and reacted at this temperature for $5 \mathrm{~h}$. The reaction was quenched with $50 \mathrm{~mL}$ of $10 \%$ $\mathrm{HCl}$ (aq.) and the aqueous phase was extracted with ethyl acetate for three times and the combined organic phase was washed with saturated brine and dried over $\mathrm{Na}_{2} \mathrm{SO}_{4}$. Column chromatography (PE/EA = 15:1) gave the product as light yellow liquid (4.1 g, 71\%). ${ }^{1} \mathbf{H} \mathbf{N M R}\left(400 \mathrm{MHz}, \mathrm{CDCl}_{3}\right)$ : mixture of keto:enol = 19:81, $\delta 7.35-7.28(\mathrm{~m}, 2 \mathrm{H}), 7.01(\mathrm{~d}, J=7.4 \mathrm{~Hz}, 1 \mathrm{H}), 6.94-6.88(\mathrm{~m}, 2 \mathrm{H}), 5.90(\mathrm{~s},-\mathrm{CH}=\mathrm{CO})$, 4.60 and $4.57\left(\mathrm{OCH}_{2}, 2 \mathrm{H}\right), 3.76\left(\mathrm{~s}, \mathrm{COCH}_{2}\right), 2.28$ and $2.09(\mathrm{~s}, 3 \mathrm{H}) .{ }^{13} \mathbf{C} \mathbf{N M R}\left(100 \mathrm{MHz}, \mathrm{CDCl}_{3}\right): \delta$ 201.2, 191.2, 190.1, 157.6, 129.4, 129.3, 121.3, 114.3, 114.2, 97.0, 72.3, 68.5, 53.7, 30.5, 24.0.

$\mathbf{1}(\mathbf{d}-\mathbf{k})$ were prepared from various aryl methyl ketones with ethyl 2-benzoxyacetate ${ }^{[6]}$ as $\mathbf{1 c}$.

(1d) 4-(Benzyloxy)-1-phenylbutane-1,3-dione

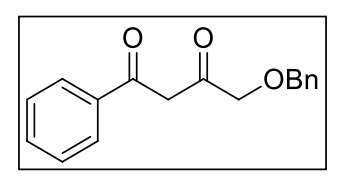

Light yellow liquid. ${ }^{1} \mathbf{H}$ NMR (400 MHz, $\mathrm{CDCl}_{3}$ ): mixture of keto:enol = 11:89, $\delta 7.98-7.84(\mathrm{~m}, 2 \mathrm{H}), 7.53(\mathrm{dd}, J=5.0,3.8 \mathrm{~Hz}, 1 \mathrm{H}), 7.52-7.41(\mathrm{~m}, 2 \mathrm{H}), 7.42-7.26$ $(\mathrm{m}, 4 \mathrm{H}), 7.23-7.11(\mathrm{~m}, 1 \mathrm{H}), 6.55(\mathrm{~s},-\mathrm{CH}=\mathrm{CO}), 4.66(\mathrm{~s}, 2 \mathrm{H}), 4.56(\mathrm{~s}, 1 \mathrm{H}), 4.18(\mathrm{~s}$, 2H). ${ }^{13} \mathrm{C} \mathrm{NMR}\left(100 \mathrm{MHz}, \mathrm{CDCl}_{3}\right): \delta 194.5,183.0,137.2,134.4,132.5,128.6,128.5,128.2,128.0,127.8$, 127.1, 93.4, 73.4, 71.7. HRMS Calculated for $\mathrm{C}_{17} \mathrm{H}_{16} \mathrm{O}_{3} \mathrm{Na}(\mathrm{M}+\mathrm{Na})^{+}:$291.0997, found: 291.0994 . 
(1e) 4-(Benzyloxy)-1-(4-methoxyphenyl)butane-1,3-dione

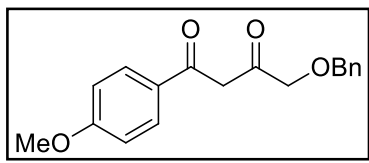

Light yellow liquid. ${ }^{1} \mathbf{H}$ NMR (400 MHz, $\mathrm{CDCl}_{3}$ ): mixture of keto:enol = 15:85, $\delta 7.89(\mathrm{~d}, J=9.0 \mathrm{~Hz}, 2 \mathrm{H}), 7.42-7.24(\mathrm{~m}, 5 \mathrm{H}), 6.95(\mathrm{~d}, J=9.0 \mathrm{~Hz}, 2 \mathrm{H}), 6.48(\mathrm{~s},-$ $\mathrm{CH}=\mathrm{CO}), 4.65$ and $4.56\left(\mathrm{~s}, \mathrm{PhCH}_{2} \mathrm{O}, 2 \mathrm{H}\right), 4.17$ and $4.12\left(\mathrm{~s}, \mathrm{OCH}_{2} \mathrm{CO}, 2 \mathrm{H}\right), 3.87$ (s, OMe, 3H). ${ }^{13} \mathrm{C}$ NMR (100 MHz, $\left.\mathrm{CDCl}_{3}\right): \delta 192.1,183.6,163.1,137.2,130.7,129.1,128.4,128.3$, 127.8, 127.7, 126.8, 113.8, 92.4, 74.9, 73.3, 73.2, 71.3, 55.3, 49.6. HRMS Calculated for $\mathrm{C}_{18} \mathrm{H}_{19} \mathrm{O}_{4}$ $(\mathrm{M}+\mathrm{H})^{+}:$299.1283, found: 299.1280.

\section{(1f) 4-(Benzyloxy)-1-(4-(trifluoromethyl)phenyl)butane-1,3-dione}

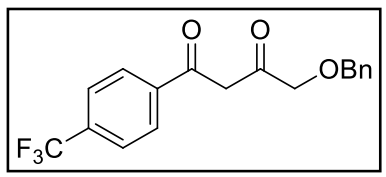

Light yellow solid, m.p.: $74.7-78.3{ }^{\circ} \mathrm{C} .{ }^{1} \mathbf{H}$ NMR (400 MHz, $\left.\mathrm{CDCl}_{3}\right)$ : mixture of keto:enol $=15: 85, \delta 8.00(\mathrm{~d}, J=8.2 \mathrm{~Hz}, 2 \mathrm{H}), 7.71(\mathrm{~d}, J=8.2 \mathrm{~Hz}, 2 \mathrm{H}), 7.47-$ $7.27(\mathrm{~m}, 5 \mathrm{H}), 6.57$ (s, CH=CO), $4.66(\mathrm{~s}, 2 \mathrm{H}), 4.19$ (s, 2H). ${ }^{13} \mathrm{C} \mathrm{NMR}(100 \mathrm{MHz}$, $\left.\mathrm{CDCl}_{3}\right): \delta 196.0,180.4,137.6,137.0,133.8,133.5,129.3,128.8,128.6,128.1,127.8,127.3,127.2,125.6$, 125.5, 125.0, 94.1, 73.5, 71.8. HRMS Calculated for $\mathrm{C}_{18} \mathrm{H}_{15} \mathrm{~F}_{3} \mathrm{O}_{3} \mathrm{Na}(\mathrm{M}+\mathrm{Na})^{+}:$359.0871, found: 359.0871 .

\section{(1g) 4-(Benzyloxy)-1-(2-chlorophenyl)butane-1,3-dione}

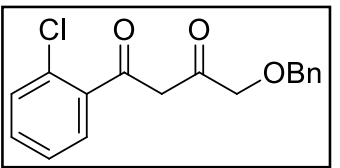

Light yellow liquid. ${ }^{1} \mathbf{H}$ NMR $\left(400 \mathrm{MHz}, \mathrm{CDCl}_{3}\right)$ : mixture of keto:enol = 9:91, $\delta$ 7.95-7.27 (m, 9H), 6.55 (s, -CH=CO), 6.44 (s, 1H), 4.64 (s, 2H), 4.19 (s, 2H). ${ }^{13} \mathrm{C}$

NMR (100 MHz, $\left.\mathrm{CDCl}_{3}\right): \delta 193.5,183.8,137.0,134.9,132.4,131.8,131.7,130.6$, 130.0, 128.5, 128.4, 127.9, 127.8, 127.7, 127.0, 126.8, 98.8, 93.4, 73.3, 71.3, 53.6. HRMS Calculated for $\mathrm{C}_{17} \mathrm{H}_{15} \mathrm{ClO}_{3} \mathrm{Na}(\mathrm{M}+\mathrm{Na})^{+}:$325.0607, found: 325.0600 .

\section{(1h) 4-(Benzyloxy)-1-(o-tolyl)butane-1,3-dione}

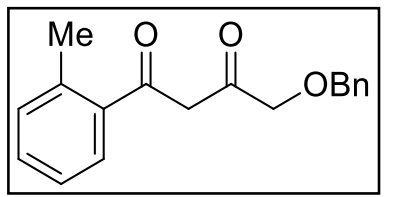

Light yellow liquid. ${ }^{1} \mathbf{H}$ NMR (400 MHz, $\mathrm{CDCl}_{3}$ ): mixture of keto:enol = 17:83, $\delta 7.69-7.15$ (m, 9H), 6.23 (s, CH=CO), 4.64 (s, 2H), 4.17 (s, 2H), 2.59 and 2.51

(s 3H). ${ }^{13}$ C NMR (100 MHz, $\left.\mathrm{CDCl}_{3}\right): \delta$ 193.6, 187.6, 137.2, 135.3, 131.0, 131.4, $130.8,129.3,128.5,127.9,127.7,125.7,125.6,97.6,73.3,71.5,20.8$. HRMS Calculated for $\mathrm{C}_{18} \mathrm{H}_{18} \mathrm{O}_{3} \mathrm{Na}$ $(\mathrm{M}+\mathrm{Na})^{+}:$305.1154, found: 305.1155 . 


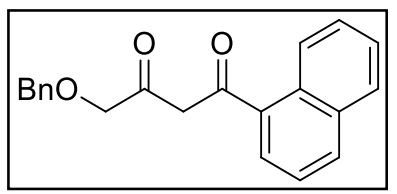

Yellow oil. ${ }^{1} \mathbf{H}$ NMR (400 MHz, $\mathrm{CDCl}_{3}$ ): mixture of keto:enol $=20: 80, \delta 8.47$ $(\mathrm{s}, 1 \mathrm{H}), 7.97-7.85(\mathrm{~m}, 4 \mathrm{H}), 7.66-7.49(\mathrm{~m}, 3 \mathrm{H}), 7.44-7.33(\mathrm{~m}, 4 \mathrm{H}), 6.69(\mathrm{~s}$, $\mathrm{CH}=\mathrm{CO}), 4.69$ and 4.57 (s, 2H), 4.30 (s, 1H), 4.22 (s, 2H). ${ }^{13} \mathbf{C ~ N M R}(100 \mathrm{MHz}$, $\left.\mathrm{CDCl}_{3}\right): \delta 194.5,182.8,137.2,135.3,132.6,131.6,129.6,129.3,128.8,128.6,128.4,128.1,128.0,127.9$, 127.7, 127.4, 126.7, 126.4, 126.2, 126.0, 123.1, 93.8, 73.5, 71.8, 50.0. HRMS Calculated for $\mathrm{C}_{21} \mathrm{H}_{18} \mathrm{O}_{3} \mathrm{Na}$ $(\mathrm{M}+\mathrm{Na})^{+}:$341.1154, found: 341.1147 .

\section{(1j) 4-(Benzyloxy)-1-(naphthalen-2-yl)butane-1,3-dione}

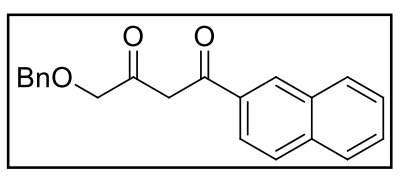

Light yellow solid, m.p.: 41.2-42.4 ${ }^{\circ} \mathrm{C} .{ }^{1} \mathbf{H}$ NMR (400 MHz, $\left.\mathrm{CDCl}_{3}\right)$ : mixture of keto:enol $=25: 75, \delta 8.56(\mathrm{~d}, J=8.4 \mathrm{~Hz}, 1 \mathrm{H}), 7.96-7.87(\mathrm{~m}, 2 \mathrm{H}), 7.80(\mathrm{dd}$, $J=7.2,1.2 \mathrm{~Hz}, 1 \mathrm{H}), 7.65-7.47(\mathrm{~m}, 3 \mathrm{H}), 7.51-7.22(\mathrm{~m}, 5 \mathrm{H}), 6.47$ (s, $-\mathrm{CH}=\mathrm{CO})$,

4.68 and 4.59 (s, 2H), 4.30 and 4.26 (s, 2H). $\left.{ }^{13} \mathbf{C ~ N M R ~ ( 1 0 0 ~ M H z , ~} \mathrm{CDCl}_{3}\right): \delta 192.8,187.8,137.1,133.7$, 133.6, 131.8, 130.0, 128.4, 128.3, 127.9, 127.8, 127.7, 127.3, 127.2, 126.3, 125.4, 124.6, 98.5, 73.3, 71.3, 53.0. HRMS Calculated for $\mathrm{C}_{21} \mathrm{H}_{19} \mathrm{O}_{3}(\mathrm{M}+\mathrm{H})^{+}: 319.1334$, found: 319.1328 .

(1k) 4-(Benzyloxy)-1-(furan-2-yl)butane-1,3-dione

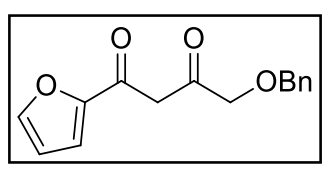

Yellow liquid. ${ }^{1} \mathbf{H}$ NMR (400 MHz, $\left.\mathrm{CDCl}_{3}\right)$ : mixture of keto:enol = 25:75, $\delta 7.59$ $(\mathrm{dd}, J=1.6,0.8 \mathrm{~Hz}, 1 \mathrm{H}), 7.41-7.25(\mathrm{~m}, 5 \mathrm{H}), 7.21-7.15(\mathrm{~m}, 1 \mathrm{H}), 6.59-6.48(\mathrm{~m}, 1 \mathrm{H})$, $6.41(\mathrm{~s}, \mathrm{CH}=\mathrm{CO}), 4.64$ and $4.56(\mathrm{~s}, 2 \mathrm{H}), 4.18\left(\mathrm{~s}, \mathrm{COCH}_{2}\right), 4.15$ and $4.03\left(\mathrm{~s}, \mathrm{OCH}_{2}\right.$, 2H). ${ }^{13} \mathrm{C}$ NMR (100 MHz, $\left.\mathrm{CDCl}_{3}\right): \delta 189.8,175.5,149.8,146.8,146.2,137.0,129.3,128.3,128.1,127.8$, 127.7, 118.2, 116.0, 112.4, 93.1, 70.5, 49.6. HRMS Calculated for $\mathrm{C}_{15} \mathrm{H}_{14} \mathrm{O}_{4} \mathrm{Na}(\mathrm{M}+\mathrm{Na})^{+}:$281.0790, found: 281.0791 .

\section{Characterization data of compounds $2 a-k$}

Typical procedure for asymmetric hydrogenation reactions: To a $20 \mathrm{~mL}$ Schlenk tube were added $\left[\mathrm{Ru} \text { (benzene) } \mathrm{Cl}_{2}\right]_{2}(5.0 \mathrm{mg}, 10.0 \mu \mathrm{mol})$ and $(S)$-SunPhos $(15.1 \mathrm{mg}, 22.6 \mu \mathrm{mol})$. The tube was vacuumed and purged with nitrogen three times before addition of freshly distilled and freeze-and-thaw degassed $\mathrm{EtOH} / \mathrm{CH}_{2} \mathrm{Cl}_{2}(1 \mathrm{~mL} / 1 \mathrm{~mL})$. The resulting mixture was heated at $50{ }^{\circ} \mathrm{C}$ for $1 \mathrm{~h}$ and then the solvent was removed under vacuum to give the catalyst as a brownish yellow solid. The catalyst was dissolved in degassed THF (10 mL) and then the solution was equally divided into 5 vials. Then the vials were taken into an autoclave. The autoclave was purged three times with $\mathrm{H}_{2}$ and the required pressure of $\mathrm{H}_{2}$ was set. Then the autoclave was stirred under specified reaction conditions. After being cooled to ambient 
temperature and careful release of the hydrogen, the autoclave was opened, and the solvent was evaporated. The enantiomeric excess was determined by HPLC after passing the samples through a short pad of silica gel eluted with petroleum ether and ethyl acetate.

Table S1. Screening of the Other Solvents

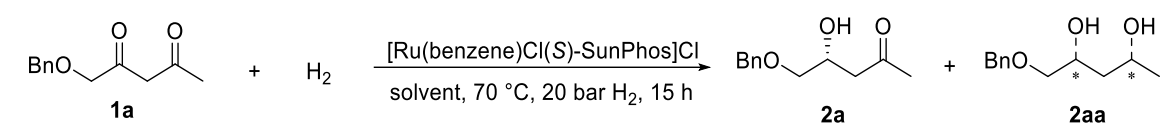

\begin{tabular}{|c|c|c|c|c|c|}
\hline \multirow{2}{*}{ entry } & \multirow{2}{*}{ solvent } & conversion & \multicolumn{2}{|c|}{ 2a } & 2aa \\
\cline { 4 - 6 } & & $(\%)$ & isolated yield (\%) & ee (\%) & isolated yield (\%) \\
\hline 1 & MeCN & $<2$ & -- & -- & -- \\
\hline 2 & DMF & $<2$ & -- & -- & -- \\
\hline 3 & dioxane & 80 & 76 & 94.0 & -- \\
\hline 4 & EtOAc & 19 & 17 & 92.8 & -- \\
\hline 5 & EtOH & 99 & -- & -- & 90 \\
\hline 6 & DCM & 95 & -- & -- & 89 \\
\hline 7 & Acetone & 95 & -- & - & \\
\hline
\end{tabular}

\section{(2a) (R)-5-(Benzyloxy)-4-hydroxypentan-2-one ${ }^{[7]}$}

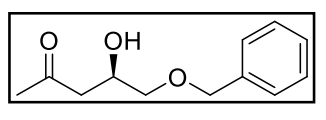

Purified by flash column chromatography $(\mathrm{PE} / \mathrm{EA}=5: 1)$ as light yellow liquid (97 mg, 93\%). ${ }^{1} \mathbf{H}$ NMR (400 MHz, $\left.\mathrm{CDCl}_{3}\right): \delta 7.39-7.27(\mathrm{~m}, 5 \mathrm{H}), 4.55(\mathrm{~d}, J=1.3 \mathrm{~Hz}$, 2H), 4.26 (dd, $J=7.0,4.6 \mathrm{~Hz}, 1 \mathrm{H}), 3.47$ (qd, $J=9.6,5.4 \mathrm{~Hz}, 2 \mathrm{H}), 2.97$ (d, $J=4.0 \mathrm{~Hz}, 1 \mathrm{H}), 2.72-2.59$ (m, 2H), 2.18 (s, 3H). ${ }^{13} \mathbf{C}$ NMR (100 MHz, $\left.\mathrm{CDCl}_{3}\right): \delta 207.9,137.5,127.9,127.2,73.0,72.7,66.1,46.4$, 30.2. HPLC (Chiralcel OJ-H column, hexane $/{ }^{i} \mathrm{PrOH}=94 / 6,0.9 \mathrm{~mL} \mathrm{~min}^{-1}, 220 \mathrm{~nm}$ ): $\mathrm{t}_{1}=25.9 \mathrm{~min}, \mathrm{t}_{2}=$ 27.6 min. $[\alpha]_{\mathrm{D}}^{25}=+16.4\left(\mathrm{c} 0.59, \mathrm{CH}_{2} \mathrm{Cl}_{2}\right)$; Reference value: ${ }^{[7]}[\alpha]_{\mathrm{D}}^{\mathrm{rt}}=-13.8\left(c 0.58, \mathrm{CH}_{2} \mathrm{Cl}_{2}\right)$ for $(S)-5 \mathbf{a}$ with $98 \%$ ee. 


\section{(2aa) anti-1-(Benzyloxy)pentane-2,4-diol ${ }^{[8]}$}

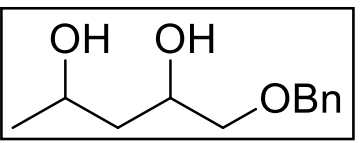

Purified by flash column chromatography $(\mathrm{PE} / \mathrm{EA}=5: 1)$ as light yellow liquid (42 mg, 40\%). The anti-configuration was determined by comparing the ${ }^{13} \mathrm{C} \mathrm{NMR}$ data with the literature value. ${ }^{[8]}{ }^{1} \mathbf{H}$ NMR $\left(400 \mathrm{MHz}, \mathrm{CDCl}_{3}\right): \delta 7.45-7.17(\mathrm{~m}$, $5 \mathrm{H}), 4.56$ (s, 2H), 4.15-4.09 (m, 2H), 3.72-3.87 (m, 0.4H), 3.51-3.40 (m, 2H), 1.64-1.51 (m, 2H), 1.22 $(\mathrm{d}, J=6.4 \mathrm{~Hz}, 3 \mathrm{H}) .{ }^{13} \mathrm{C} \mathbf{N M R}\left(100 \mathrm{MHz}, \mathrm{CDCl}_{3}\right): \delta 137.7,128.2,127.6,74.4,73.1,67.5,64.5,40.8$, 23.4 .

\section{(2b) 3-Hydroxy-4-methoxy-1-phenylbutan-1-one}

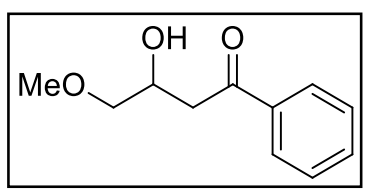

Purified by flash column chromatography $(\mathrm{PE} / \mathrm{EA}=4: 1)$ as light yellow liquid (185 mg, 95\%). ${ }^{1} \mathbf{H}$ NMR (400 MHz, $\left.\mathrm{CDCl}_{3}\right): \delta 8.01-7.93$ (m, 2H), 7.62-7.55 (m, $1 \mathrm{H}), 7.50-7.42(\mathrm{~m}, 2 \mathrm{H}), 4.49-4.35(\mathrm{~m}, 1 \mathrm{H}), 3.50(\mathrm{t}, J=5.2 \mathrm{~Hz}, 2 \mathrm{H}), 3.41(\mathrm{~s}, 3 \mathrm{H})$, $3.21(\mathrm{~d}, J=1.0 \mathrm{~Hz}, 1 \mathrm{H}), 3.20(\mathrm{~d}, J=5.6 \mathrm{~Hz}, 2 \mathrm{H}) .{ }^{13} \mathbf{C ~ N M R}\left(100 \mathrm{MHz}, \mathrm{CDCl}_{3}\right): \delta 199.7,136.6,133.4$, 128.5, 128.0, 75.7, 66.7, 59.1, 41.7. HPLC (ChiralPak AS-H column, hexane ${ }^{i} \mathrm{PrOH}=88 / 12,0.8 \mathrm{~mL}$ $\left.\min ^{-1}, 220 \mathrm{~nm}\right): \mathrm{t}_{1}=13.9 \mathrm{~min}, \mathrm{t}_{2}=23.6 \mathrm{~min}$. HRMS Calculated for $\mathrm{C}_{11} \mathrm{H}_{14} \mathrm{O}_{3} \mathrm{Na}(\mathrm{M}+\mathrm{Na})^{+}: 217.0841$, found: 217.0835 .

\section{(2c) (R)-4-Hydroxy-5-phenoxypentan-2-one ${ }^{[9]}$}

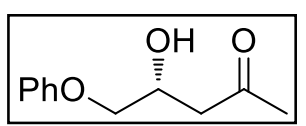

Purified by flash column chromatography $(\mathrm{PE} / \mathrm{EA}=5: 1)$ as white solid $(178 \mathrm{mg}$, 92\%), m.p.: 53.3-55.3 ${ }^{\circ} \mathrm{C}^{1}{ }^{1} \mathbf{H}$ NMR $\left(400 \mathrm{MHz}, \mathrm{CDCl}_{3}\right): \delta 7.34-7.20(\mathrm{~m}, 2 \mathrm{H}), 7.01-$ $6.93(\mathrm{~m}, 1 \mathrm{H}), 6.93-6.86(\mathrm{~m}, 2 \mathrm{H}), 4.50-4.35(\mathrm{~m}, 1 \mathrm{H}), 3.97$ (d, $J=5.4 \mathrm{~Hz}, 2 \mathrm{H}), 3.14$ (dd, $J=4.2,2.8 \mathrm{~Hz}$, $1 \mathrm{H}), 2.80(\mathrm{~d}, J=6.0 \mathrm{~Hz}, 2 \mathrm{H}), 2.23(\mathrm{~s}, 3 \mathrm{H}) .{ }^{13} \mathrm{C} \mathrm{NMR}\left(100 \mathrm{MHz}, \mathrm{CDCl}_{3}\right): \delta 208.3,158.2,129.3,120.8$, 114.3, 70.6, 65.9, 46.4, 30.5. HPLC (Chiralcel OJ-H column, hexane $/{ }^{i} \mathrm{PrOH}=94 / 6,0.9 \mathrm{~mL} \mathrm{~min}{ }^{-1}, 220$ $\mathrm{nm}): \mathrm{t}_{1}=28.4 \mathrm{~min}, \mathrm{t}_{2}=31.5 \mathrm{~min}$. HRMS Calculated for $\mathrm{C}_{11} \mathrm{H}_{14} \mathrm{O}_{3} \mathrm{Na}(\mathrm{M}+\mathrm{Na})^{+}: 271.0841$, found: 271.0842 .

\section{(2d) (R)-4-(Benzyloxy)-3-hydroxy-1-phenylbutan-1-one ${ }^{[7]}$}

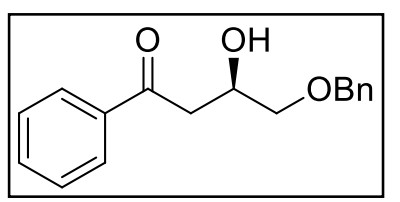

Purified by flash column chromatography $(\mathrm{PE} / \mathrm{EA}=5: 1)$ as light yellow oil (254 mg, 94\%). ${ }^{\mathbf{1}} \mathbf{H}$ NMR (400 MHz, $\left.\mathrm{CDCl}_{3}\right): \delta 8.00-7.91(\mathrm{~m}, 2 \mathrm{H}), 7.57(\mathrm{~d}, J$ $=7.4 \mathrm{~Hz}, 1 \mathrm{H}), 7.47(\mathrm{dd}, J=10.8,4.5 \mathrm{~Hz}, 2 \mathrm{H}), 7.40-7.27(\mathrm{~m}, 5 \mathrm{H}), 4.59(\mathrm{~d}, J=$ $2.0 \mathrm{~Hz}, 2 \mathrm{H}), 4.50-4.40(\mathrm{~m}, 1 \mathrm{H}), 3.59$ (qd, $J=9.6,5.2 \mathrm{~Hz}, 2 \mathrm{H}), 3.22(\mathrm{~d}, J=0.8 \mathrm{~Hz}, 1 \mathrm{H}), 3.20$ (s, 2H). ${ }^{13} \mathrm{C}$ NMR $\left(100 \mathrm{MHz}, \mathrm{CDCl}_{3}\right): \delta 199.5,137.8,136.6,133.2,128.4,128.2,128.0,127.6,73.2,66.9,41.7$. HPLC (Chiralcel AD-H column, hexane $/{ }^{i} \mathrm{PrOH}=88 / 12,0.8 \mathrm{~mL} \mathrm{~min}^{-1}, 220 \mathrm{~nm}$ ): $\mathrm{t}_{1}=17.5 \mathrm{~min}, \mathrm{t}_{2}=19.2$ 
$\min .[\alpha]_{\mathrm{D}}^{25}=+24.6\left(c 0.47, \mathrm{CH}_{2} \mathrm{Cl}_{2}\right)$; Reference value: ${ }^{[7]}[\alpha]_{\mathrm{D}}^{\mathrm{rt}}=-21.4\left(\mathrm{c} 0.46, \mathrm{CH}_{2} \mathrm{Cl}_{2}\right)$ for $(S)-2 \mathrm{~d}$ with $98 \%$ ee.

(2e) 4-(Benzyloxy)-3-hydroxy-1-(4-methoxyphenyl)butan-1-one

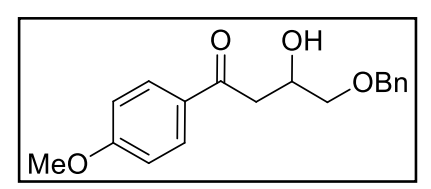

Purified by flash column chromatography $(\mathrm{PE} / \mathrm{EA}=4: 1)$ as light yellow liquid $(274 \mathrm{mg}, 91 \%) .{ }^{1} \mathbf{H}$ NMR $\left(400 \mathrm{MHz}, \mathrm{CDCl}_{3}\right): \delta 8.02-7.85(\mathrm{~m}, 2 \mathrm{H}), 7.38-7.26$ $(\mathrm{m}, 5 \mathrm{H}), 7.01-6.89(\mathrm{~m}, 2 \mathrm{H}), 4.58(\mathrm{~d}, J=1.6 \mathrm{~Hz}, 2 \mathrm{H}), 3.87(\mathrm{~s}, 3 \mathrm{H}), 3.63-3.53$ $(\mathrm{m}, 2 \mathrm{H}), 3.16(\mathrm{~s}, 1 \mathrm{H}), 3.15(\mathrm{~d}, J=1.0 \mathrm{~Hz}, 1 \mathrm{H}) .{ }^{13} \mathbf{C} \mathbf{N M R}\left(100 \mathrm{MHz}, \mathrm{CDCl}_{3}\right): \delta 197.9,163.4,137.7$, 130.2, 129.6, 128.0, 127.4, 127.3, 113.4, 73.2, 73.0, 66.8, 55.1, 41.2, 29.4. HPLC (ChiralPak IB-3 column, hexane $\left./{ }^{i} \operatorname{PrOH}=85 / 15,0.8 \mathrm{~mL} \mathrm{~min}^{-1}, 220 \mathrm{~nm}\right): \mathrm{t}_{1}=13.4 \mathrm{~min}, \mathrm{t}_{2}=14.5 \mathrm{~min}$. HRMS Calculated for $\mathrm{C}_{18} \mathrm{H}_{21} \mathrm{O}_{4}(\mathrm{M}+\mathrm{H})^{+}:$301.1440, found: 301.1444 .

\section{(2f) 4-(Benzyloxy)-3-hydroxy-1-(4-(trifluoromethyl)phenyl)butan-1-one}

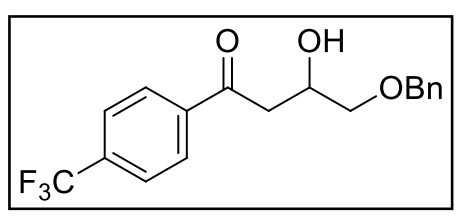

Purified by flash column chromatography $(\mathrm{PE} / \mathrm{EA}=5: 1)$ as light yellow liquid (305 mg, 90\%). ${ }^{1} \mathbf{H}$ NMR (400 MHz, $\left.\mathrm{CDCl}_{3}\right): \delta 8.05(\mathrm{~d}, J=8.0 \mathrm{~Hz}$, 2H), $7.73(\mathrm{~d}, J=8.2 \mathrm{~Hz}, 2 \mathrm{H}), 7.39-7.27(\mathrm{~m}, 5 \mathrm{H}), 4.58(\mathrm{~d}, J=2.8 \mathrm{~Hz}, 2 \mathrm{H})$, $4.51-4.40(\mathrm{~m}, 1 \mathrm{H}), 3.59(\mathrm{qd}, J=9.6,5.3 \mathrm{~Hz}, 2 \mathrm{H}), 3.29-3.16(\mathrm{~m}, 2 \mathrm{H}), 3.03$ $(\mathrm{d}, J=4.2 \mathrm{~Hz}, 1 \mathrm{H}) .{ }^{13} \mathrm{C}$ NMR $\left(100 \mathrm{MHz}, \mathrm{CDCl}_{3}\right): \delta 198.6,139.4,137.7,134.4,128.4,128.4,127.8$, 127.8, 126.0, 125.7, 125.6, 125.2, 124.8, 73.4, 73.1, 66.9, 42.2. HPLC (Chiralcel AD-H column, hexane $\left./{ }^{i} \mathrm{PrOH}=88 / 12,0.8 \mathrm{~mL} \mathrm{~min}{ }^{-1}, 220 \mathrm{~nm}\right): \mathrm{t}_{1}=14.9 \mathrm{~min}, \mathrm{t}_{2}=17.7 \mathrm{~min}$. HRMS Calculated for $\mathrm{C}_{18} \mathrm{H}_{17} \mathrm{~F}_{3} \mathrm{O}_{2} \mathrm{Na}(\mathrm{M}+\mathrm{Na})^{+}:$361.1027, found: 361.1030 .

\section{(2gg) 4-(Benzyloxy)-1-(2-chlorophenyl)butane-1,3-diol}

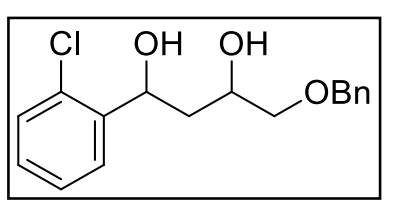

Purified by flash column chromatography $(\mathrm{PE} / \mathrm{EA}=5: 1)$ as light yellow oil (282 mg, 92\%). ${ }^{1} \mathbf{H}$ NMR $\left(400 \mathrm{MHz}, \mathrm{CDCl}_{3}\right): \delta 8.00-7.11(\mathrm{~m}, 9 \mathrm{H}), 5.34(\mathrm{~d}, J=9.8$ $\mathrm{Hz}, 1 \mathrm{H}), 4.57(\mathrm{~d}, J=10.4 \mathrm{~Hz}, 2 \mathrm{H}), 4.24(\mathrm{~s}, 1 \mathrm{H}), 3.98(\mathrm{~s}, 1 \mathrm{H}), 3.59-3.37$ (m, 2H), 3.24-3.16 (m, 1H), $1.92(\mathrm{~d}, J=14.6 \mathrm{~Hz}, 1 \mathrm{H}), 1.68(\mathrm{dt}, J=14.4,10.0 \mathrm{~Hz}, 2 \mathrm{H}) .{ }^{13} \mathrm{C} \mathrm{NMR}(100 \mathrm{MHz}$, $\left.\mathrm{CDCl}_{3}\right): \delta 141.6,137.6,131.1,129.0,128.5,128.3,128.2,128.0,127.7,127.0,74.2,73.2,73.2,71.1$, 70.6, 68.0, 66.9, 41.7, 39.9. HRMS Calculated for $\mathrm{C}_{17} \mathrm{H}_{19} \mathrm{ClO}_{3} \mathrm{Na}(\mathrm{M}+\mathrm{Na})^{+}$: 329.0920, found: 329.0925 . 


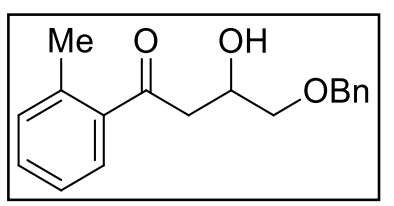

Purified by flash column chromatography (PE/EA = 5:1) as light yellow oil (271 mg, 95\%). ${ }^{1} \mathbf{H}$ NMR $\left(400 \mathrm{MHz}, \mathrm{CDCl}_{3}\right): \delta 7.67(\mathrm{~d}, J=7.8 \mathrm{~Hz}, 1 \mathrm{H}), 7.47-7.17$ $(\mathrm{m}, 8 \mathrm{H}), 4.58(\mathrm{~d}, J=2.0 \mathrm{~Hz}, 2 \mathrm{H}), 4.42(\mathrm{~s}, 1 \mathrm{H}), 3.56(\mathrm{dd}, J=5.4,4.2 \mathrm{~Hz}, 2 \mathrm{H})$, $3.18(\mathrm{~d}, J=3.6 \mathrm{~Hz}, 1 \mathrm{H}), 3.16-3.09(\mathrm{~m}, 2 \mathrm{H}), 2.50$ (s, 3H). ${ }^{13} \mathrm{C}$ NMR $(100 \mathrm{MHz}$, $\left.\mathrm{CDCl}_{3}\right): \delta 203.5,138.3,137.8,137.3,131.9,131.6,128.8,128.3,127.6,125.6,73.3,73.2,67.2,44.5$, 21.3. HPLC (Chiralcel AS-H column, hexane $/{ }^{i} \mathrm{PrOH}=88 / 12,0.8 \mathrm{~mL} \mathrm{~min}^{-1}, 220 \mathrm{~nm}$ ): $\mathrm{t}_{1}=12.5 \mathrm{~min}, \mathrm{t}_{2}$ $=16.5$ min. HRMS Calculated for $\mathrm{C}_{18} \mathrm{H}_{20} \mathrm{O}_{3} \mathrm{Na}(\mathrm{M}+\mathrm{Na})^{+}:$307.1310, found: 307.1311.

\section{(2i) 4-(Benzyloxy)-3-hydroxy-1-(naphthalen-1-yl)butan-1-one}

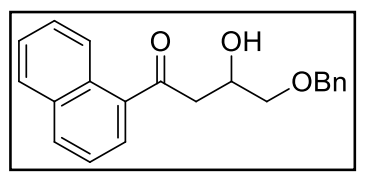

Purified by flash column chromatography $(\mathrm{PE} / \mathrm{EA}=5: 1)$ as light yellow oil (296 mg, 92\%). ${ }^{1} \mathbf{H}$ NMR (400 MHz, $\left.\mathrm{CDCl}_{3}\right): \delta 8.47$ (s, 1H), 8.06-7.84 (m, 4H), 7.66$7.52(\mathrm{~m}, 2 \mathrm{H}), 7.40-7.27(\mathrm{~m}, 5 \mathrm{H}), 4.61(\mathrm{~d}, J=1.8 \mathrm{~Hz}, 2 \mathrm{H}), 4.52(\mathrm{dd}, J=10.0,5.0$ $\mathrm{Hz}, 1 \mathrm{H}), 3.68-3.57(\mathrm{~m}, 2 \mathrm{H}), 3.38-3.33(\mathrm{~m}, 2 \mathrm{H}), 3.25(\mathrm{~d}, J=4.0 \mathrm{~Hz}, 1 \mathrm{H}) .{ }^{13} \mathbf{C}$ NMR $\left(100 \mathrm{MHz}, \mathrm{CDCl}_{3}\right)$ : $\delta 199.4,137.8,135.4,133.9,132.2,129.9,129.4,128.4,128.2,127.6,127.5,126.6,123.4,73.2,67.0$, 41.8. HPLC (Chiralcel AD-H column, hexane $/{ }^{i} \mathrm{PrOH}=88 / 12,0.8 \mathrm{~mL} \mathrm{~min}^{-1}, 220 \mathrm{~nm}$ ): $\mathrm{t}_{1}=26.1 \mathrm{~min}, \mathrm{t}_{2}$ $=28.0$ min. HRMS Calculated for $\mathrm{C}_{21} \mathrm{H}_{20} \mathrm{O}_{3} \mathrm{Na}(\mathrm{M}+\mathrm{Na})^{+}:$343.1310, found: 343.1301 .

\section{(2j) 4-(Benzyloxy)-3-hydroxy-1-(naphthalen-2-yl)butan-1-one}

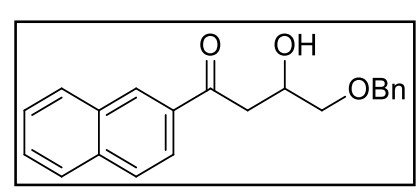

Purified by flash column chromatography $(\mathrm{PE} / \mathrm{EA}=5: 1)$ as light yellow oil (304 mg, 95\%). ${ }^{1} \mathbf{H}$ NMR (400 MHz, $\left.\mathrm{CDCl}_{3}\right): \delta 8.64(\mathrm{~d}, J=8.6 \mathrm{~Hz}, 1 \mathrm{H}), 8.02$ $7.87(\mathrm{~m}, 3 \mathrm{H}), 7.65-7.46(\mathrm{~m}, 3 \mathrm{H}), 7.41-7.25(\mathrm{~m}, 5 \mathrm{H}), 4.59$ (d, J=2.0 Hz, 2H), $4.57-4.48(\mathrm{~m}, 1 \mathrm{H}), 3.66-3.56(\mathrm{~m}, 2 \mathrm{H}), 3.38-3.24(\mathrm{~m}, 2 \mathrm{H}), 3.22(\mathrm{~d}, J=4.2 \mathrm{~Hz}, 1 \mathrm{H}) .{ }^{13} \mathbf{C} \mathbf{N M R}(100 \mathrm{MHz}$, $\left.\mathrm{CDCl}_{3}\right): \delta 203.4,137.7,135.2,133.6,132.8,129.8,128.2,128.0,127.8,127.5,126.2,125.5,124.1,73.2$, 73.1, 67.2, 45.0. HPLC (ChiralPak AS-H column, hexane $\left./{ }^{i} \mathrm{PrOH}=88 / 12,0.8 \mathrm{~mL} \mathrm{~min}{ }^{-1}, 220 \mathrm{~nm}\right): \mathrm{t}_{1}=$ $20.6 \mathrm{~min}, \mathrm{t}_{2}=22.2 \mathrm{~min}$. HRMS Calculated for $\mathrm{C}_{21} \mathrm{H}_{20} \mathrm{O}_{3} \mathrm{Na}(\mathrm{M}+\mathrm{Na})^{+}:$343.1310, found: 343.1299.

\section{(2k) 4-(Benzyloxy)-1-(furan-2-yl)-3-hydroxybutan-1-one}

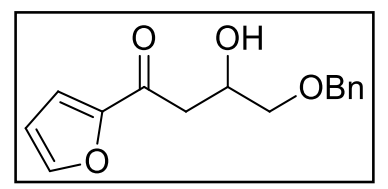

Purified by flash column chromatography $(\mathrm{PE} / \mathrm{EA}=5: 1)$ as light yellow liquid (245 mg, 94\%). Light yellow oil. ${ }^{1} \mathbf{H}$ NMR (400 MHz, $\left.\mathrm{CDCl}_{3}\right): \delta 7.60$ (d, $J=1.6$ $\mathrm{Hz}, 1 \mathrm{H}), 7.40-7.26(\mathrm{~m}, 5 \mathrm{H}), 7.22(\mathrm{~d}, J=3.6 \mathrm{~Hz}, 1 \mathrm{H}), 6.54(\mathrm{dd}, J=3.6,1.8 \mathrm{~Hz}$, $1 \mathrm{H}), 4.58(\mathrm{~d}, J=1.6 \mathrm{~Hz}, 2 \mathrm{H}), 4.42(\mathrm{~s}, 1 \mathrm{H}), 3.56(\mathrm{qd}, J=9.6,5.2 \mathrm{~Hz}, 2 \mathrm{H}), 3.13-3.00(\mathrm{~m}, 2 \mathrm{H}), 3.06$ (s, 1H). $\left.{ }^{13} \mathbf{C ~ N M R ~ ( 1 0 0 ~ M H z , ~} \mathrm{CDCl}_{3}\right): \delta 188.1,152.4,146.7,137.8,128.3,127.6,117.8,112.3,73.3,73.2$, 
66.9, 41.6. HPLC (Chiralcel AS-H column, hexane $\left./{ }^{i} \mathrm{PrOH}\left(1 \% \mathrm{Et}_{2} \mathrm{NH}\right)=86 / 14,1.0 \mathrm{~mL} \mathrm{~min}^{-1}, 220 \mathrm{~nm}\right)$ : $\mathrm{t}_{1}=18.6 \mathrm{~min}, \mathrm{t}_{2}=27.4 \mathrm{~min}$. HRMS Calculated for $\mathrm{C}_{15} \mathrm{H}_{16} \mathrm{O}_{4} \mathrm{Na}(\mathrm{M}+\mathrm{Na})^{+}:$283.0946, found: 283.0953 .

\section{Preparation of 3a-j}

(3a) 4,4-Diethoxy-1-phenylbutane-1,3-dione ${ }^{[10]}$

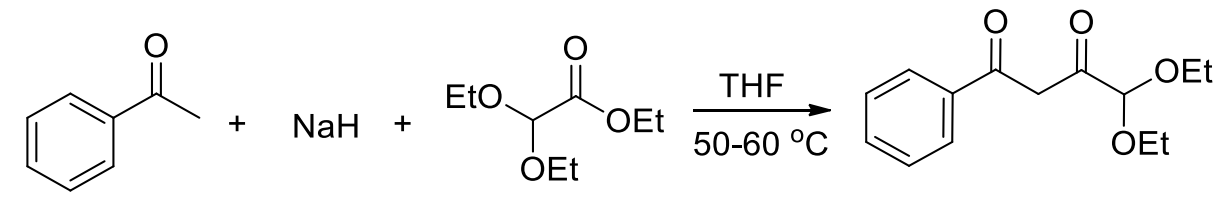

To a suspension of $60 \% \mathrm{NaH}(2.9 \mathrm{~g}, 73.2 \mathrm{mmol})$ in of THF $(60 \mathrm{~mL})$ was added a solution of acetophenone $(4.0 \mathrm{~g}, 33.3 \mathrm{mmol})$ and ethyl 2,2-diethoxyacetate $(5.9 \mathrm{~g}, 33.3 \mathrm{mmol})$ in $\mathrm{THF}(40 \mathrm{~mL})$ at $50{ }^{\circ} \mathrm{C}$ and stirred overnight. Then poured the mixture into $50 \mathrm{~mL}$ cold $10 \% \mathrm{HCl}$ (aq.) and the solvent was removed under reduced pressure. The aqueous phase was extracted with ethyl acetate for three times. The combined organic phase was washed with brine and dried over $\mathrm{Na}_{2} \mathrm{SO}_{4}$. Column chromatography (PE/EA = 10:1) gave 3a as light yellow liquid $(6.9 \mathrm{~g}, 84 \%)$. ${ }^{\mathbf{1}} \mathbf{H} \mathbf{N M R}\left(400 \mathrm{MHz}, \mathrm{CDCl}_{3}\right)$ : mixture of keto:enol = 5:95, $\delta$ 7.96-7.89 (m, 2H), 7.58-7.50 (m, 1H), 7.48-7.44 (m, 2H), 6.61-6.57 (m, 1H), 4.92-4.87 (m, 1H), 3.78$3.60(\mathrm{~m}, 4 \mathrm{H}), 1.28(\mathrm{t}, J=7.2 \mathrm{~Hz}, 6 \mathrm{H}) .{ }^{13} \mathbf{C ~ N M R}\left(100 \mathrm{MHz}, \mathrm{CDCl}_{3}\right): \delta 190.4,184.4,134.4,132.6,128.6$, $127.2,100.0,93.7,62.5,15.1$.

\section{(3b) 4,4-Diethoxy-1-(thiophen-2-yl)butane-1,3-dione}

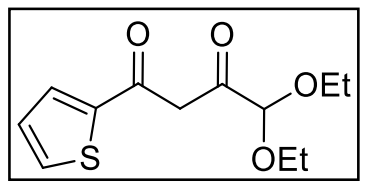

Prepared from acetylthiophene $(2.0 \mathrm{~g}, 15.8 \mathrm{mmol})$ and ethyl 2,2-diethoxyacetate $(2.8 \mathrm{~g}, 15.8 \mathrm{mmol})$ with the same procedure as 3a. Purified by flash column chromatography $(\mathrm{PE} / \mathrm{EA}=8: 1)$ gave the product as light brown liquid $(3.2 \mathrm{~g}$, 79\%). ${ }^{1} \mathbf{H}$ NMR (400 MHz, $\mathrm{CDCl}_{3}$ ): mixture of keto:enol = 17:83, $\delta 7.81-7.69(\mathrm{~m}, 1 \mathrm{H}), 7.68-7.63(\mathrm{~m}$, 1H), 7.15-7.12 (m, 1H), $6.43(\mathrm{~s}, 1 \mathrm{H}), 4.94$ and $4.16(\mathrm{~s}, 1 \mathrm{H}), 3.79-3.53(\mathrm{~m}, 4 \mathrm{H}), 1.30-1.20(\mathrm{~m}, 6 \mathrm{H}) .{ }^{13} \mathrm{C}$ NMR (100 MHz, $\left.\mathrm{CDCl}_{3}\right): \delta 183.2,182.6,141.2,134.5,133.1,130.91,128.2,128.1,102.1,99.0,94.2$, 63.5, 62.2, 48.5, 29.5, 15.0, 14.9. HRMS Calculated for $\mathrm{C}_{12} \mathrm{H}_{16} \mathrm{O}_{4} \mathrm{SNa}(\mathrm{M}+\mathrm{Na})^{+}:$279.0667, found: 279.0662 .

(3c) 2-(2,4-Dioxopentyl)isoindoline-1,3-dione ${ }^{[11]}$
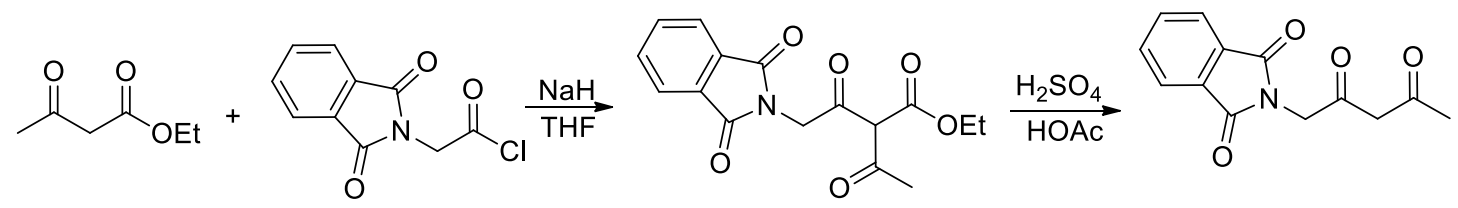

Ethyl 2-acetyl-4-(1,3-dioxoisoindolin-2-yl)-3-oxobutanoate: To a suspension of $60 \% \mathrm{NaH}(2.1 \mathrm{~g}$, $50.7 \mathrm{mmol})$ in THF $(50 \mathrm{~mL})$ was added ethyl acetoacetate $(6.0 \mathrm{~g}, 46.1 \mathrm{mmol})$ at room temperature. Then 
2-(1,3-dioxoisoindolin-2-yl)acetyl chloride $(10.9 \mathrm{~g}, 48.0 \mathrm{mmol})^{[12]}$ in $100 \mathrm{~mL}$ of THF was added dropwise at $40{ }^{\circ} \mathrm{C}$. After addtion, the mixture was reacted at $70{ }^{\circ} \mathrm{C}$ overnight. The mixture was poured into $100 \mathrm{~mL}$ cold $10 \% \mathrm{HCl}$ (aq.) and the solvent was removed under reduced pressure. The aqueous phase was extracted with ethyl acetate for three times. The combined organic phase was washed with saturated brine and dried over $\mathrm{Na}_{2} \mathrm{SO}_{4}$. The crude product was recrystallized from EtOH to give light yellow solid (11.3 g, 77\%). ${ }^{1} \mathbf{H}$ NMR $\left(400 \mathrm{MHz} \mathrm{CDCl}_{3}\right): \delta 7.89(\mathrm{dd}, J=5.6,3.0 \mathrm{~Hz}, 2 \mathrm{H}), 7.75(\mathrm{dd}, J=$ 5.4, 3.2 Hz, 2H), 4.99 (s, 2H), $4.34(\mathrm{~d}, J=7.2 \mathrm{~Hz}, 2 \mathrm{H}), 2.48(\mathrm{~s}, 3 \mathrm{H}), 1.39$ (t, $J=7.2 \mathrm{~Hz}, 3 \mathrm{H}) .{ }^{13} \mathbf{C}$ NMR $\left(100 \mathrm{MHz}, \mathrm{CDCl}_{3}\right): \delta 195.4,194.7,168.0,165.9,134.1,132.1,123.5,106.3,60.9,44.7,25.0,14.3$.

Ethyl 2-acetyl-4-(1,3-dioxoisoindolin-2-yl)-3-oxobutanoate (6.8 g, $22.1 \mathrm{mmol})$ was dissolved in acetic acid $(60 \mathrm{~mL})$ containing several drops of concentrated sulfuric acid. The mixture was stirred under reflux for $2 \mathrm{~h}$ and the acetic acid was removed under reduced pressure to afford a brown crude mixture. Column chromatography (PE/EA = 3:1) gave the products as white solid $(4.8 \mathrm{~g}, 88 \%) .{ }^{\mathbf{1}} \mathbf{H}$ NMR $(400$ $\left.\mathrm{MHz}, \mathrm{CDCl}_{3}\right)$ : mixture of keto:enol $=40: 60, \delta 7.90(\mathrm{dd}, J=5.4,3.0 \mathrm{~Hz}, 2 \mathrm{H}), 7.75(\mathrm{dd}, J=5.4,3.0 \mathrm{~Hz}$, 2H), $5.57(\mathrm{~s}, \mathrm{CH}=\mathrm{CO}), 4.60$ and $4.50(\mathrm{~s}, 2 \mathrm{H}), 3.71\left(\mathrm{~s}, \mathrm{COCH}_{2-}\right), 2.29$ and $2.04(\mathrm{~s}, 3 \mathrm{H}) .{ }^{13} \mathbf{C ~ N M R}(100$ $\left.\mathrm{MHz}, \mathrm{CDCl}_{3}\right): \delta 191.3,185.6,167.6,134.2,134.1,131.9,123.5,97.3,55.4,47.0,43.4,26.9,23.0$.

\section{(3d) 1-(Phenylsulfonyl)pentane-2,4-dione ${ }^{[13]}$}

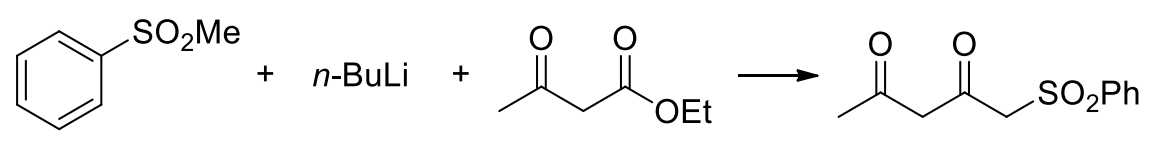

To a solution of (methylsulfonyl)benzene $(5.0 \mathrm{~g}, 32.0 \mathrm{mmol})$ in THF $(70 \mathrm{~mL})$ was added $n$-BuLi (30 mL, 2.4 $\mathrm{M}$ in hexane) under nitrogen at $0{ }^{\circ} \mathrm{C}$. After $1 \mathrm{~h}$, the mixture was cooled to $-10{ }^{\circ} \mathrm{C}$ and ethyl acetoacetate $(4.2 \mathrm{~g}, 32.0 \mathrm{mmol})$ was added dropwise and warmed to room temperature. After stirring overnight, the reaction was neutralized with cold $10 \% \mathrm{HCl}$ aq. Then THF was removed under reduced pressure. The aqueous phase was extracted with ethyl acetate $(30 \mathrm{~mL} \times 3)$. Combined organic phase was washed with saturated brine, dried over $\mathrm{Na}_{2} \mathrm{SO}_{4}$. The crude product was purified by column chromatography (PE/EA = 3:1) to give white solid (5.7 g, 74\%). m.p.: 105.3-106.1 ${ }^{\circ} \mathrm{C} .{ }^{1} \mathbf{H}$ NMR (400 $\left.\mathrm{MHz}, \mathrm{CDCl}_{3}\right)$ : mixture of keto:enol = 13:87, $\delta 7.92-7.86(\mathrm{~m}, 2 \mathrm{H}), 7.67(\mathrm{~d}, J=7.6 \mathrm{~Hz}, 1 \mathrm{H}), 7.60-7.54$ $(\mathrm{m}, 2 \mathrm{H}), 5.73$ (s, $\mathrm{CH}=\mathrm{CO}), 4.01\left(\mathrm{~s}, \mathrm{PhSO}_{2} \mathrm{CH}_{2-}, 2 \mathrm{H}\right), 2.26$ and $2.10\left(\mathrm{~s},-\mathrm{COCH}_{3}, 3 \mathrm{H}\right) .{ }^{13} \mathbf{C ~ N M R}(100$ $\left.\mathrm{MHz}, \mathrm{CDCl}_{3}\right): \delta 192.4,179.5,138.4,134.1,129.1,128.1,102.5,64.1,57.5,24.7$.

\section{(3e) 1-Phenyl-4-(phenylsulfonyl)butane-1,3-dione}

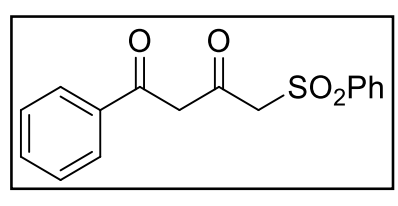

3e was prepared similarly as $\mathbf{3 d}$. White solid, m.p.: $120.6-122.5^{\circ} \mathrm{C} .{ }^{\mathbf{1}} \mathbf{H}$ NMR $\left(400 \mathrm{MHz}, \mathrm{CDCl}_{3}\right)$ : mixture of keto:enol = 4:96, $\delta 7.94-7.84(\mathrm{~m}, 4 \mathrm{H}), 7.74$ $7.61(\mathrm{~m}, 1 \mathrm{H}), 7.57(\mathrm{t}, J=7.6 \mathrm{~Hz}, 3 \mathrm{H}), 7.47(\mathrm{t}, J=7.6 \mathrm{~Hz}, 2 \mathrm{H}), 6.40(\mathrm{~s}, 1 \mathrm{H})$, 
4.16 (s, 2H). ${ }^{13} \mathrm{C}$ NMR (100 MHz, $\left.\mathrm{CDCl}_{3}\right): \delta 184.3,181.8,138.4,134.2,133.6,133.1,129.2,128.7$, 128.3, 127.3, 98.6, 65.0. HRMS Calculated for $\mathrm{C}_{16} \mathrm{H}_{15} \mathrm{O}_{4} \mathrm{~S}(\mathrm{M}+\mathrm{H})^{+}:$: 303.0691, found: 303.0690 .

\section{(3f) 4,4,4-Trifluoro-1-phenylbutane-1,3-dione ${ }^{[14]}$}

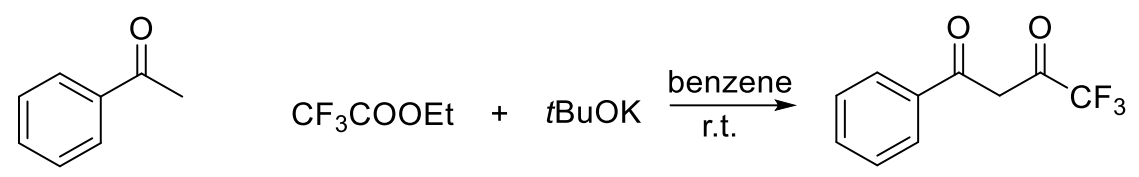

To a suspension of $t$-BuOK $(1.4 \mathrm{~g}, 12.0 \mathrm{mmol})$ and acetophenone $(1.2 \mathrm{~g}, 10.0 \mathrm{mmol})$ in benzene $(10$ $\mathrm{mL}$ ) was added ethyl trifluoacetate $(1.7 \mathrm{~g}, 12.0 \mathrm{mmol})$ dropwise at $10{ }^{\circ} \mathrm{C}$. The reaction mixture was stirred at room temperature for $15 \mathrm{~h}$ and acidified by $10 \%$ aq. $\mathrm{H}_{2} \mathrm{SO}_{4}$ to $\mathrm{pH}=7$ before extracted with ethyl ether $(20 \mathrm{~mL} \times 3)$. The combined organic phase was washed with brine and dried over $\mathrm{Na}_{2} \mathrm{SO}_{4}$. The crude product was purified by column chromatography $(\mathrm{PE} / \mathrm{EA}=10: 1)$ to give $\mathbf{3 f}$ as light yellow oil (1.5 g, 69\%). ${ }^{1} \mathbf{H}$ NMR (400 MHz, $\left.\mathrm{CDCl}_{3}\right)$ : mixture of keto:enol = 4:96, $\delta$ 7.94-7.82 (m, 2H), 7.56 (ddd, $J=6.8,4.0,1.2 \mathrm{~Hz}, 1 \mathrm{H}), 7.45-7.39(\mathrm{~m}, 2 \mathrm{H}), 6.51(\mathrm{~s}, 1 \mathrm{H}) .{ }^{13} \mathbf{C ~ N M R}\left(100 \mathrm{MHz}, \mathrm{CDCl}_{3}\right): \delta 186.2,177.2$ $\left(\mathrm{q}, J_{\mathrm{F}-\mathrm{C}}=36.6 \mathrm{~Hz}\right), 134.0,132.7,128.9,128.4,127.5,118.6,115.7,92.2,38.0$.

\section{(3g) 1-(2-Chlorophenyl)-3-phenylpropane-1,3-dione ${ }^{[15]}$}

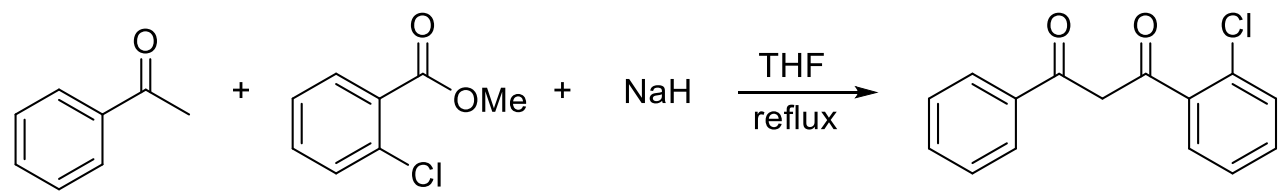

$\mathbf{3 g}$ was synthesized from acetophenone $(1.8 \mathrm{~g}, 15.0 \mathrm{mmol})$ and methyl $o$-chlorobenzoate $(2.6 \mathrm{~g}, 15.0$ $\mathrm{mmol})$ by using $\mathrm{NaH}(60 \%$ dispersed in mineral oil, $1.5 \mathrm{~g}, 37.5 \mathrm{mmol}$ ) according to the reported procedure. ${ }^{[15]}$ The crude product was purified by column chromatography (PE/EA $\left.=15: 1\right)$ to give yellow solid 3g (3.3 g, 84\%). ${ }^{1} \mathbf{H}$ NMR (400 MHz, $\left.\mathrm{CDCl}_{3}\right): \delta 8.09-7.78(\mathrm{~m}, 2 \mathrm{H}), 7.71-7.67(\mathrm{~m}, 1 \mathrm{H}), 7.59-7.27$ $(\mathrm{m}, 6 \mathrm{H}), 6.76-6.66(\mathrm{~m}, 1 \mathrm{H}) .{ }^{13} \mathrm{C}$ NMR $\left(100 \mathrm{MHz}, \mathrm{CDCl}_{3}\right): \delta 186.8,184.4,136.1,134.8,132.6,131.6$, 130.6, 130.0, 128.6, 127.2, 126.9, 98.3.

\section{(3h) Ethyl 2,4-dioxo-4-phenylbutanoate ${ }^{[16]}$}<smiles>CCOC(=O)C(=O)OCC</smiles>

To a solution of acetophenone $(2.4 \mathrm{~g}, 20.0 \mathrm{mmol})$ in toluene $(40 \mathrm{~mL})$ was added $\mathrm{NaH}(60 \%$ in mineral oil, $1.0 \mathrm{~g}, 42.0 \mathrm{mmol}$ ) in portions and the mixture was warmed to $45-50{ }^{\circ} \mathrm{C}$ under stirring. At this temperature a solution of diethyloxalate $(3.5 \mathrm{~g}, 24.0 \mathrm{mmol})$ in toluene $(20 \mathrm{~mL})$ was added dropwise. The reaction mixture was refluxed for another $1.5 \mathrm{~h}$. Then cooled to room temperature and acidified by $10 \%$ 
aq. $\mathrm{H}_{2} \mathrm{SO}_{4}$ to $\mathrm{pH}=6$ before extracted with ethyl acetate $(20 \mathrm{~mL} \times 3)$. The combined organic phase was washed with brine and dried over $\mathrm{Na}_{2} \mathrm{SO}_{4}$. The crude product was purified by column chromatography $(\mathrm{PE} / \mathrm{EA}=4: 1)$ to give $\mathbf{3 h}$ as light yellow liquid $(4.1 \mathrm{~g}, 93 \%) .{ }^{\mathbf{1}} \mathbf{H} \mathbf{~ N M R}\left(400 \mathrm{MHz}, \mathrm{CDCl}_{3}\right): \delta 8.08-7.90$ $(\mathrm{m}, 2 \mathrm{H}), 7.61(\mathrm{t}, J=7.4 \mathrm{~Hz}, 1 \mathrm{H}), 7.51(\mathrm{t}, J=7.6 \mathrm{~Hz}, 2 \mathrm{H}), 7.08(\mathrm{~s}, 1 \mathrm{H}), 4.40(\mathrm{q}, J=7.0 \mathrm{~Hz}, 2 \mathrm{H}), 1.41(\mathrm{t}$, $J=7.0 \mathrm{~Hz}, 3 \mathrm{H}) .{ }^{13} \mathrm{C}$ NMR $\left(100 \mathrm{MHz}, \mathrm{CDCl}_{3}\right): \delta 190.6,169.6,162.0,134.7,133.76,128.8,127.7,97.8$, $62.5,14.0$.

\section{(3i) Ethyl 2,4-dioxo-5-phenylpentanoate ${ }^{[17]}$}<smiles>CCOC(=O)C(=O)ON=Nc1ccccc1</smiles>

To a stirred solution of $\mathrm{NaOEt}$ (prepared from of $1.2 \mathrm{~g}$ of sodium in $25 \mathrm{~mL}$ of absolute $\mathrm{EtOH}$ ) was added a mixture of 1-phenylpropan-2-one $(5.4 \mathrm{~g}, 40.3 \mathrm{mmol})$ and diethyl oxalate $(5.9 \mathrm{~g}, 40.3 \mathrm{mmol})$ at $0{ }^{\circ} \mathrm{C}$. After $5 \mathrm{~h}$ at room temperature, the mixture was acidified by $10 \%$ aq. $\mathrm{H}_{2} \mathrm{SO}_{4}$ to $\mathrm{pH}=6$. Ethanol was removed under reduced pressure and the residue was extracted with ethyl acetate $(20 \mathrm{~mL} \times 3)$. The combined organic phase was washed with saturated brine and dried over $\mathrm{Na}_{2} \mathrm{SO}_{4}$. The crude product was purified by column chromatography $(\mathrm{PE} / \mathrm{EA}=3: 1)$ to give $\mathbf{3 i}$ as yellow oil $(7.6 \mathrm{~g}, 81 \%) .{ }^{1} \mathbf{H} \mathbf{~ N M R}(400$ $\left.\mathrm{MHz}, \mathrm{CDCl}_{3}\right)$ : mixture of keto:enol = 13:87, $\delta 7.39-7.19(\mathrm{~m}, 5 \mathrm{H}), 6.36(\mathrm{~s},-\mathrm{CH}=\mathrm{CO}, 1 \mathrm{H}), 4.33-4.29(\mathrm{~m}$, 2H), 3.77 (s, 2H), 1.38-1.30 (m, 3H). $\left.{ }^{13} \mathbf{C ~ N M R ~ ( 1 0 0 ~ M H z , ~} \mathrm{CDCl}_{3}\right): \delta 200.5,166.4,161.6,133.4,129.2$, 128.7, 127.2, 101.4, 62.3, 47.4, 13.8 .

\section{(3j) 5,5-Dimethyl-1-morpholinohexane-1,2,4-trione ${ }^{[18]}$}

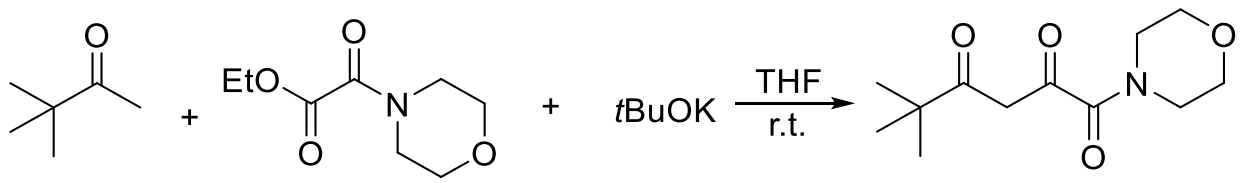

A suspension of $t$-BuOK $(2.3 \mathrm{~g}, 20.5 \mathrm{mmol})$ in THF $(20 \mathrm{~mL})$ was added via cannula to a solution of pinacolone (1.0 g, $10.0 \mathrm{mmol})$ and ethyl 2-morpholino-2-oxoacetate $(1.9 \mathrm{~g}, 10.0 \mathrm{mmol})$ in THF (40 mL) over $40 \mathrm{~min}$ at $25^{\circ} \mathrm{C}$. After $3 \mathrm{~h}, \mathrm{AcOH}(2.1 \mathrm{~mL}, 36.0 \mathrm{mmol})$ was added over $5 \mathrm{~min}$ and the resulting heterogeneous mixture was filtered and the solids were washed with $\mathrm{CH}_{2} \mathrm{Cl}_{2}$. The filtrate was washed with $\mathrm{NaHCO}_{3}$ and brine, dried, filtered, and concentrated. Purification of the residue by flash chromatography $(\mathrm{PE} / \mathrm{EA}=1: 1)$ provided the title compound as a yellow solid $(1.8 \mathrm{~g}, 75 \%) .{ }^{\mathbf{1}} \mathbf{H}$ NMR $\left(400 \mathrm{MHz}, \mathrm{CDCl}_{3}\right)$ : mixture of keto:enol = 31:69, $\delta 6.03(\mathrm{~s}, 1 \mathrm{H}), 4.05(\mathrm{~s}, 1 \mathrm{H}), 3.78-3.57(\mathrm{~m}, 8 \mathrm{H}), 1.19$ $(\mathrm{s}, 9 \mathrm{H}) .{ }^{13} \mathrm{C}$ NMR $\left(100 \mathrm{MHz}, \mathrm{CDCl}_{3}\right): \delta 201.0,184.6,163.7,95.4,66.8,66.6,66.4,66.3,47.9,46.5,46.2$, 42.1, 38.9, 27.0, 26.6, 25.8. 


\section{Characterization data of compounds $4 a-j$}

\section{(4a) 4,4-Diethoxy-3-hydroxy-1-phenylbutan-1-one}

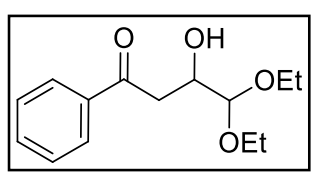

Purified by flash column chromatography $(\mathrm{PE} / \mathrm{EA}=6: 1)$ as light yellow oil $(225$ mg, 89\%). ${ }^{1} \mathbf{H}$ NMR (400 MHz, $\left.\mathrm{CDCl}_{3}\right): \delta 8.01-7.94(\mathrm{~m}, 2 \mathrm{H}), 7.60-7.52(\mathrm{~m}, 1 \mathrm{H})$, $7.48-7.44(\mathrm{~m}, 2 \mathrm{H}), 4.52(\mathrm{~d}, J=4.8 \mathrm{~Hz}, 1 \mathrm{H}), 4.29(\mathrm{~s}, 1 \mathrm{H}), 3.80-3.75(\mathrm{~m}, 2 \mathrm{H}), 3.64$ $3.58(\mathrm{~m}, 2 \mathrm{H}), 1.24-1.20(\mathrm{~m}, 6 \mathrm{H}) .{ }^{13} \mathbf{C} \mathbf{N M R}\left(100 \mathrm{MHz}, \mathrm{CDCl}_{3}\right): \delta 197.4,173.7,136.3,133.4,128.5$, 128.0, 67.0, 61.6, 42.1, 14.0. HPLC (Chiralcel AD-H column, hexane/ ${ }^{i} \mathrm{PrOH}=90 / 10,0.8 \mathrm{~mL} \mathrm{~min}^{-1}, 220$ $\mathrm{nm}): \mathrm{t}_{1}=12.4 \mathrm{~min}, \mathrm{t}_{2}=13.6 \mathrm{~min}$. HRMS Calculated for $\mathrm{C}_{14} \mathrm{H}_{20} \mathrm{O}_{4} \mathrm{Na}(\mathrm{M}+\mathrm{Na})^{+}: 275.1259$, found: 275.1247.

(4b) 4,4-Diethoxy-3-hydroxy-1-(thiophen-2-yl)butan-1-one

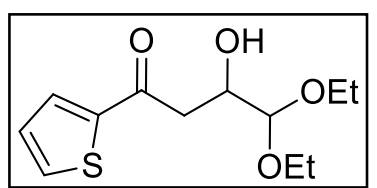

Purified by flash column chromatography $(\mathrm{PE} / \mathrm{EA}=3: 1)$ as yellow oil $(186 \mathrm{mg}$, 93\%). ${ }^{1} \mathbf{H}$ NMR (400 MHz, $\left.\mathrm{CDCl}_{3}\right): \delta 7.76(\mathrm{dd}, J=3.8,1.1 \mathrm{~Hz}, 1 \mathrm{H}), 7.65$ (dd, $J$ $=4.9,1.1 \mathrm{~Hz}, 1 \mathrm{H}), 7.13(\mathrm{dd}, J=4.9,3.8 \mathrm{~Hz}, 1 \mathrm{H}), 4.51(\mathrm{~d}, J=4.8 \mathrm{~Hz}, 1 \mathrm{H}), 4.27$ (d, $J=4.2 \mathrm{~Hz}, 1 \mathrm{H}), 3.80-3.75(\mathrm{~m}, 2 \mathrm{H}), 3.64-3.58(\mathrm{~m}, 2 \mathrm{H}), 3.26-3.11(\mathrm{~m}, 2 \mathrm{H}), 2.94(\mathrm{~d}, J=4.4 \mathrm{~Hz}, 1 \mathrm{H})$, 1.25-1.20 (m, 6H). ${ }^{13} \mathrm{C}$ NMR (100 MHz, $\left.\mathrm{CDCl}_{3}\right): \delta 192.6,144.3,134.0,132.6,128.1,103.8,69.1,64.0$, 63.6, 40.5, 15.3, 15.3. HPLC (ChiralPak AS-H column, hexane $/{ }^{i} \mathrm{PrOH}=92 / 8,0.7 \mathrm{~mL} \mathrm{~min}^{-1}, 254 \mathrm{~nm}$ ): $\mathrm{t}_{1}=15.7 \mathrm{~min}, \mathrm{t}_{2}=16.9$ min. HRMS Calculated for $\mathrm{C}_{12} \mathrm{H}_{18} \mathrm{O}_{4} \mathrm{SNa}(\mathrm{M}+\mathrm{Na})^{+}:$281.0832, found: 281.0829 .

\section{(4c) 2-(2-Hydroxy-4-oxopentyl)isoindoline-1,3-dione}

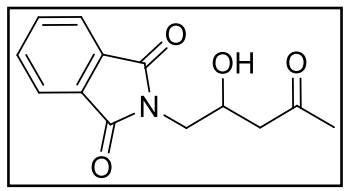

Purified by flash column chromatography $(\mathrm{PE} / \mathrm{EA}=2: 1)$ as white solid $(223 \mathrm{mg}$, 90\%), m.p.:112.1-113.3 ${ }^{\circ} \mathrm{C} .{ }^{1} \mathbf{H}$ NMR $\left(400 \mathrm{MHz}, \mathrm{CDCl}_{3}\right): \delta 7.86(\mathrm{dd}, J=5.5,3.0$ $\mathrm{Hz}, 2 \mathrm{H}), 7.73(\mathrm{dd}, J=5.4,3.0 \mathrm{~Hz}, 2 \mathrm{H}), 4.39-4.35(\mathrm{~m}, 1 \mathrm{H}), 3.88-3.72(\mathrm{~m}, 2 \mathrm{H})$, $3.23(\mathrm{~d}, J=4.4 \mathrm{~Hz}, 1 \mathrm{H}), 2.67(\mathrm{dd}, J=10.0,6.0 \mathrm{~Hz}, 2 \mathrm{H}), 2.19(\mathrm{~s}, 3 \mathrm{H}) .{ }^{13} \mathbf{C} \mathbf{N M R}\left(100 \mathrm{MHz}, \mathrm{CDCl}_{3}\right): \delta$ 208.2, 168.5, 134.0, 131.7, 123.3, 66.0, 47.3, 42.7, 30.6. HPLC (Chiralcel AD-H column, hexane/ ${ }^{i} \mathrm{PrOH}$ $\left.=82 / 18,0.75 \mathrm{~mL} \mathrm{~min}^{-1}, 220 \mathrm{~nm}\right): \mathrm{t}_{1}=25.2 \mathrm{~min}, \mathrm{t}_{2}=29.8 \mathrm{~min} . \mathbf{H R M S}$ Calculated for $\mathrm{C}_{13} \mathrm{H}_{13} \mathrm{NO}_{4} \mathrm{Na}$ $(\mathrm{M}+\mathrm{Na})^{+}: 270.0742$, found: 270.0730 .

(4d) 4-Hydroxy-5-(phenylsulfonyl)pentan-2-one

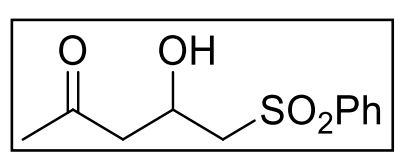

Purified by flash column chromatography (PE/EA = 3:1) as yellow oil (221 mg, 91\%). ${ }^{1} \mathbf{H}$ NMR (400 MHz, $\left.\mathrm{CDCl}_{3}\right): \delta 7.93-7.86(\mathrm{~m}, 2 \mathrm{H}), 7.68-7.61(\mathrm{~m}, 1 \mathrm{H})$, 7.58-7.52 (m, 2H), 4.54-4.41 (m, 1H), $3.61(\mathrm{~s}, 1 \mathrm{H}), 3.37-3.22(\mathrm{~m}, 2 \mathrm{H}), 2.75(\mathrm{~d}$, 
$J=5.6 \mathrm{~Hz}, 2 \mathrm{H}), 2.13(\mathrm{~s}, 3 \mathrm{H}) .{ }^{13} \mathbf{C} \mathbf{N M R}\left(100 \mathrm{MHz}, \mathrm{CDCl}_{3}\right): \delta 207.4,139.2,133.9,129.3,127.8,62.7$, 60.9, 48.5, 30.6. HPLC (ChiralPak IA-3 column, hexane $/{ }^{i} \mathrm{PrOH}=82 / 18,0.8 \mathrm{~mL} \mathrm{~min}^{-1}, 220 \mathrm{~nm}$ ): $\mathrm{t}_{1}=$ 28.6 min, $\mathrm{t}_{2}=30.8 \mathrm{~min}$. HRMS Calculated for $\mathrm{C}_{13} \mathrm{H}_{17} \mathrm{O}_{4} \mathrm{~S}(\mathrm{M}+\mathrm{H})^{+}: 243.0691$, found: 243.0690 .

\section{(4e) 3-Hydroxy-1-phenyl-4-(phenylsulfonyl)butan-1-one}

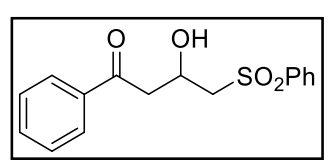

Purified by flash column chromatography $(\mathrm{PE} / \mathrm{EA}=3: 1)$ as white solid $(280 \mathrm{mg}$, 92\%), m.p.: 100.4-101.5 ${ }^{\circ} \mathrm{C} .{ }^{\mathbf{1}} \mathbf{H}$ NMR (400 MHz, $\left.\mathrm{CDCl}_{3}\right): \delta 8.00-7.89$ (m, 4H), $7.67(\mathrm{~d}, J=7.6 \mathrm{~Hz}, 1 \mathrm{H}), 7.60(\mathrm{t}, J=7.8 \mathrm{~Hz}, 3 \mathrm{H}), 7.47(\mathrm{t}, J=7.8 \mathrm{~Hz}, 2 \mathrm{H}), 4.71-4.67$ $(\mathrm{m}, 1 \mathrm{H}), 3.74(\mathrm{~d}, J=3.6 \mathrm{~Hz}, 1 \mathrm{H}), 3.46(\mathrm{dd}, J=6.0,1.8 \mathrm{~Hz}, 2 \mathrm{H}), 3.34(\mathrm{~d}, J=5.8 \mathrm{~Hz}, 2 \mathrm{H}) .{ }^{13} \mathbf{C} \mathbf{N M R}(100$ $\left.\mathrm{MHz}, \mathrm{CDCl}_{3}\right): \delta 198.6,139.3,136.2,134.0,133.8,129.3,128.7,128.1,128.0,63.2,61.2$, 43.9. HPLC (ChiralPak IA-3 column, hexane $/{ }^{i} \mathrm{PrOH}=81 / 19,0.9 \mathrm{~mL} \mathrm{~min}{ }^{-1}, 254 \mathrm{~nm}$ ): $\mathrm{t}_{1}=26.4 \mathrm{~min}, \mathrm{t}_{2}=28.5 \mathrm{~min}$. HRMS Calculated for $\mathrm{C}_{16} \mathrm{H}_{16} \mathrm{O}_{4} \mathrm{SNa}(\mathrm{M}+\mathrm{Na})^{+}: 327.0667$, found: 327.0657 .

\section{(4f) $(R)-4,4,4-$ Trifluoro-3-hydroxy-1-phenylbutan-1-one ${ }^{[19]}$}

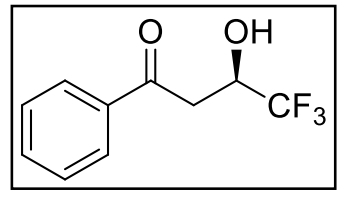

Purified by flash column chromatography $(\mathrm{PE} / \mathrm{EA}=8: 1)$ as colorless liquid (208 mg, 95\%). ${ }^{1} \mathbf{H}$ NMR (400 MHz, $\left.\mathrm{CDCl}_{3}\right): \delta 7.97(\mathrm{dd}, J=8.2,1.0 \mathrm{~Hz}, 2 \mathrm{H}), 7.62(\mathrm{~d}$, $J=7.8 \mathrm{~Hz}, 1 \mathrm{H}), 7.51(\mathrm{t}, J=7.8 \mathrm{~Hz}, 2 \mathrm{H}), 4.69(\mathrm{~s}, 1 \mathrm{H}), 3.51(\mathrm{~d}, J=4.6 \mathrm{~Hz}, 1 \mathrm{H})$, 3.43-3.27 (m, 2H). ${ }^{13} \mathbf{C}$ NMR (100 MHz, $\left.\mathrm{CDCl}_{3}\right): \delta 197.5,136.9,134.1,128.8,128.2,124.69$ (q, $J=$ 280.0 Hz), 67.03 (q, $J=32.0 \mathrm{~Hz}$ ), 38.3. HPLC (Chiralcel OD-H column, hexane $/{ }^{i} \mathrm{PrOH}=95 / 5,1.0 \mathrm{~mL}$ $\left.\min ^{-1}, 237 \mathrm{~nm}\right): \mathrm{t}_{1}=9.5 \mathrm{~min}, \mathrm{t}_{2}=10.7 \mathrm{~min}$.

(4g) (R)-3-(2-Chlorophenyl)-3-hydroxy-1-phenylpropan-1-one ${ }^{[20]}$

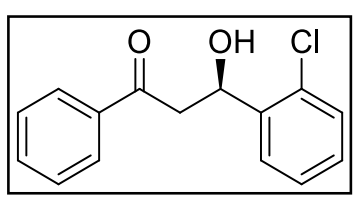

Purified by flash column chromatography $(\mathrm{PE} / \mathrm{EA}=5: 1)$ as yellowoil $(242 \mathrm{mg}$, 93\%). ${ }^{1} \mathbf{H}$ NMR (400 MHz, $\left.\mathrm{CDCl}_{3}\right): \delta 8.00-7.93(\mathrm{~m}, 2 \mathrm{H}), 7.72(\mathrm{~d}, J=7.8 \mathrm{~Hz}, 1 \mathrm{H})$, $7.59(\mathrm{dd}, J=10.7,4.2 \mathrm{~Hz}, 1 \mathrm{H}), 7.47(\mathrm{t}, J=7.7 \mathrm{~Hz}, 2 \mathrm{H}), 7.35(\mathrm{t}, J=8.0 \mathrm{~Hz}, 2 \mathrm{H})$, 7.29-7.20 (m, 2H), 5.69 (d, $J=9.6 \mathrm{~Hz}, 1 \mathrm{H}), 3.84(\mathrm{~d}, J=3.2 \mathrm{~Hz}, 1 \mathrm{H}), 3.60-3.55(\mathrm{~m}, 1 \mathrm{H}), 3.18-3.12(\mathrm{~m}$, 1H). ${ }^{13} \mathrm{C} \mathrm{NMR}\left(100 \mathrm{MHz}, \mathrm{CDCl}_{3}\right): \delta 200.1,140.3,136.3,133.6,131.0,129.2,128.6,128.5,128.1,127.2$,

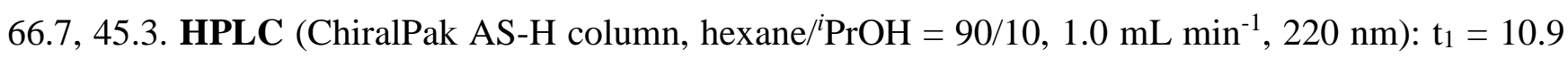
$\min , \mathrm{t}_{2}=14.4 \mathrm{~min}$. 


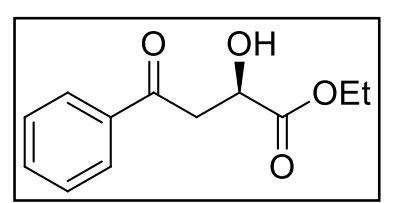

Purified by flash column chromatography $(\mathrm{PE} / \mathrm{EA}=6: 1)$ as yellowoil $(207 \mathrm{mg}$, 93\%). ${ }^{1} \mathbf{H}$ NMR (400 MHz, $\left.\mathrm{CDCl}_{3}\right): \delta 8.00-7.90(\mathrm{~m}, 2 \mathrm{H}), 7.63-7.53(\mathrm{~m}, 1 \mathrm{H})$,

$7.52-7.42(\mathrm{~m}, 2 \mathrm{H}), 4.66(\mathrm{~d}, J=3.8 \mathrm{~Hz}, 1 \mathrm{H}), 4.27(\mathrm{q}, J=7.2 \mathrm{~Hz}, 2 \mathrm{H}), 3.57-3.43$ $(\mathrm{m}, 2 \mathrm{H}), 3.35(\mathrm{~d}, J=5.8 \mathrm{~Hz}, 1 \mathrm{H}), 1.28(\mathrm{t}, J=7.2 \mathrm{~Hz}, 3 \mathrm{H}) .{ }^{13} \mathbf{C}$ NMR $\left(100 \mathrm{MHz}, \mathrm{CDCl}_{3}\right): \delta 197.4,173.7$, 136.3, 133.4, 128.5, 128.0, 67.0, 61.6, 42.1, 14.0. HPLC (ChiralPak AS-H column, hexane $/{ }^{i} \mathrm{PrOH}=$ $\left.85 / 15,1.0 \mathrm{~mL} \mathrm{~min}{ }^{-1}, 254 \mathrm{~nm}\right): \mathrm{t}_{1}=13.5 \mathrm{~min}, \mathrm{t}_{2}=16.7 \mathrm{~min}$.

\section{(4i) Ethyl 2-hydroxy-4-oxo-5-phenylpentanoate}

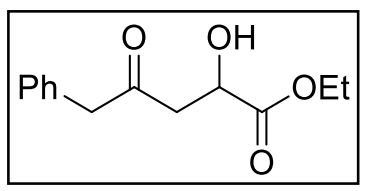

Purified by flash column chromatography $(\mathrm{PE} / \mathrm{EA}=5: 1)$ as yellow oil $(219 \mathrm{mg}$, 86\%). ${ }^{1} \mathbf{H}$ NMR $\left(400 \mathrm{MHz}, \mathrm{CDCl}_{3}\right) \delta 7.39-7.13(\mathrm{~m}, 5 \mathrm{H}), 4.44(\mathrm{~s}, 1 \mathrm{H}), 4.20$ (q, $J$ $=7.2 \mathrm{~Hz}, 2 \mathrm{H}), 3.73(\mathrm{~s}, 2 \mathrm{H}), 3.18(\mathrm{~d}, J=5.4 \mathrm{~Hz}, 1 \mathrm{H}), 2.95(\mathrm{dd}, J=9.0,5.0 \mathrm{~Hz}$, 2H), $1.24(\mathrm{t}, J=7.2 \mathrm{~Hz}, 3 \mathrm{H}) .{ }^{13} \mathrm{C}$ NMR $\left(100 \mathrm{MHz}, \mathrm{CDCl}_{3}\right): \delta 205.6,173.4,133.2,129.3,128.5,126.9$, 66.7, 61.6, 50.2, 45.0, 13.8. HPLC (ChiralCel AD-H column, hexane $/ i \mathrm{PrOH}=85 / 15,1.0 \mathrm{~mL} \mathrm{~min}^{-1}, 220$ $\mathrm{nm}): \mathrm{t}_{1}=11.3 \mathrm{~min}, \mathrm{t}_{2}=12.4$ min. HRMS Calculated for $\mathrm{C}_{13} \mathrm{H}_{16} \mathrm{O}_{4} \mathrm{Na}(\mathrm{M}+\mathrm{Na})^{+}:$259.0946, found: 259.0955 .

\section{(4j) 2-Hydroxy-5,5-dimethyl-1-morpholinohexane-1,4-dione ${ }^{[22]}$}

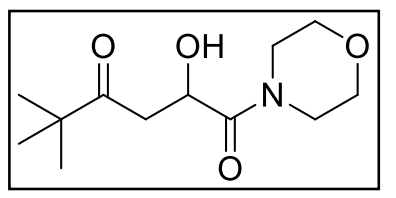

Purified by flash column chromatography $(\mathrm{PE} / \mathrm{EA}=5: 1)$ as yellow liquid (224 mg, 92\%). ${ }^{1} \mathbf{H}$ NMR (400 MHz, $\left.\mathrm{CDCl}_{3}\right): \delta 4.83(\mathrm{td}, J=7.6,3.2 \mathrm{~Hz}, 1 \mathrm{H}), 3.86$ $(\mathrm{d}, J=7.4 \mathrm{~Hz}, 1 \mathrm{H}), 3.80-3.45(\mathrm{~m}, 8 \mathrm{H}), 3.01-3.95(\mathrm{~m}, 1 \mathrm{H}), 2.58-2.53(\mathrm{~m}, 1 \mathrm{H})$, $1.16(\mathrm{~s}, 9 \mathrm{H}) .{ }^{13} \mathrm{C}$ NMR $\left(100 \mathrm{MHz}, \mathrm{CDCl}_{3}\right): \delta 213.8,171.5,66.4,66.3,65.0,45.4,44.1,42.4,41.0,25.7$. HPLC (ChiralPak AS-H column, hexane $/{ }^{i} \mathrm{PrOH}=80 / 20,0.8 \mathrm{~mL} \mathrm{~min}^{-1}, 220 \mathrm{~nm}$ ): $\mathrm{t}_{1}=8.8 \mathrm{~min}, \mathrm{t}_{2}=9.5$ min. HRMS Calculated for $\mathrm{C}_{12} \mathrm{H}_{21} \mathrm{NO}_{4} \mathrm{Na}(\mathrm{M}+\mathrm{Na})^{+}:$266.1368, found: 266.1370 .

\section{Study of the enol structure of $1 \mathrm{a}$ in THF.}

The following ${ }^{1} \mathrm{H}$ NMR and ${ }^{1} \mathrm{H}^{-13} \mathrm{C}$ HMBC spectra were recorded on a Brucker-500 $\mathrm{MHz} \mathrm{NMR}$ spectrometer at room temperature. The enol content in $d_{8}$-THF is $87 \%$ from the integrals of the $\mathrm{H}_{(1)}$. The tautomerization equilibrium constant $K_{e q}$ is calculated to be $6.7 .{ }^{[23]}$ An asymmetric $\beta$-diketone 1a may exist in two possible enol forms (B and $\mathbf{C}$ ), which could be identified by an HMBC experiment. ${ }^{\text {[24] }}$ 


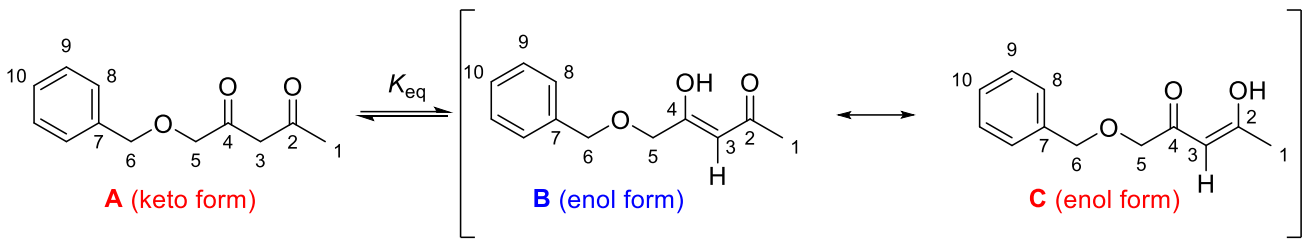

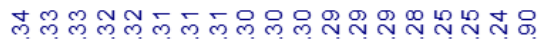

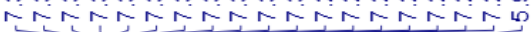

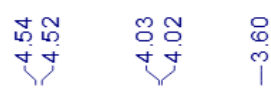

둥
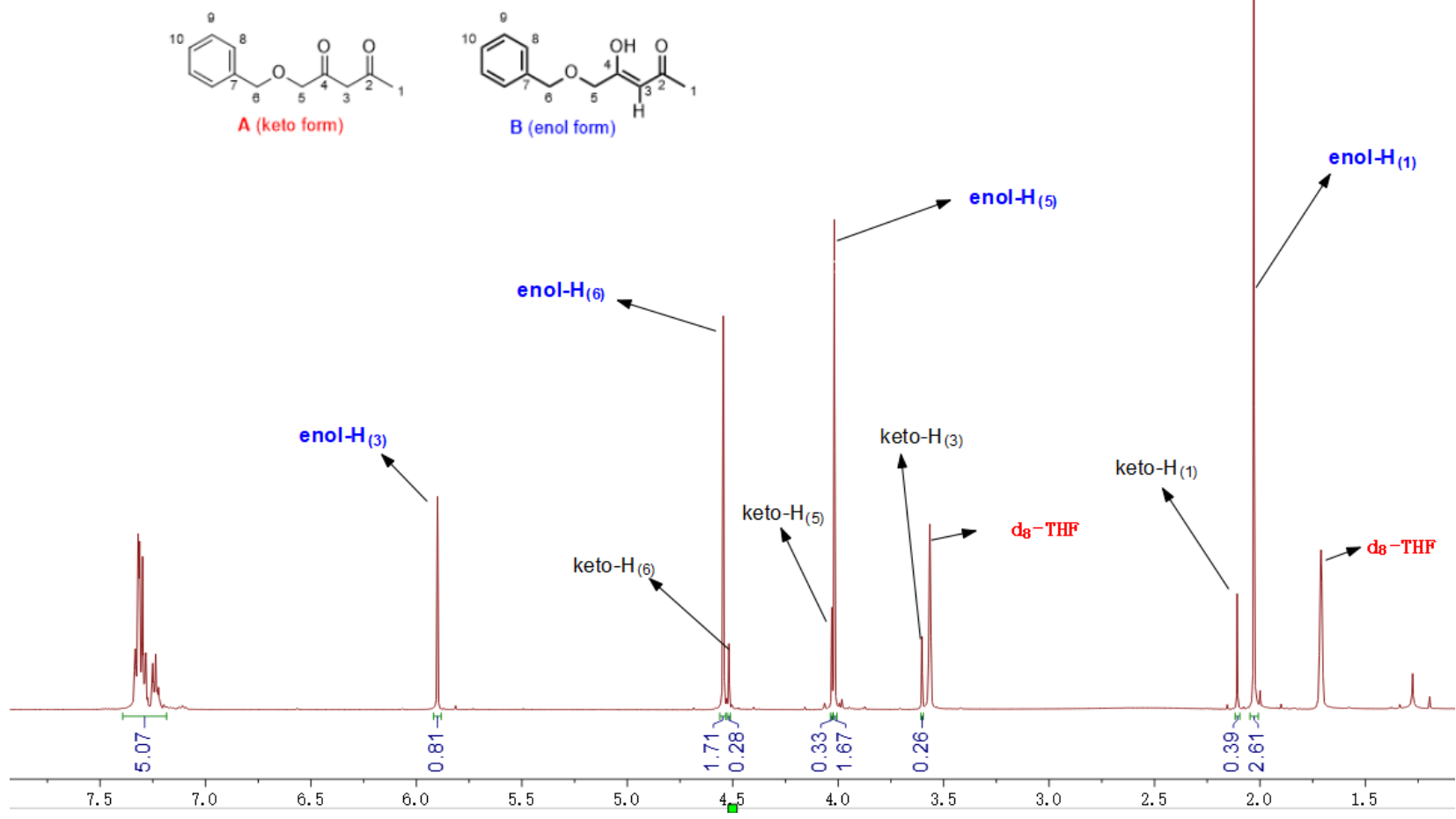

Figure S1. The Assignments of the ${ }^{1} \mathrm{H}$ NMR $\left(d_{8}\right.$-THF, $\left.500 \mathrm{MHz}\right)$ Spectrum of 1 a.

From the ${ }^{1} \mathrm{H}_{-}{ }^{13} \mathrm{C}-\mathrm{HMBC}$ spectrum, the enol form $\mathbf{B}$ is the predominant in THF. 


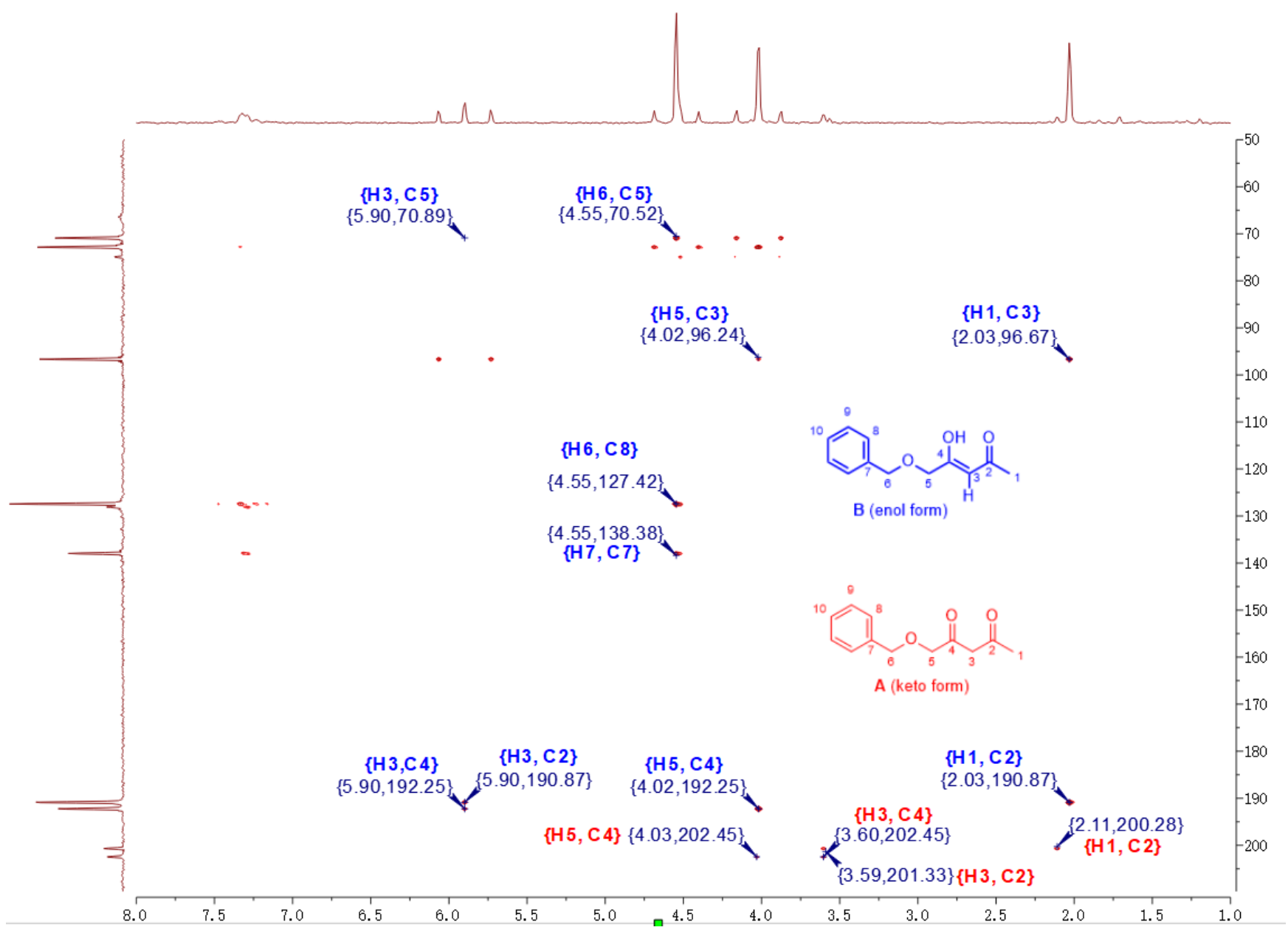

Figure S2. ${ }^{1} \mathrm{H}_{-}{ }^{13} \mathrm{C}$ HMBC $\left(d_{8}-\mathrm{THF}, 500 \mathrm{MHz}\right)$ Correlations for $\mathbf{1 a}$.

\section{References}

[1] W. Wenner, J. T. Plati, J. Org. Chem. 1946, 11, 751-759.

[2] B. C. Ranu, S. Bhar, R. Chakraborti, J. Org. Chem. 1992, 57, 7349-7352.

[3] D. Globisch, C. A. Lowery, K. C. McCague, K. D. Janda, Angew. Chem., Int. Ed. 2012, 51, 4204-4208, Supporting Information.

[4] V. I. Dulenko, S. V. Tolkunov (Inst. Fiz.-Org. Khim. Uglekhim.), WO 2009015369, 1987; [Chem. Abstr. 1987, 108, 167343].

[5] A. K. Chakraborti, Basak, V. Grover, J. Org. Chem. 1999, 64, 8014-8017.

[6] G. Solladié, F. Colobert, D. Denni, Tetrahedron: Asymmetry 1998, 9, 3081-3094.

[7] D. A. Evans, M. C. Kozlowski, J. A. Murry, C. S. Burgey, K. R. Campos, B. T. Connell, R. J. Staples, J. Am. Chem. Soc. 1999, 121, 669-685, in Supporting Information.

[8] O. Labeeuw, J. B. Bourg, P. Phansavath, J. P. Genêt, Arkivoc 2007, 2007, 94-106.

[9] a) S. Kwiatkowski, J. Chem. Soc., Chem. Commun. 1987, 1496-1498; b) S. Kwiatkowski, S. Ostrowski, Bull. Soc. Chim. Belg. 1993, 102, 259-269. 
[10] K.-H. Park, W. J. Marshall, Tetrahedron Lett. 2004, 45, 4931-4934.

[11] R. E. Bowman, D. J. Tivey, J. Chem. Soc. (Resumed) 1954, 4548-4550.

[12] S. Wang, J. Golec, W. Miller, S. Milutinovic, A. Folkes, S. Williams, T. Brooks, K. Hardman, P. Charlton, S. Wren, J. Spencer, Bioorg. Med. Chem. Lett. 2002, 12, 2367-2370.

[13] a) J. J. Eisch, S. K. Dua, M. Behrooz, J. Org. Chem. 1985, 50, 3674-3676; b) G. Solladie, N. Ghiatou, Tetrahedron: Asymmetry 1992, 3, 33-38.

[14] I. Katsuyama, S. Ogawa, Y. Yamaguchi, K. Funabiki, M. Matsui, H. Muramatsu, K. Shibata, Synthesis 1997, 13211324 .

[15] J. Zhao, Y. Zhao, H. Fu, Angew. Chem. Int. Ed. 2011, 50, 3769-3773.

[16] A. K. Roy, S. Batra, Synthesis 2003, 2325-2330.

[17] J. B. Kraïem, H. Amri, Synth. Commun. 2012, 43, 110-117.

[18] J. Wang, N. A. Morra, H. Zhao, J. S. T. Gorman, V. Lynch, R. McDonald, J. F. Reichwein, B. L. Pagenkopf, Can. J. Chem. 2009, 87, 328-334.

[19] K. Funabiki, Y. Itoh, Y. Kubota, M. Matsui, J. Org. Chem. 2011, 76, 3545-3550.

[20] H. Li, C.-S. Da, Y.-H. Xiao, X. Li, Y.-N. Su, J. Org. Chem. 2008, 73, 7398-7401.

[21] J.-F. Zhao, B.-H. Tan, T.-P. Loh, Chem. Sci. 2011, 2, 349-352.

[22] J. Kang, Y. H. Kim, M. Park, C. H. Lee, W.-J. Kim, Synth. Commun. 1984, 14, 265-269.

[23] Drexler, E. J.; Field, K. W. J. Chem. Educ. 1976, 53, 392-393.

[24] Tan, M.; Bİldİri̇ci̇, İ.; Mengeş, N. J. Serb. Chem. Soc. 2018, 83, 953-968.

\section{NMR spectra of key substrates and all hydrogenation products}


1a

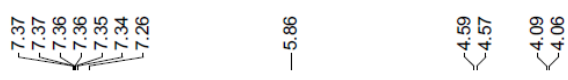

กูก

$\stackrel{8}{i}$

BnO

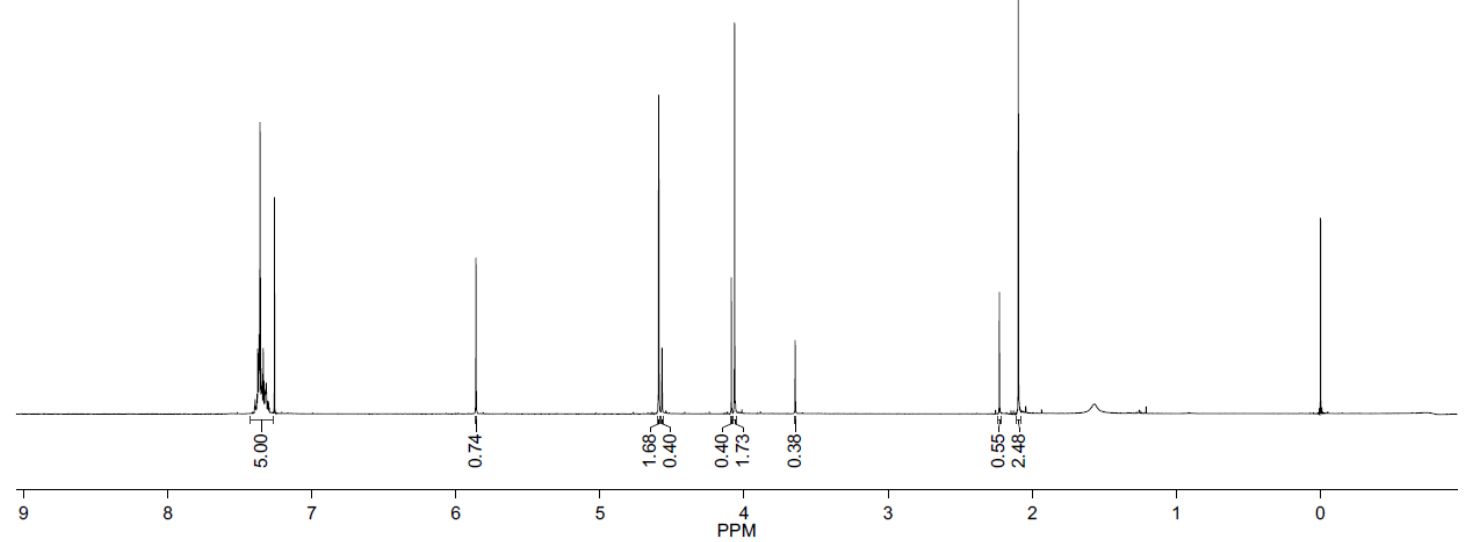

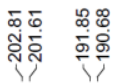

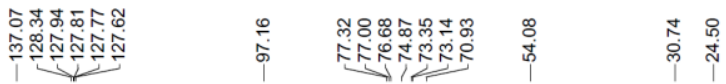

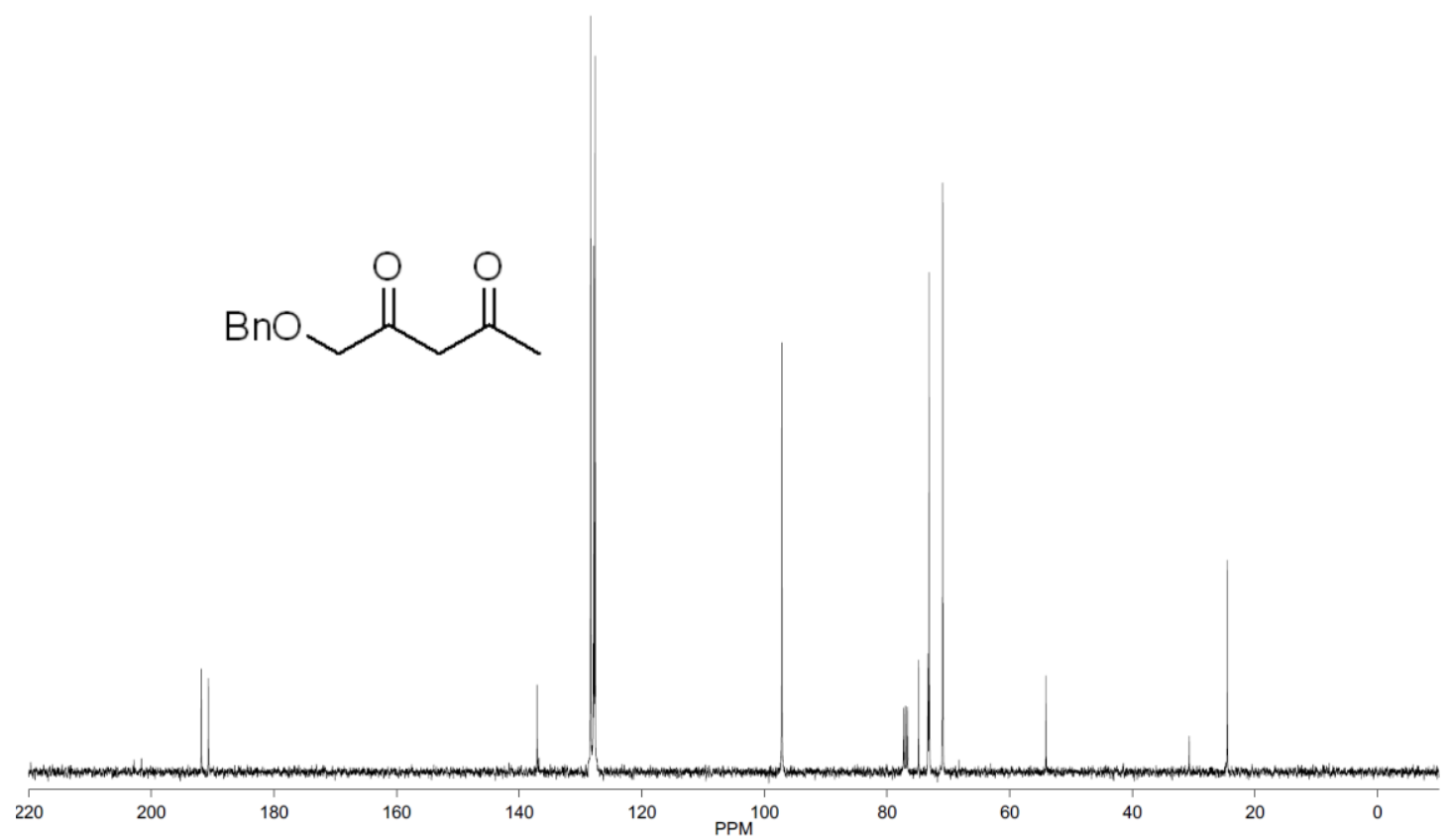


$1 b$

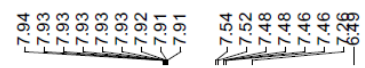

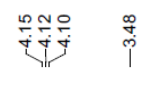

$\stackrel{8}{i}$
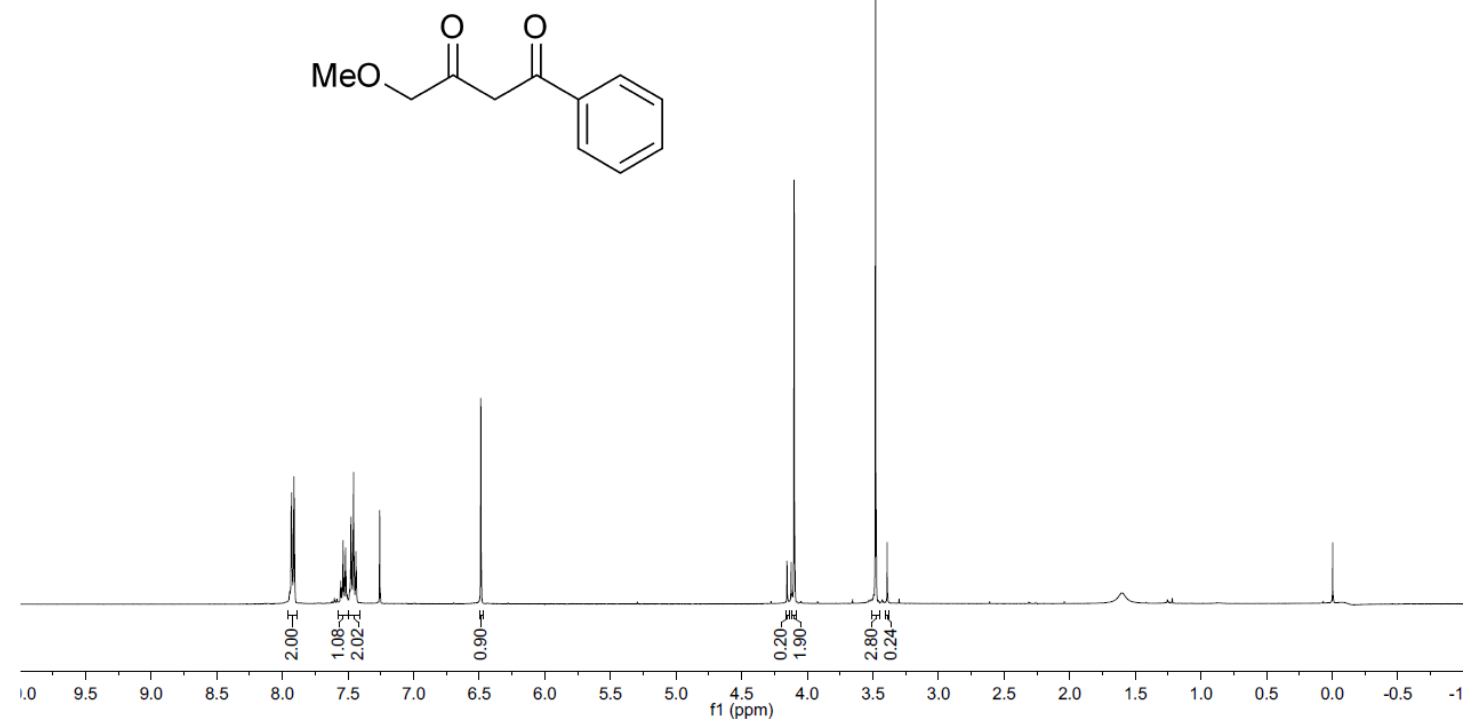

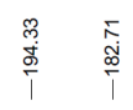

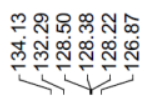

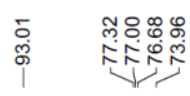

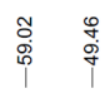

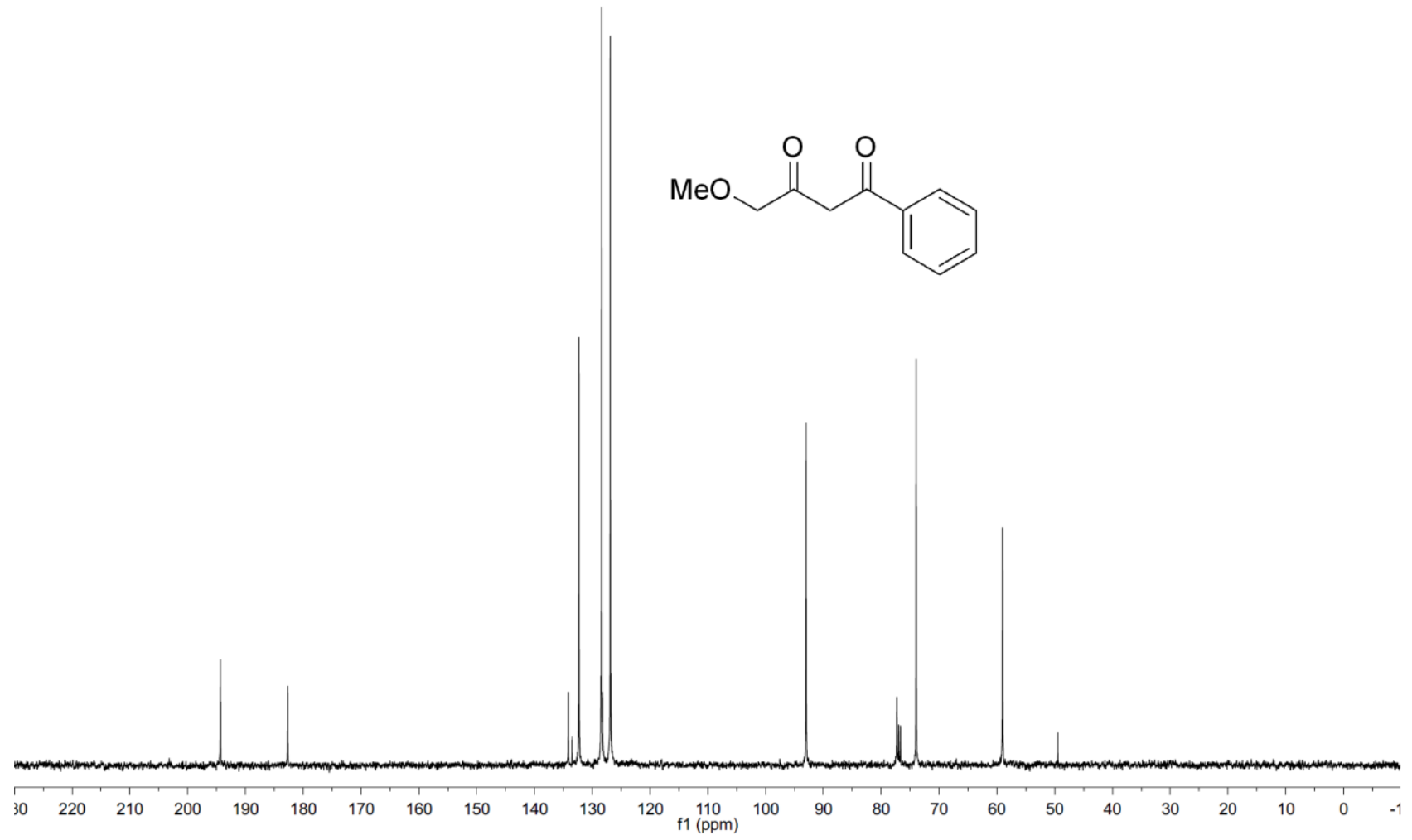

21 


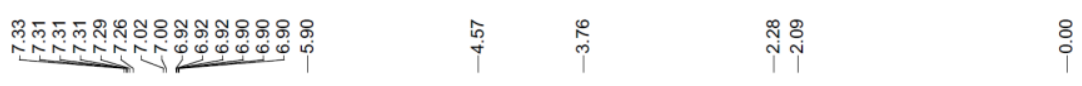

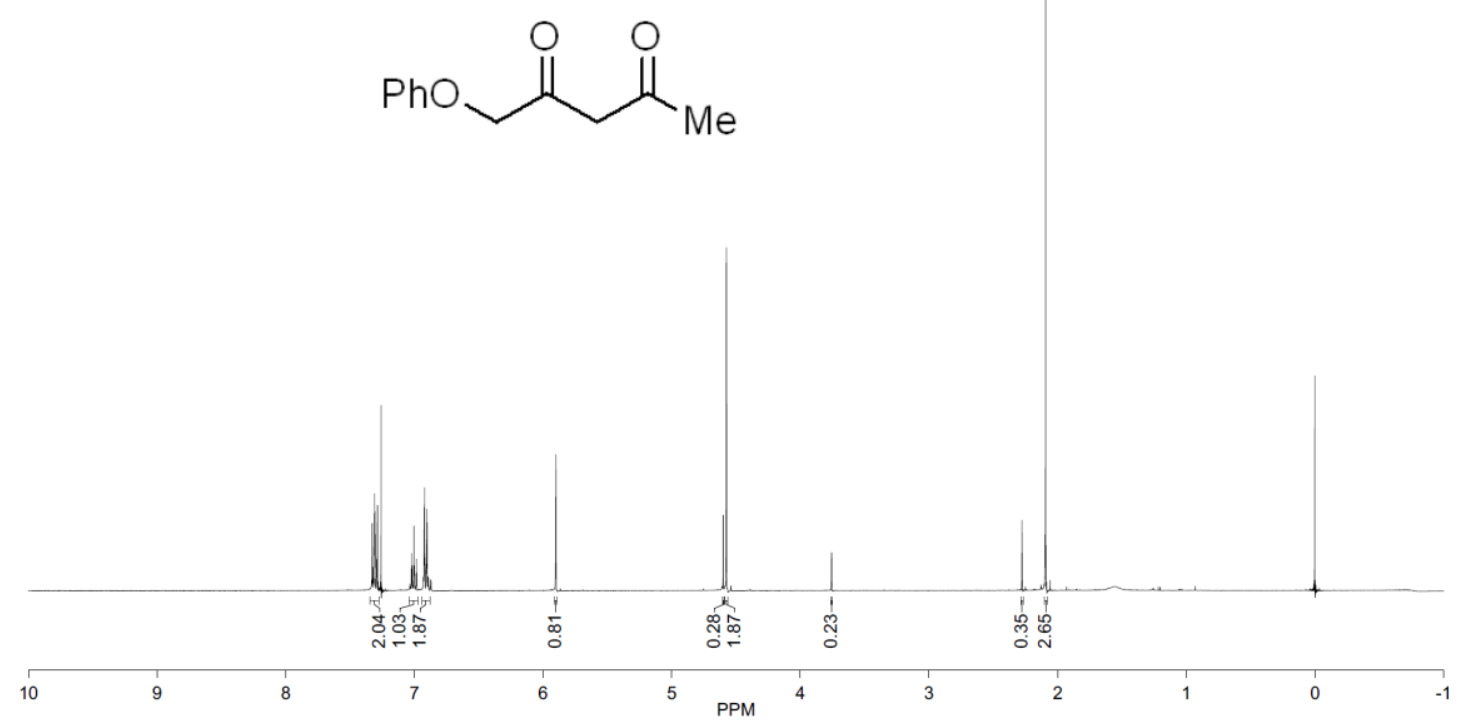

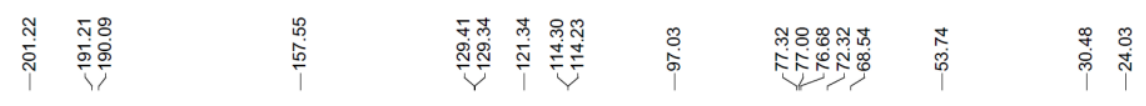

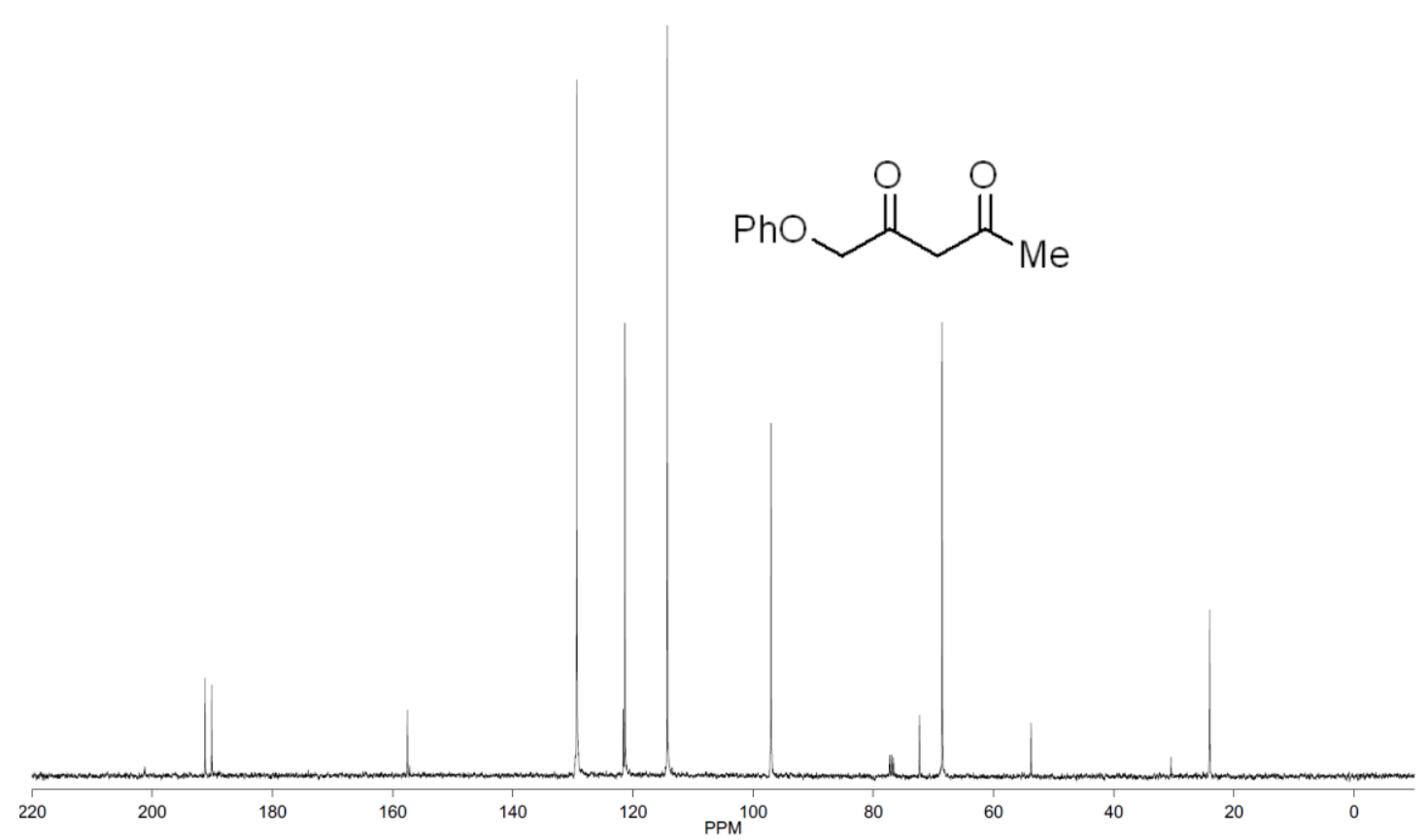



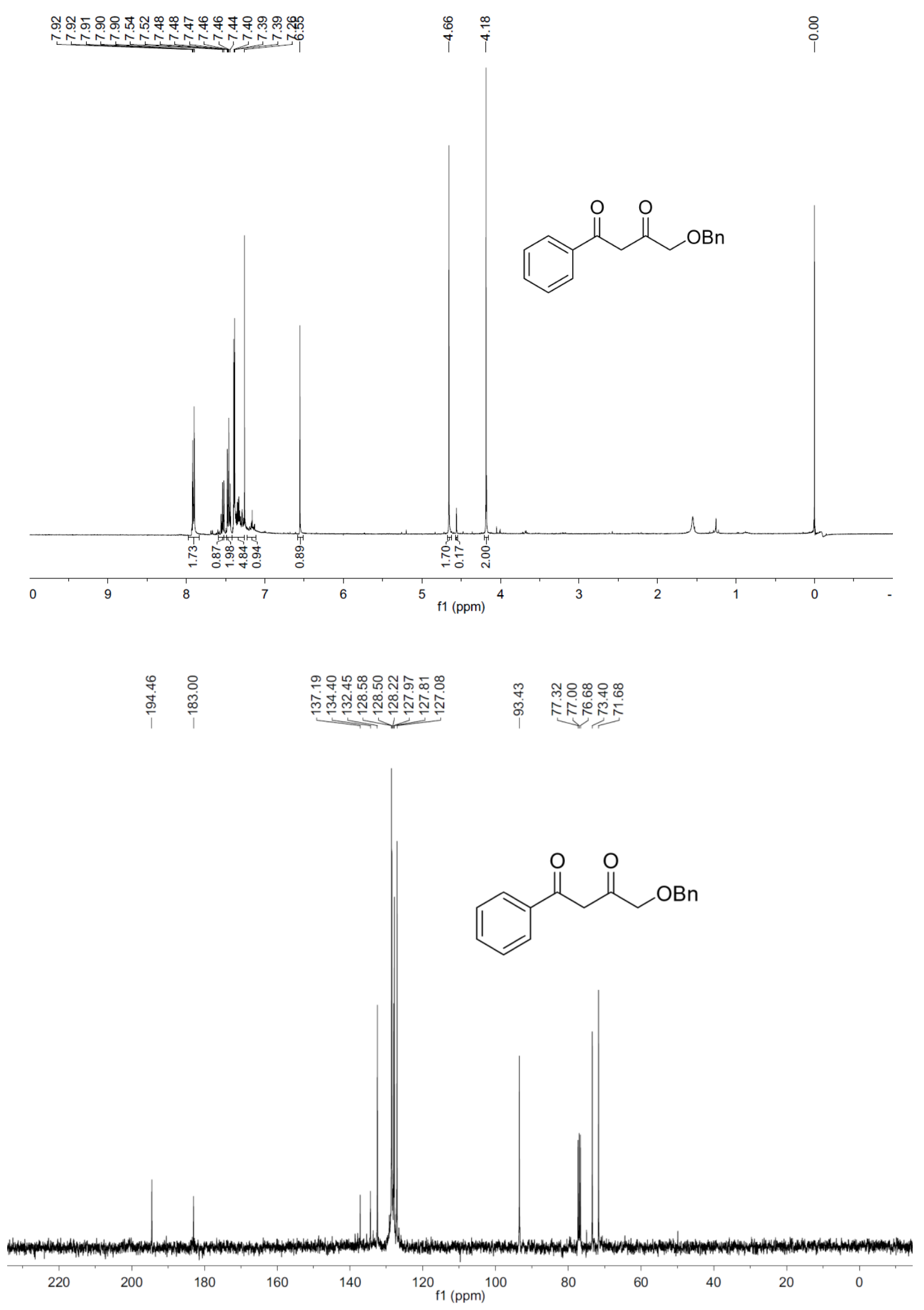
1e

量

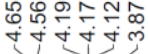

$\stackrel{\circ}{\circ}$

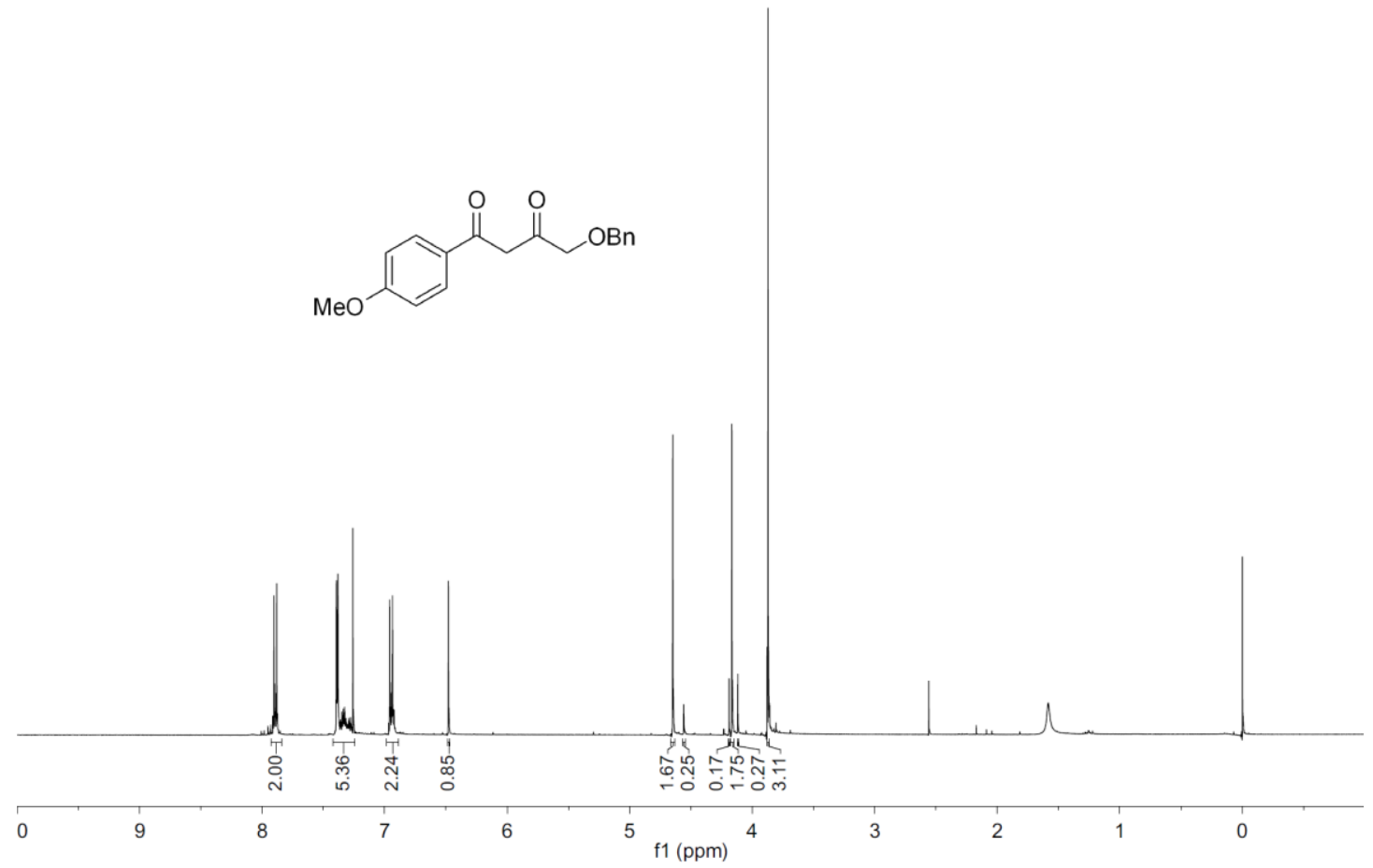

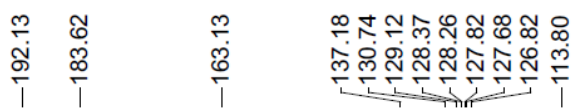

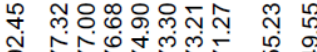

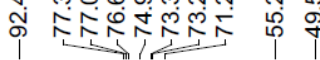

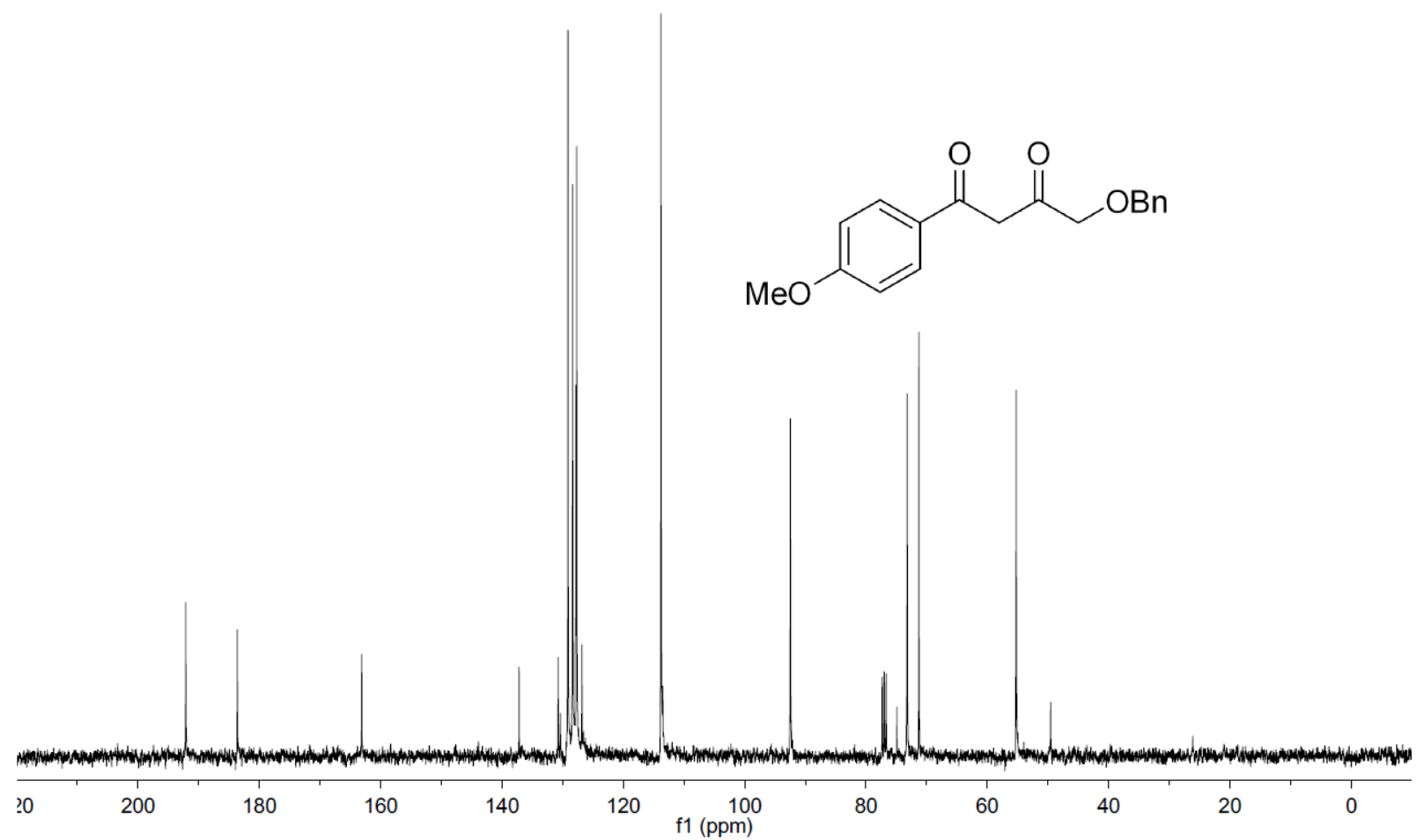




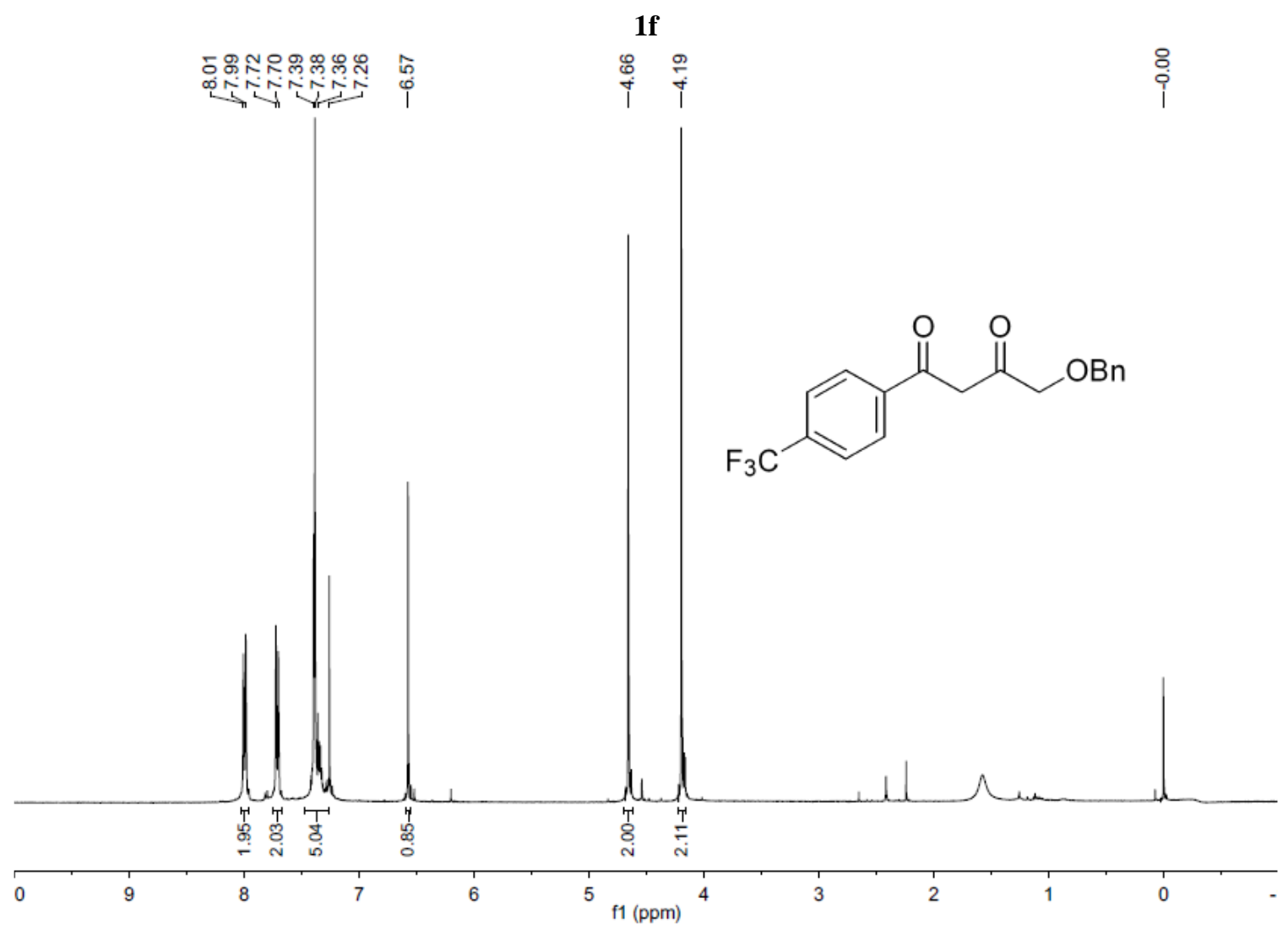

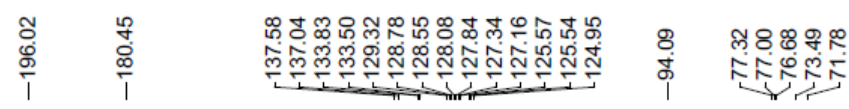

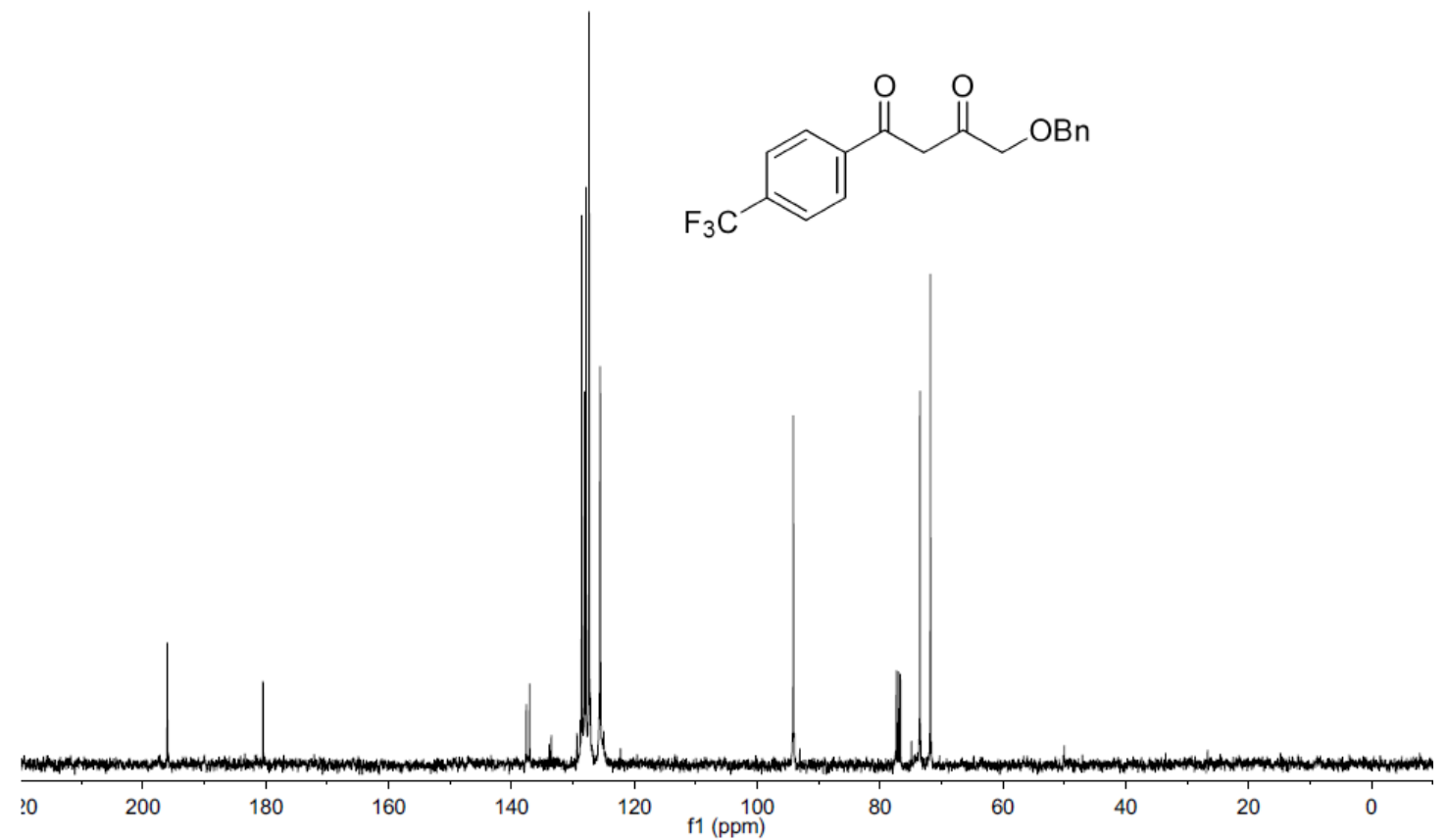




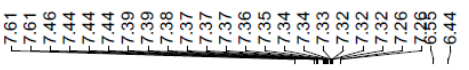

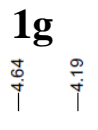

$\stackrel{8}{1}$

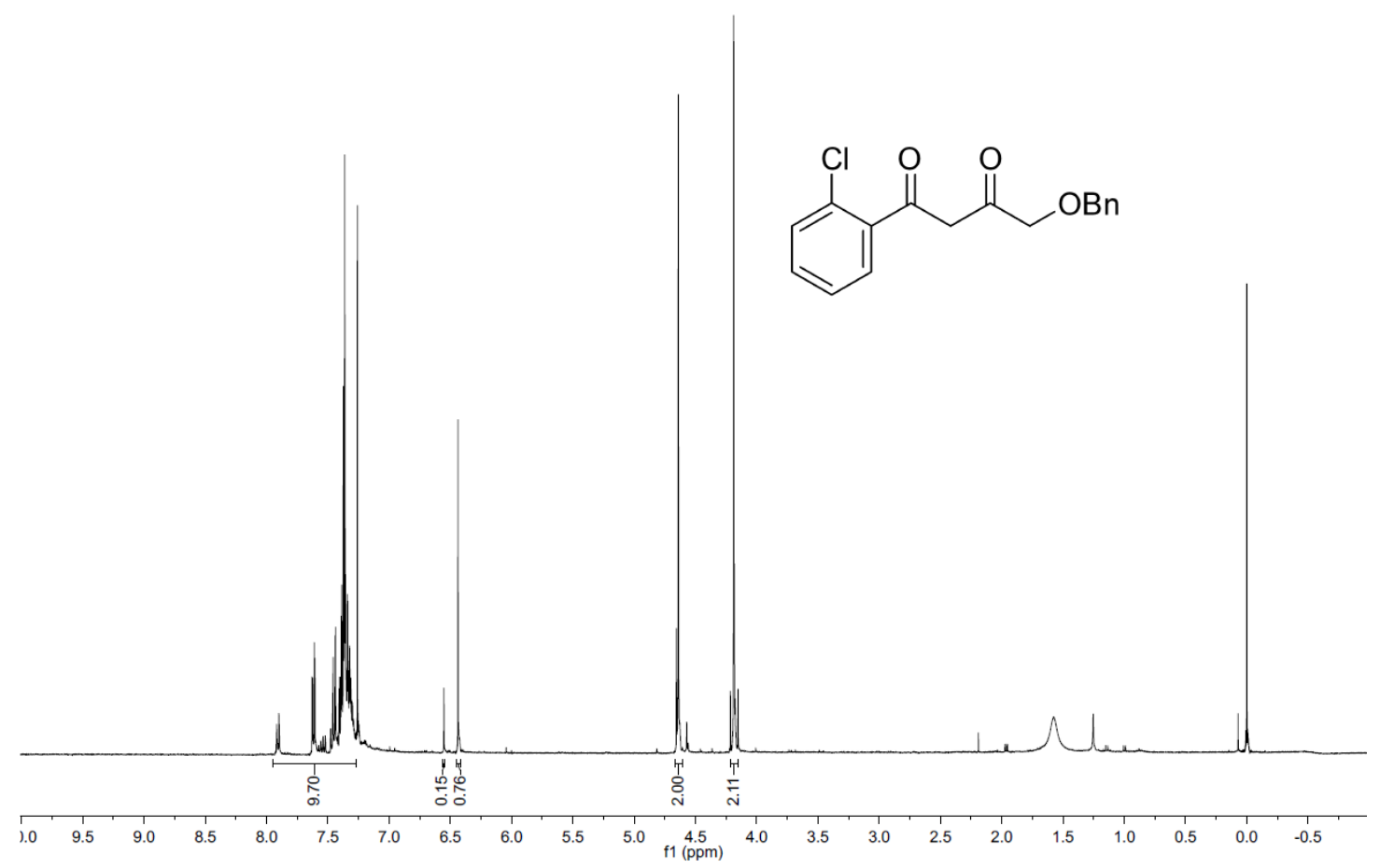

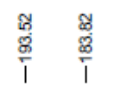

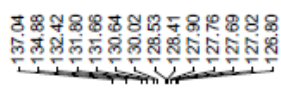

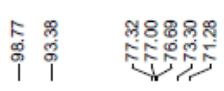

虽

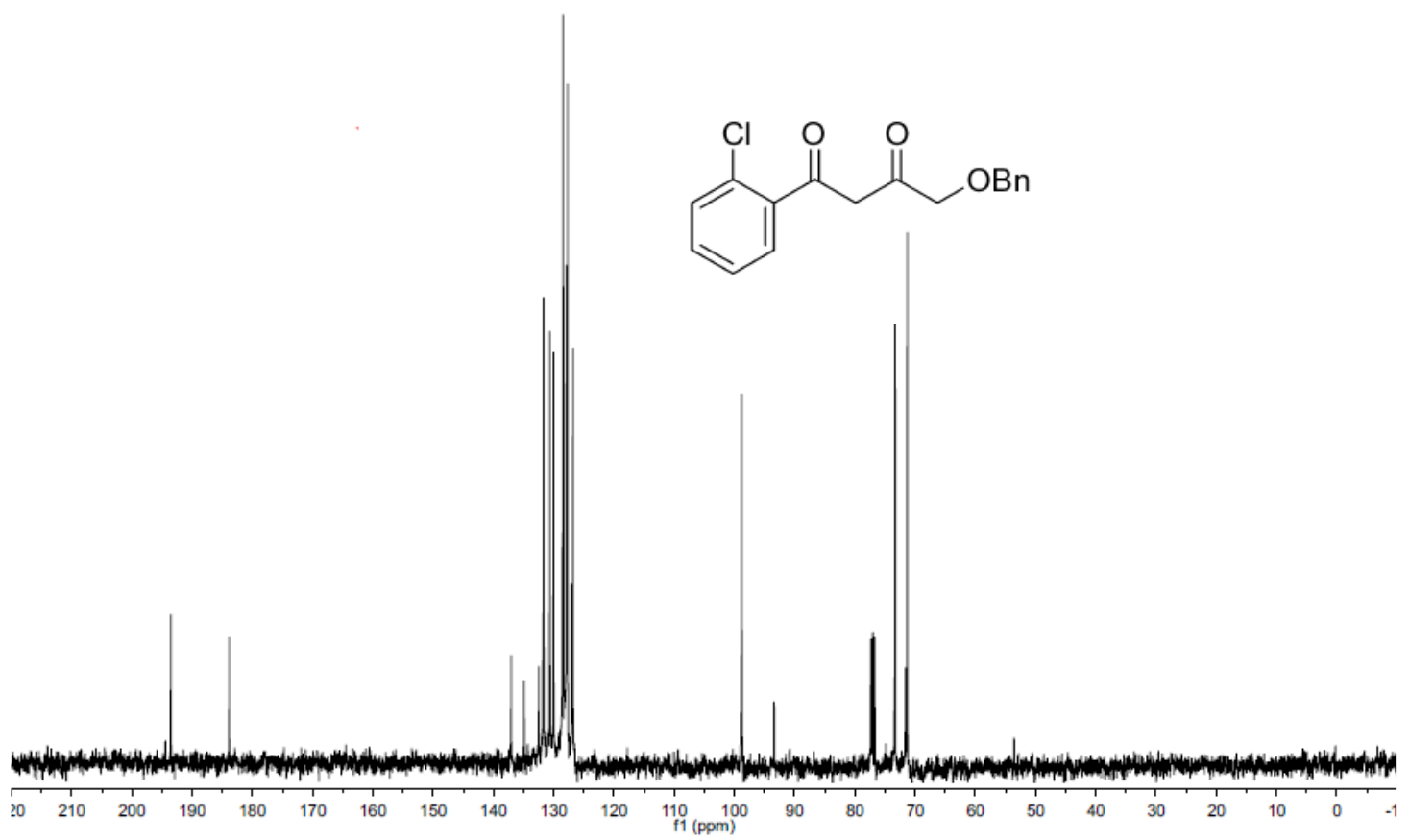

26 


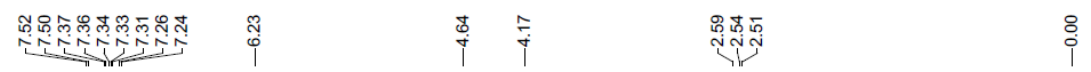

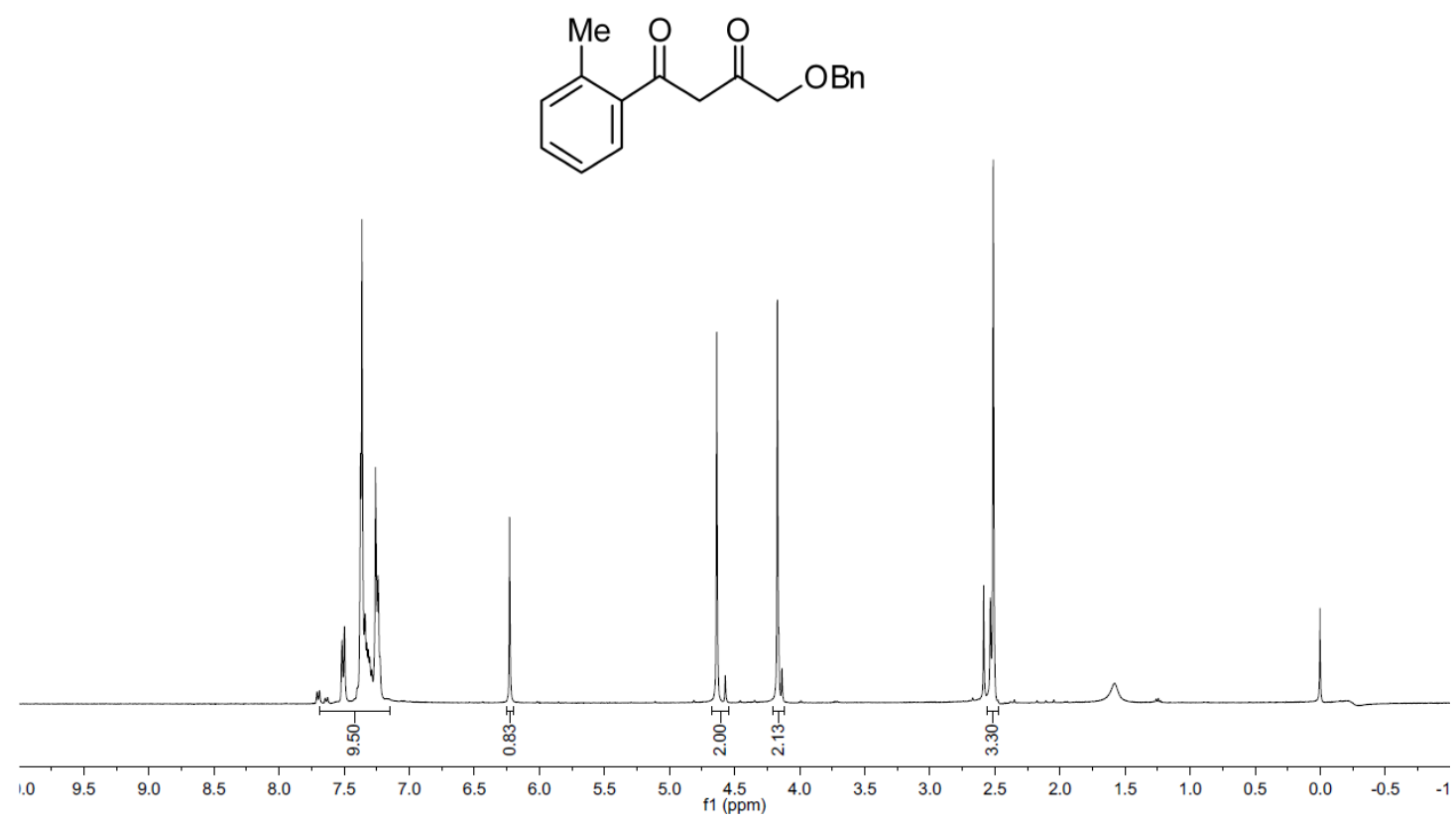

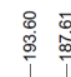

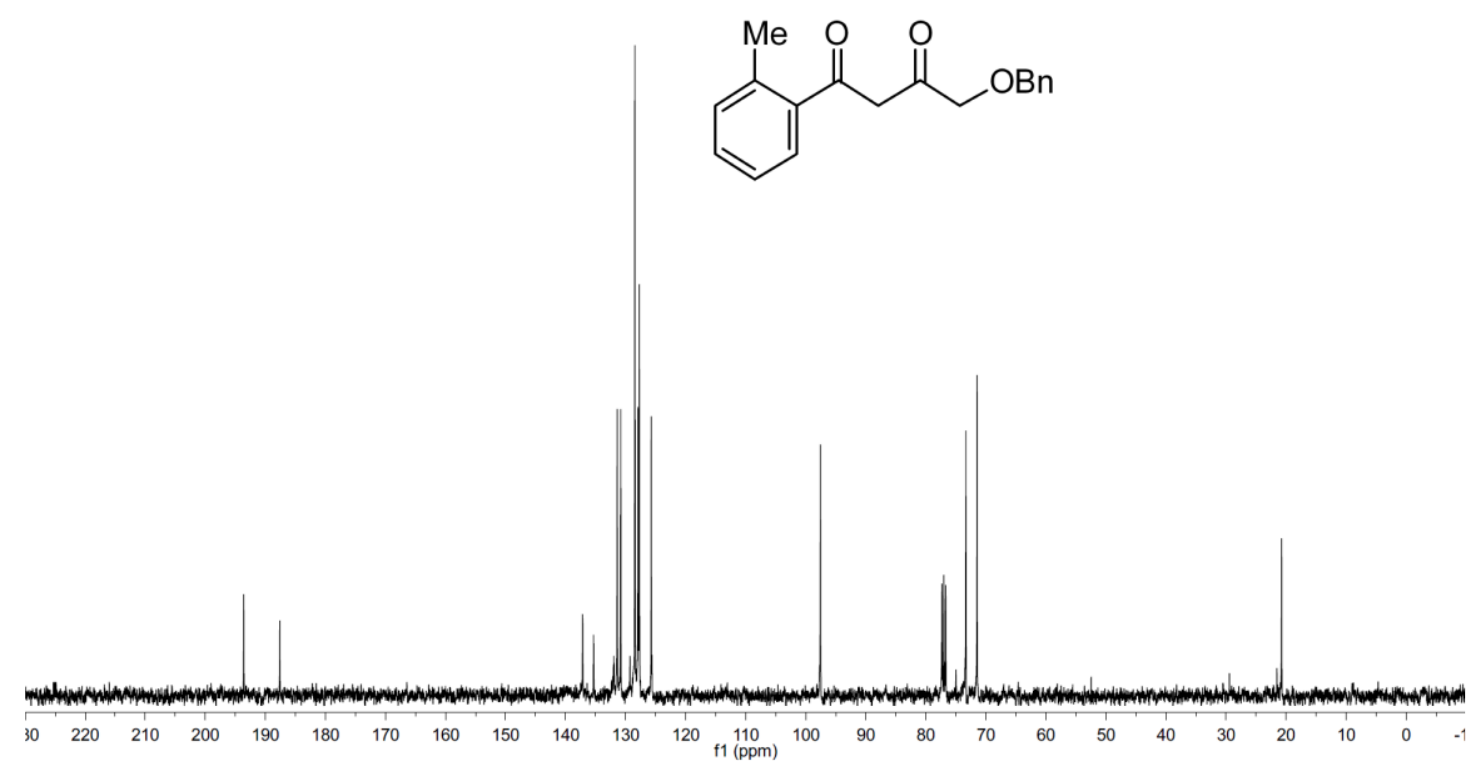



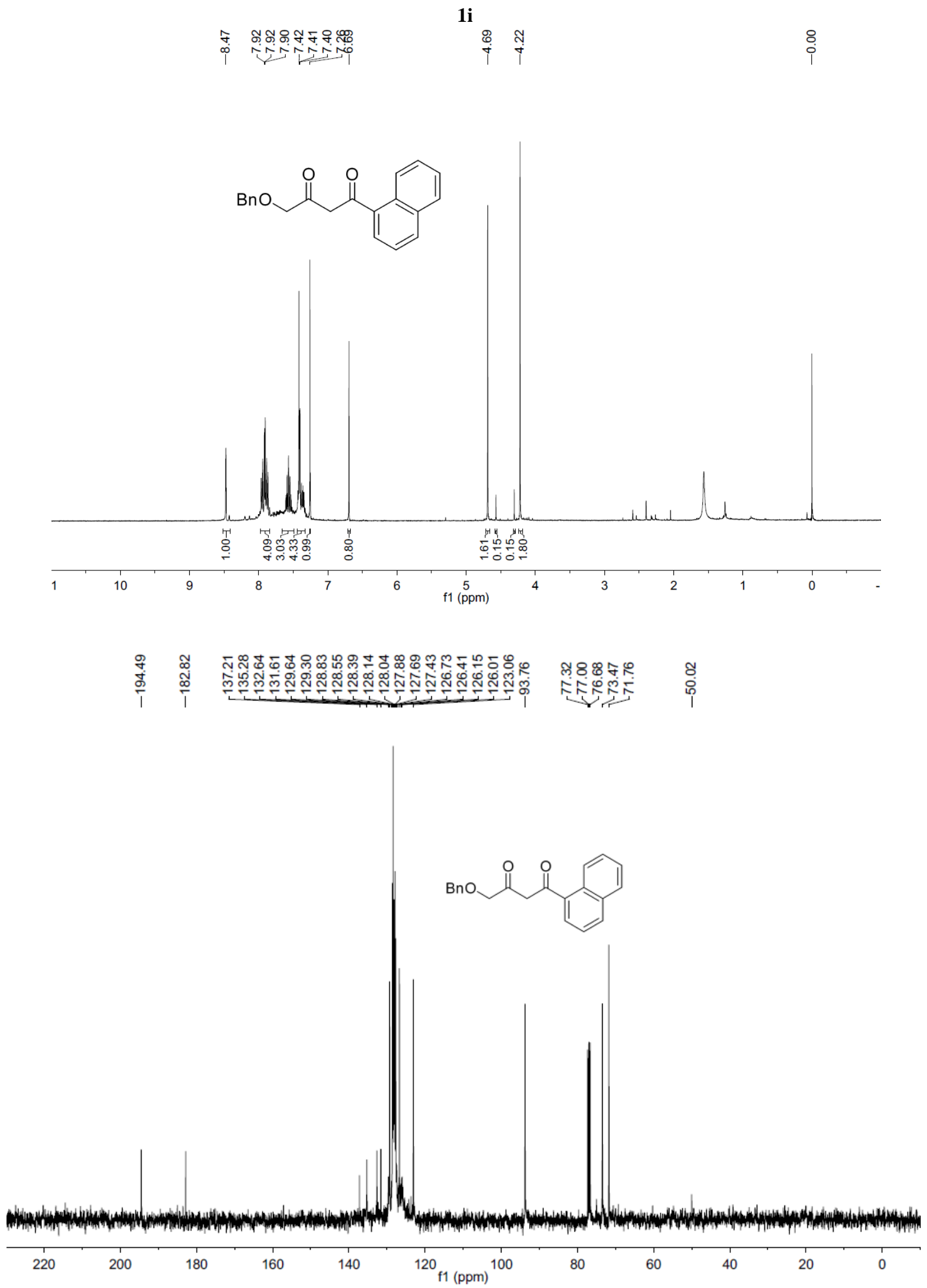


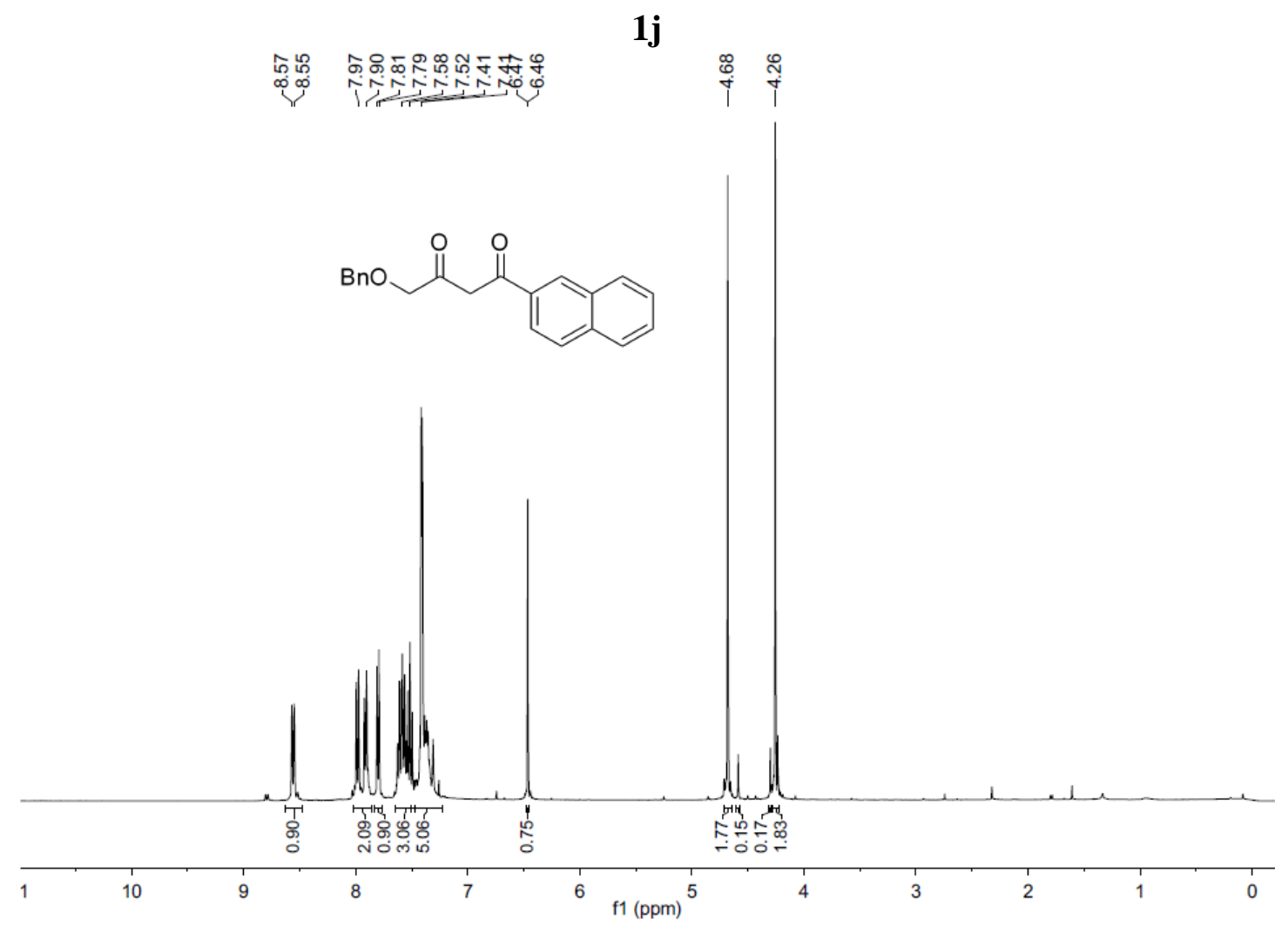

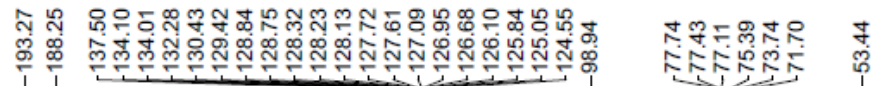

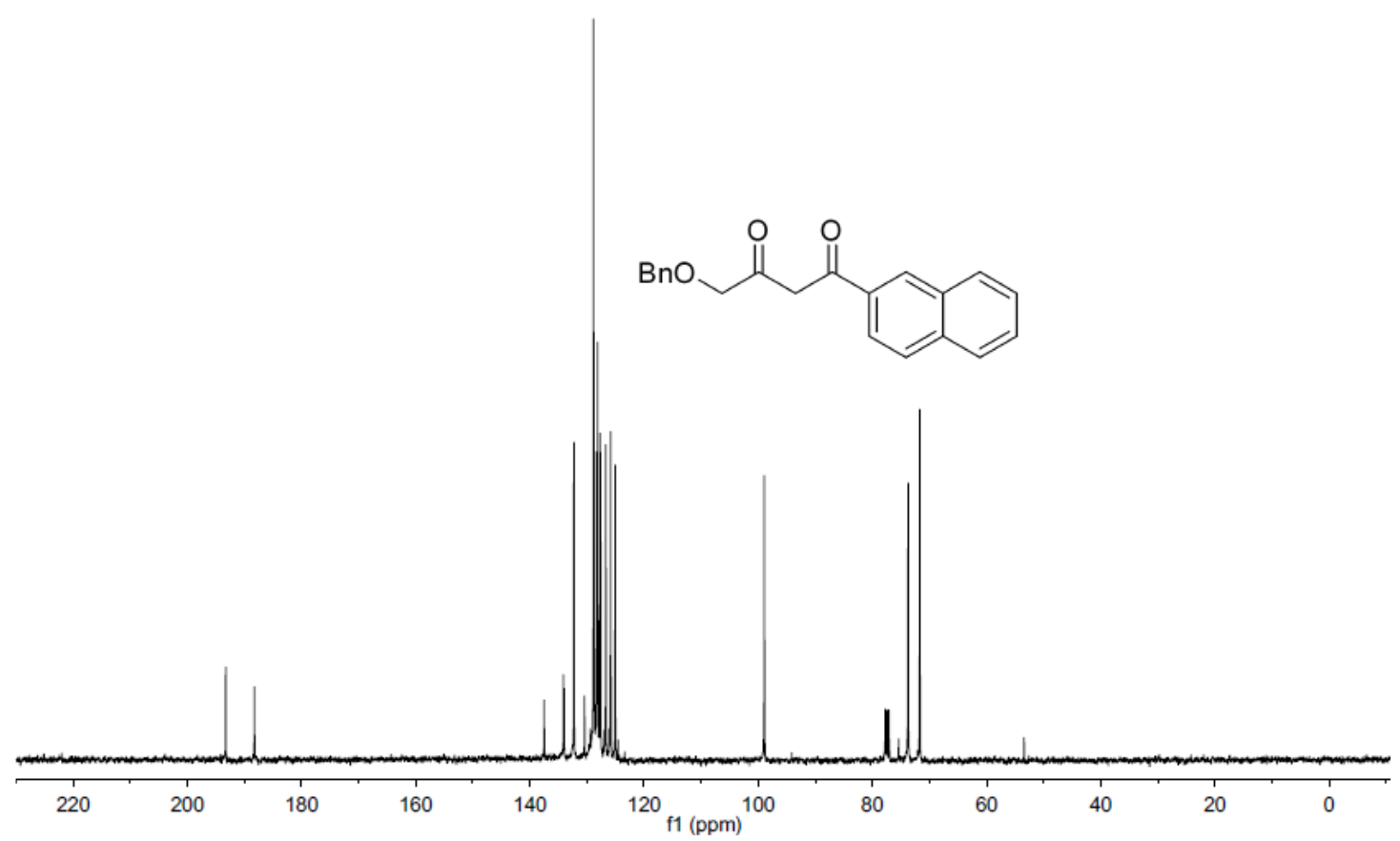


$1 \mathrm{k}$

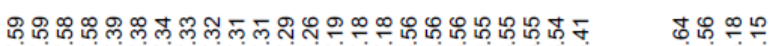

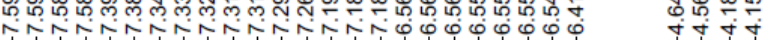

$\stackrel{\infty}{\sim} \stackrel{\infty}{\sim} \stackrel{\infty}{\sim}$

$\stackrel{\circ}{i}$

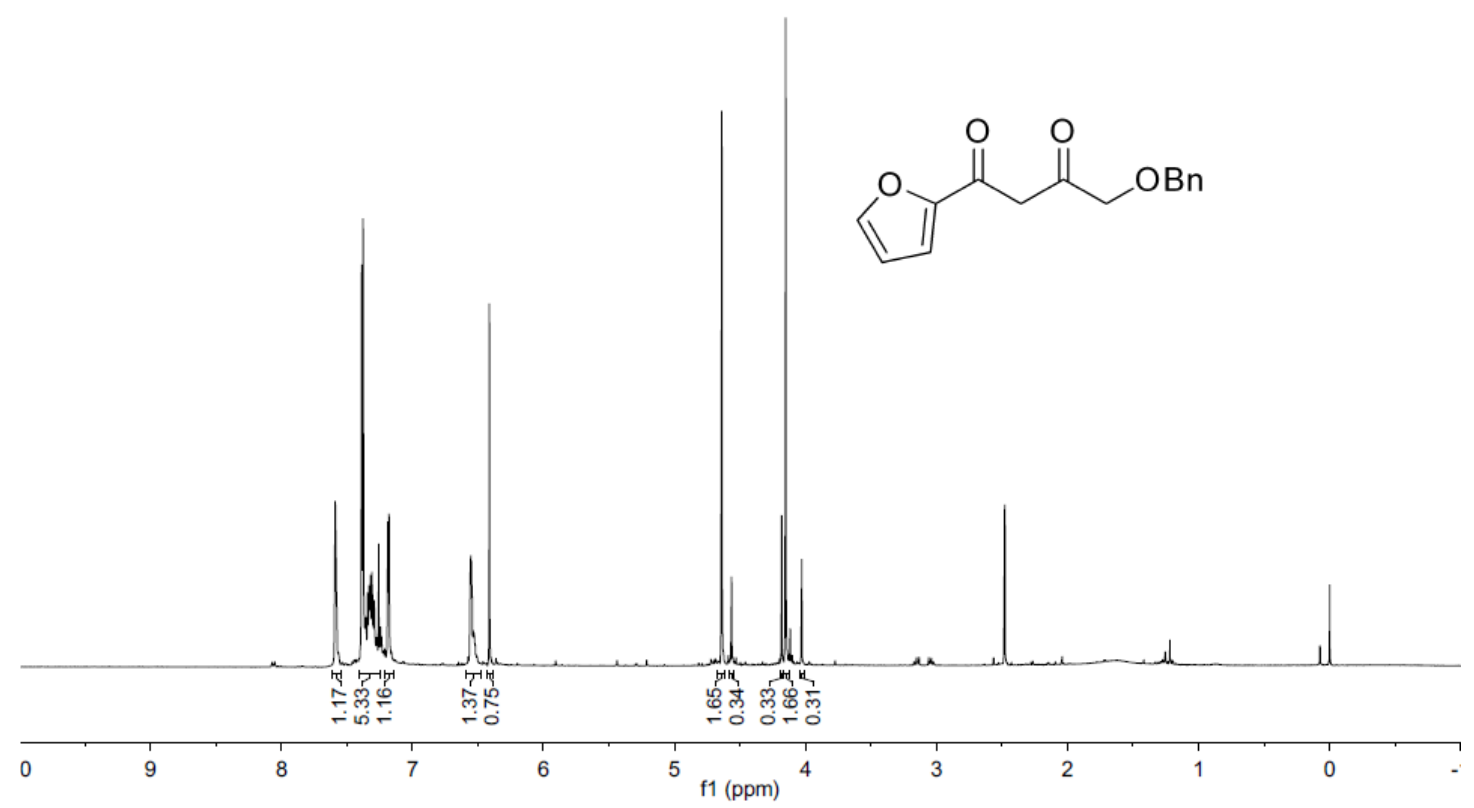

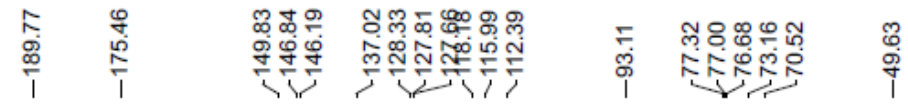

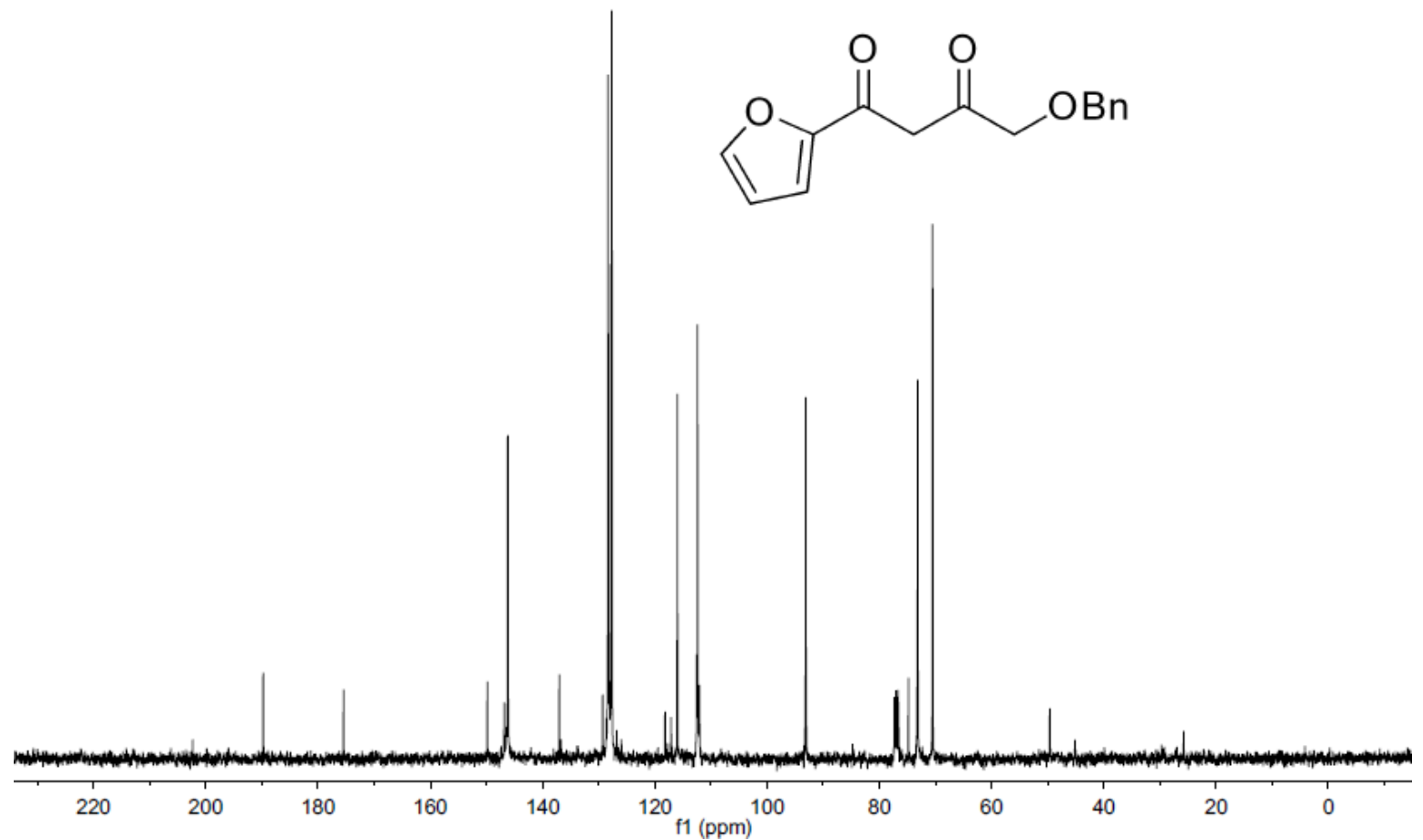


$2 a$

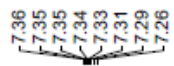

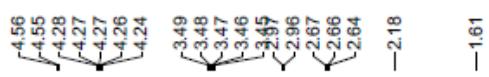

$\stackrel{\circ}{i}$

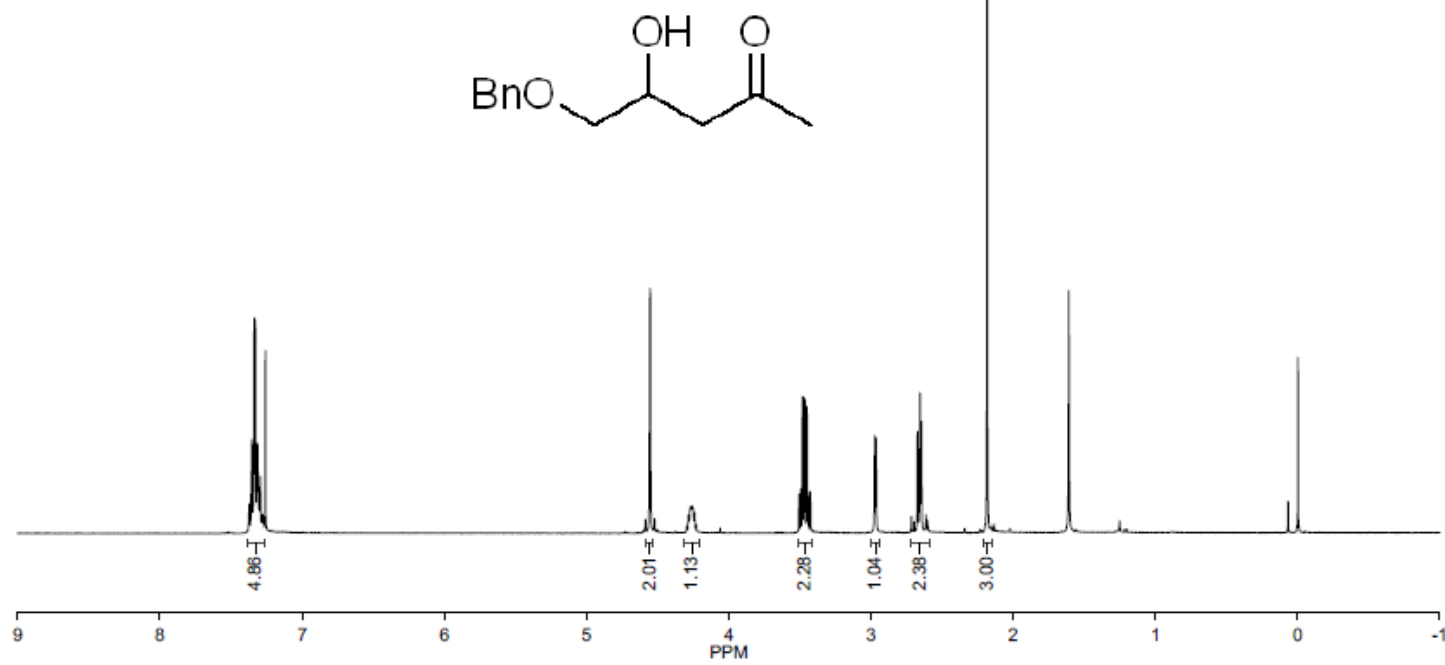

ֻั

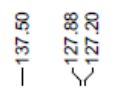

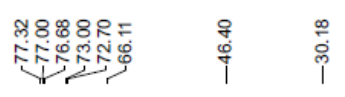

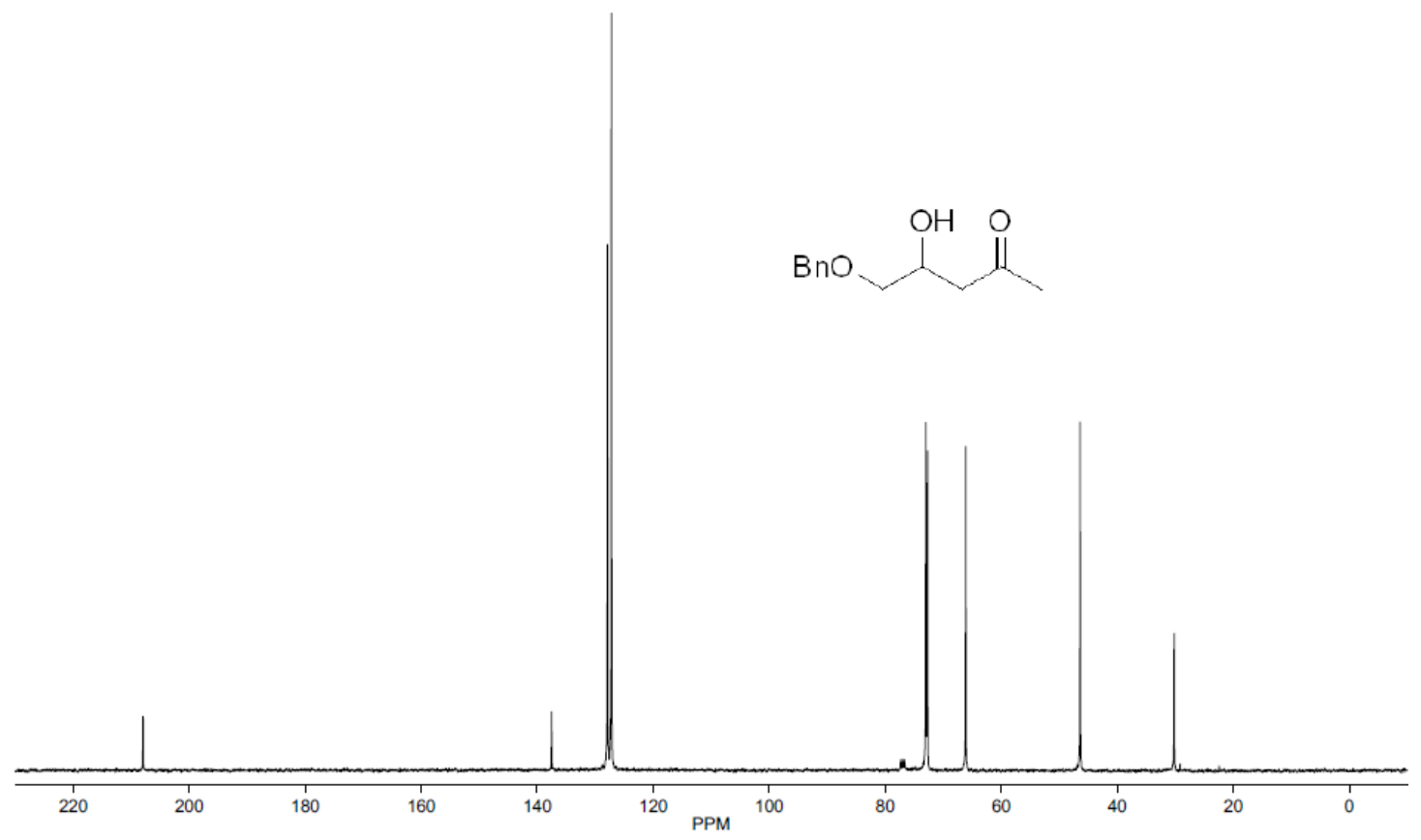



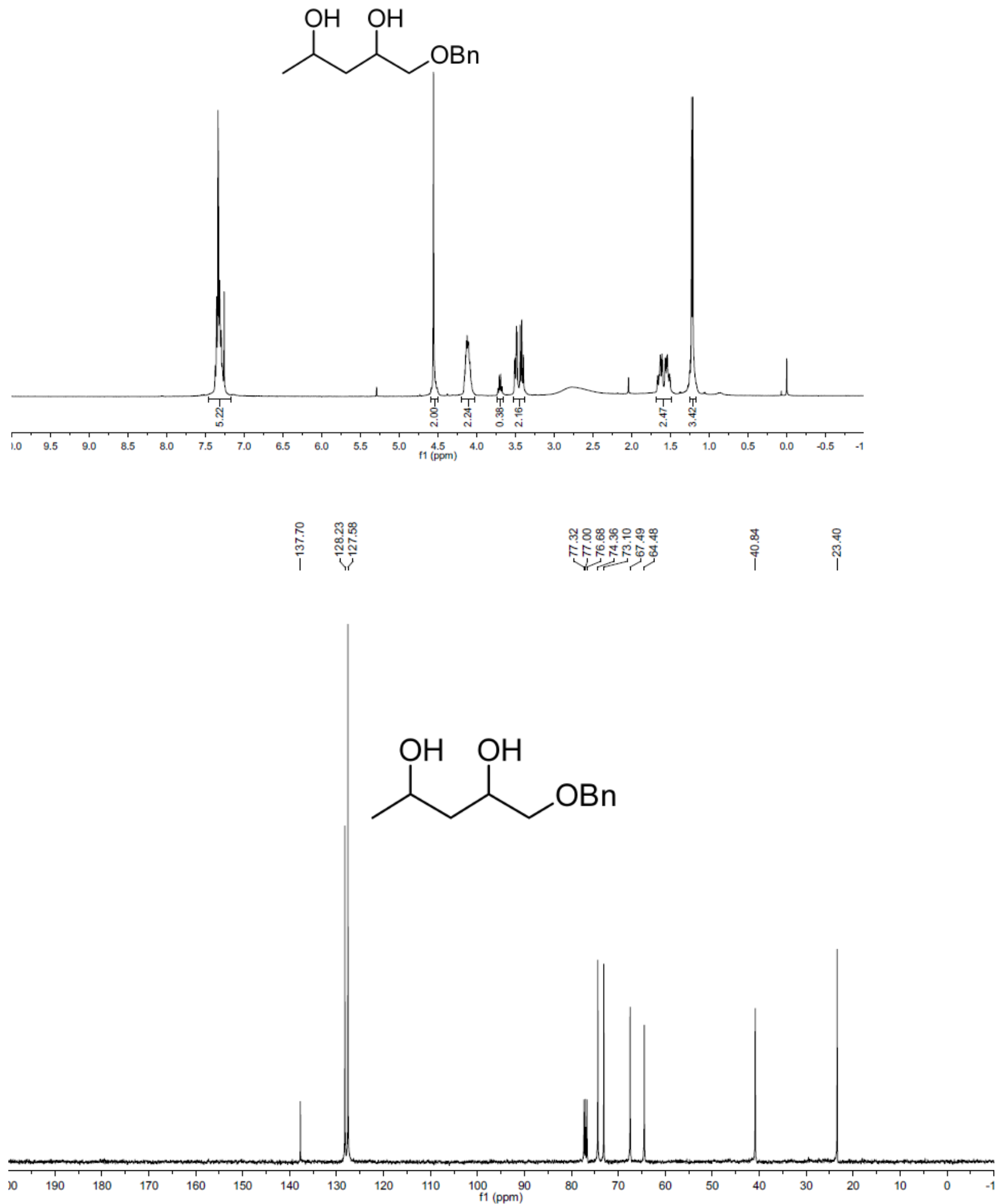
$2 \mathrm{~b}$

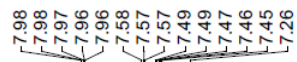

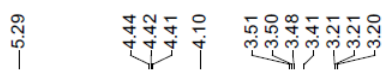

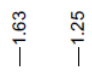

¿̀
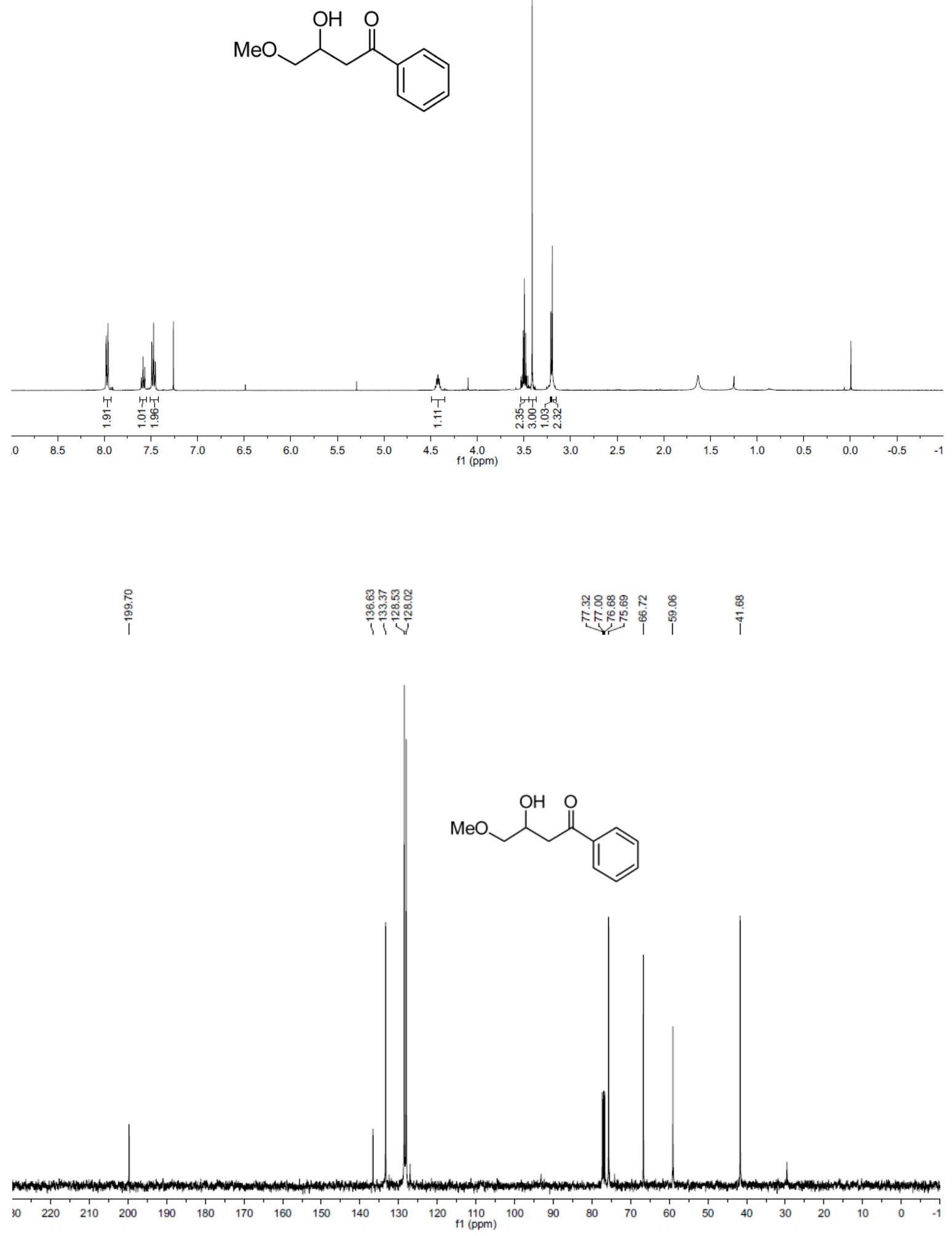

33 
2c

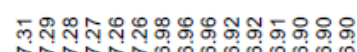

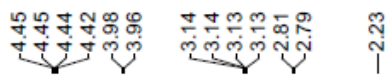

$\stackrel{8}{i}$<smiles>CC(=O)CC(O)COc1ccccc1</smiles>

ले

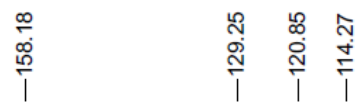

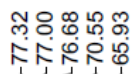

$\begin{array}{ll}9 & 9 \\ 0 & 0 \\ 0 & 0 \\ 0 & 0\end{array}$

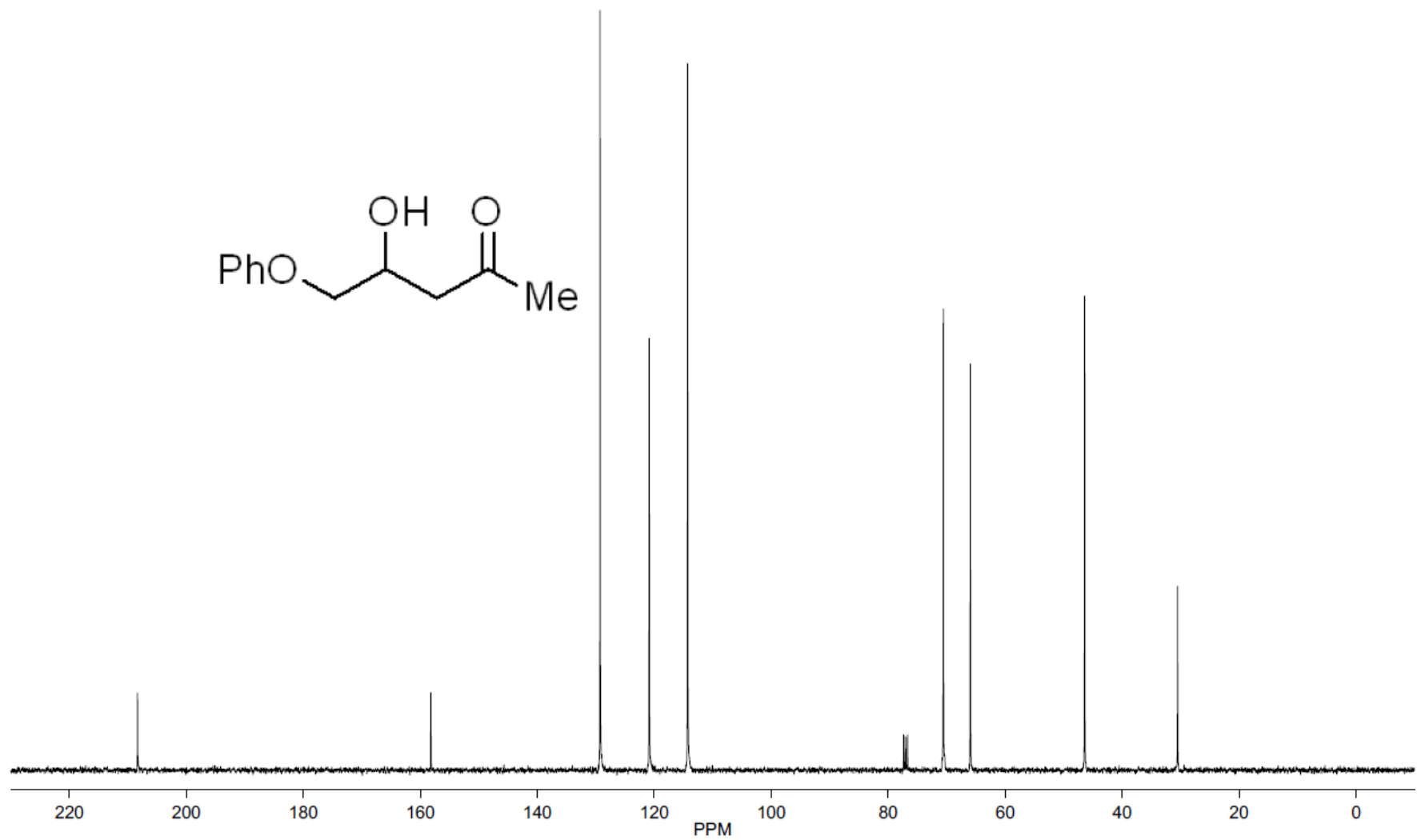


2d
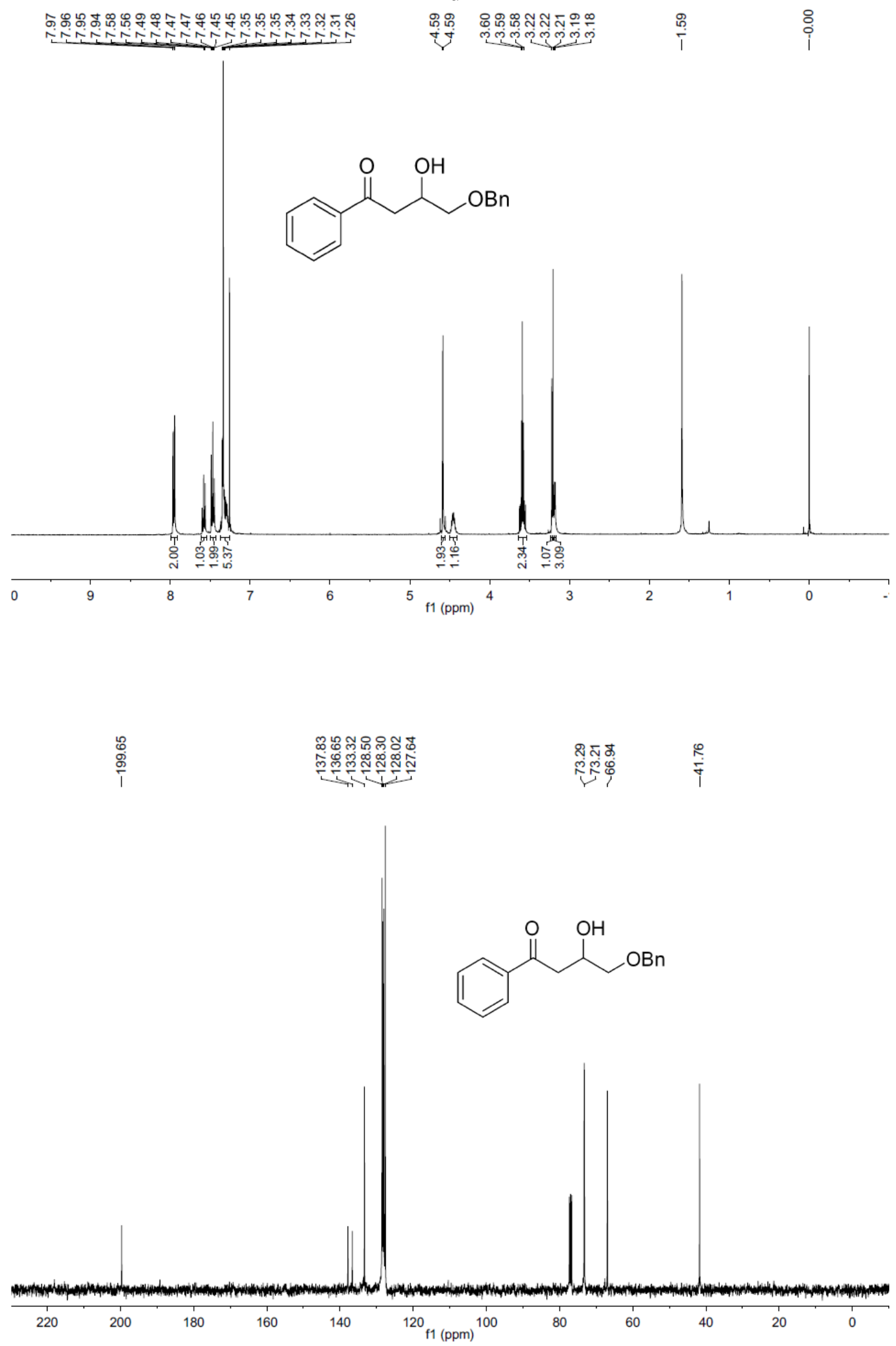
$2 \mathrm{e}$

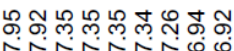

我舟

* लंखुलुल

$\stackrel{8}{i}$

(N)

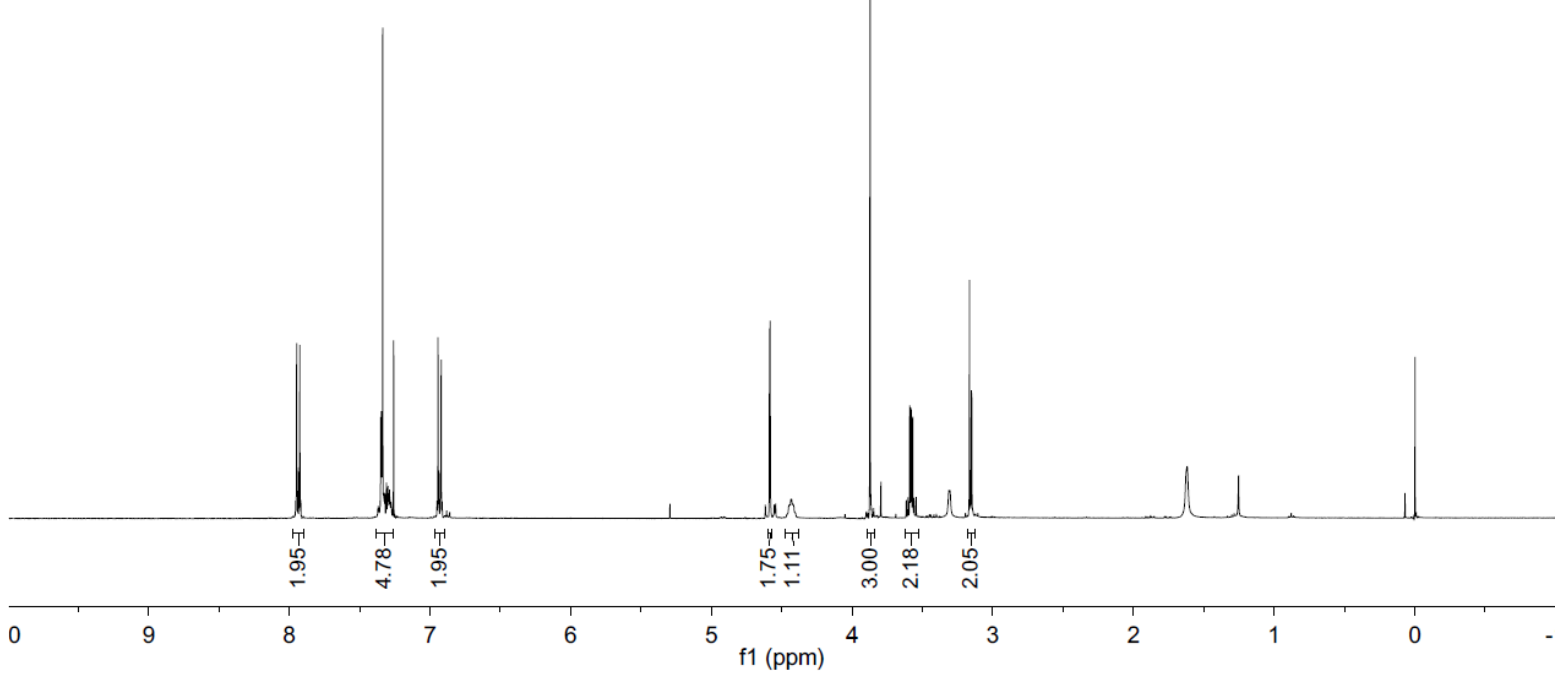

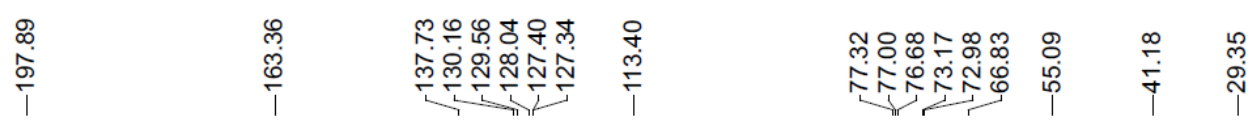

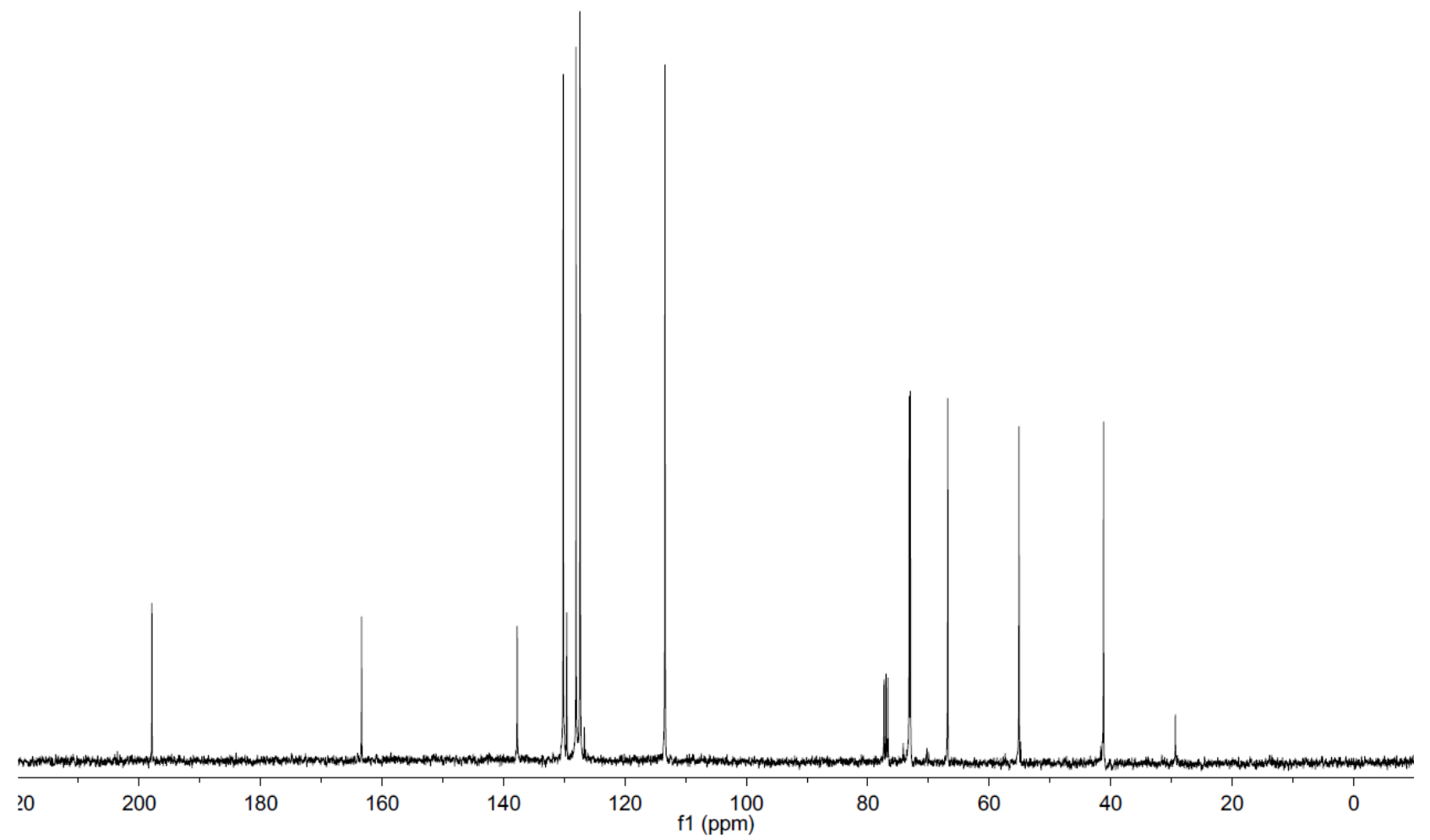




$$
\text { 2f }
$$

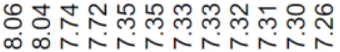

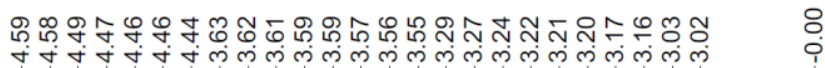
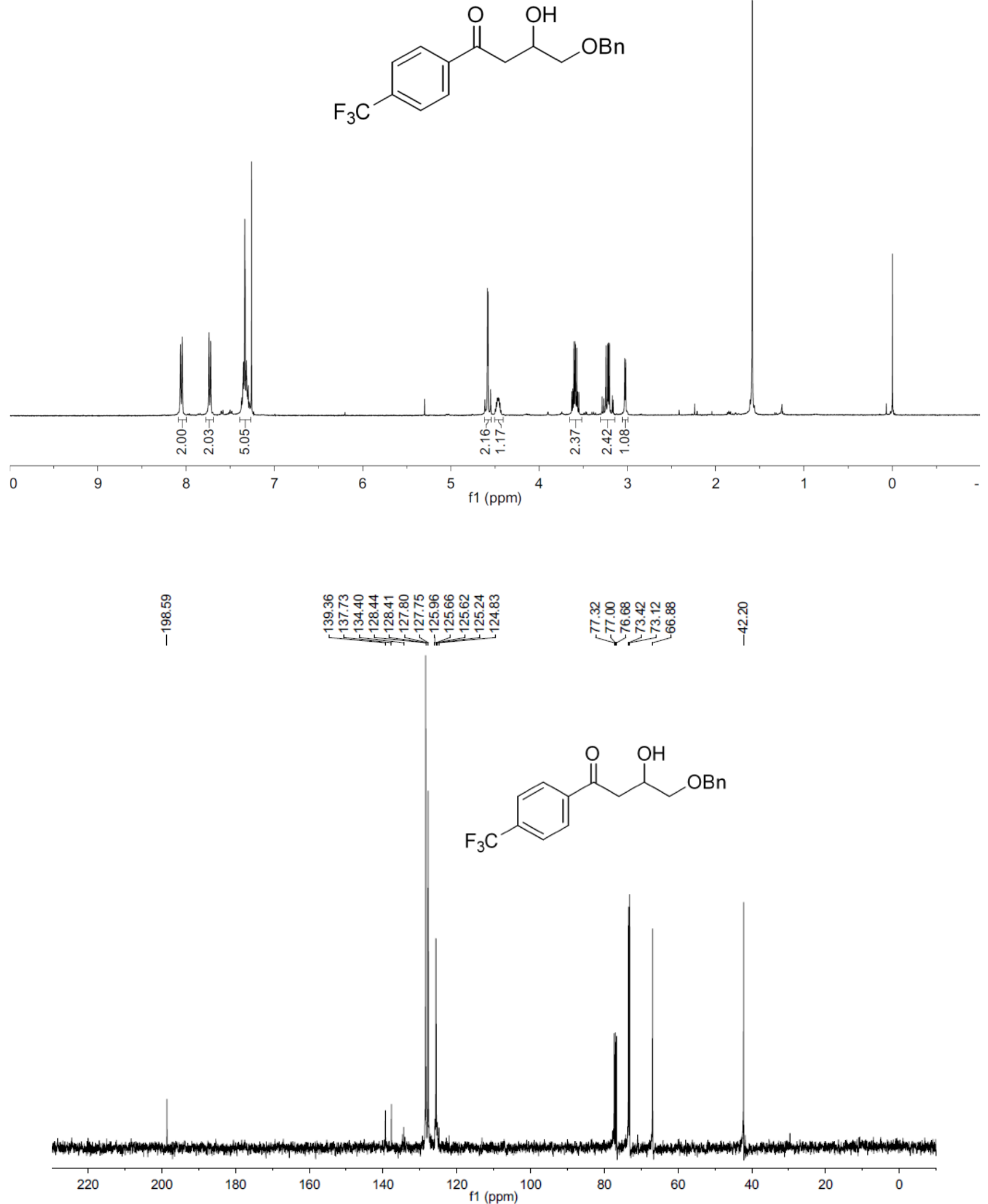


\section{$2 \mathrm{gg}$}

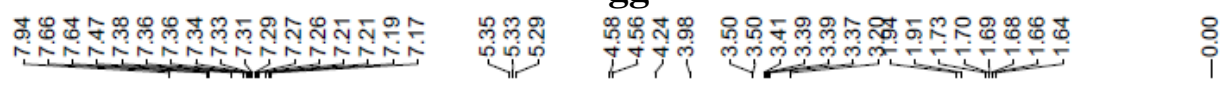
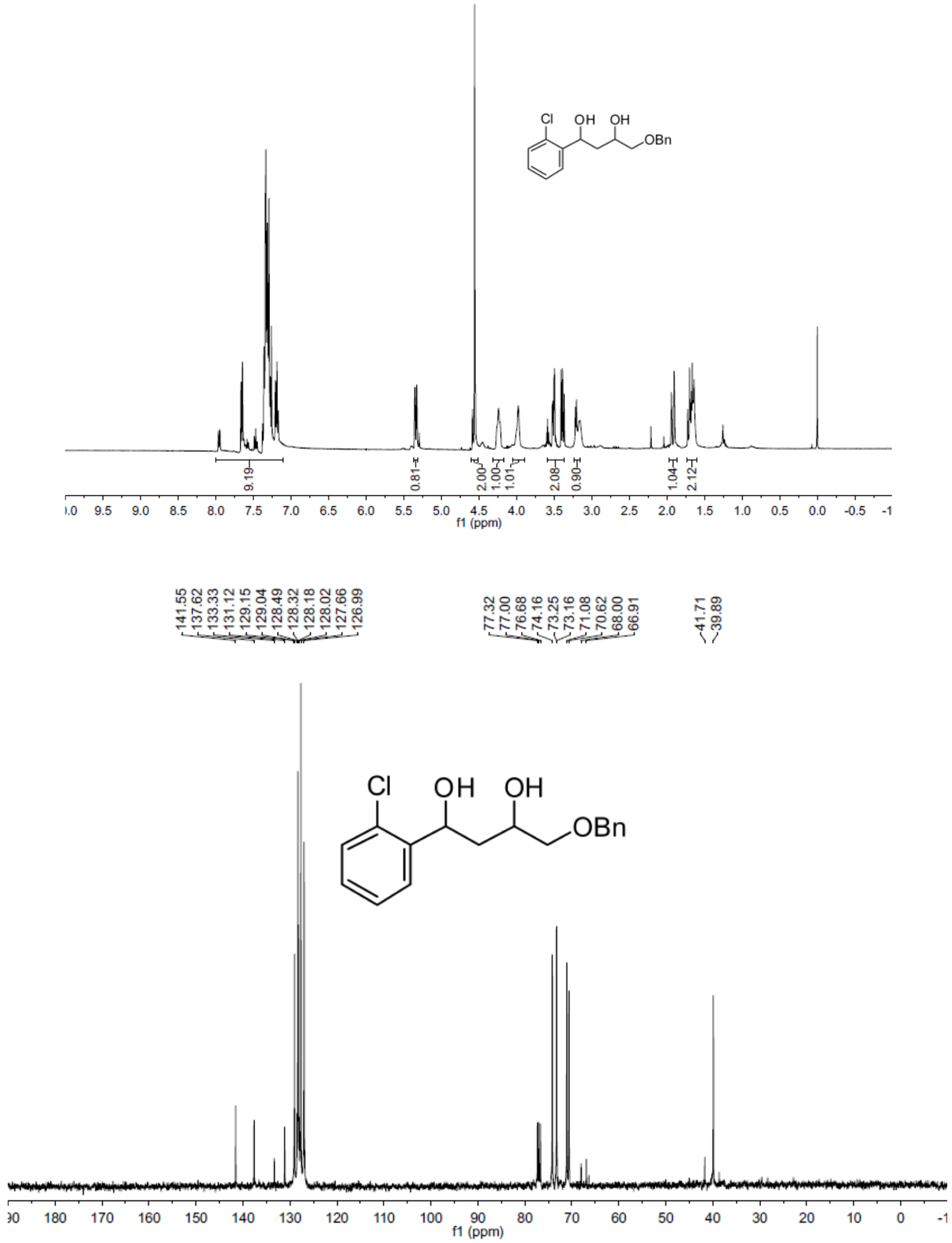

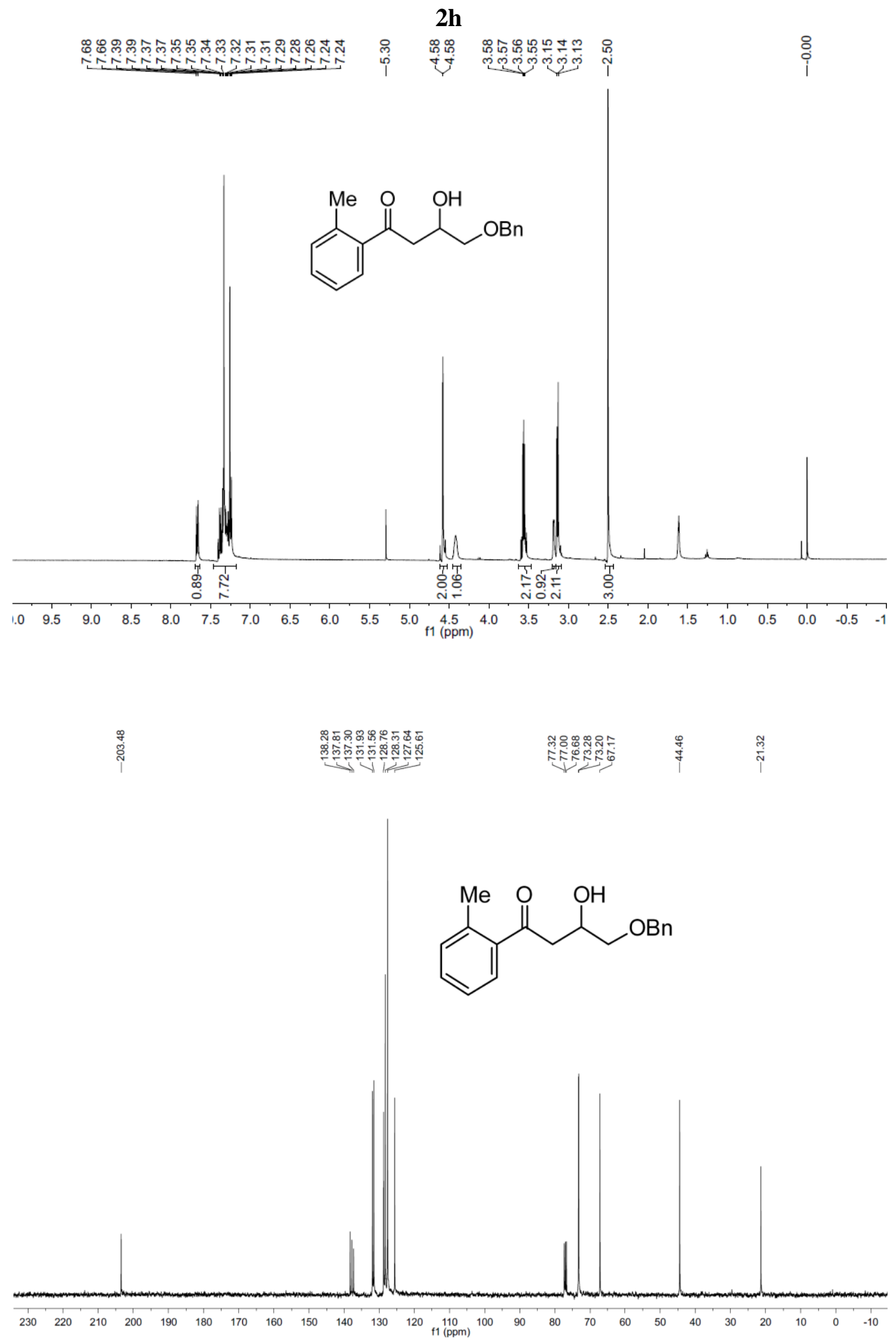


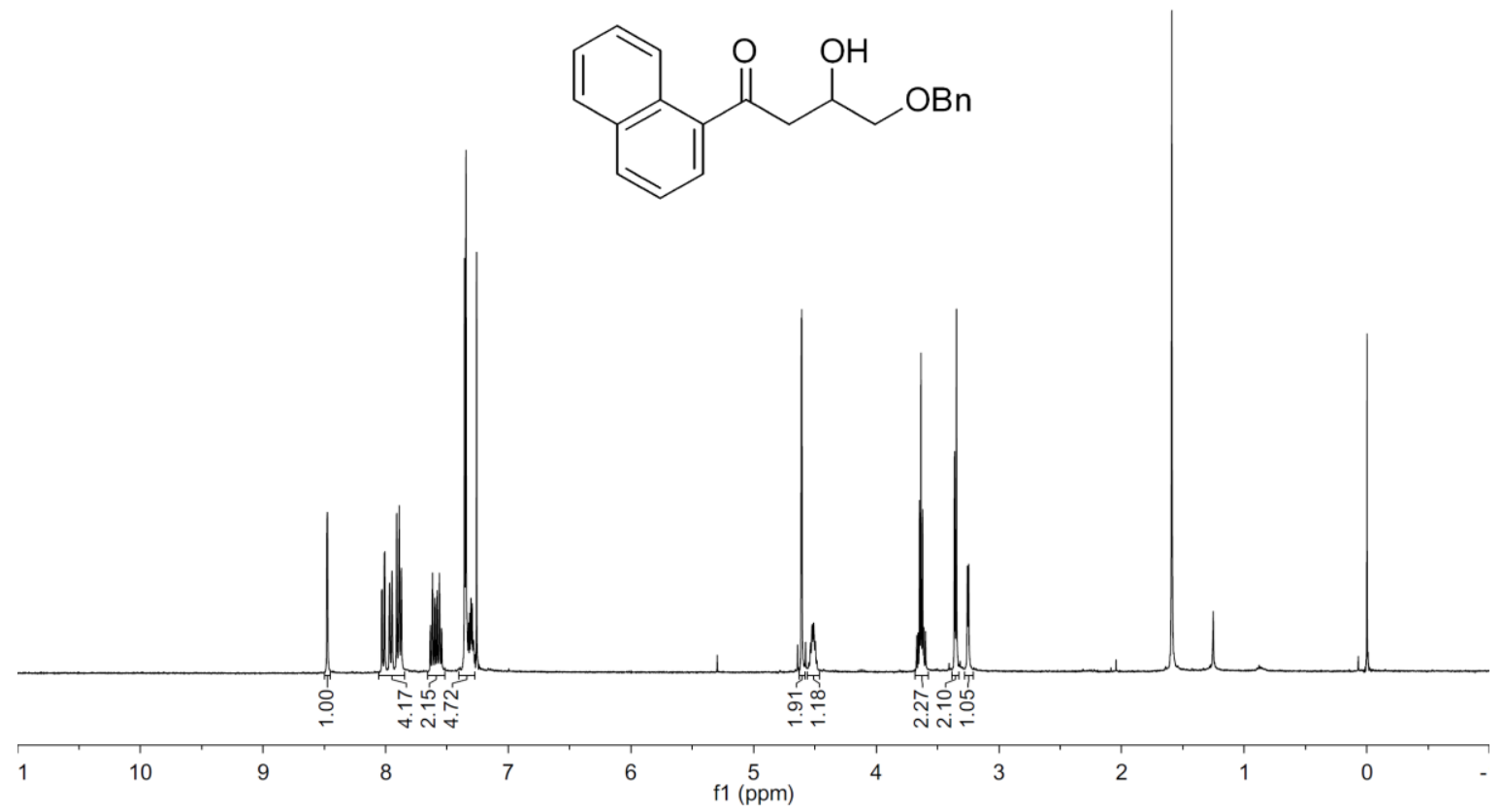

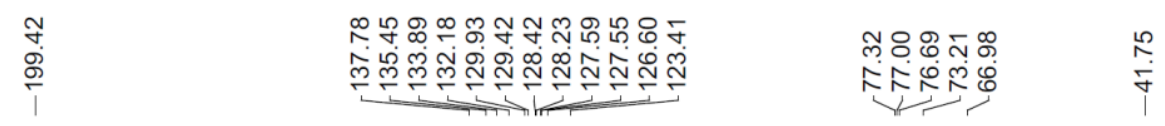

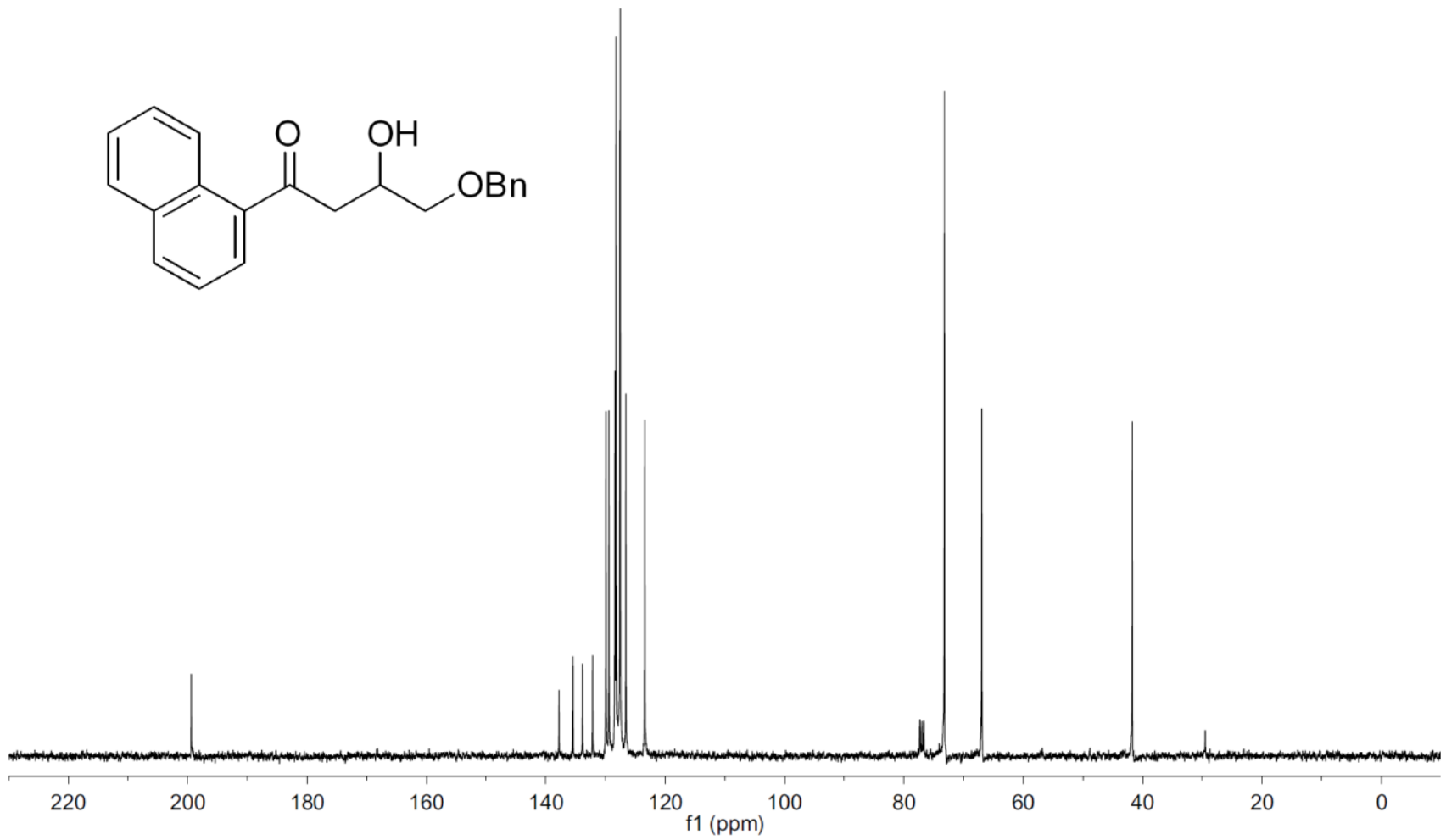




\section{$2 \mathbf{j}$}

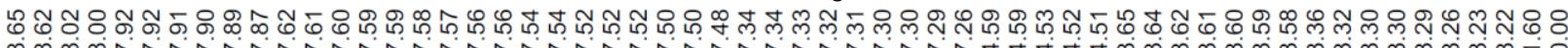

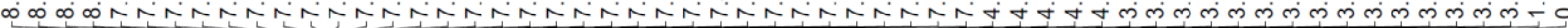

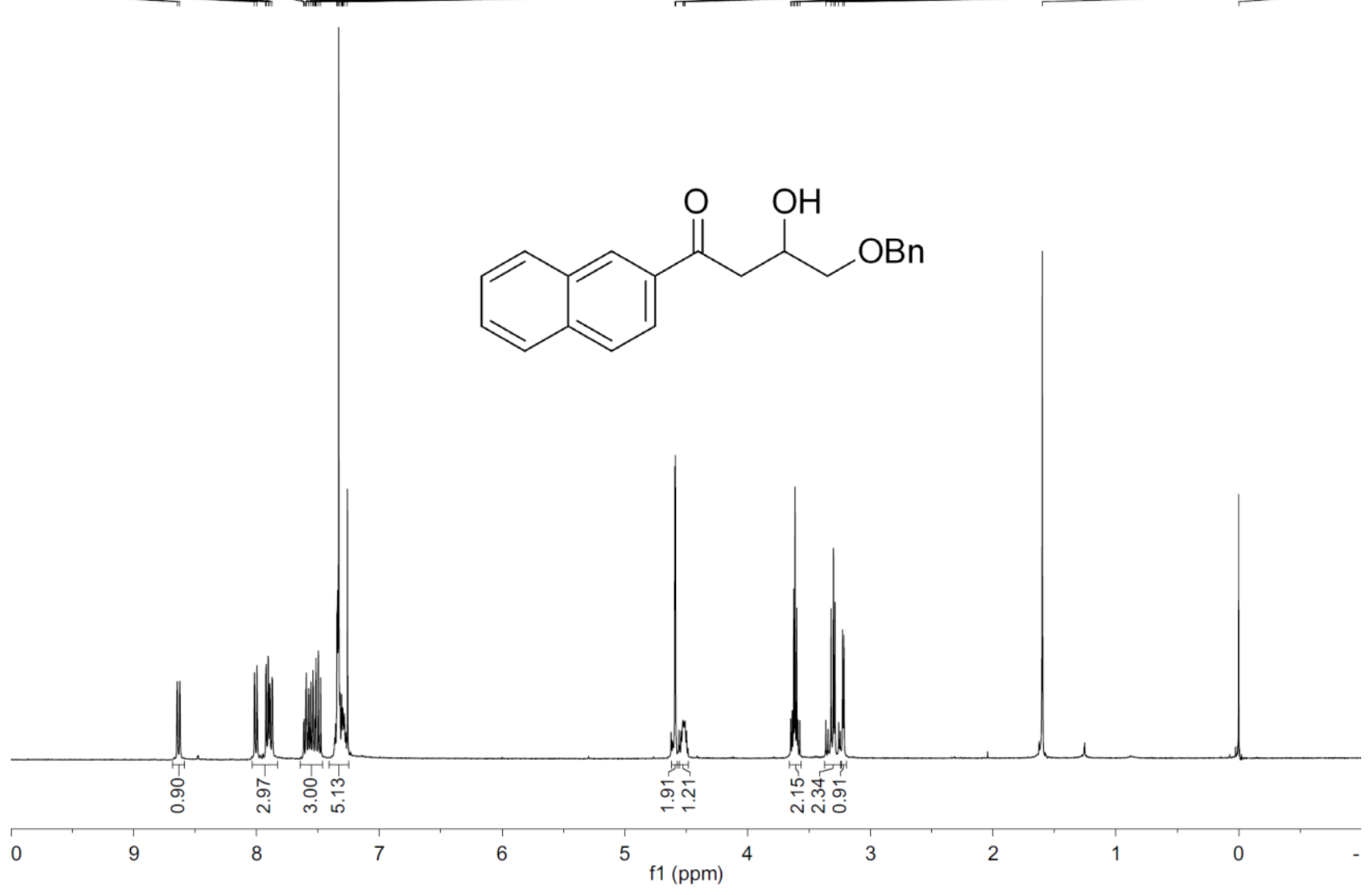

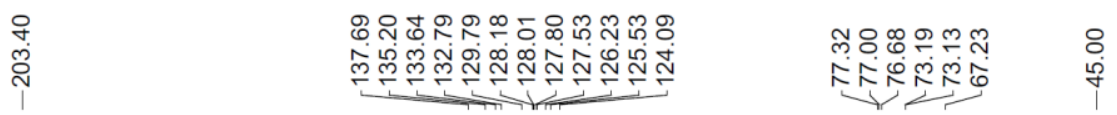

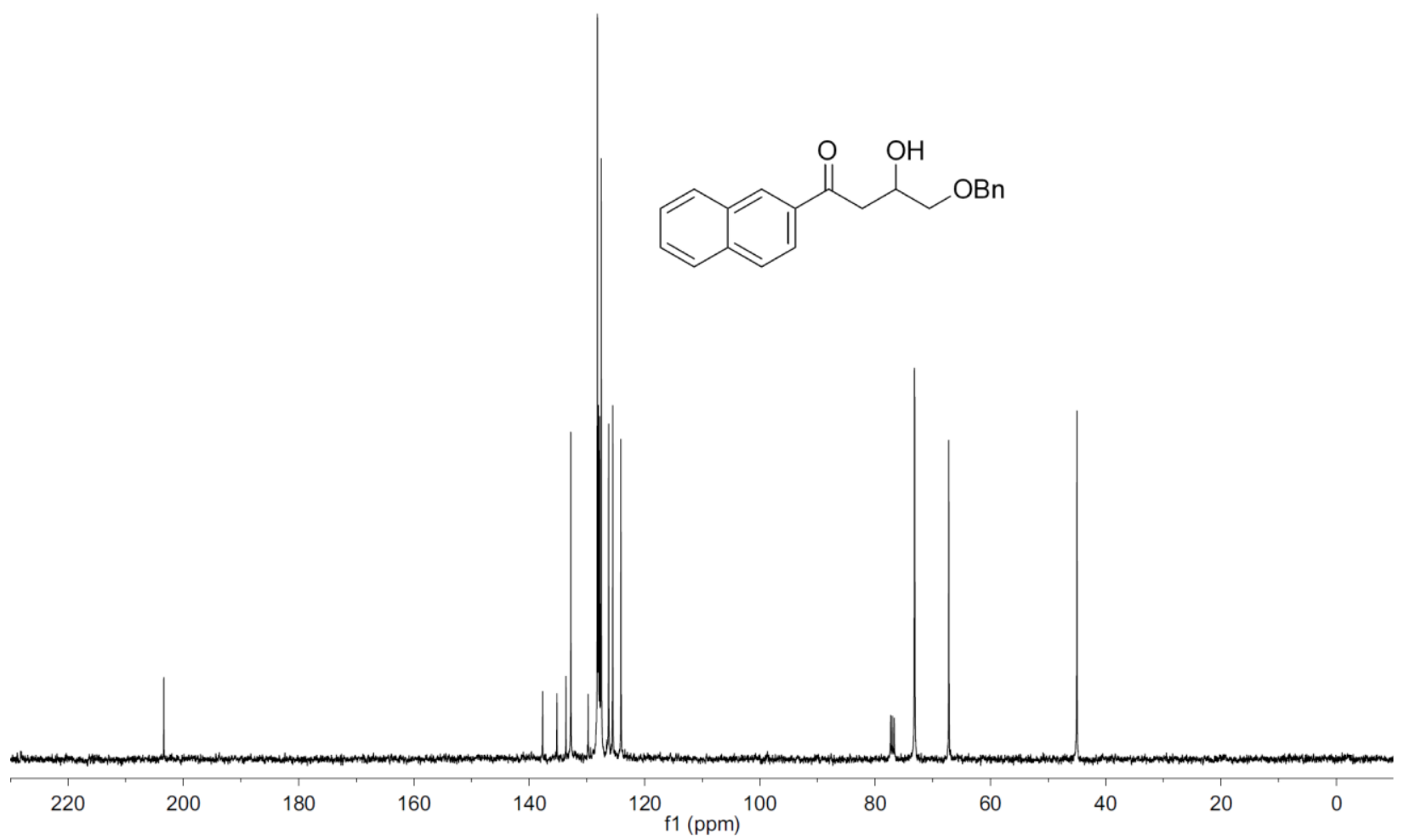


2k

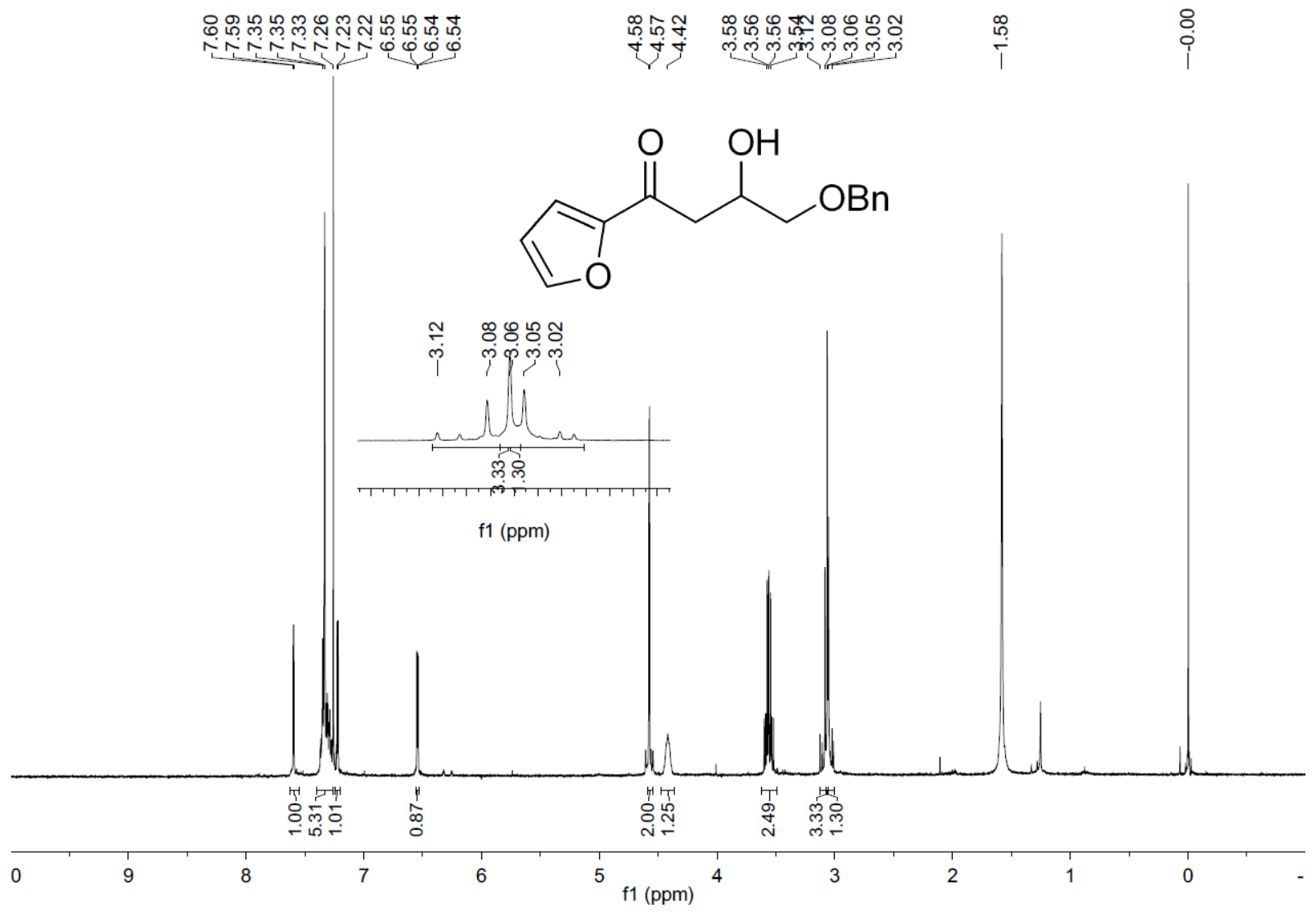

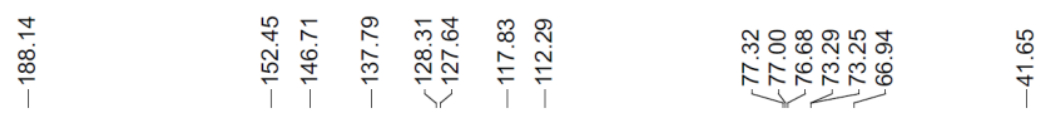

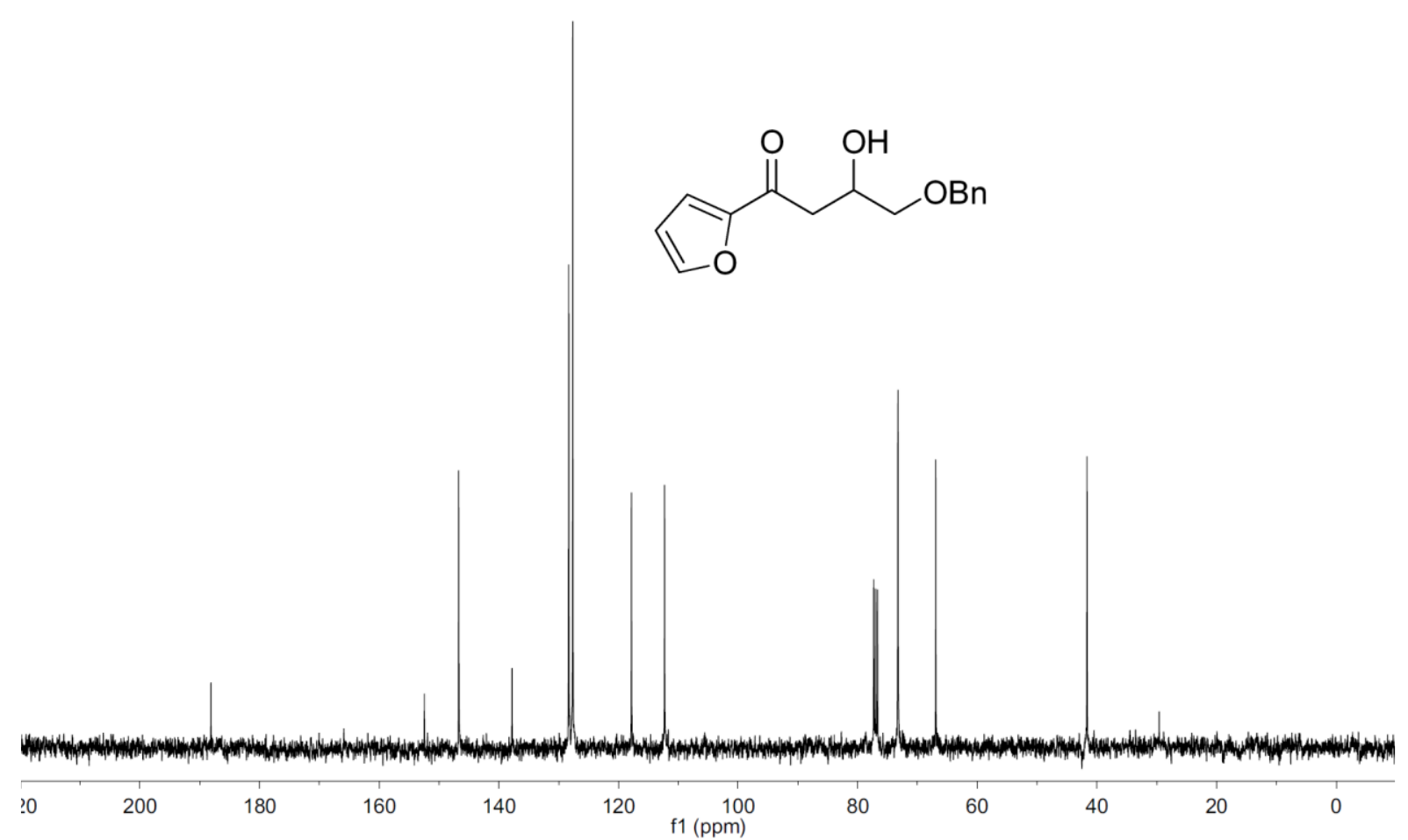




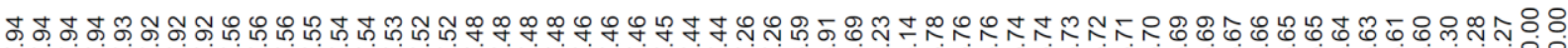

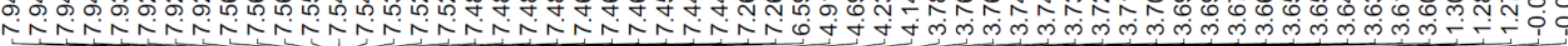<smiles>CCOC(OCC)C(=O)CC(=O)c1ccccc1</smiles>

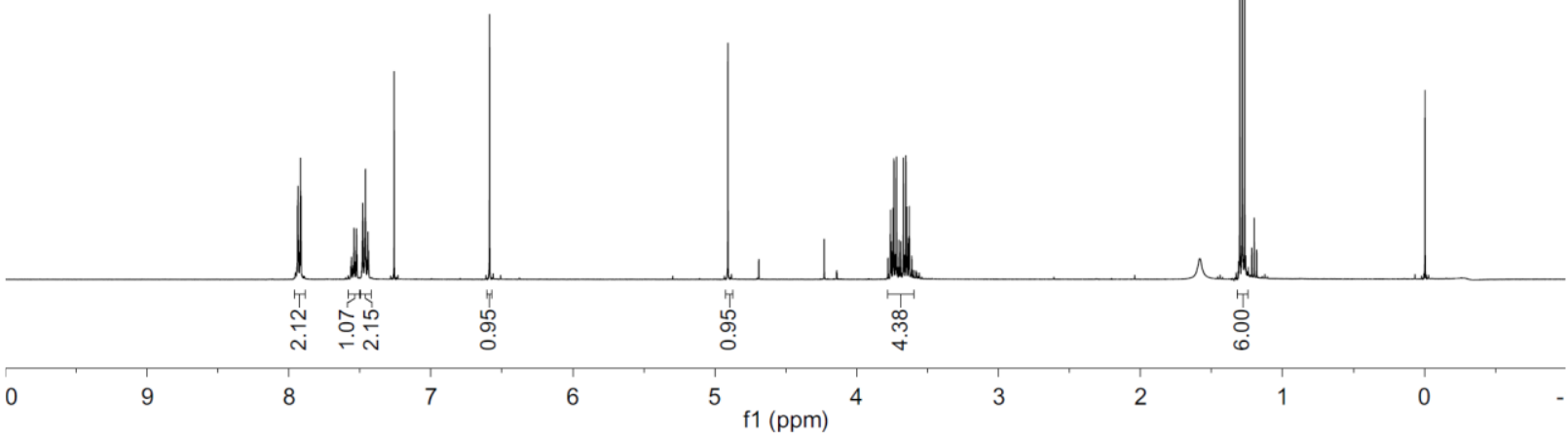
รั. รั่
누욤눈
लำ ్ㅠㄴ
\%

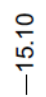<smiles>CCOC(OCC)C(=O)CC(=O)c1ccccc1</smiles> 
3b

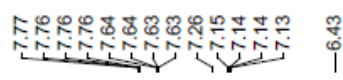

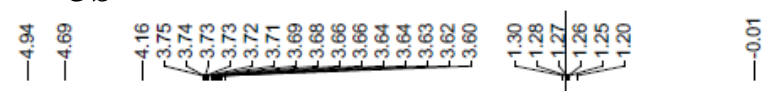

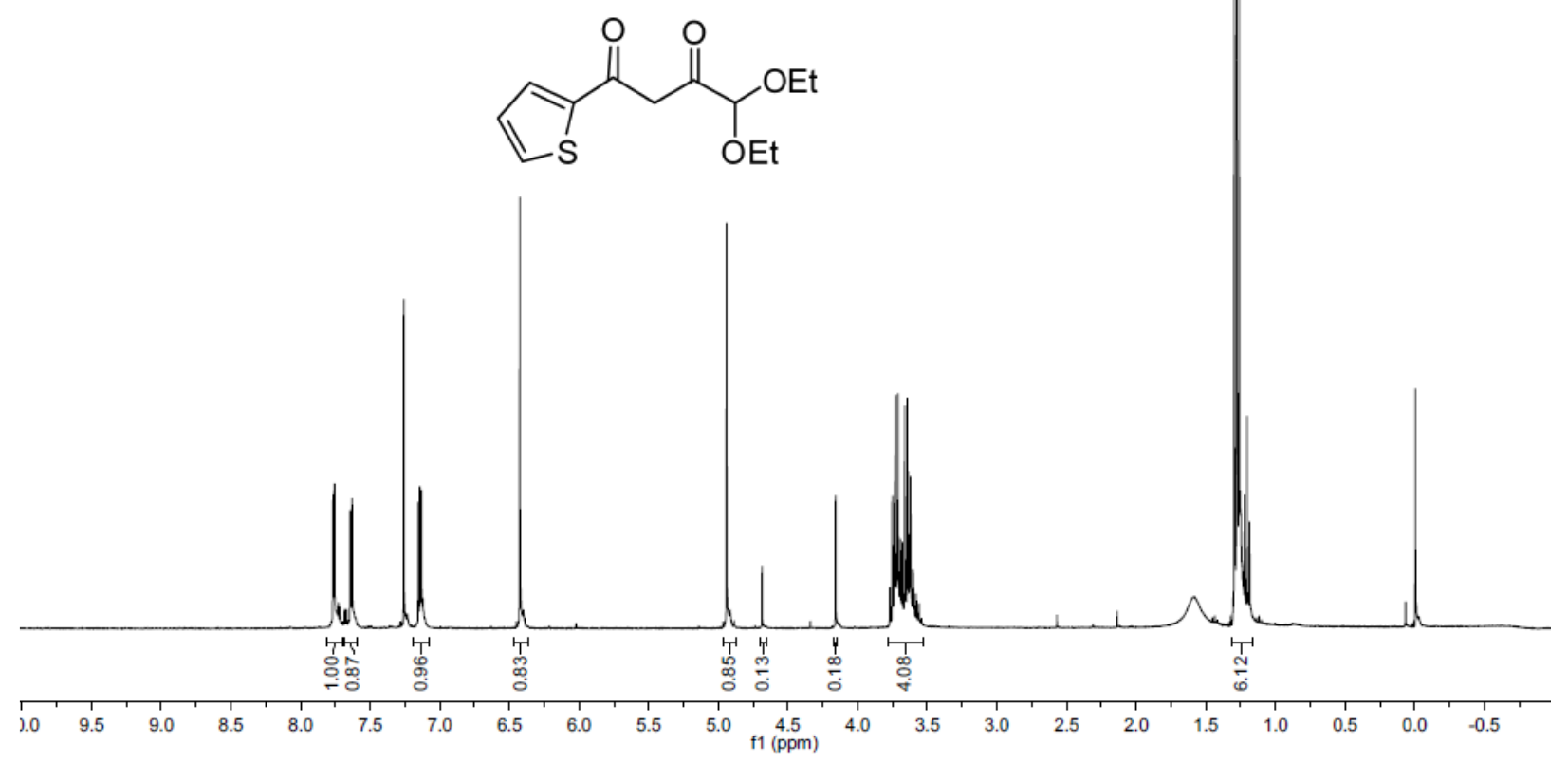

每<smiles>CCOC(OCC)C(=O)CC(=O)c1cccs1</smiles>

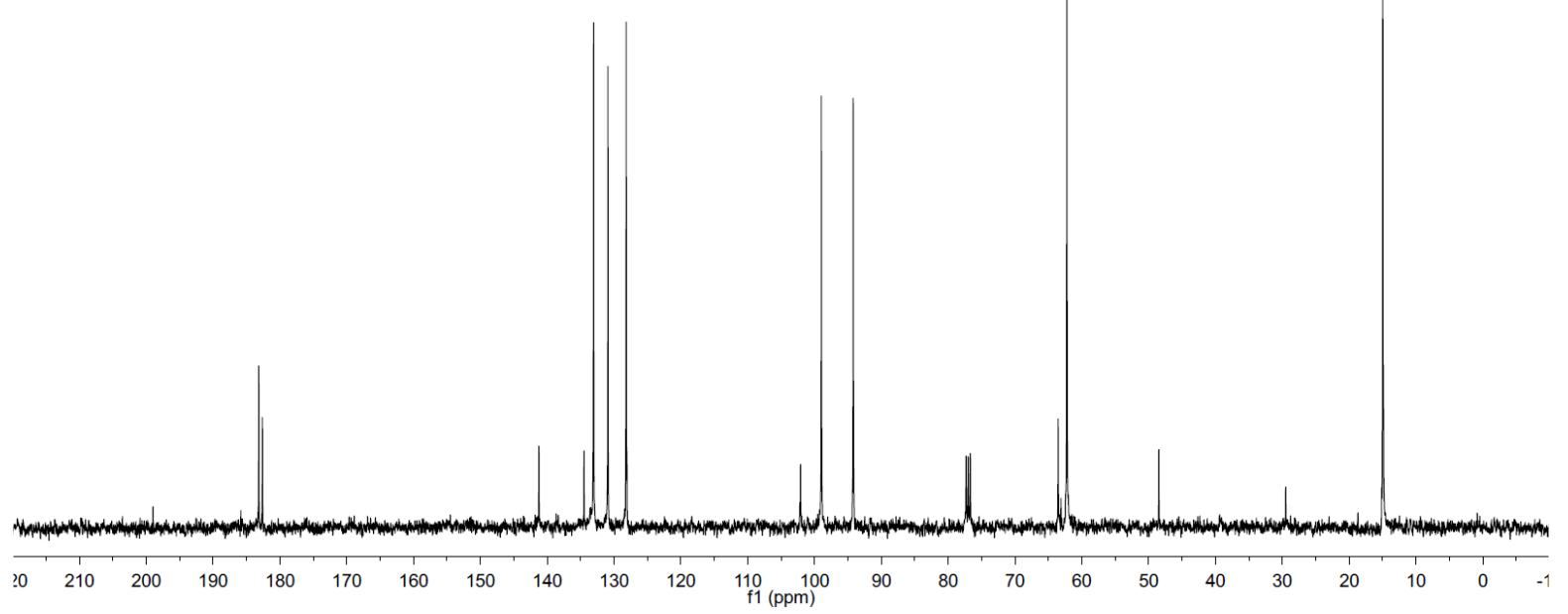



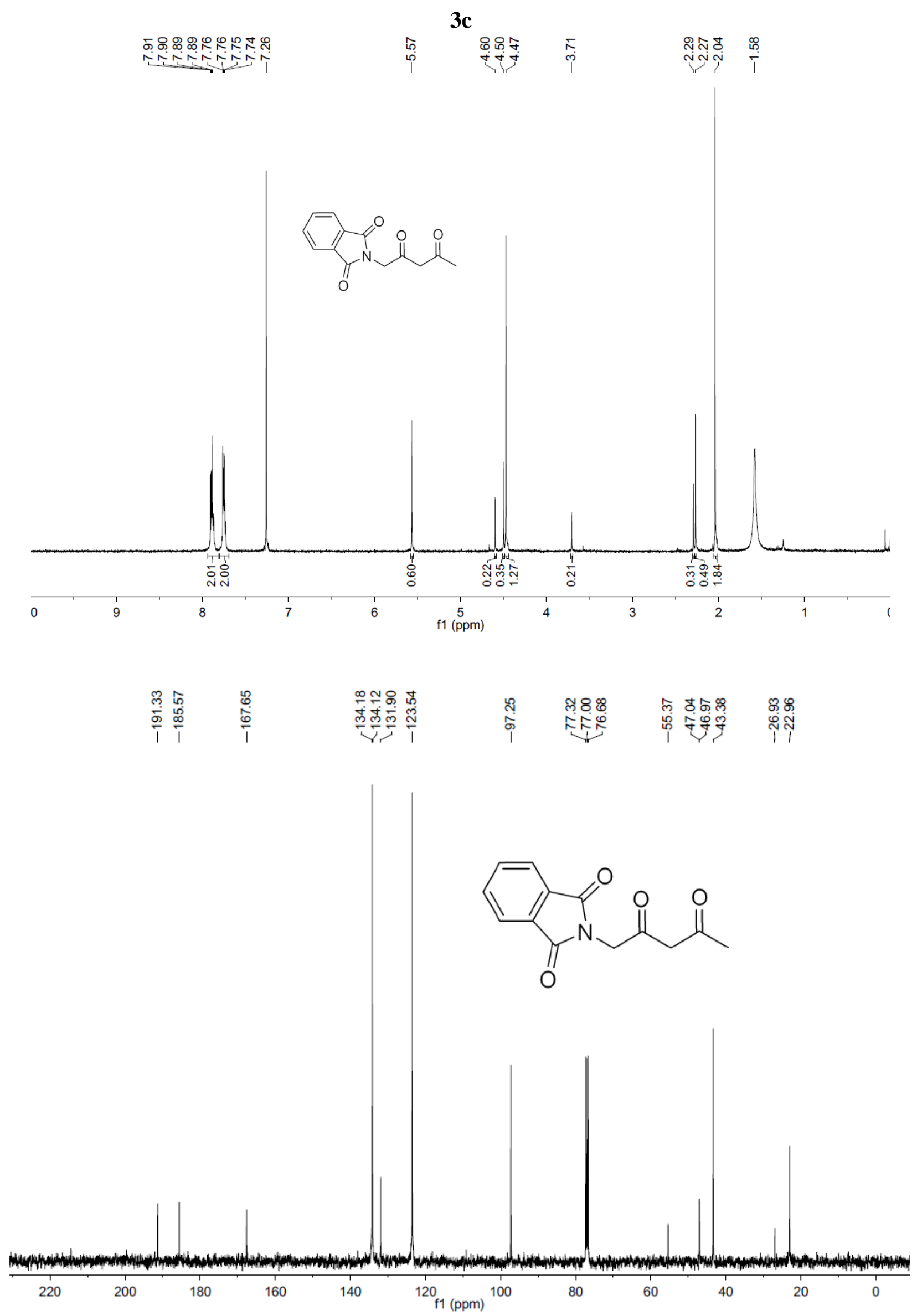


\section{3d}

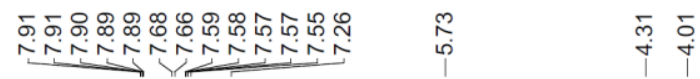

꾹워

¿’<smiles></smiles>

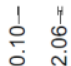

○ั

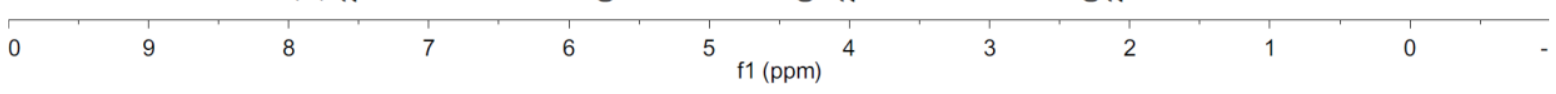

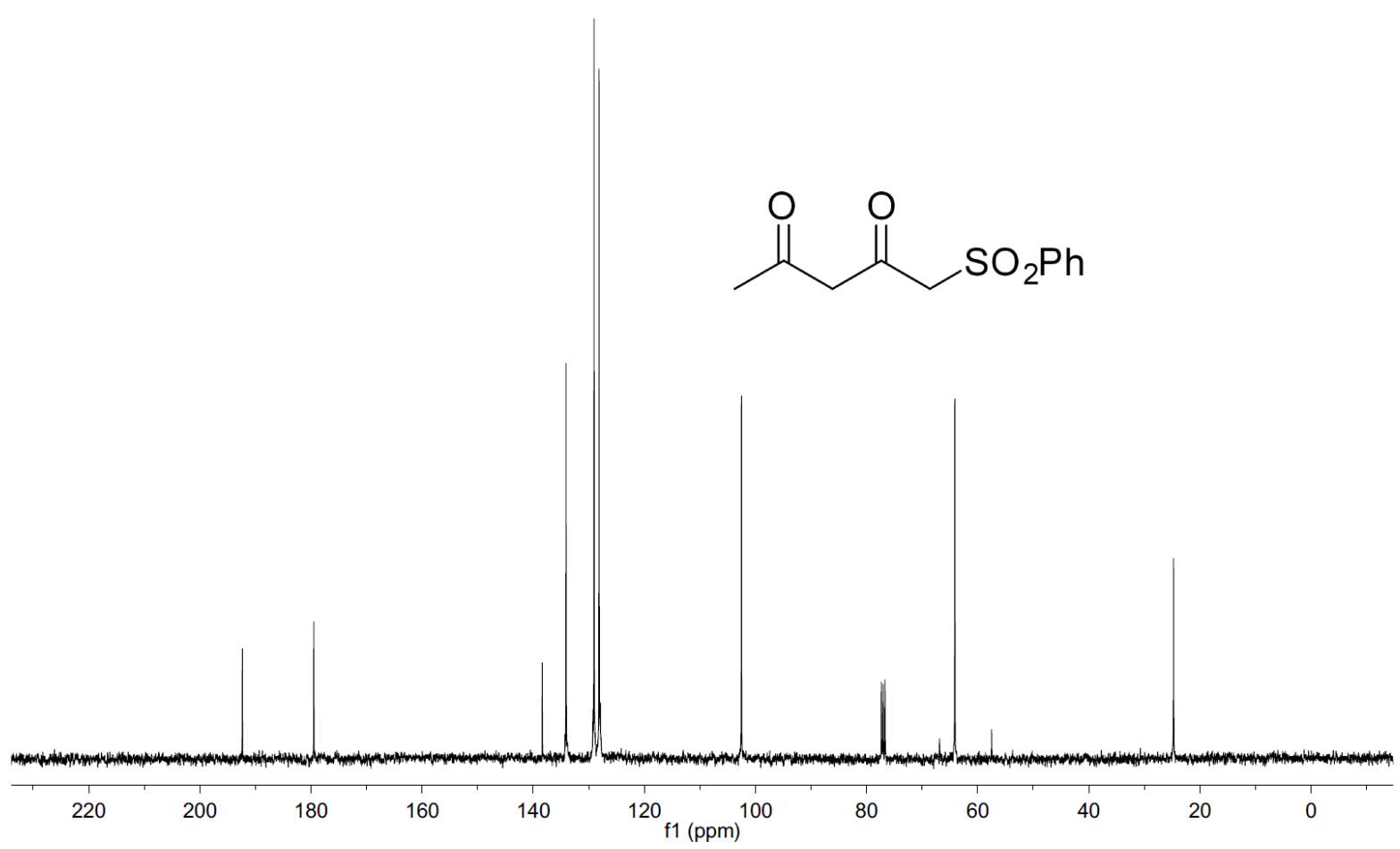


$3 e$

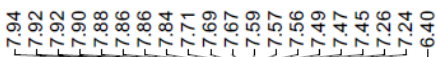

鱼过

$\stackrel{\circ}{i}$

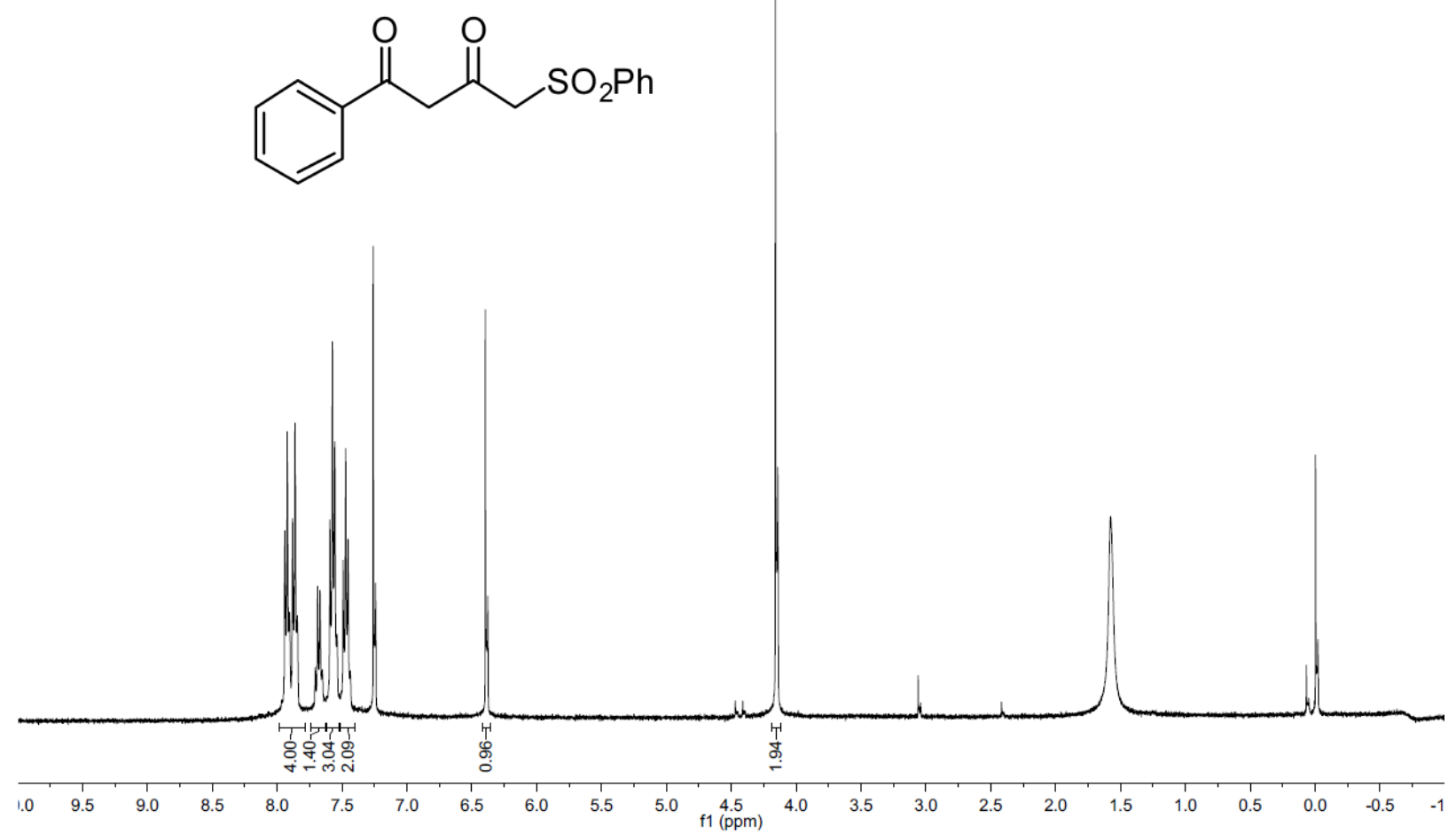

ำ

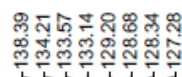

$\begin{array}{ll}8 & 8 \\ 0 & 0 \\ 1 & 10\end{array}$

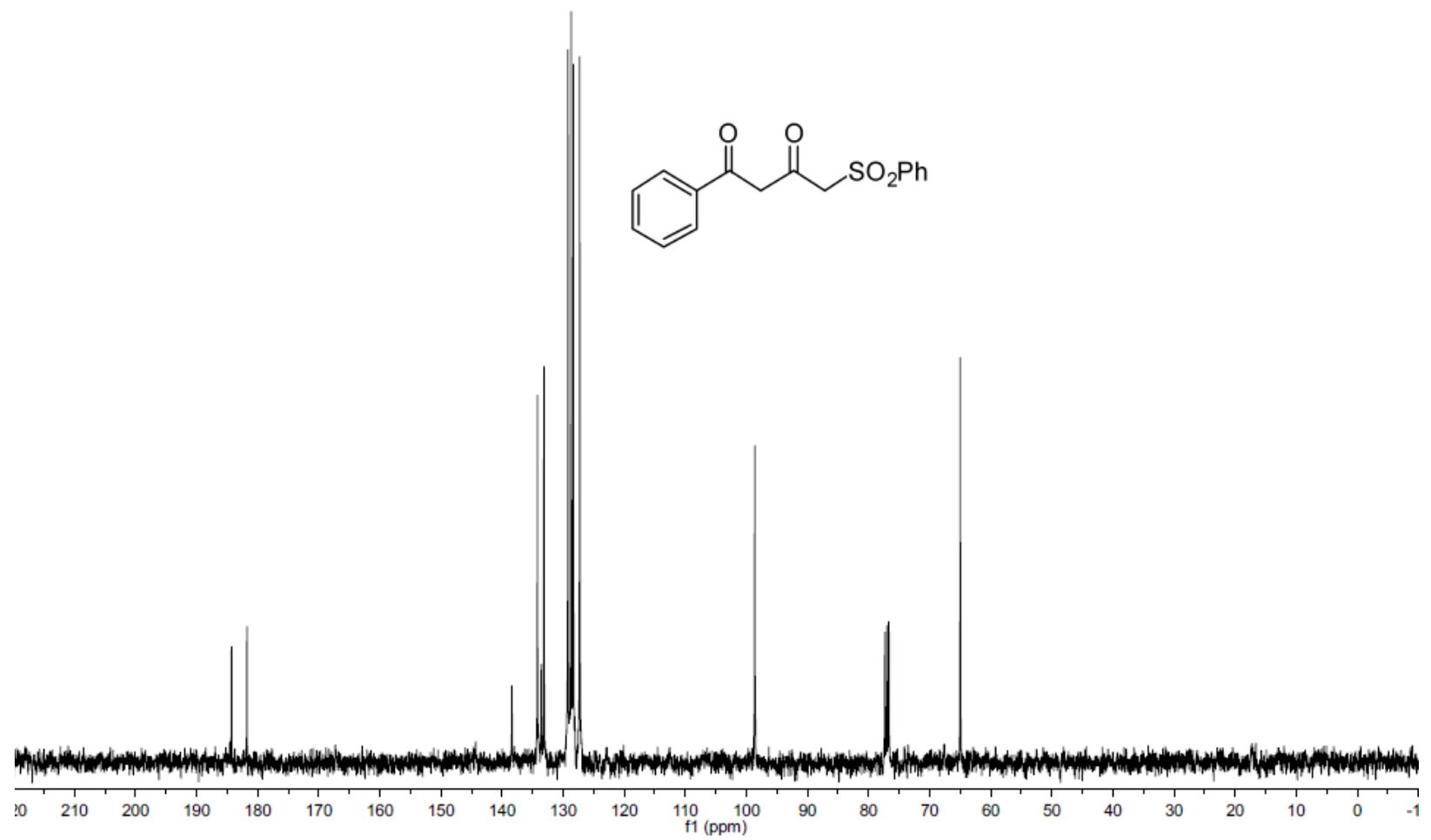




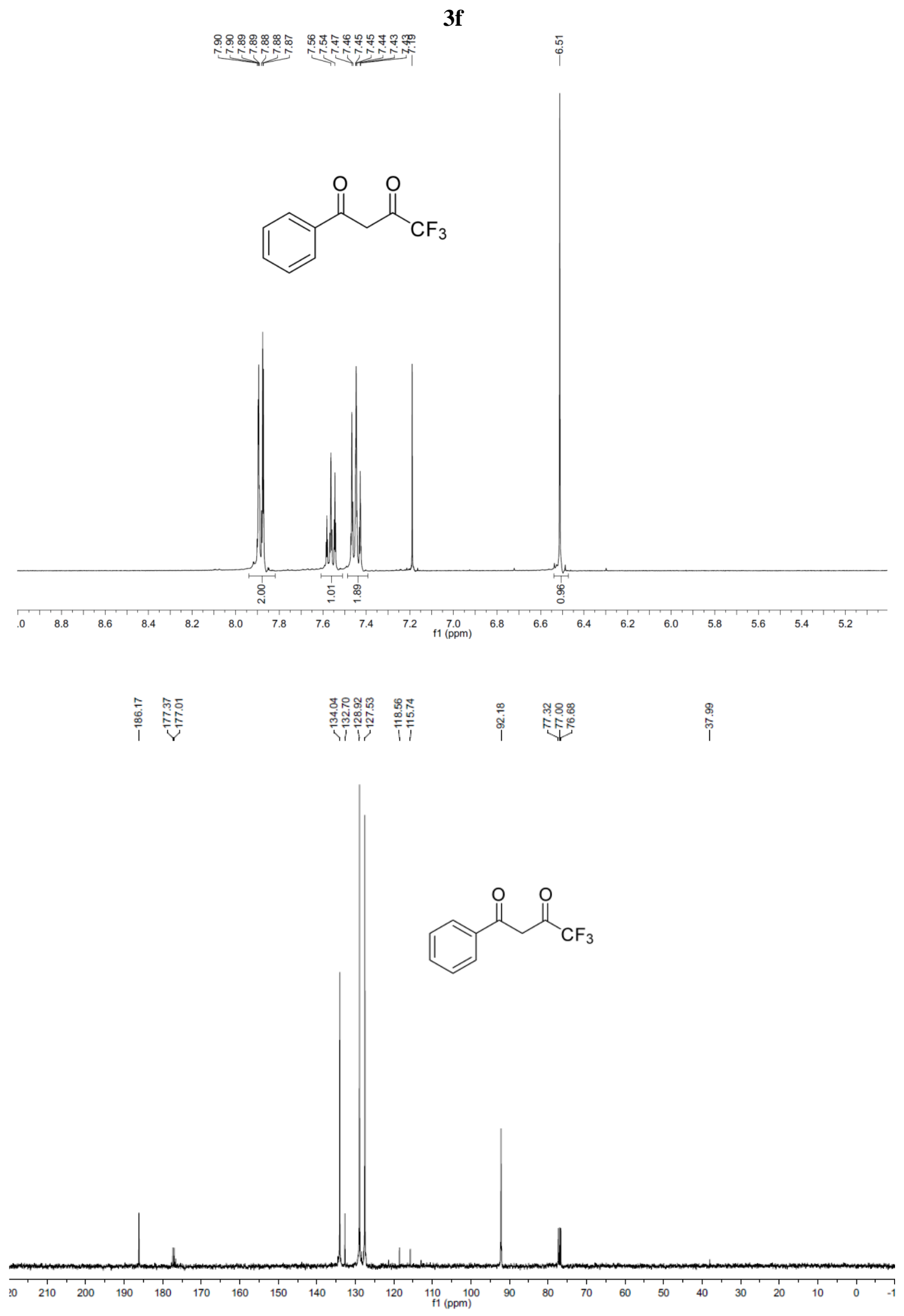



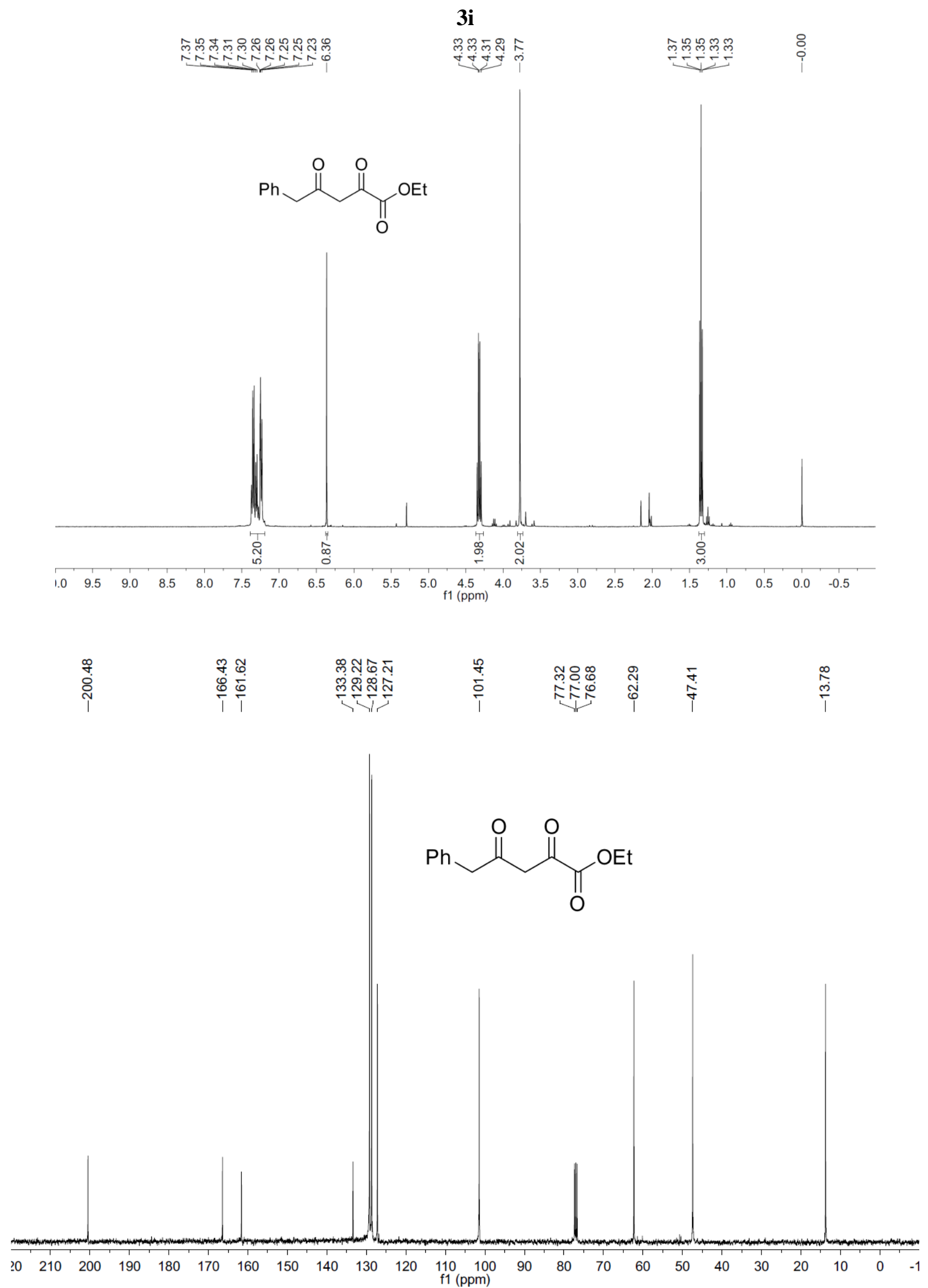


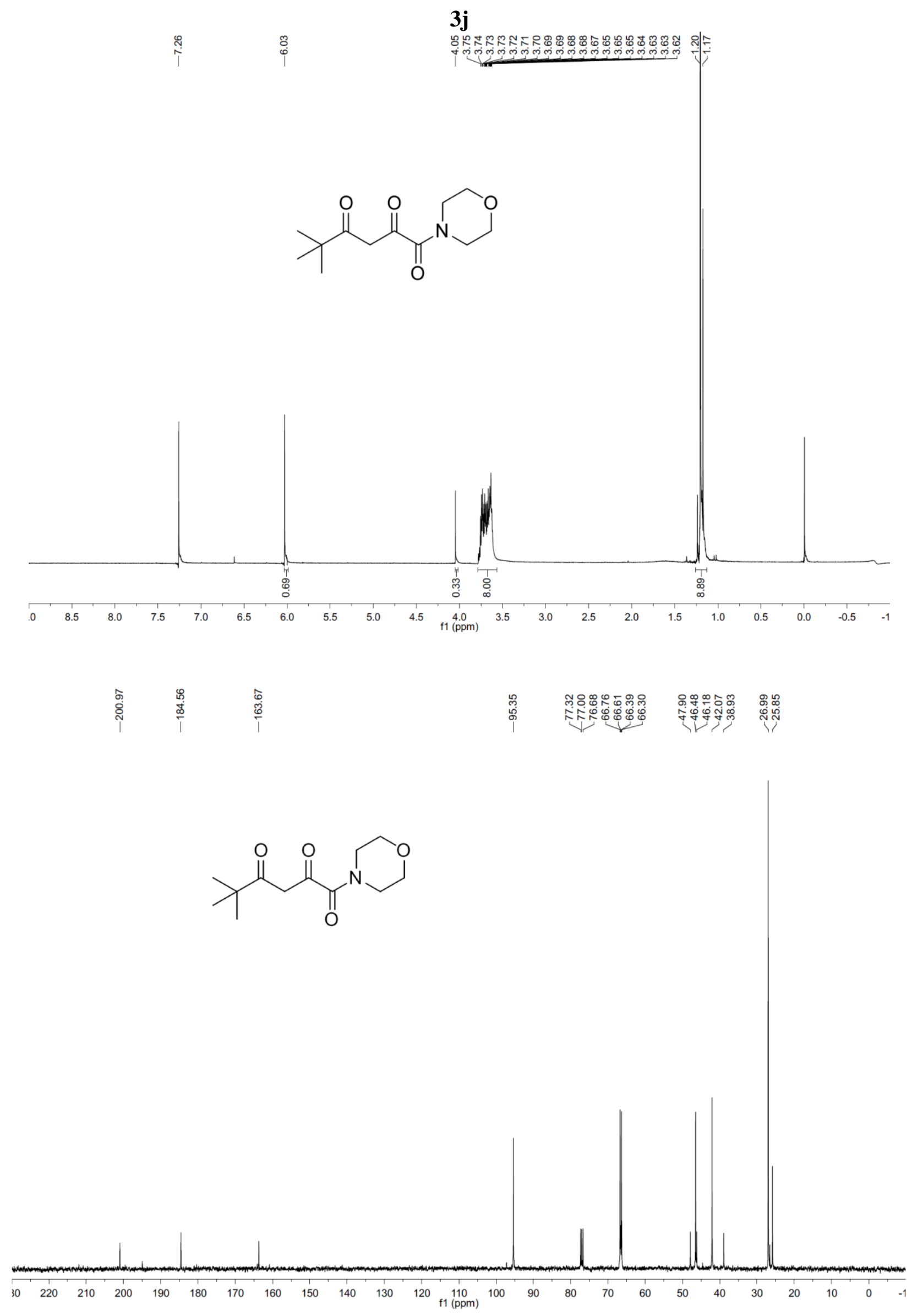


$4 a$

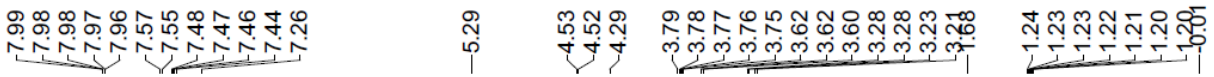<smiles>CCOC(OCC)C(O)CC(=O)c1ccccc1</smiles>

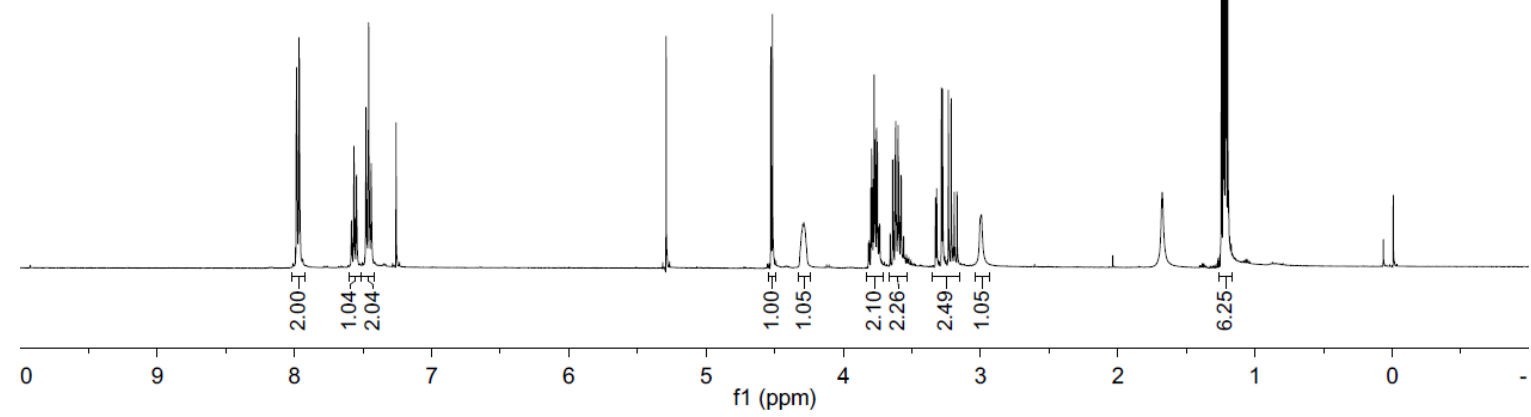

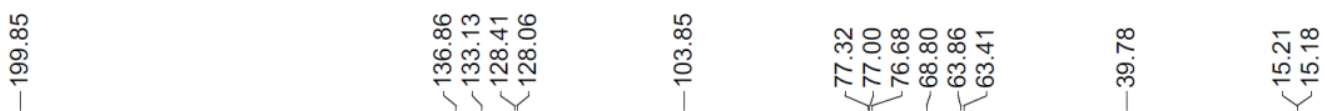

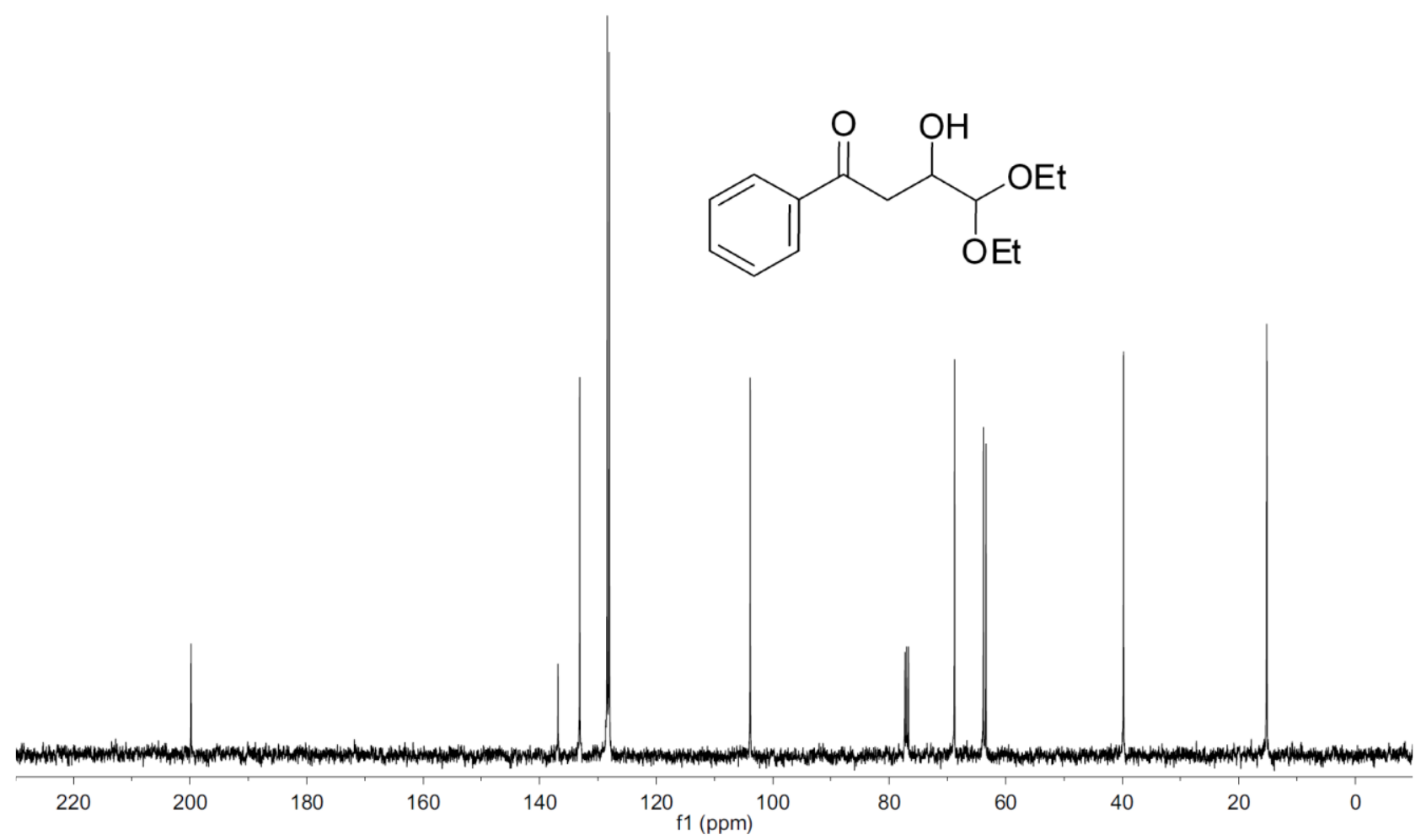


4b

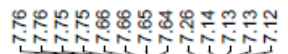

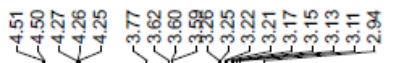

$\sqrt[3]{\frac{9}{y}}$

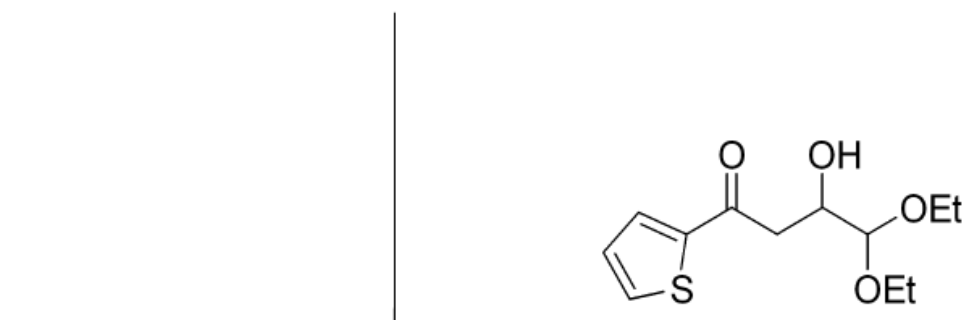

Et

$$
\text { . }
$$

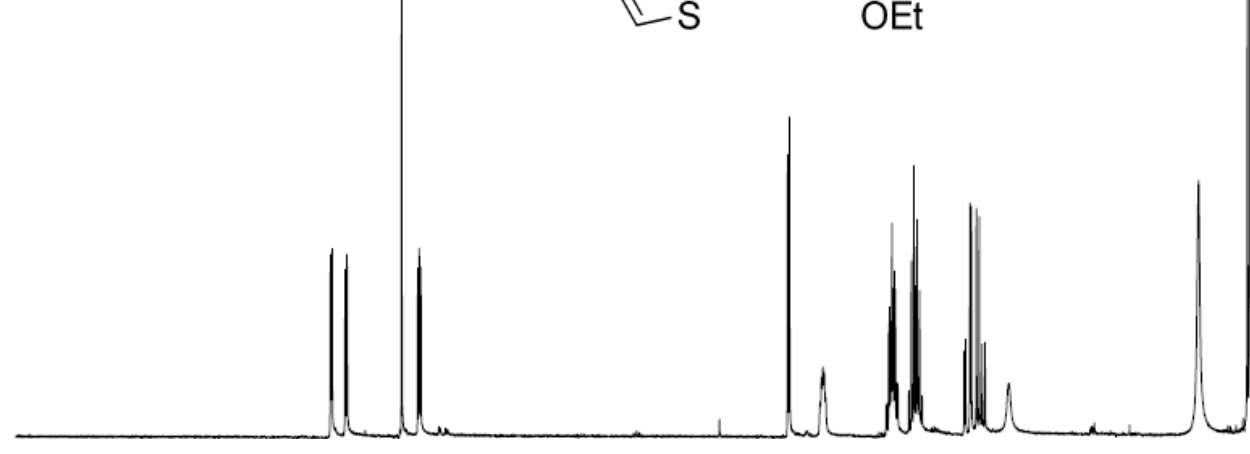

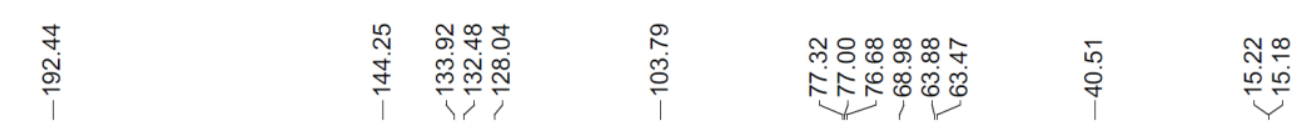<smiles>CCOC(OCC)C(O)CC(=O)c1cccs1</smiles>

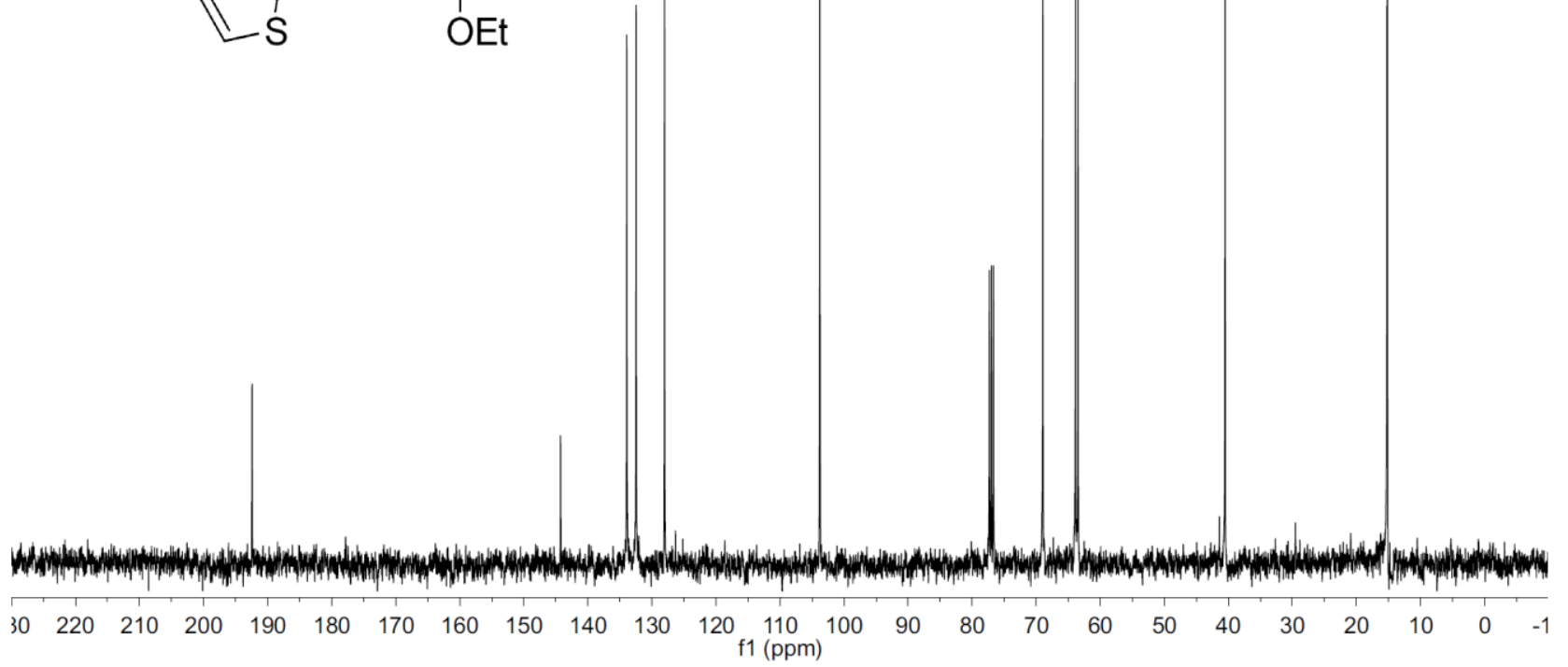



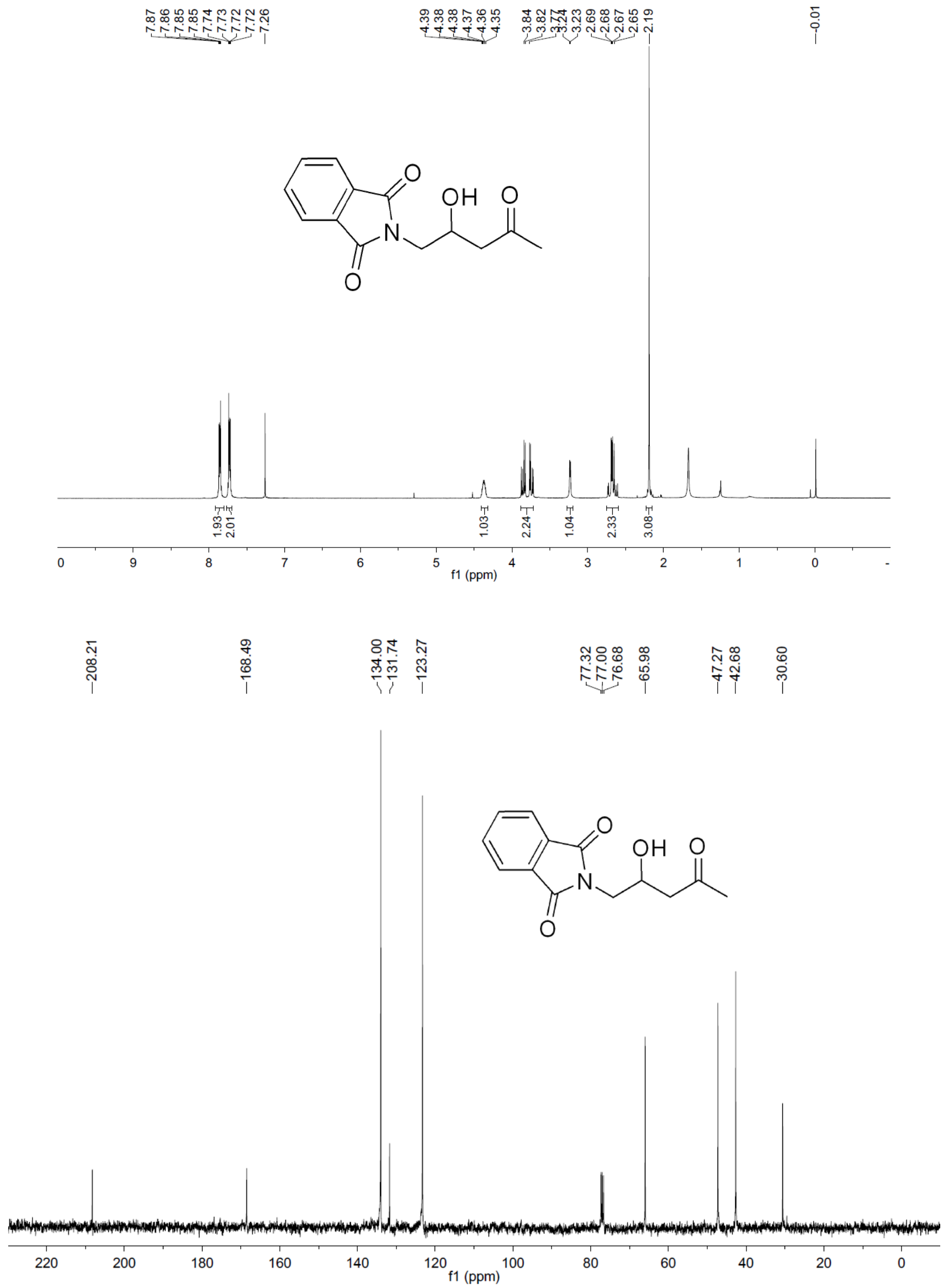


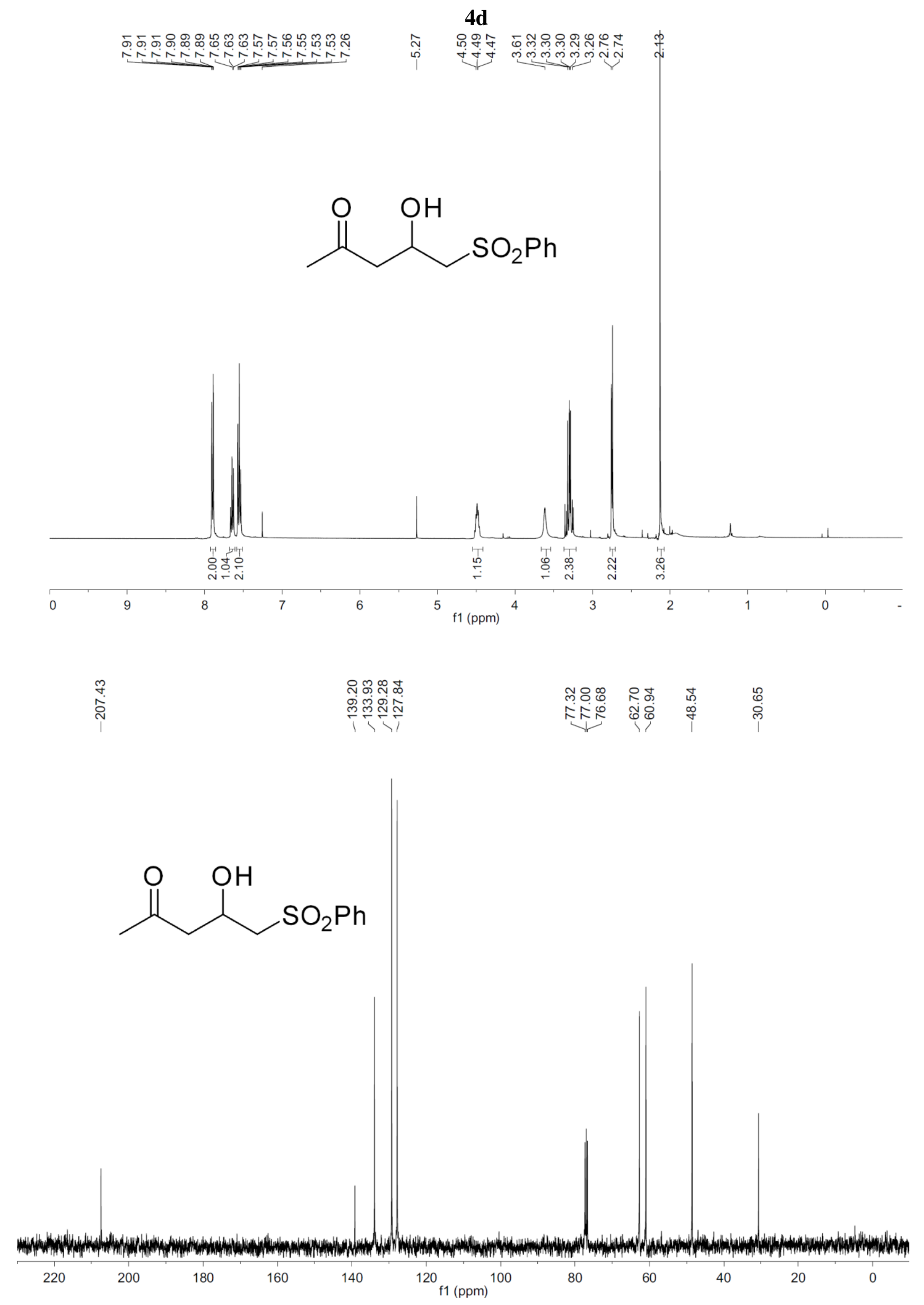



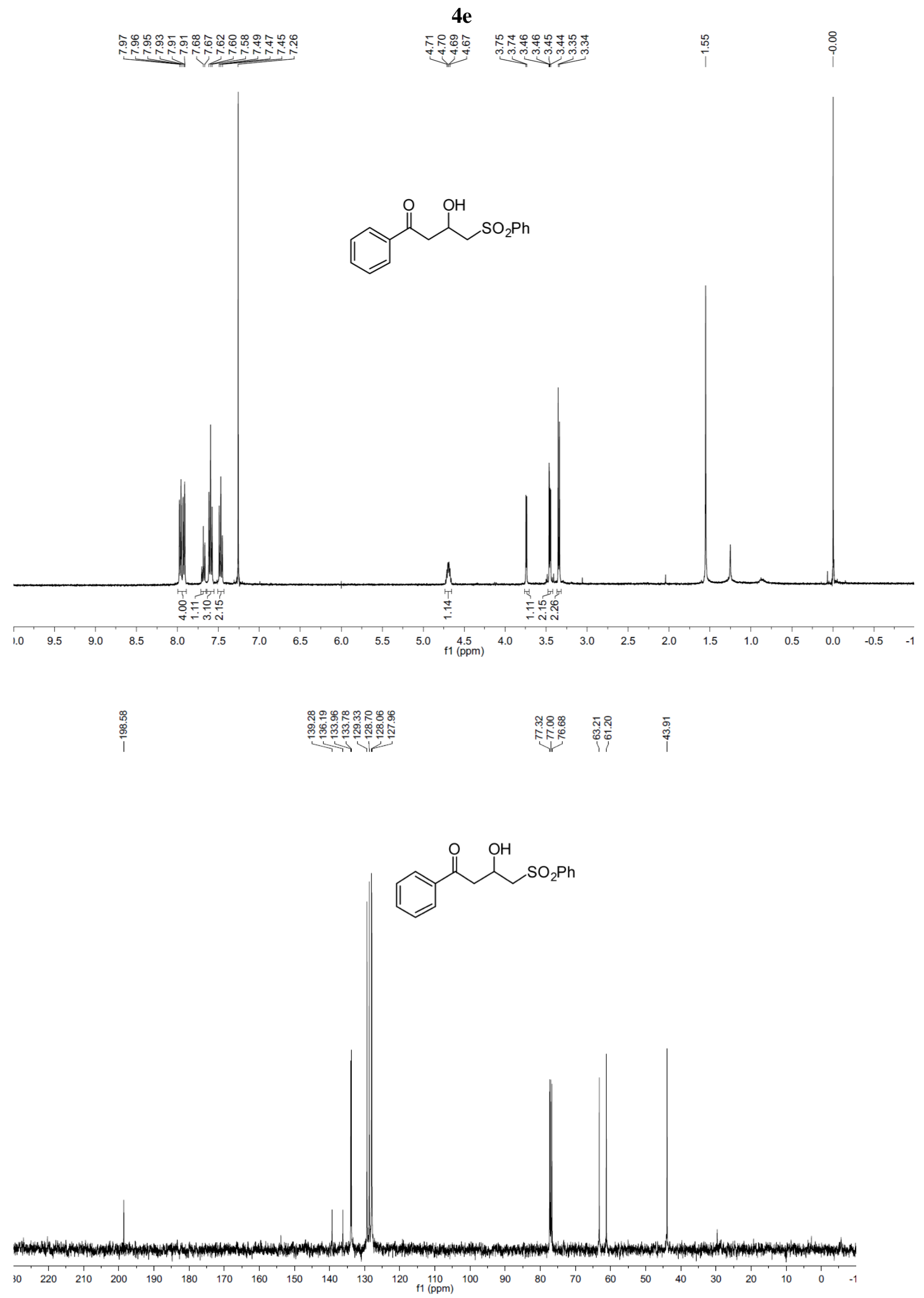

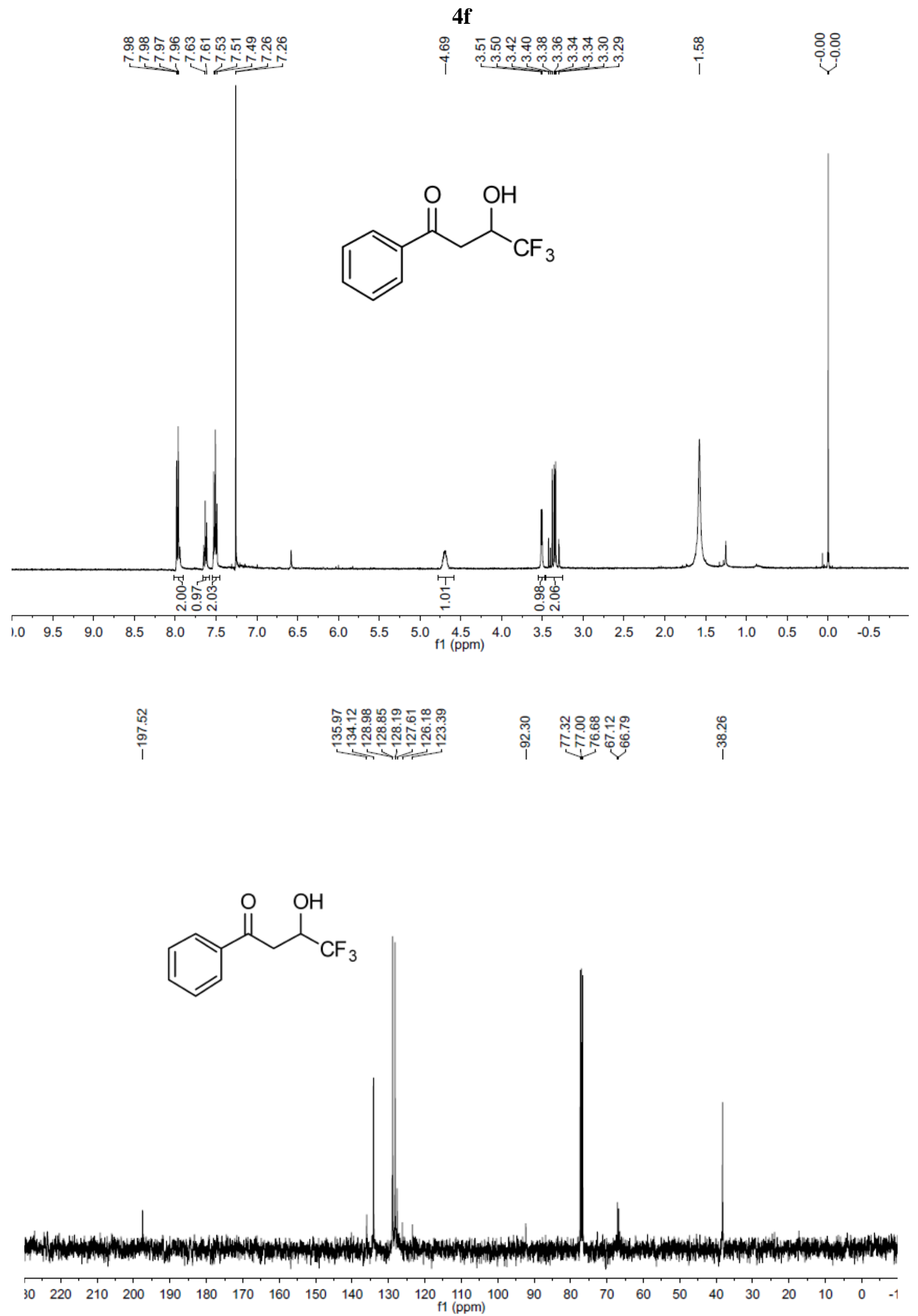

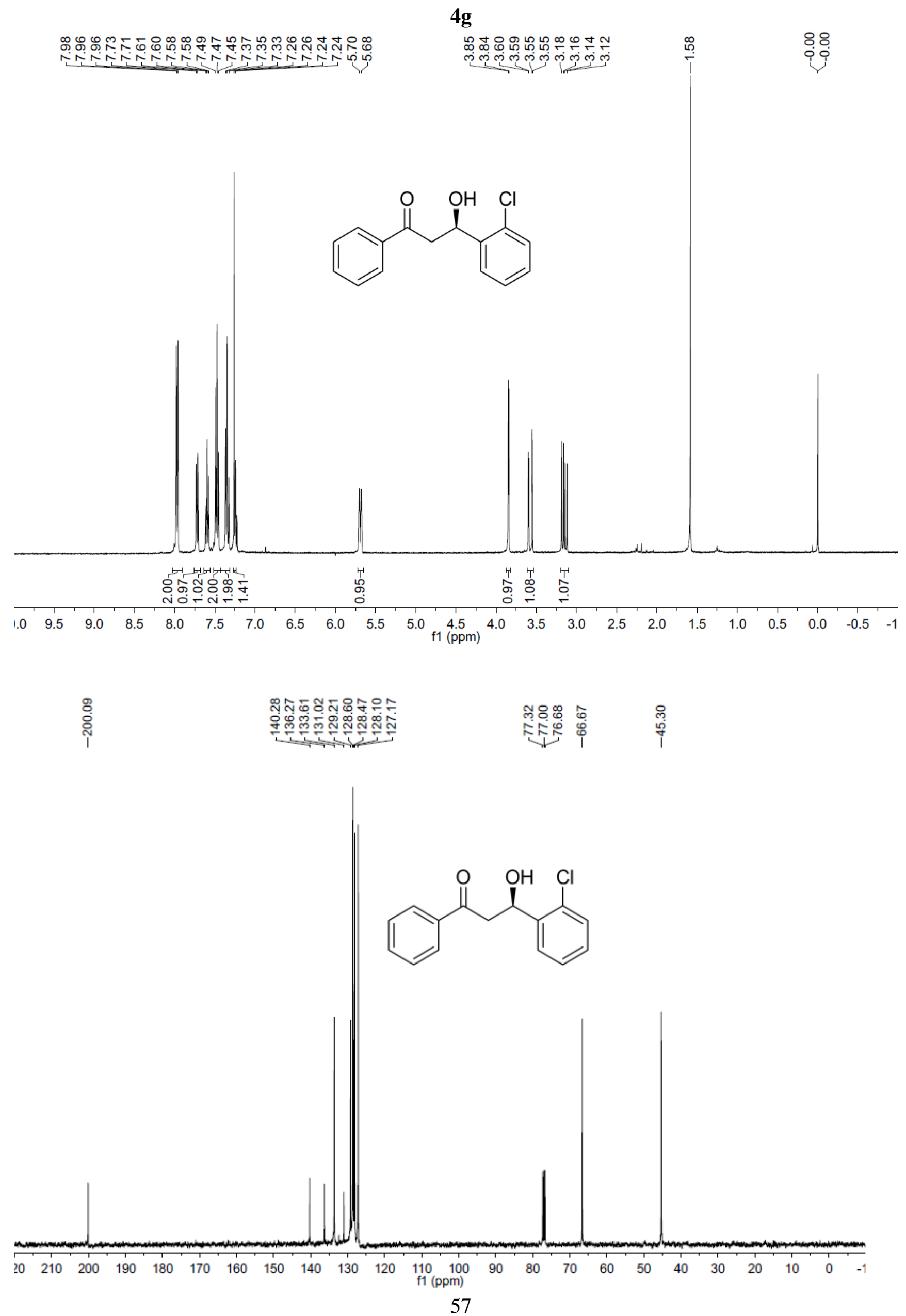


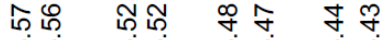

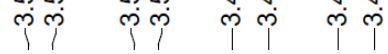

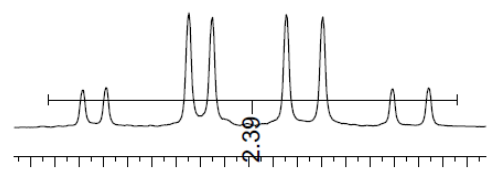

f1 (ppm)<smiles>CCOC(=O)C(O)CC(=O)c1ccccc1</smiles>

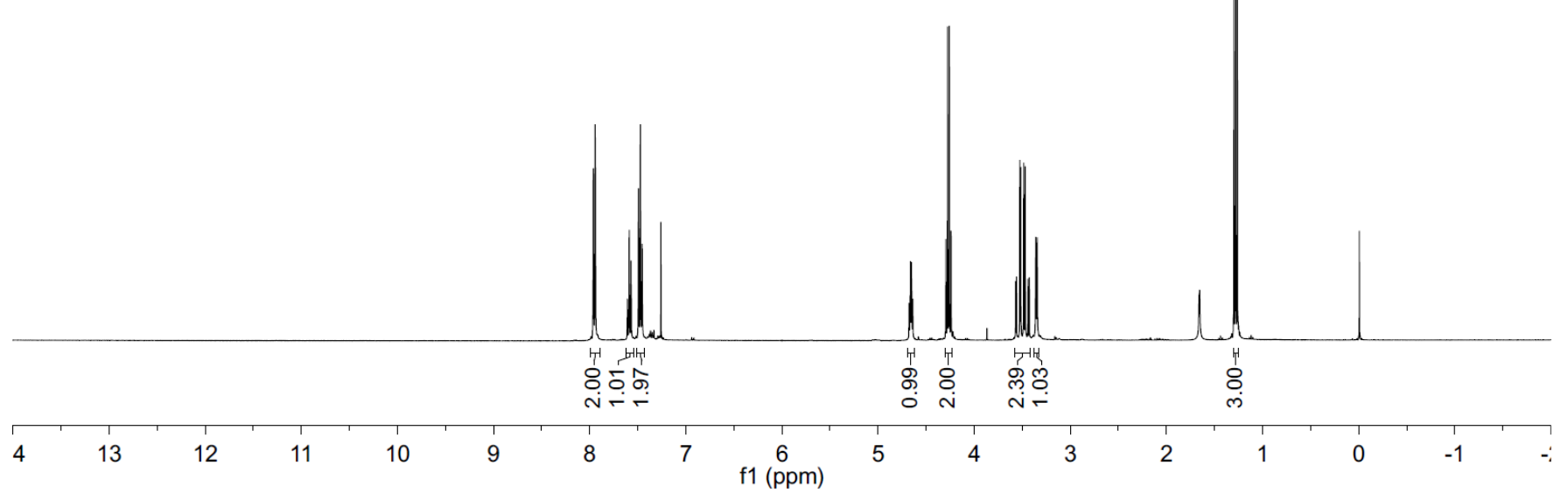

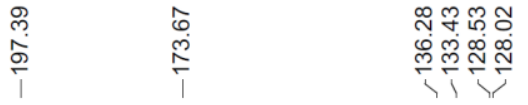

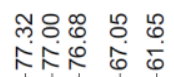

ஷิ

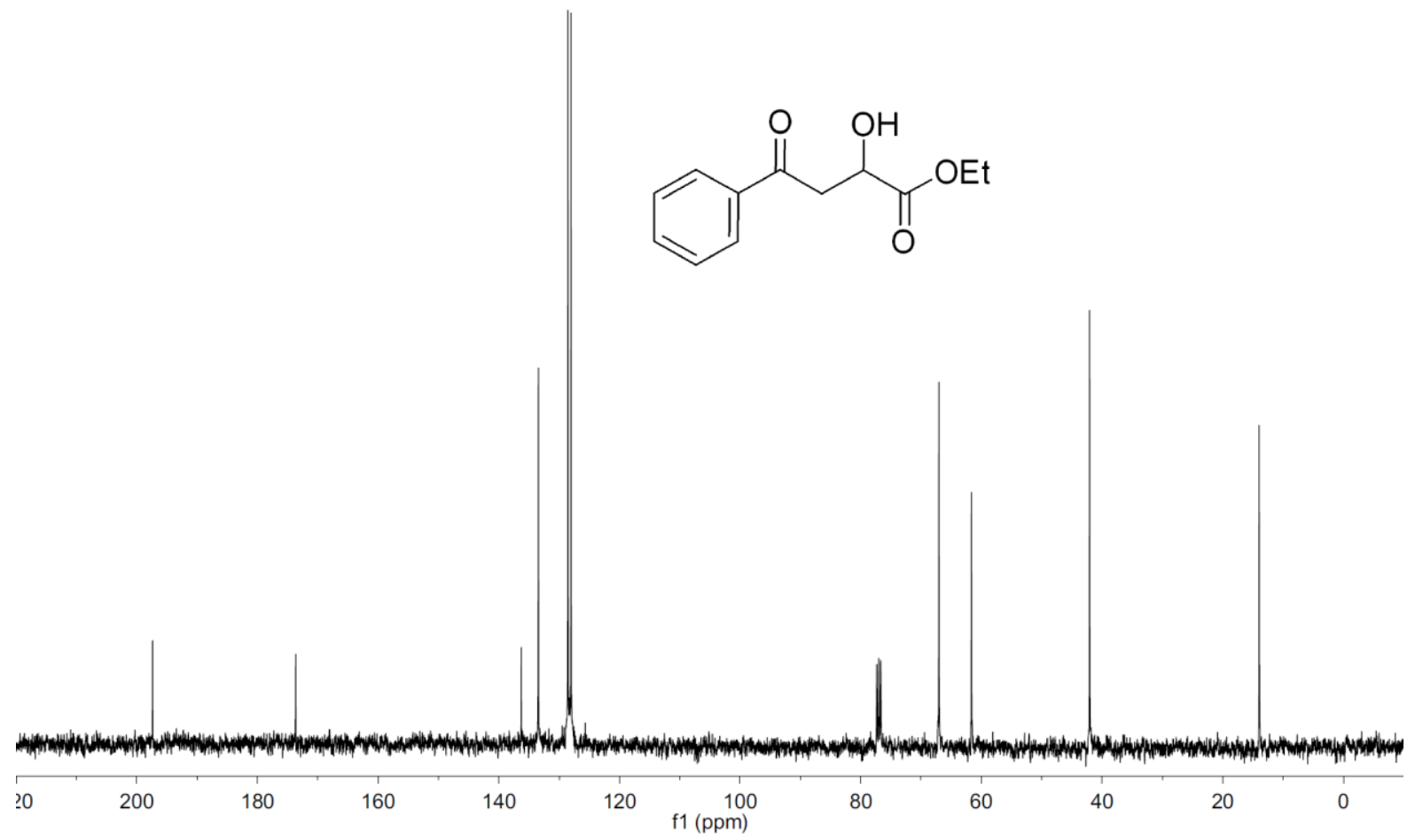


$4 i$

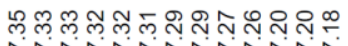

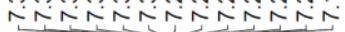

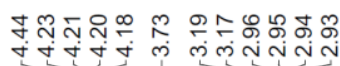

$\stackrel{5}{i}$

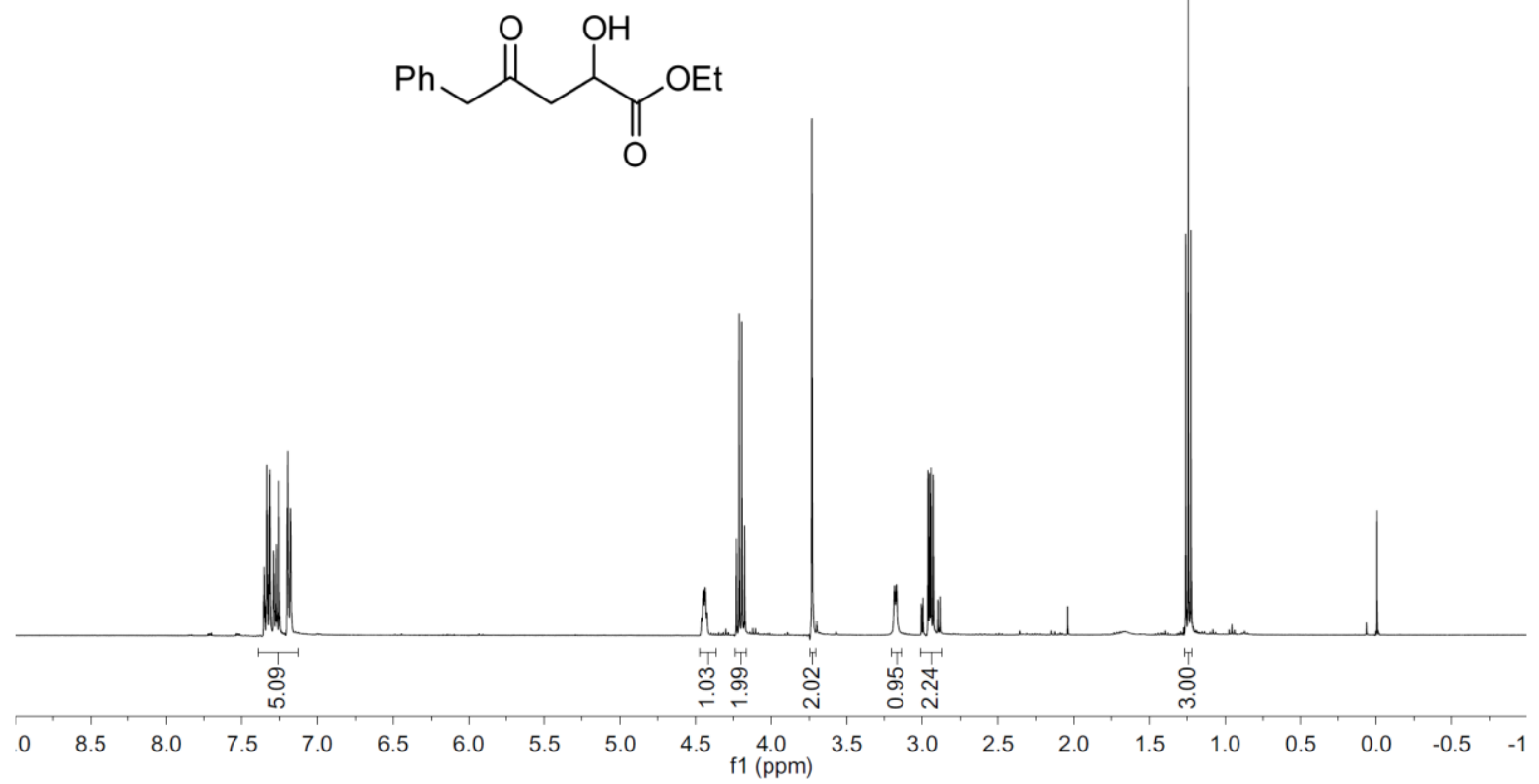

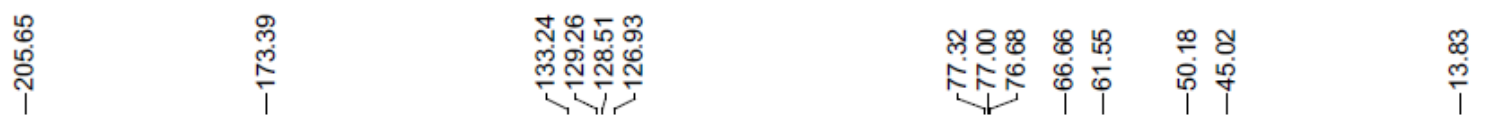

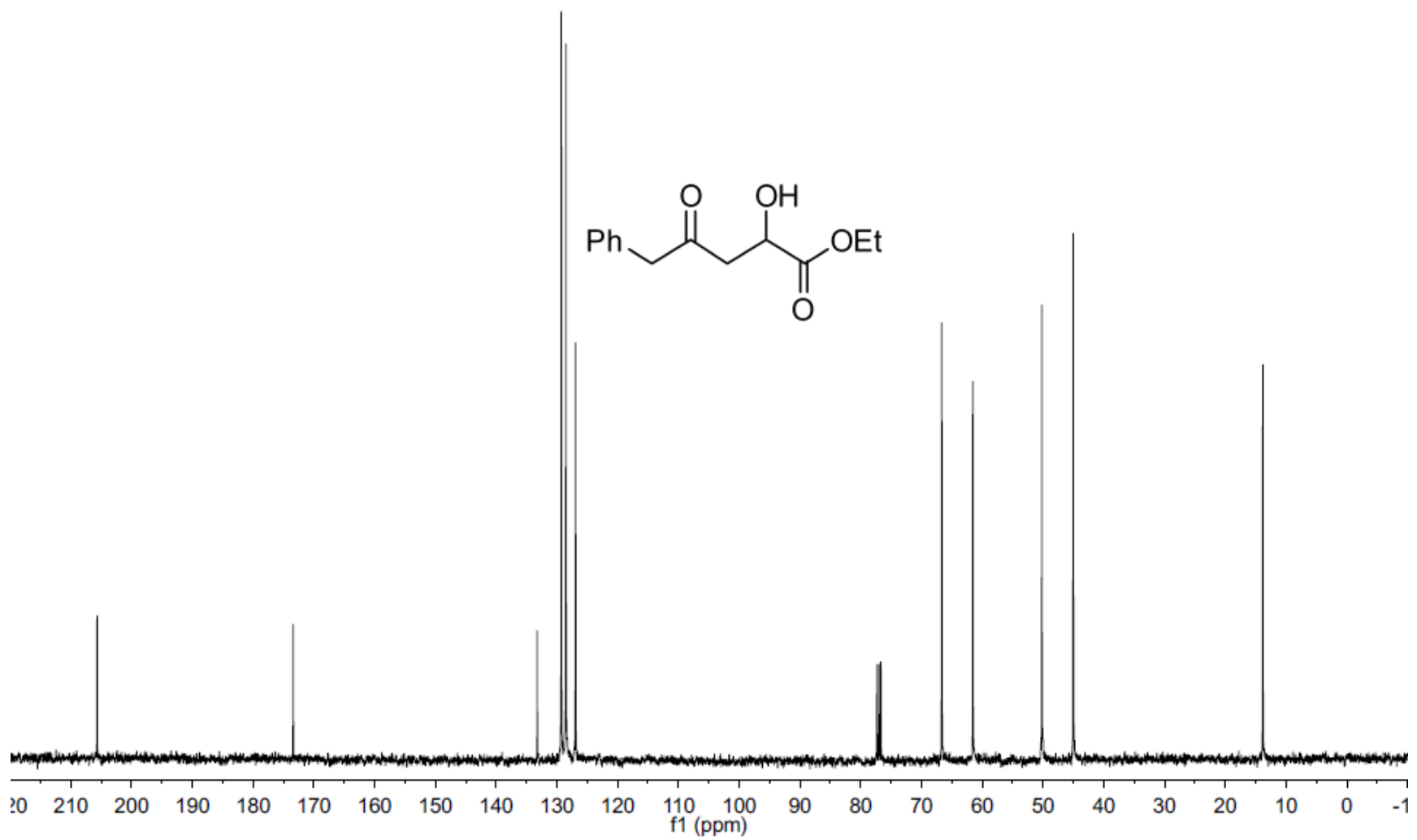




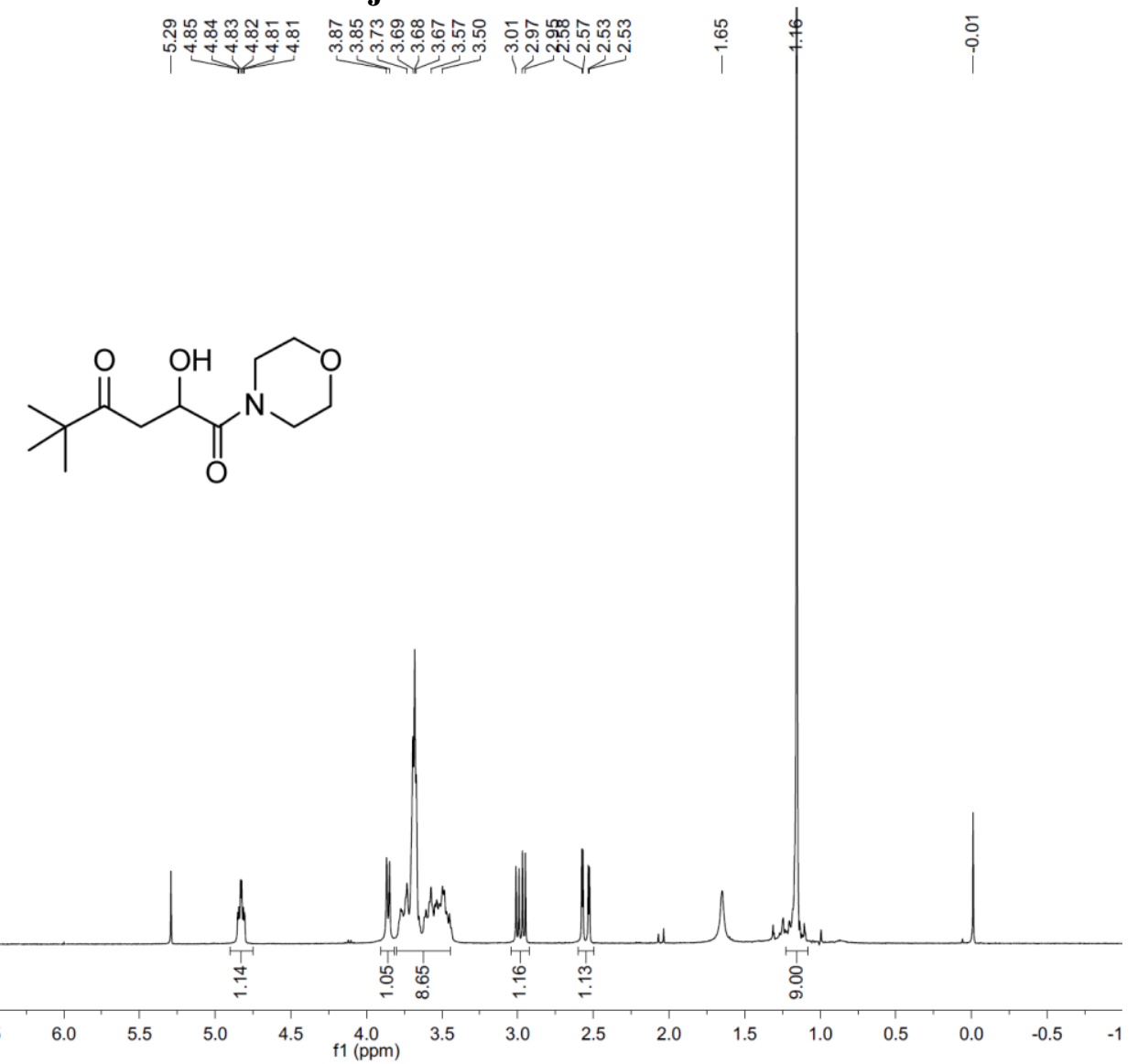

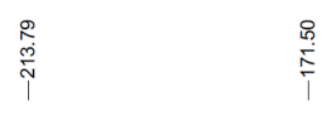

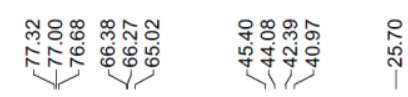<smiles>CC(C)(C)C(=O)CC(O)C(=O)N1CCOCC1</smiles>

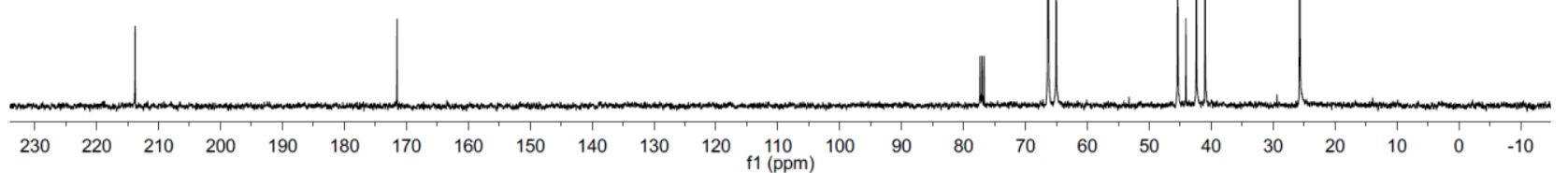




\section{HPLC diagram of chiral products}

(2a) Table 1, racemate of $2 a$

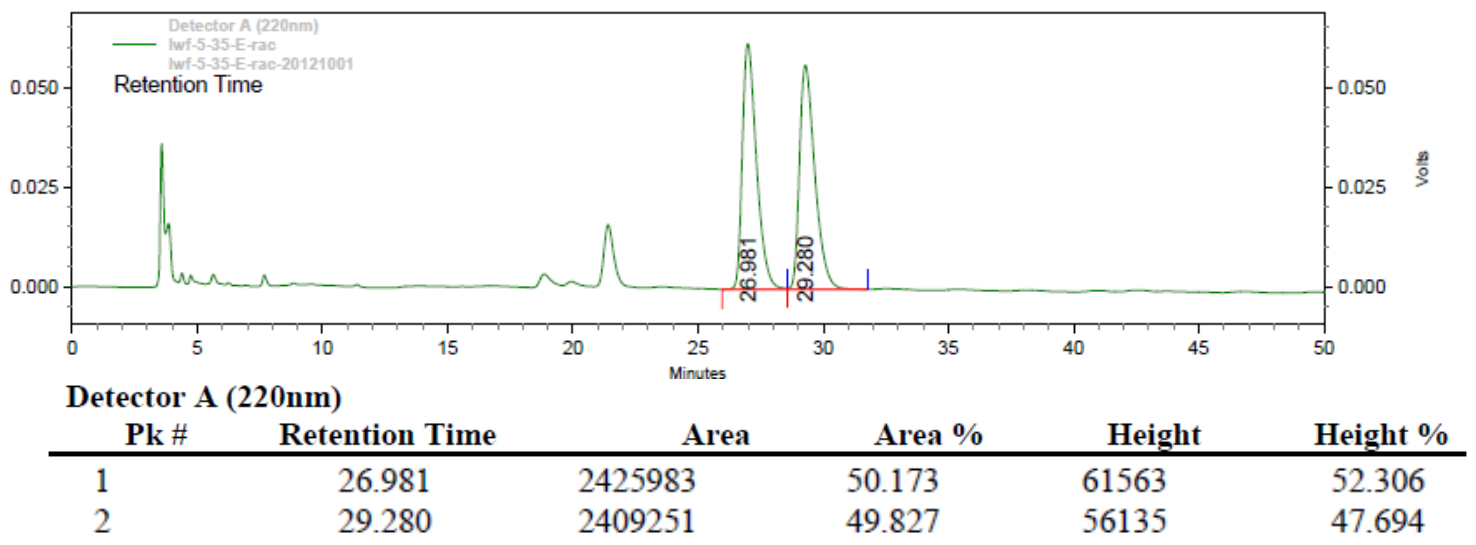

Table 1, entry 1: $\mathrm{Ru}-(S)-\mathrm{SunPhos}, 50{ }^{\circ} \mathrm{C}, 10$ bar $\mathrm{H}_{2}$

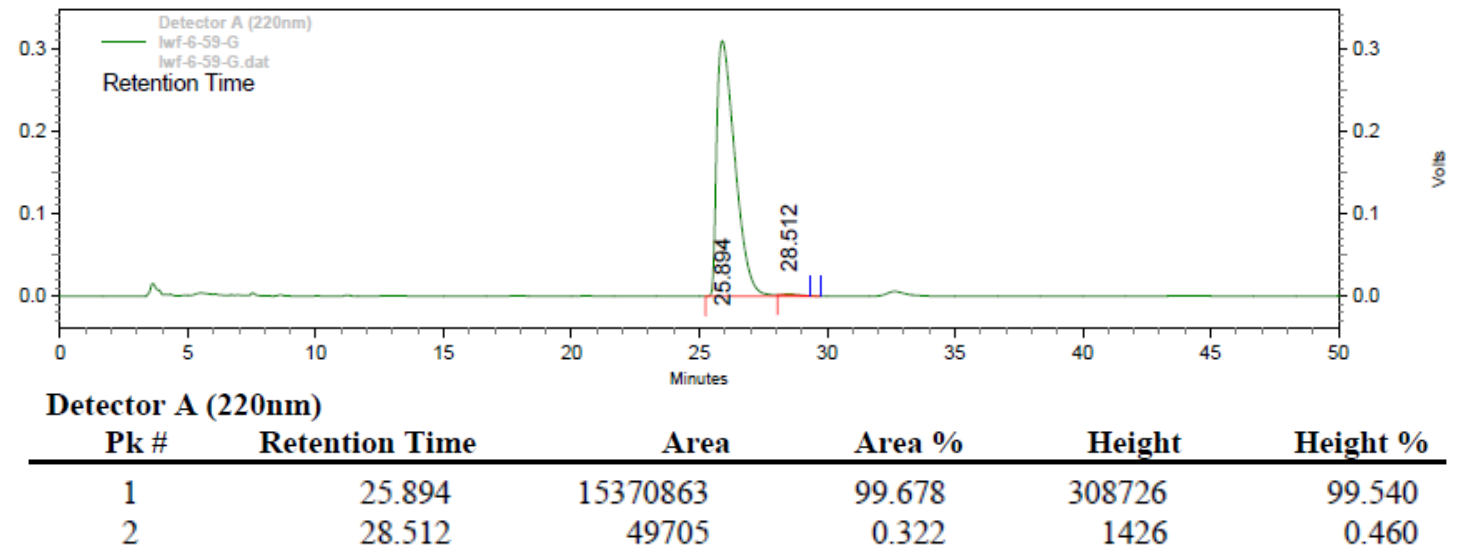

Table 1, entry 2 : Ru- $(S)$-SunPhos, $50{ }^{\circ} \mathrm{C}, 20$ bar $\mathrm{H}_{2}$

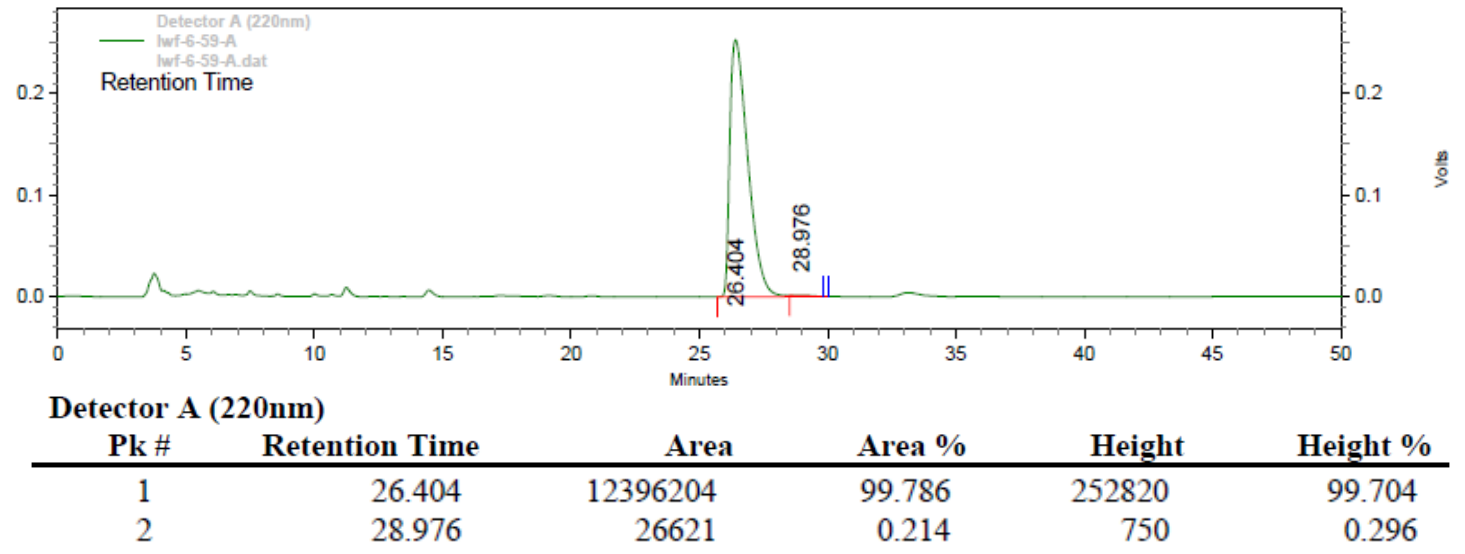

Table 1, entry 3 : $\mathrm{Ru}-(S)$-SunPhos, $70{ }^{\circ} \mathrm{C}, 20$ bar $\mathrm{H}_{2}$ 


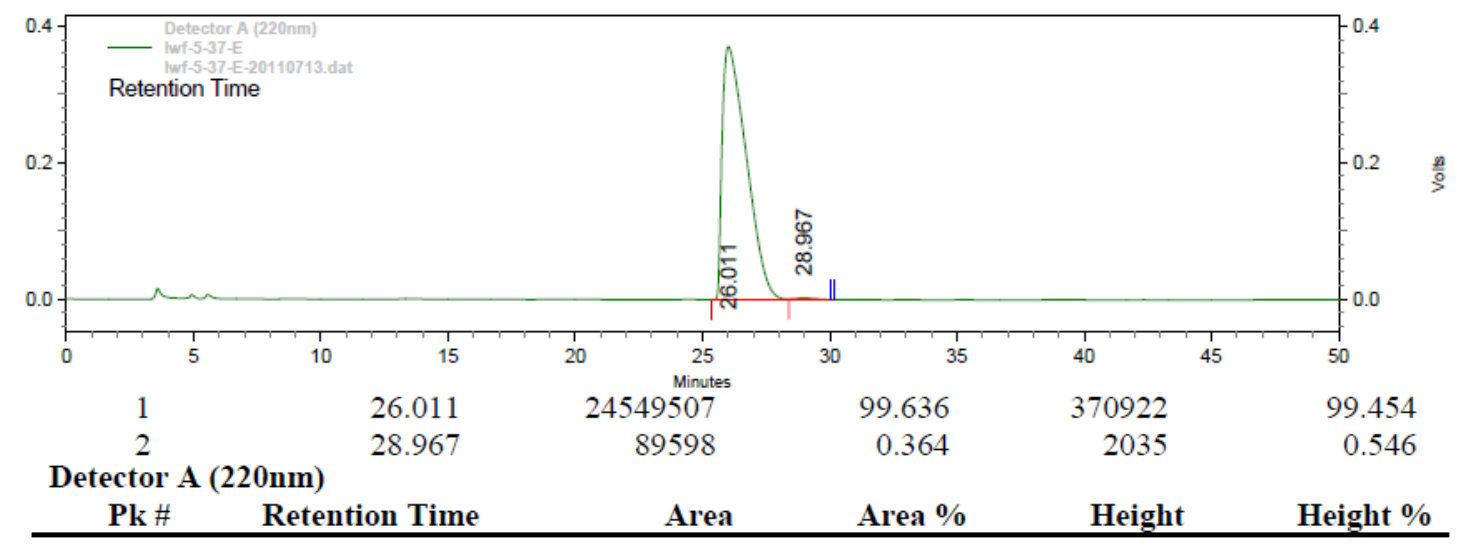

Table 1, entry 4: $\mathrm{Ru}-(S)-\mathrm{SunPhos}, 90{ }^{\circ} \mathrm{C}, 20$ bar $\mathrm{H}_{2}$

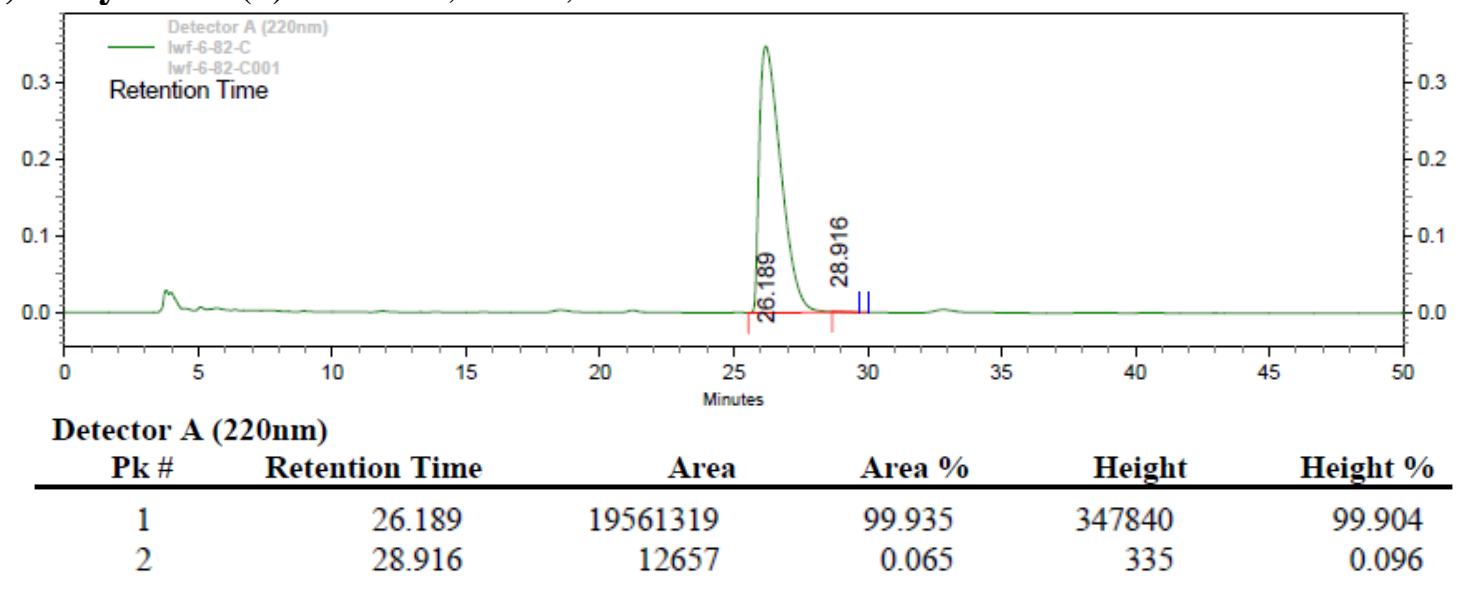

Table 1, entry 5: $\mathrm{Ru}-(S)-\mathrm{SegPhos}, 70{ }^{\circ} \mathrm{C}, 20$ bar $\mathrm{H}_{2}$

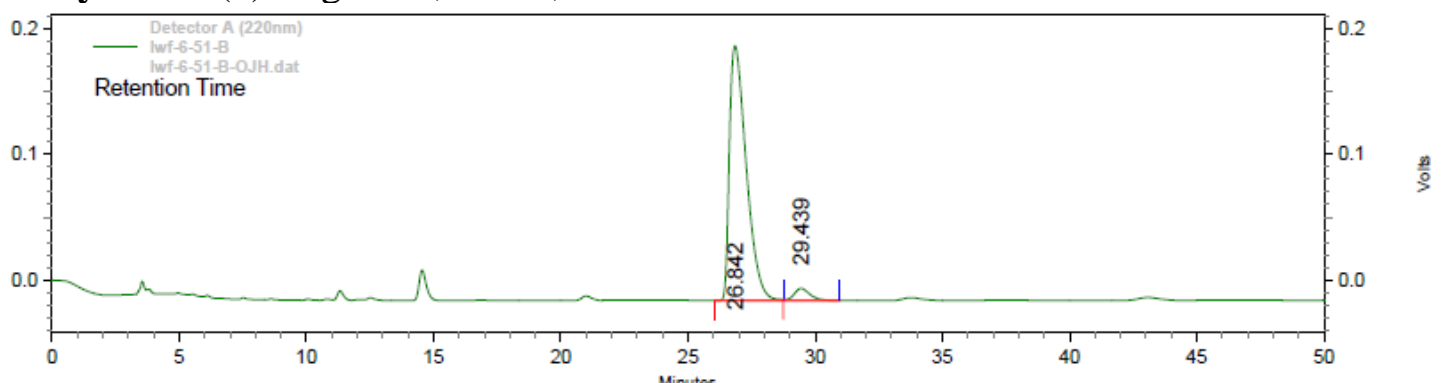

Detector A (220nm)

\begin{tabular}{ccccrr} 
Pk \# & Retention Time & Area & Area \% & Height & Height \% \\
\hline 1 & 26.842 & 9303591 & 95.730 & 202243 & 95.453 \\
2 & 29.439 & 415023 & 4.270 & 9634 & 4.547
\end{tabular}

Table 1, entry 6: $\mathrm{Ru}-(R)$-Binap , $70{ }^{\circ} \mathrm{C}, 20$ bar $\mathrm{H}_{2}$ 


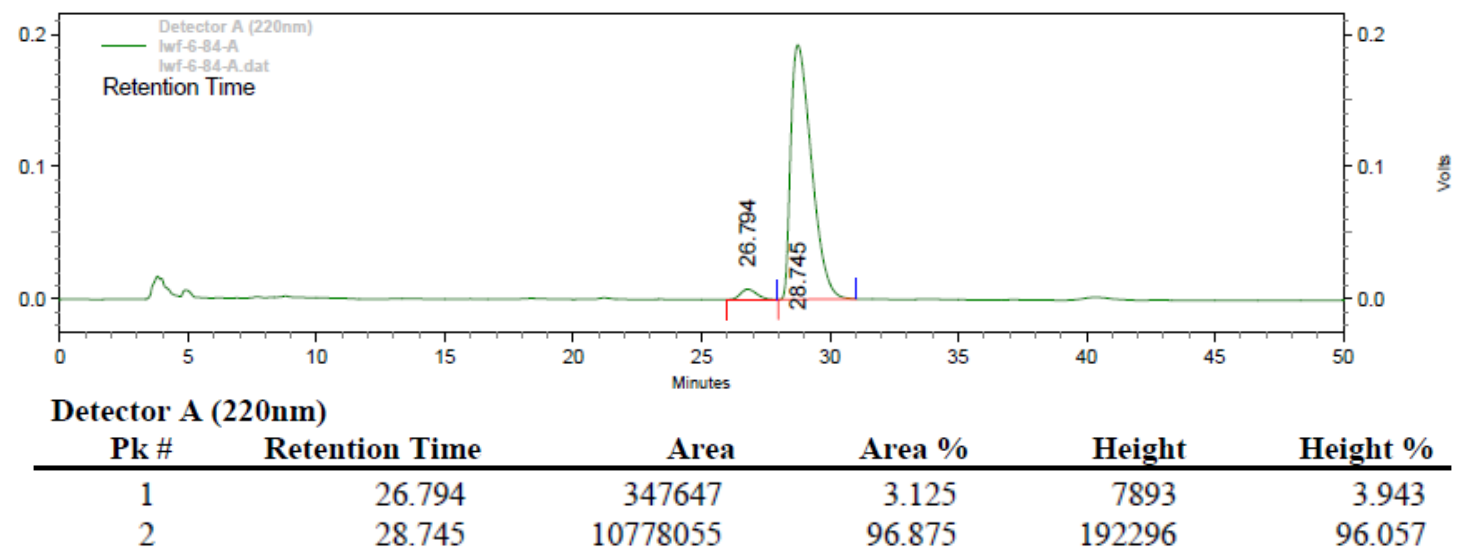

Table S1, entry 3: $\mathrm{Ru}-(S)$-SunPhos, 1,4-dioxane, $70{ }^{\circ} \mathrm{C}, 20$ bar $\mathrm{H}_{2}$

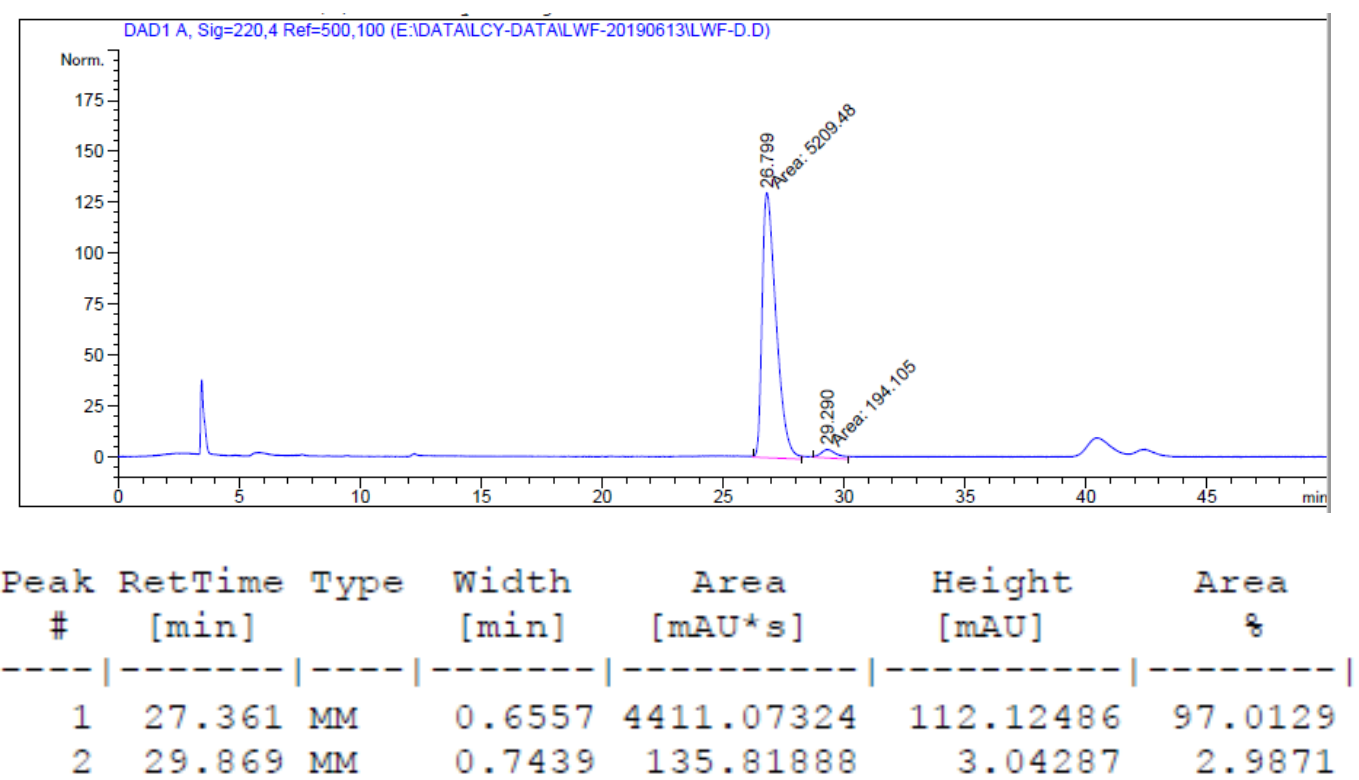

Table S1, entry 4: $\mathrm{Ru}-(S)-\mathrm{SunPhos}$, EtOAc, $70{ }^{\circ} \mathrm{C}, 20 \mathrm{bar} \mathrm{H}_{2}$

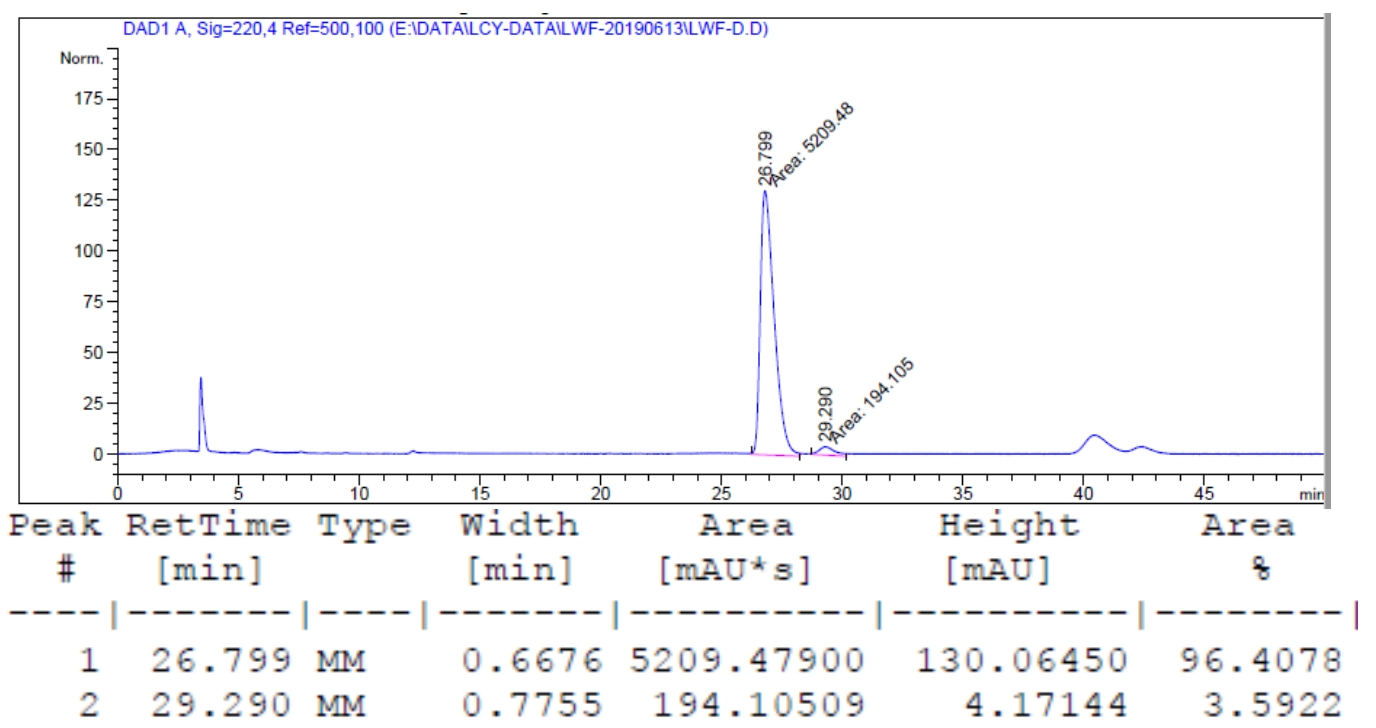


(2b) Table 2, entry 1

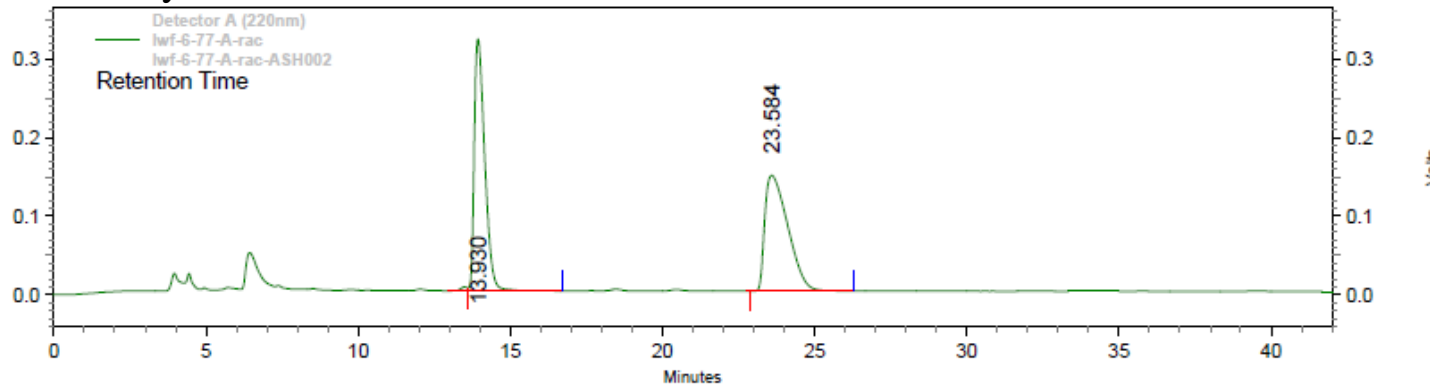

Detector A (220nm)

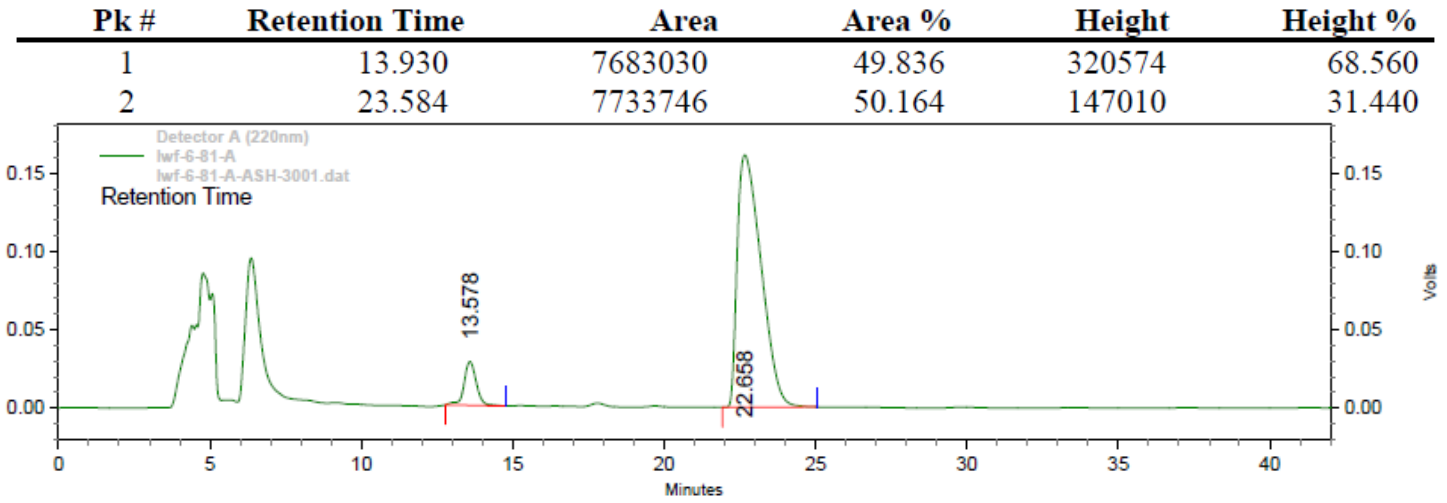

Detector A (220nm)

\begin{tabular}{cccccc} 
Pk \# & Retention Time & Area & Area \% & Height & Height \% \\
\hline 1 & 13.578 & 784542 & 8.134 & 27910 & 14.742 \\
2 & 22.658 & 8860720 & 91.866 & 161414 & 85.258
\end{tabular}

(2c) Table 2, entry 2

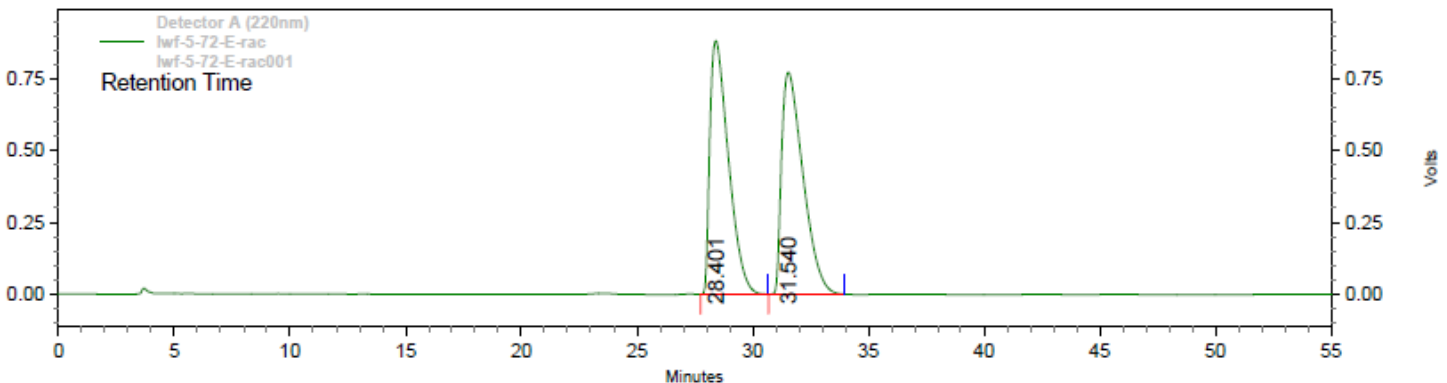

Detector A (220nm)

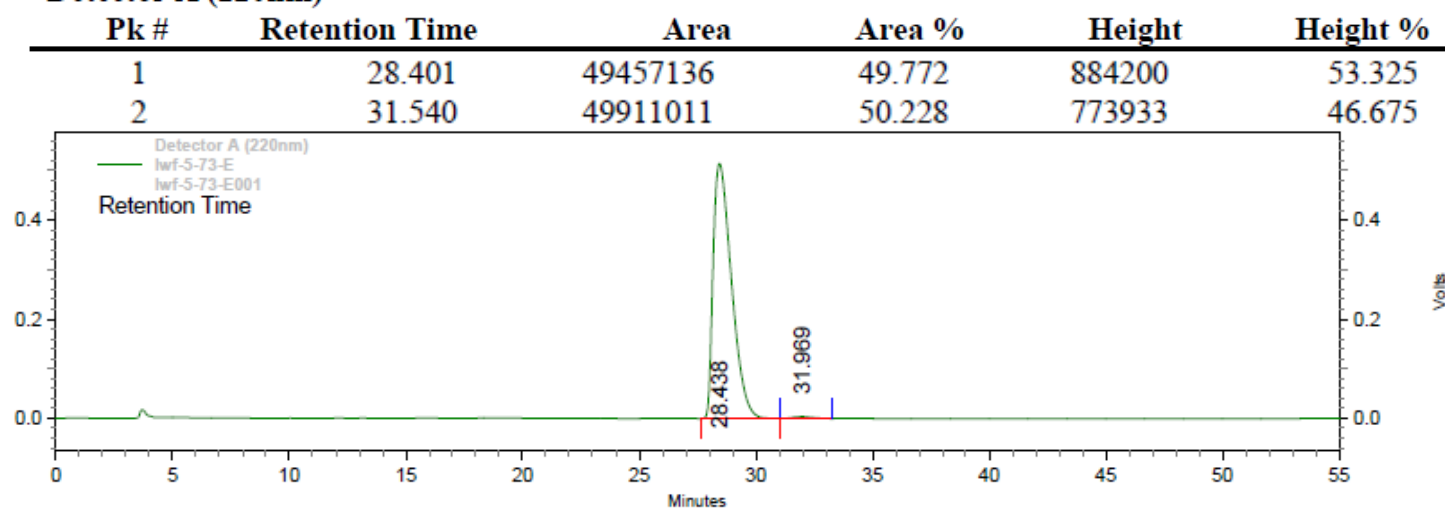

Detector A (220nm)

Pk\# 


$\begin{array}{rrrrrr}1 & 28.438 & 28198335 & 99.295 & 514823 & 99.324 \\ 2 & 31.969 & 200074 & 0.705 & 3504 & 0.676\end{array}$

(2d) Table 2, entry 3

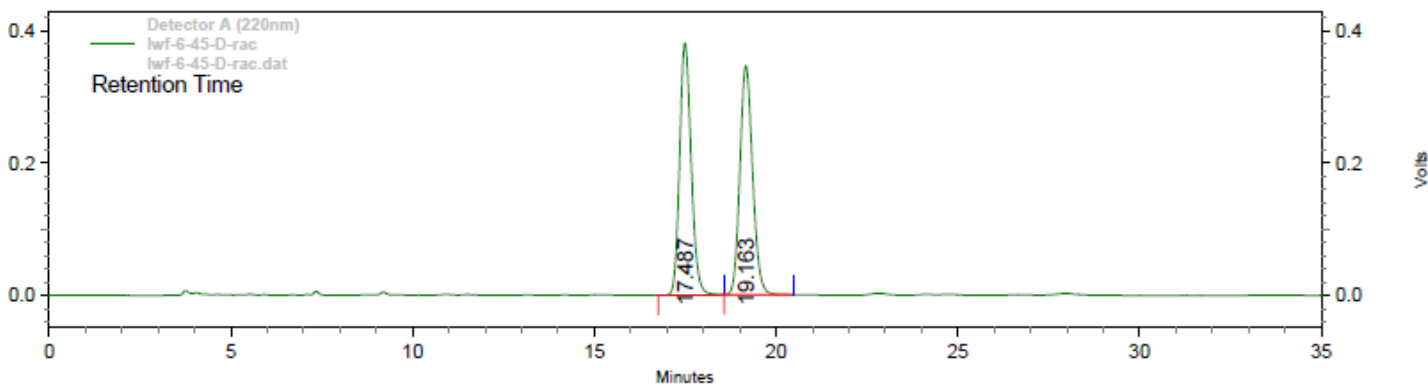

Detector A (220nm)

\begin{tabular}{crrrrr} 
Pk \# & Retention Time & Area & Area \% & Height & Height \% \\
\hline 1 & 17.487 & 8446290 & 49.937 & 381894 & 52.395 \\
2 & 19.163 & 8467723 & 50.063 & 346982 & 47.605
\end{tabular}

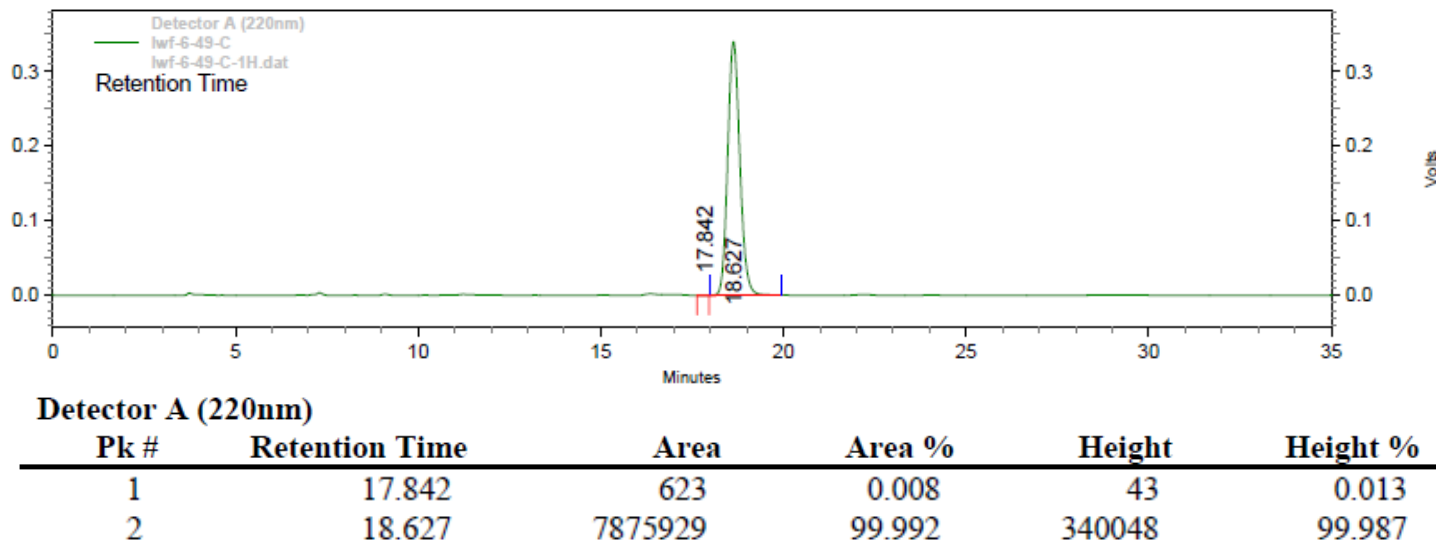

(2e) Table 2 , entry 4

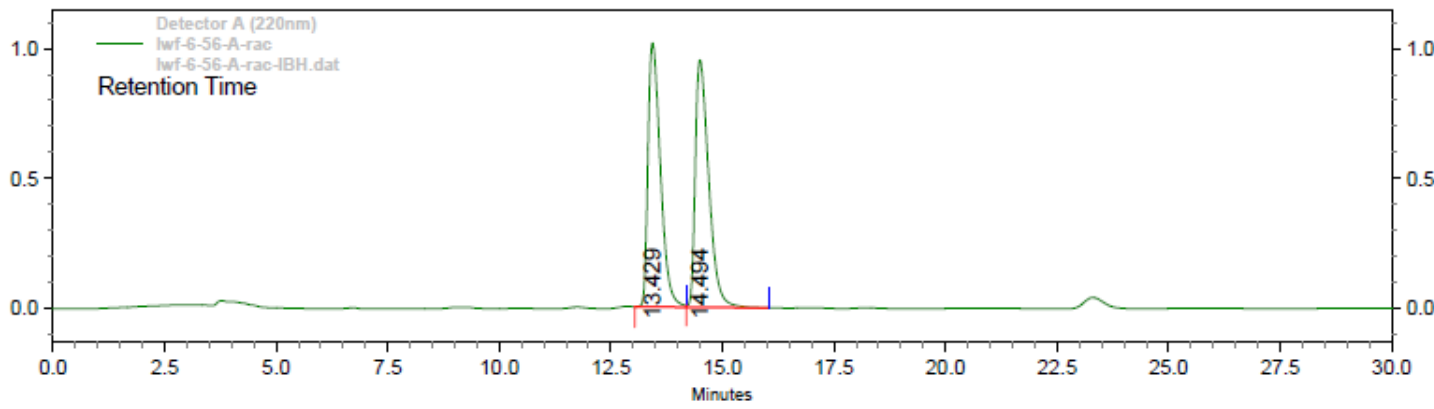

Detector A (220nm)

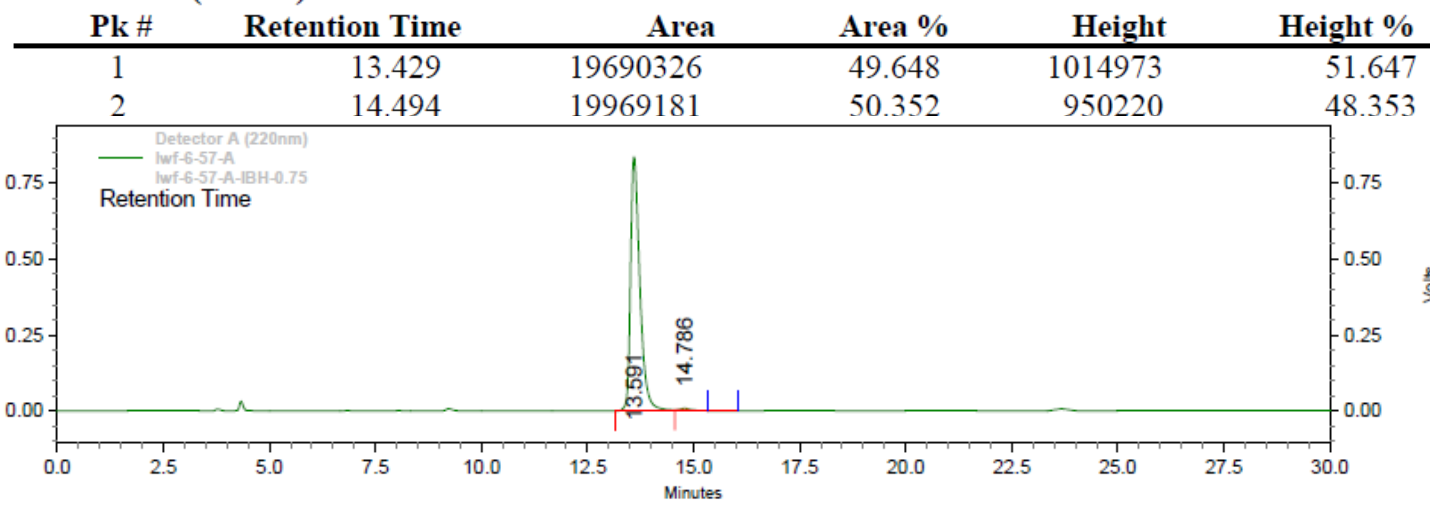

Detector A (220nm)

Pk \# Retention Time

Area

Area \%

Height

Height \% 


$\begin{array}{rrrrrr}1 & 13.591 & 12956197 & 99.353 & 836015 & 99.299 \\ 2 & 14.786 & 84348 & 0.647 & 5901 & 0.701\end{array}$

(2f) Table 2, entry 5

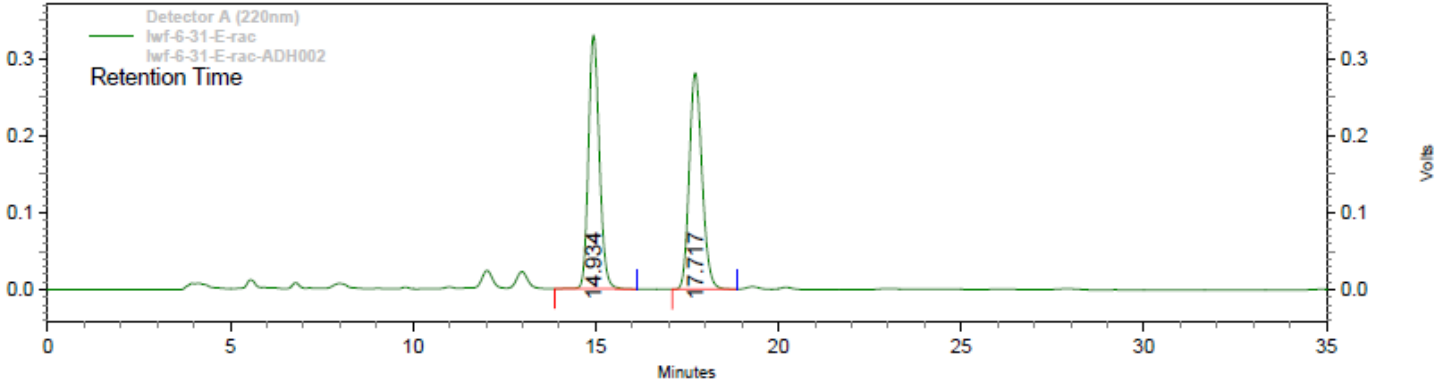

Detector A (220nm)

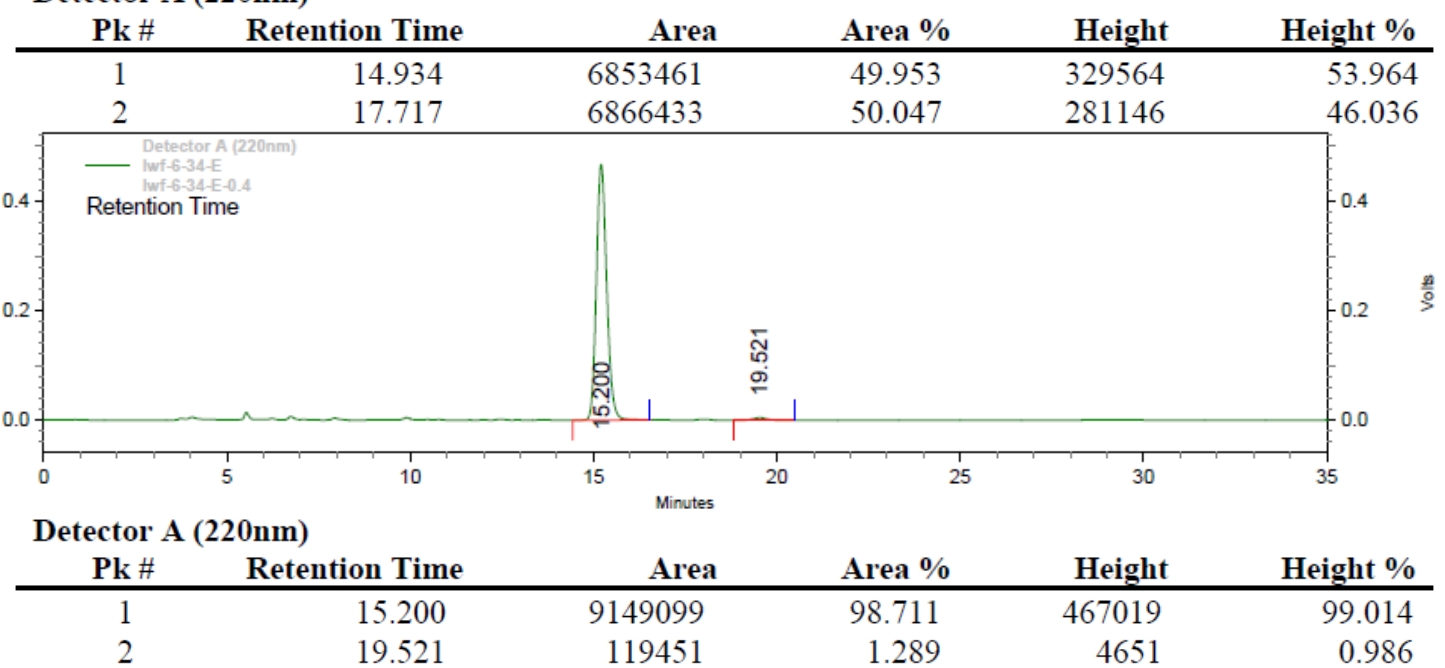

(2h) Table 2, entry 7

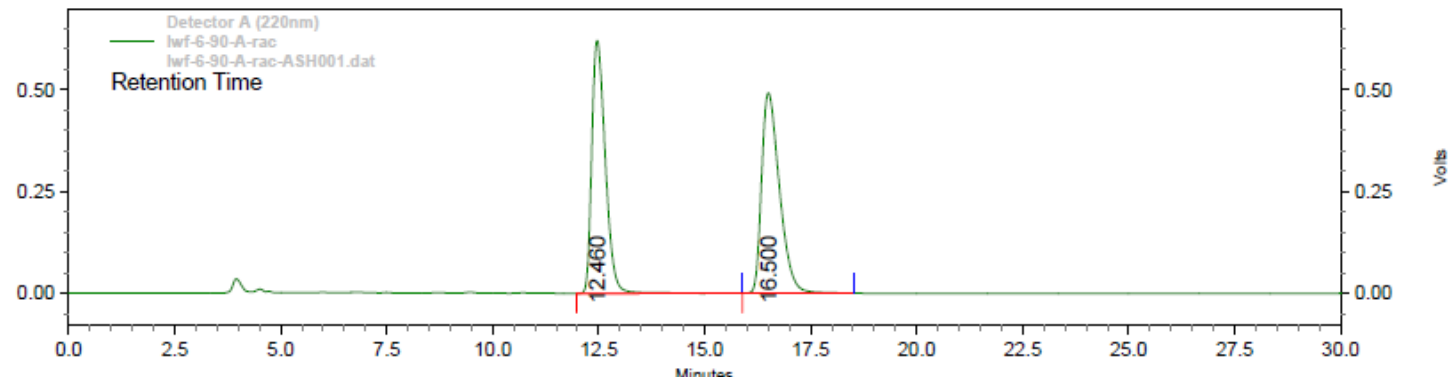

Detector A (220nm)

Pk \# Retention Time

Area

Area \%

Height

Height \%

$\begin{array}{llllll}1 & 12.460 & 13807978 & 48.863 & 622017 & 55.808 \\ 2 & 16.500 & 14450430 & 51.137 & 492544 & 44.192\end{array}$




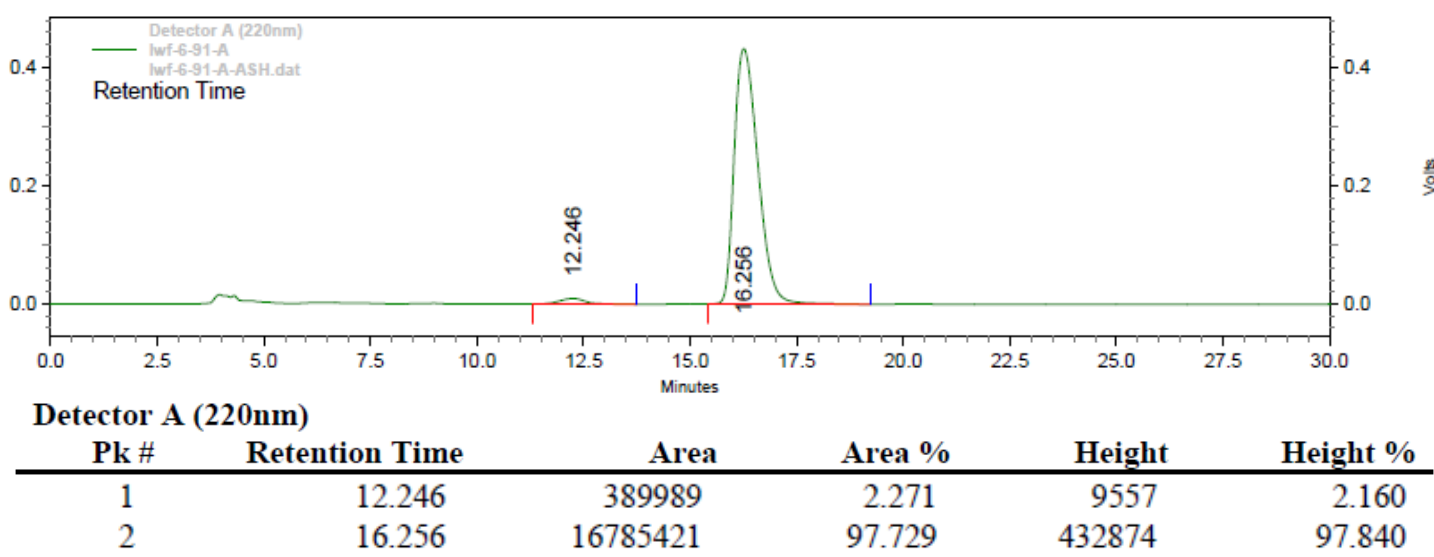

(2i) Table 2, entry 8

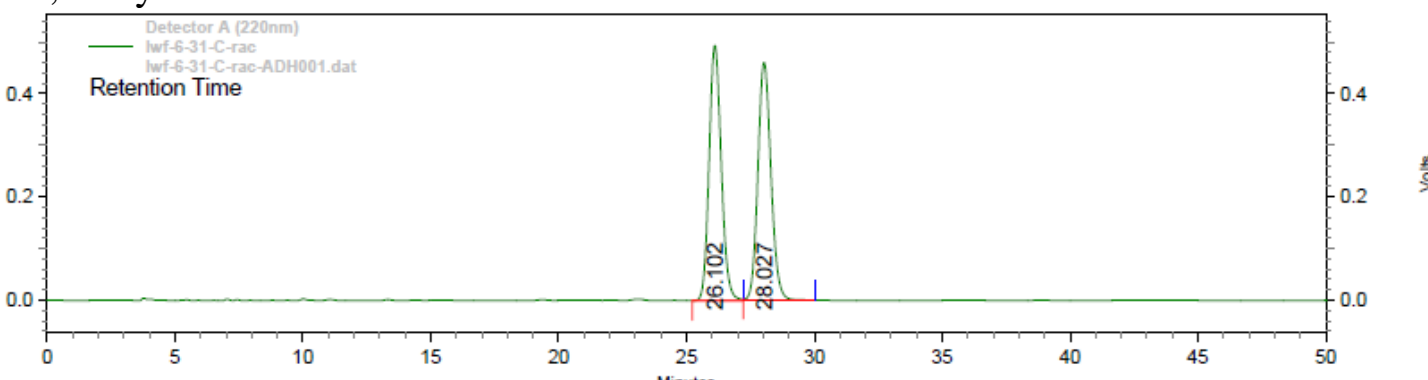

Detector A (220nm)

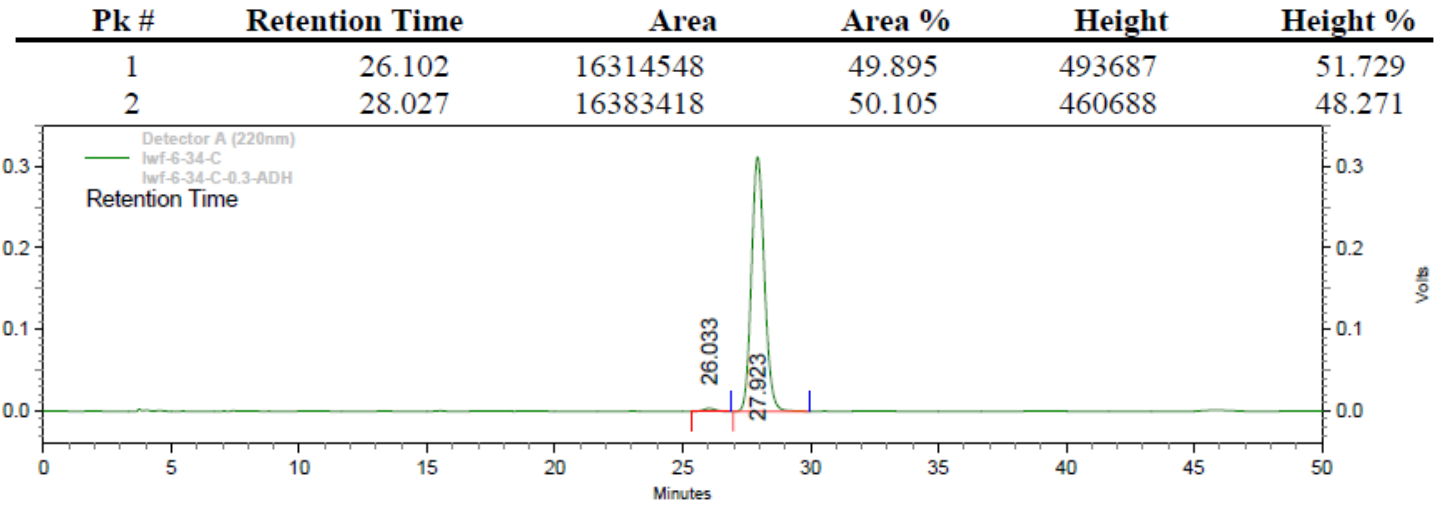

Detector A (220nm)

\begin{tabular}{ccrrrr} 
Pk \# & Retention Time & Area & Area \% & Height & Height \% \\
\hline 1 & 26.033 & 121238 & 1.089 & 3767 & 1.194 \\
2 & 27.923 & 11010327 & 98.911 & 311757 & 98.806
\end{tabular}

(2j) Table 2, entry 9

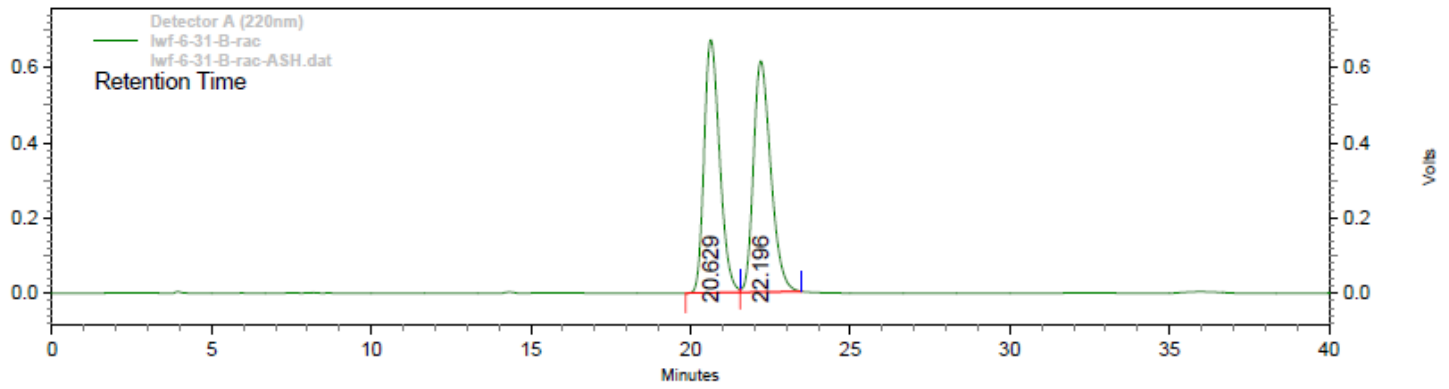


Detector A (220nm)

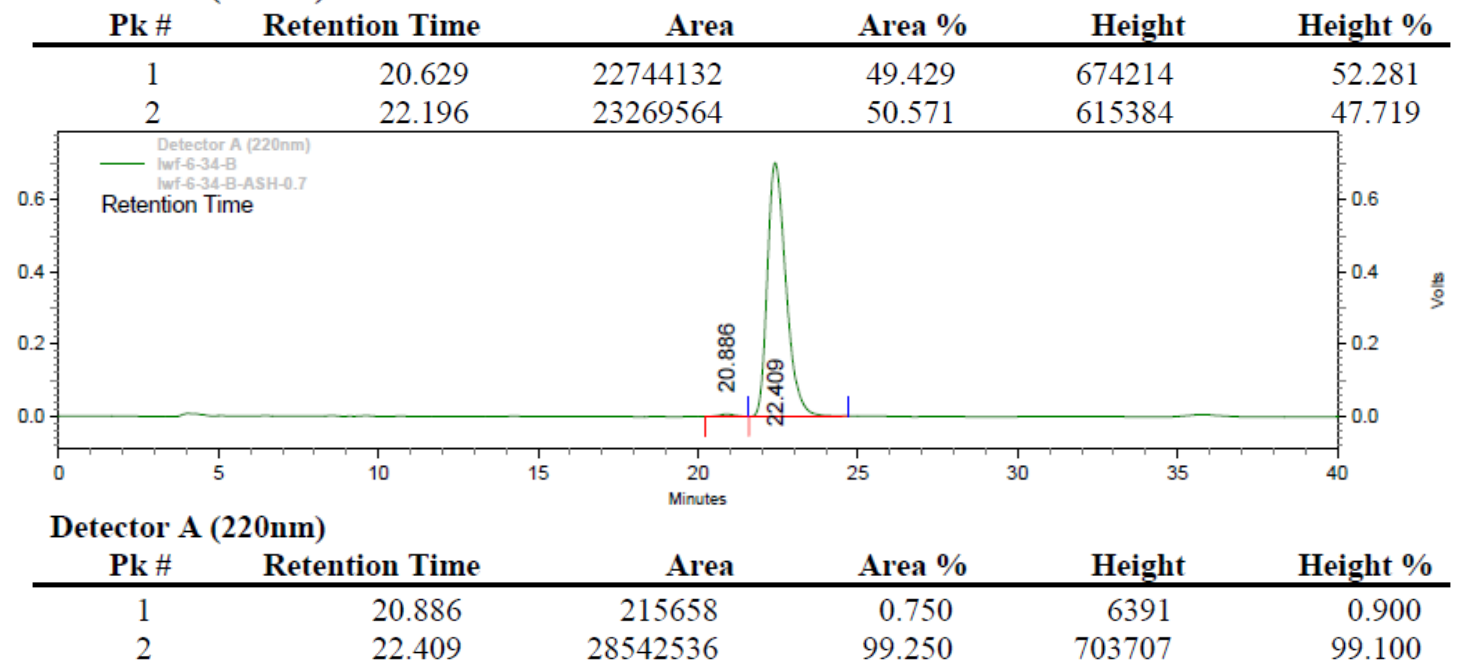

(2k) Table 2, entry 10

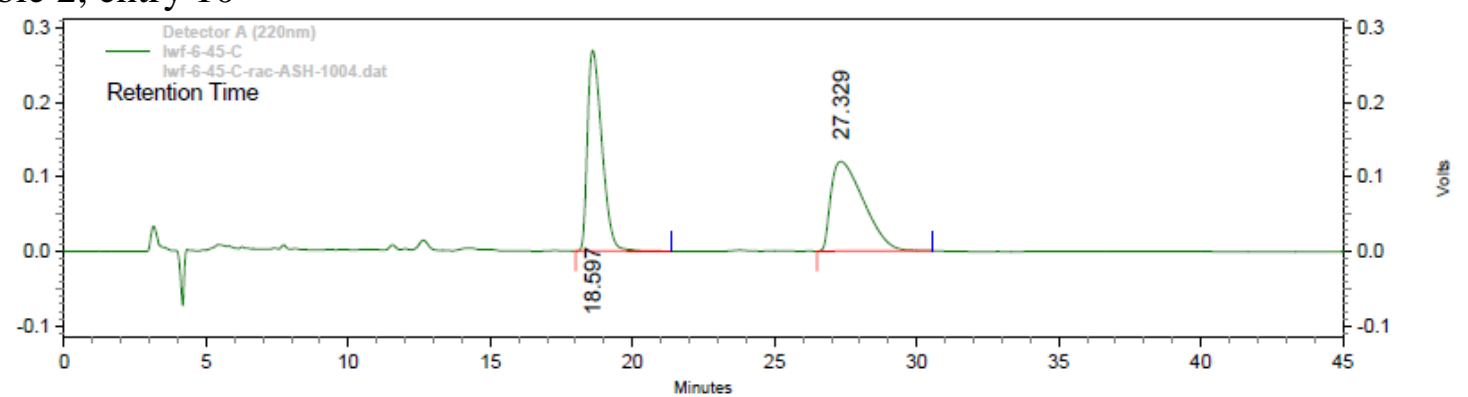

Detector A (220nm)

\begin{tabular}{cccccc} 
Pk \# & Retention Time & Area & Area \% & Height & Height \% \\
\hline 1 & 18.597 & 9540886 & 49.836 & 269238 & 69.178 \\
2 & 27.329 & 9603844 & 50.164 & 119955 & 30.822
\end{tabular}

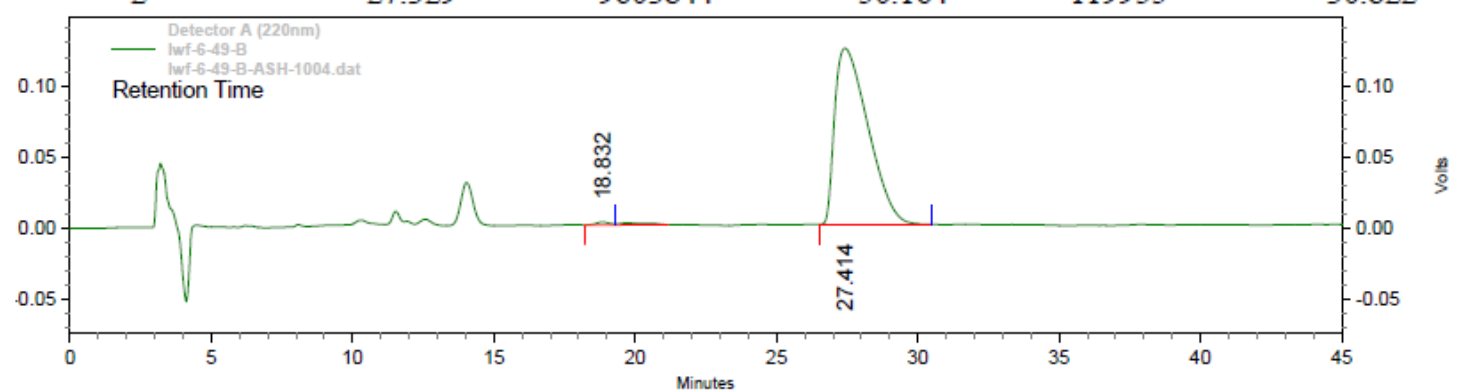

Detector A (220nm)

\begin{tabular}{cccccc} 
Pk\# & Retention Time & Area & Area \% & Height & Height \% \\
\hline 1 & 18.832 & 69387 & 0.681 & 2057 & 1.640 \\
2 & 27.414 & 10118010 & 99.319 & 123333 & 98.360
\end{tabular}

(4a) Table 3, entry 1

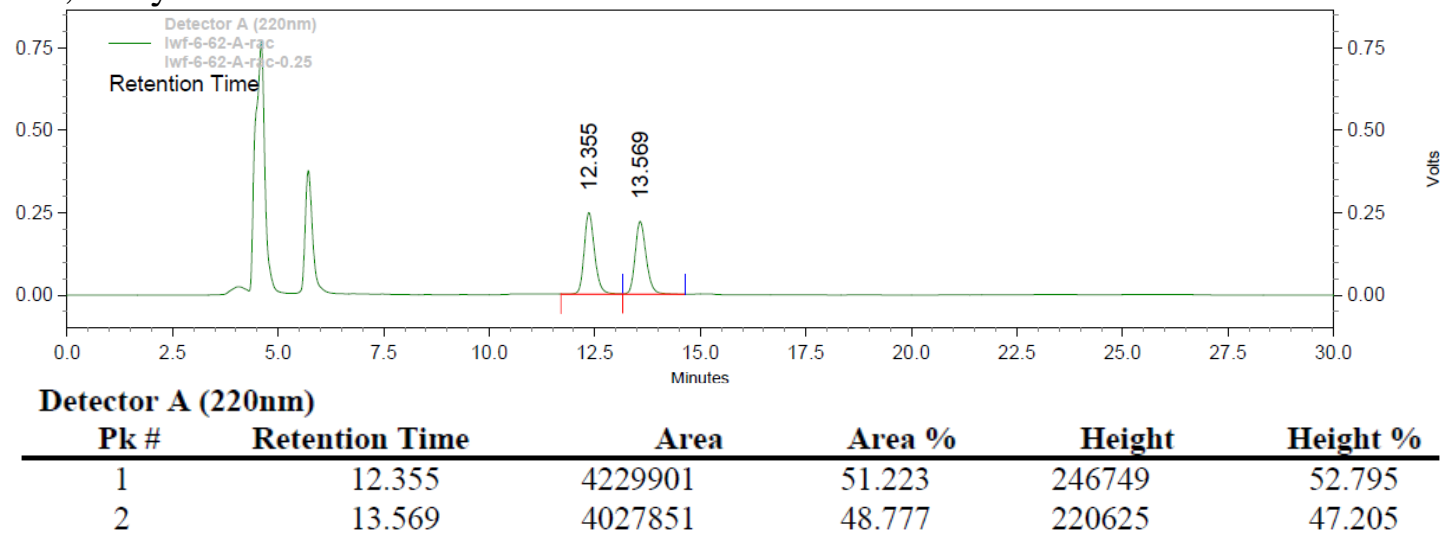


$50{ }^{\circ} \mathrm{C}$

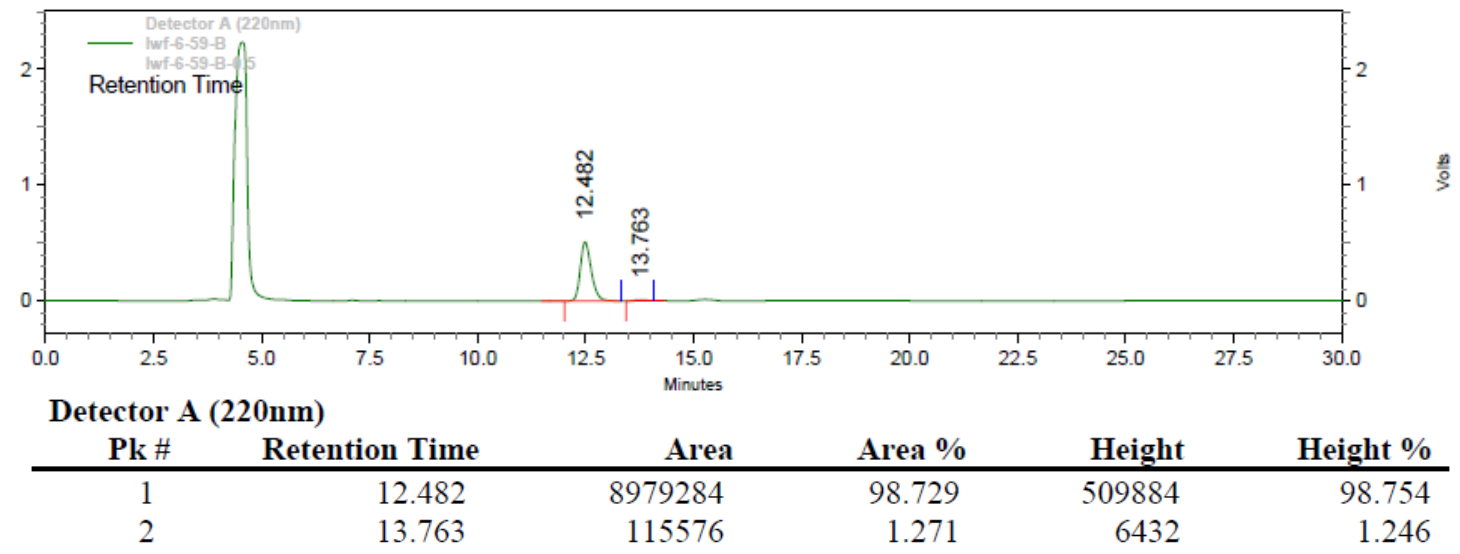

$70{ }^{\circ} \mathrm{C}, \quad 0.05 \mathrm{mmol} \mathrm{CaCO}_{3}$ was added:

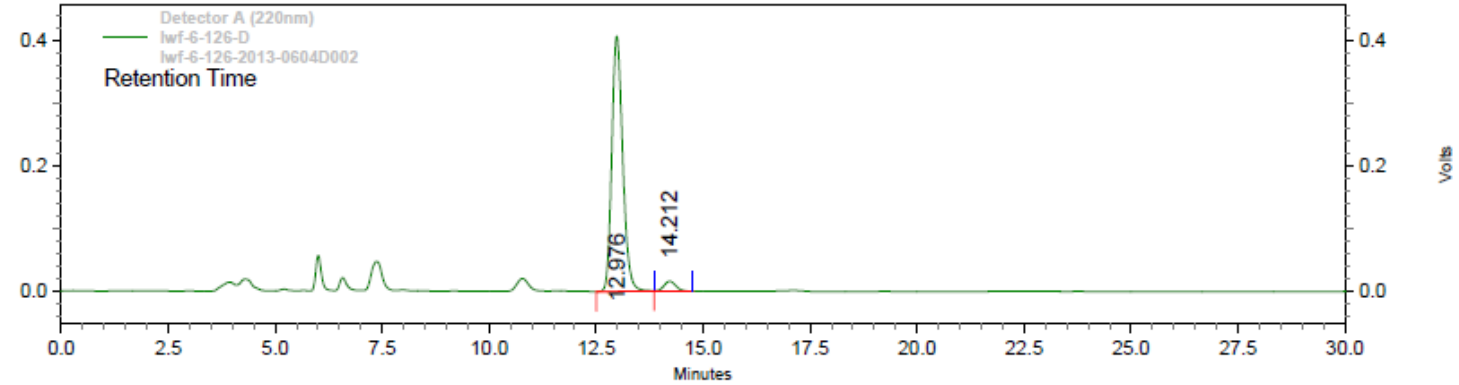

Detector A (220nm)

\begin{tabular}{cccccc} 
Pk \# & Retention Time & Area & Area \% & Height & Height \%o \\
\hline 1 & 12.976 & 7255379 & 95.807 & 407740 & 96.157 \\
2 & 14.212 & 317555 & 4.193 & 16297 & 3.843
\end{tabular}

(4b) Table 3, entry 2

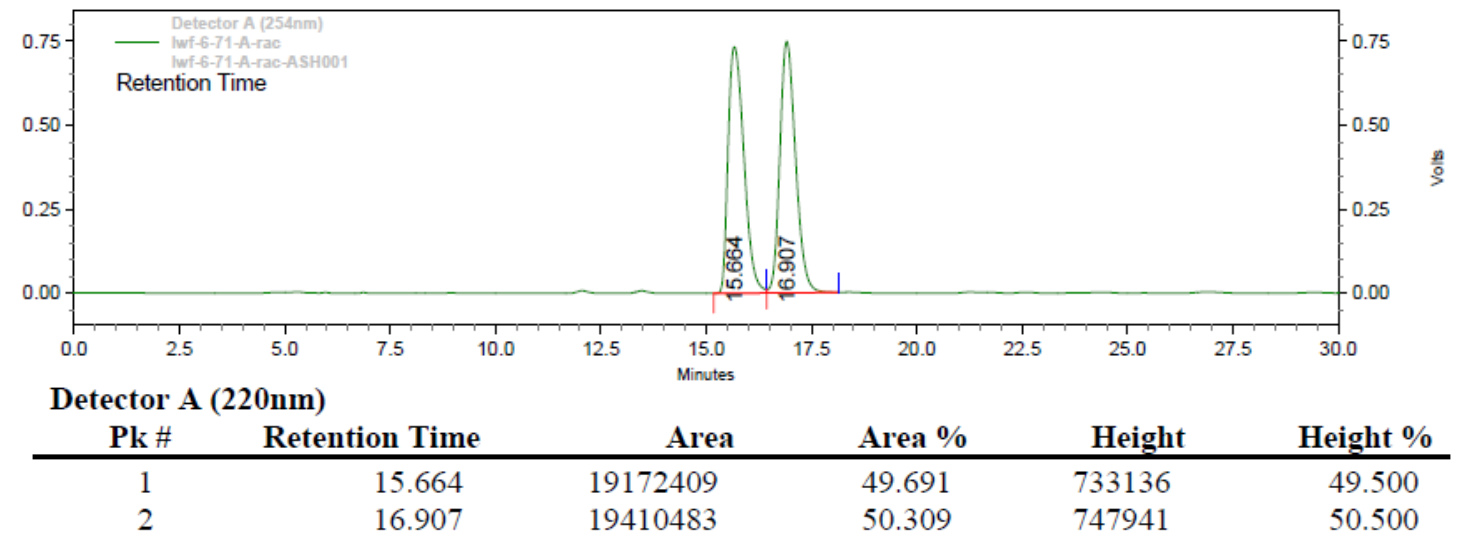




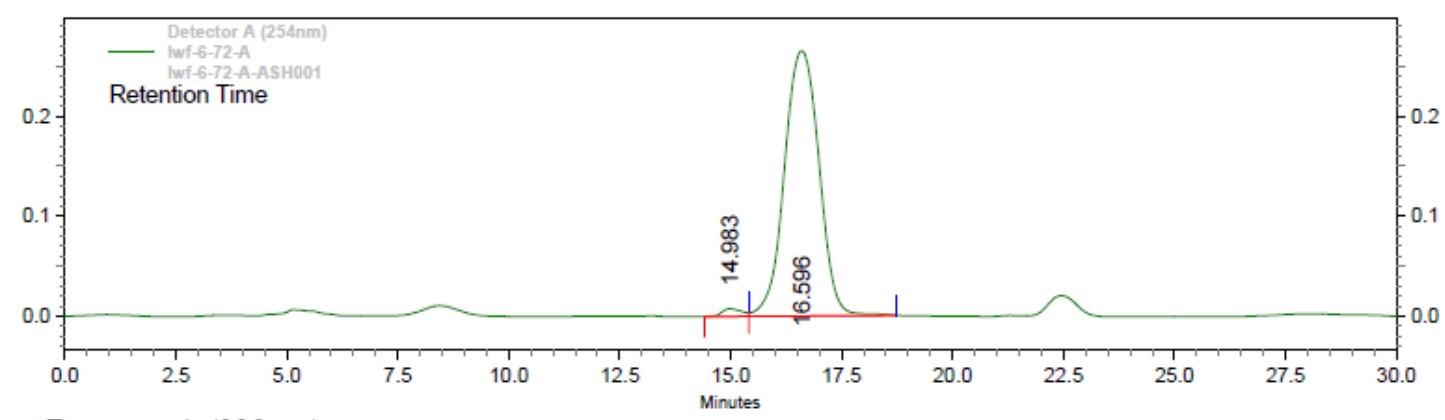

Detector A (220nm)

\begin{tabular}{cccrrr} 
Pk \# & Retention Time & Area & Area \% & Height & Height \% \\
\hline 1 & 14.983 & 238748 & 1.630 & 7589 & 2.783 \\
2 & 16.596 & 14405608 & 98.370 & 265071 & 97.217
\end{tabular}

(4c) Table 3, entry 3

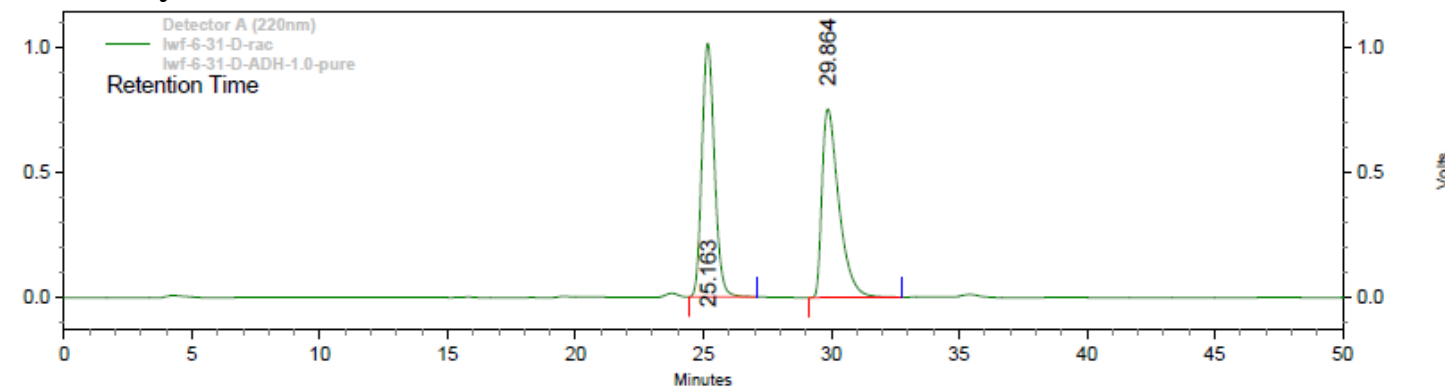

Detector A (220nm)

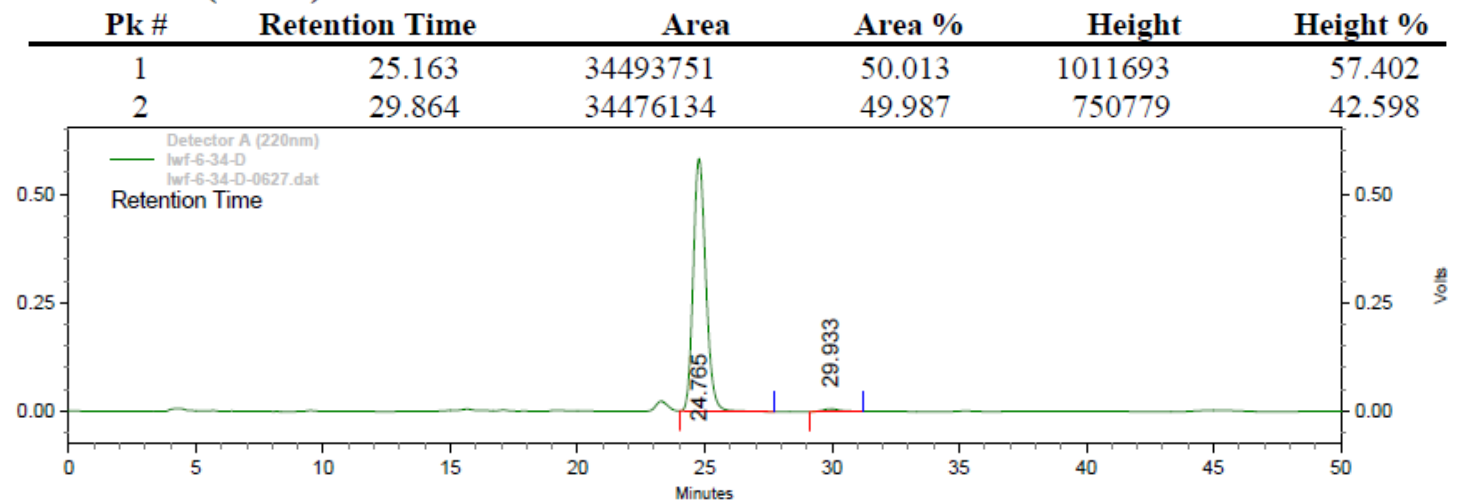

Detector A (220nm)

\begin{tabular}{ccccrr} 
Pk \# & Retention Time & Area & Area \% & Height & Height \% \\
\hline 1 & 24.765 & 20078245 & 98.789 & 582999 & 99.003 \\
2 & 29.933 & 246128 & 1.211 & 5874 & 0.997
\end{tabular}

(4d) Table 3, entry 4

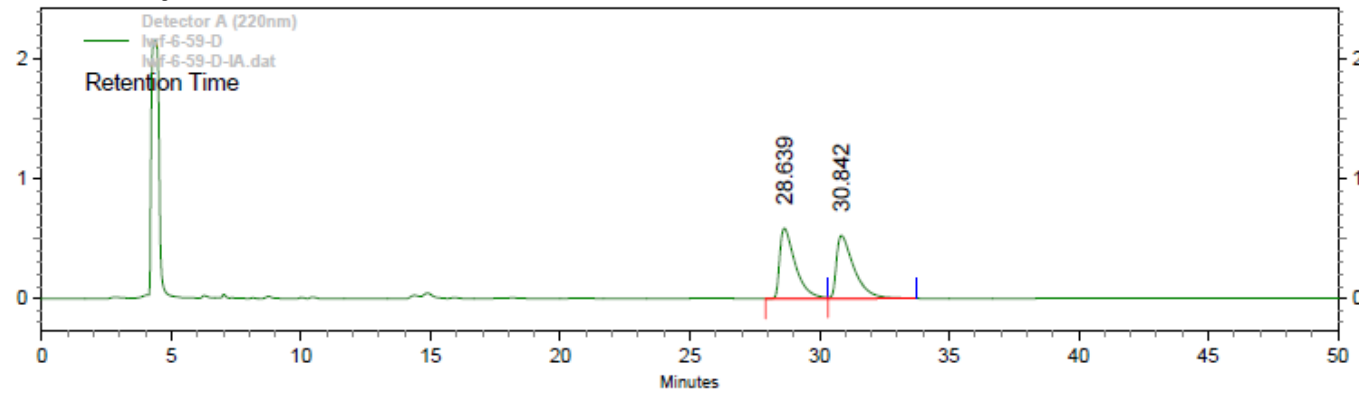


Detector A (220nm)

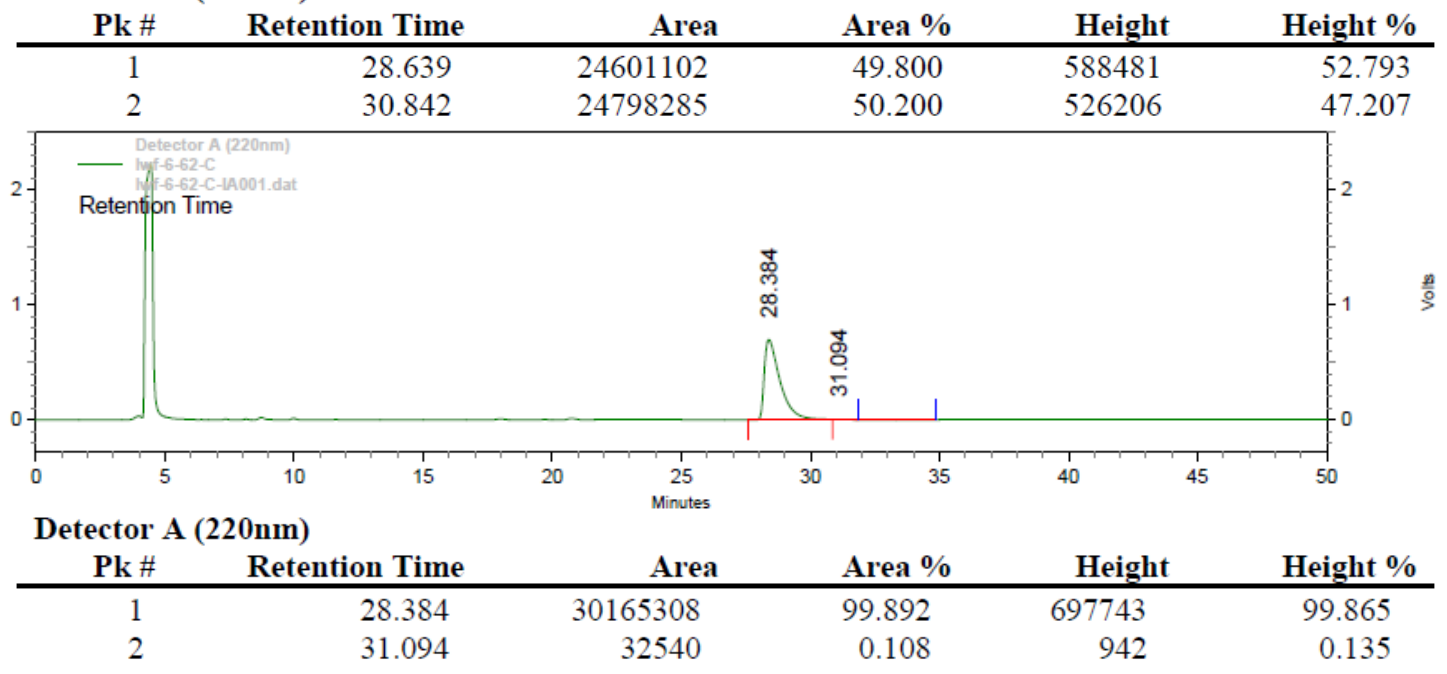

(4e) Table 3, entry 5

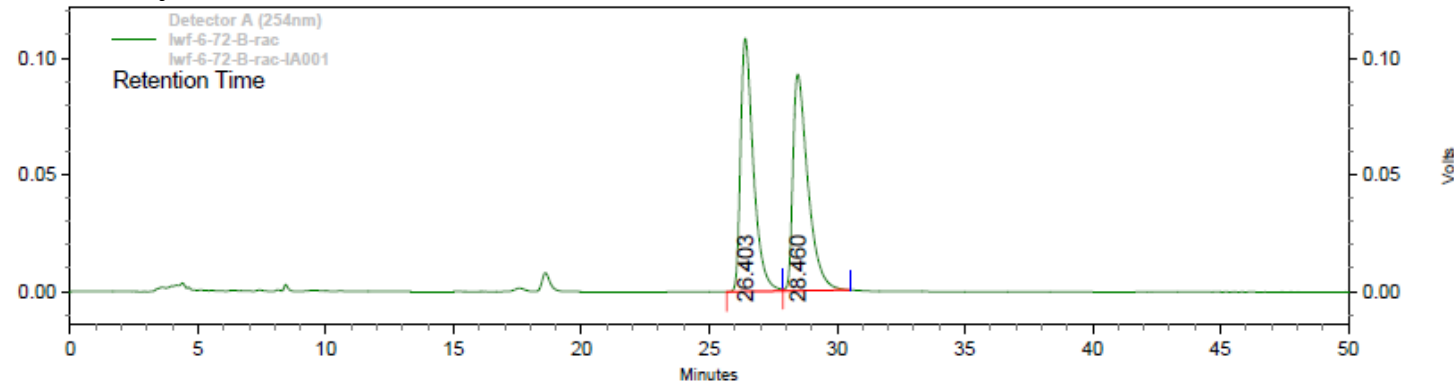

Detector A (220nm)

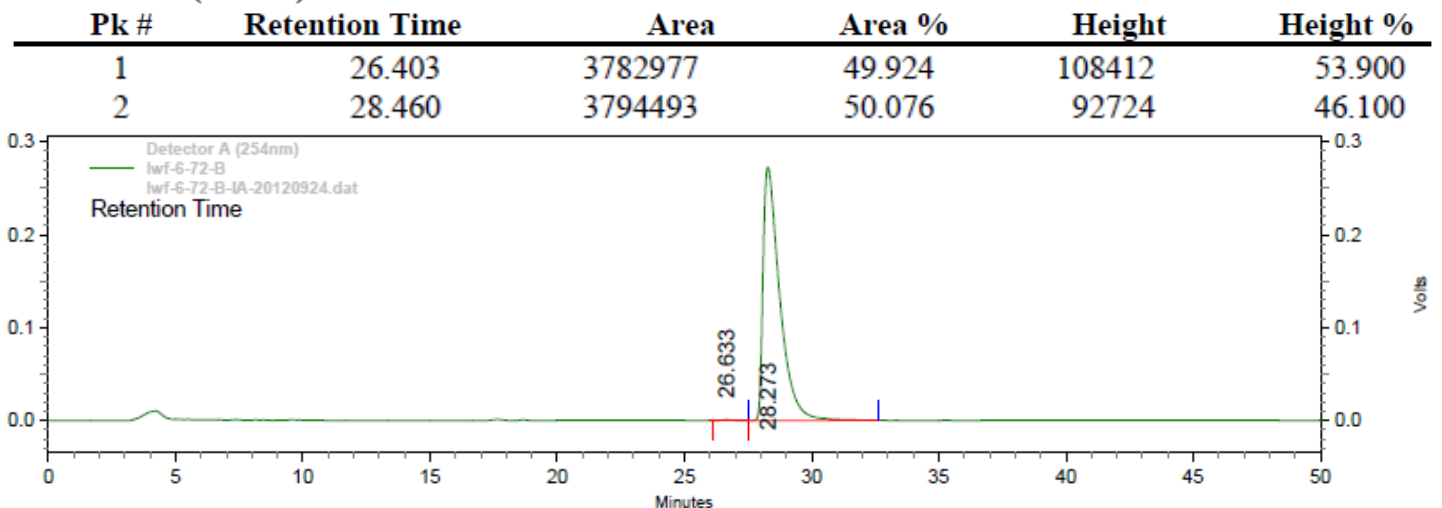

Detector A (220nm)

\begin{tabular}{cccrrr} 
Pk\# & Retention Time & Area & Area \% & Height & Height \% \\
\hline 1 & 26.633 & 24165 & 0.194 & 704 & 0.258 \\
2 & 28.273 & 12419566 & 99.806 & 272534 & 99.742
\end{tabular}

(4f) Table 3, entry 6 


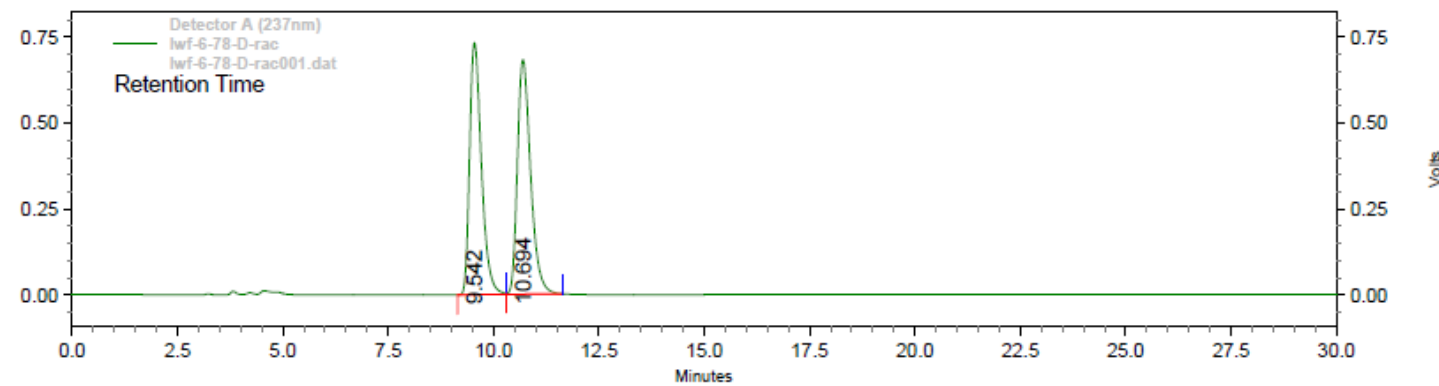

Detector A (220nm)

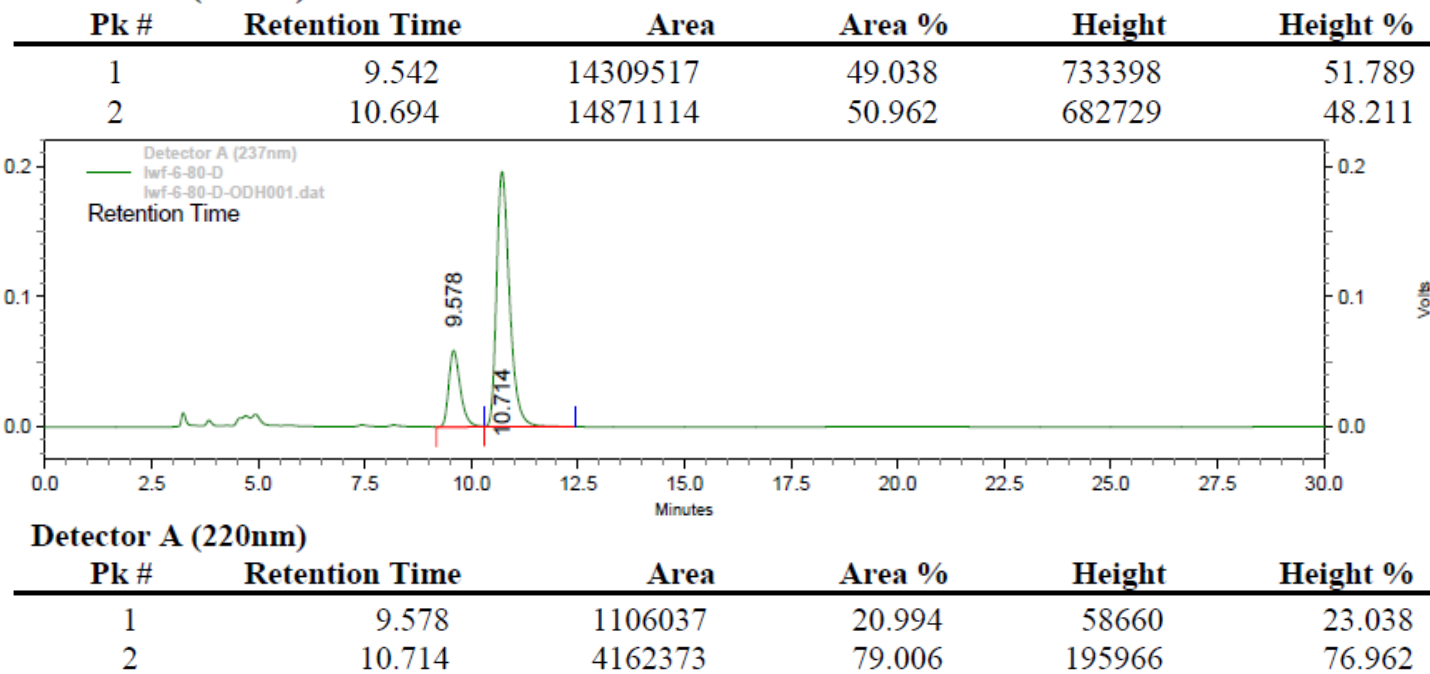

(4g) Table 3, entry 7

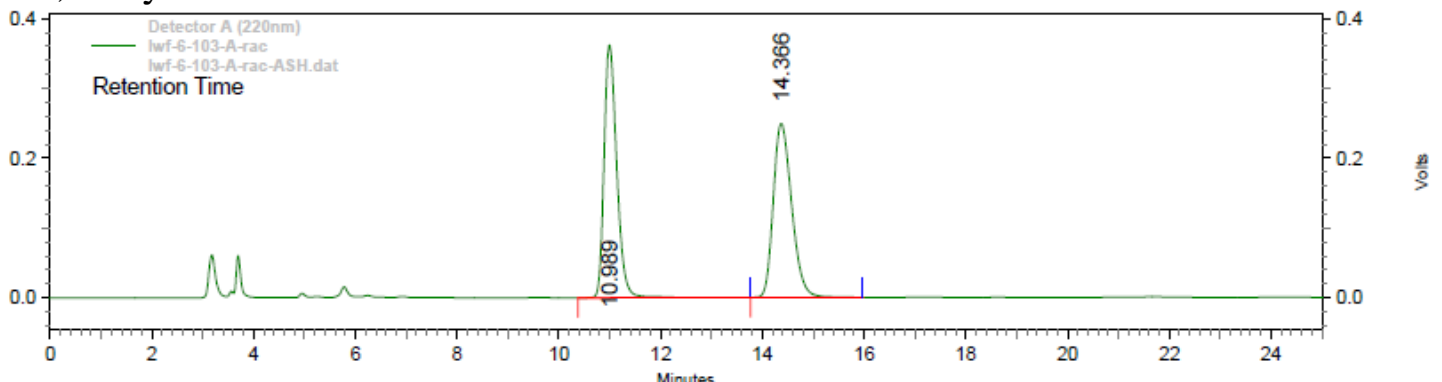

Detector A (220nm)

\begin{tabular}{cccccc} 
Pk \# & Retention Time & Area & Area \% & Height & Height \% \\
\hline 1 & 10.989 & 6265651 & 50.011 & 361903 & 59.236 \\
2 & 14.366 & 6262895 & 49.989 & 249052 & 40.764
\end{tabular}

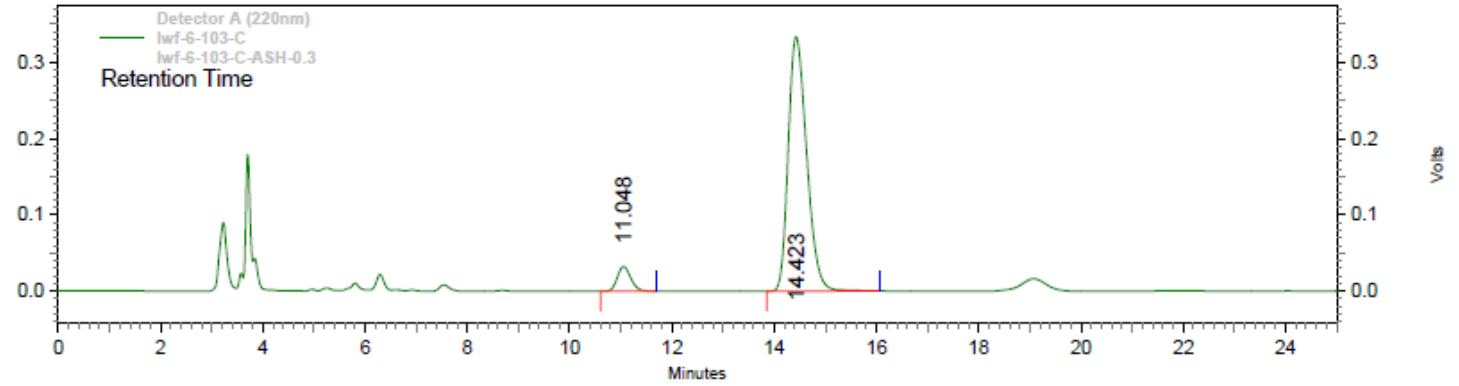

Detector A (220nm)

\begin{tabular}{ccccrr} 
Pk\# & Retention Time & Area & Area \% & Height & Height \% \\
\hline 1 & 11.048 & 594762 & 6.648 & 32455 & 8.870 \\
2 & 14.423 & 8351708 & 93.352 & 333456 & 91.130
\end{tabular}

(4h) Table 3, entry 8 


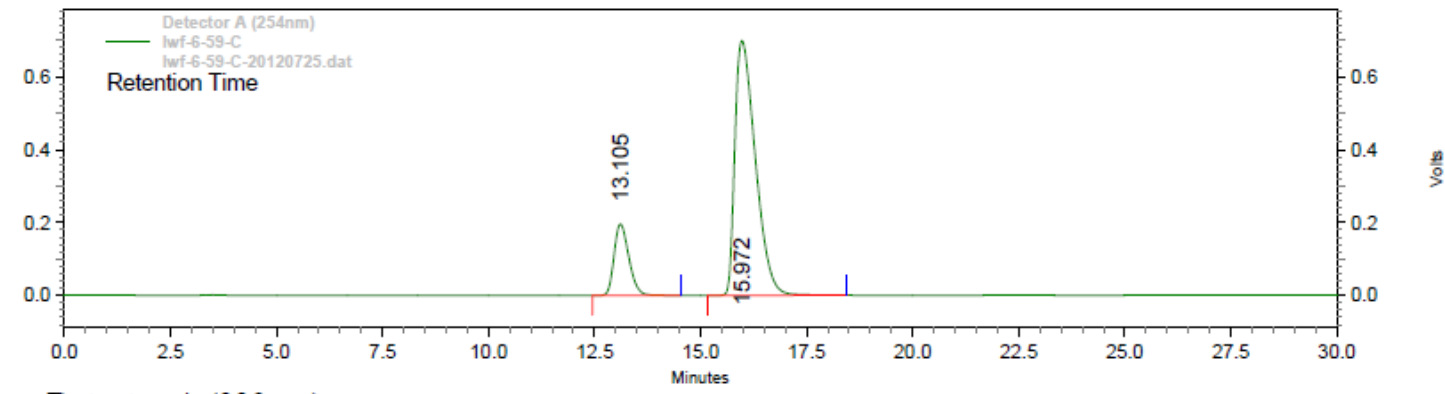

Detector A (220nm)

$\mathbf{P k}$ \#

Retention Time

Area

Area \%

Height

\begin{tabular}{lrrrrr} 
& \multicolumn{1}{c}{ Retention Time } & Area & Area \% & Height & Height \% \\
\cline { 2 - 6 } & 13.105 & 4742732 & 16.825 & 195375 & 21.880 \\
& 15.972 & 23445426 & 83.175 & 697563 & 78.120
\end{tabular}

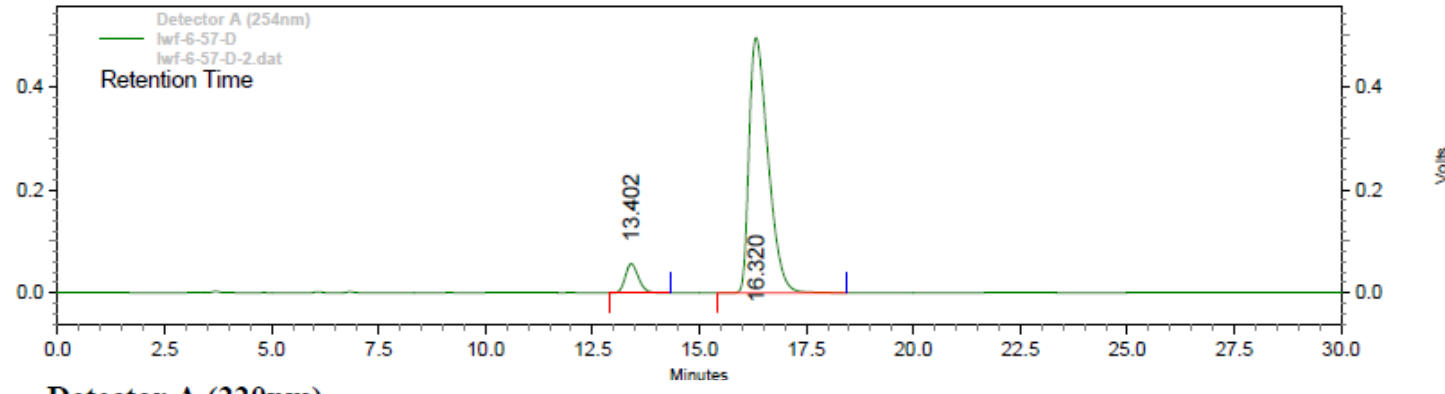

Detector A (220nm)

\begin{tabular}{cccccc} 
Pk \# & Retention Time & Area & Area \% & Height & Height \% \\
\hline 1 & 13.402 & 1209300 & 7.348 & 56477 & 10.248 \\
2 & 16.320 & 15248424 & 92.652 & 494607 & 89.752
\end{tabular}

(4i) Table 3, entry 9

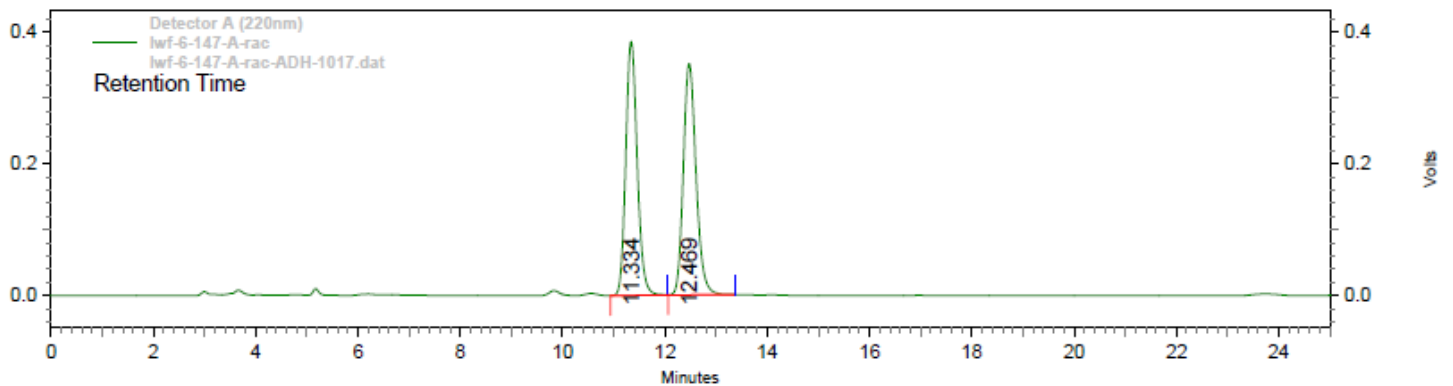

Detector A (220nm)

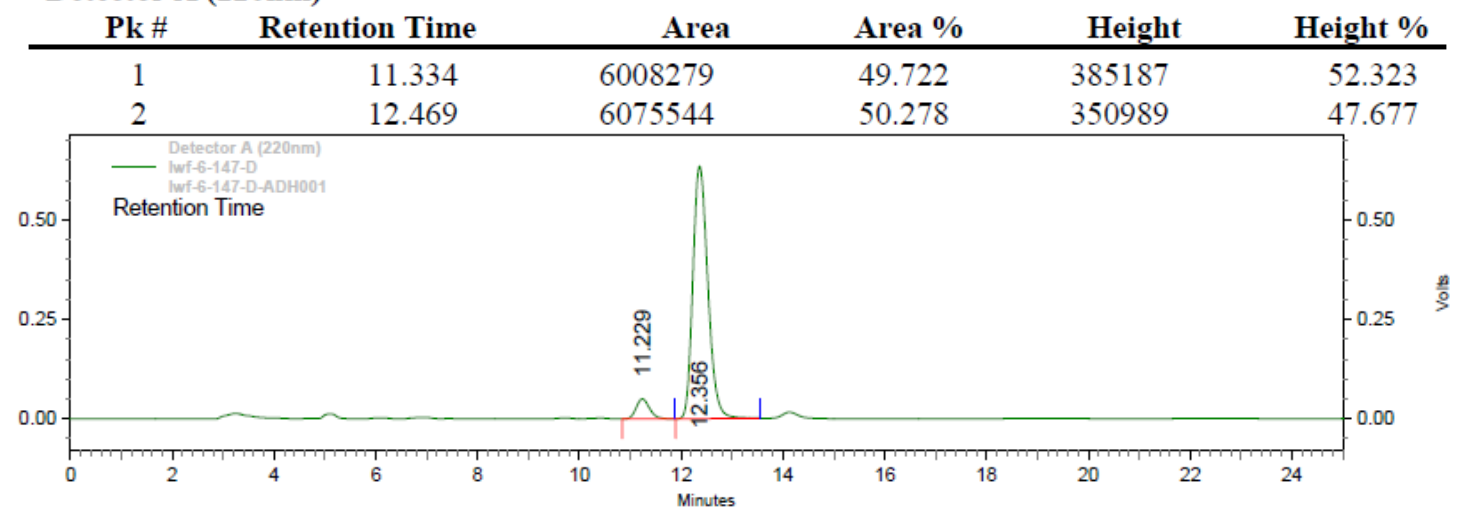




\begin{tabular}{|c|c|c|c|c|c|}
\hline \multicolumn{6}{|c|}{ Detector A (220nm) } \\
\hline $\mathbf{P k} \#$ & Retention Time & Area & Area \% & Height & Height \% \\
\hline 1 & 11.229 & 912839 & 6.550 & 50105 & 7.302 \\
\hline 2 & 12.356 & 13022587 & 93.450 & 636064 & 92.698 \\
\hline
\end{tabular}

(4j) Table 3, entry 10

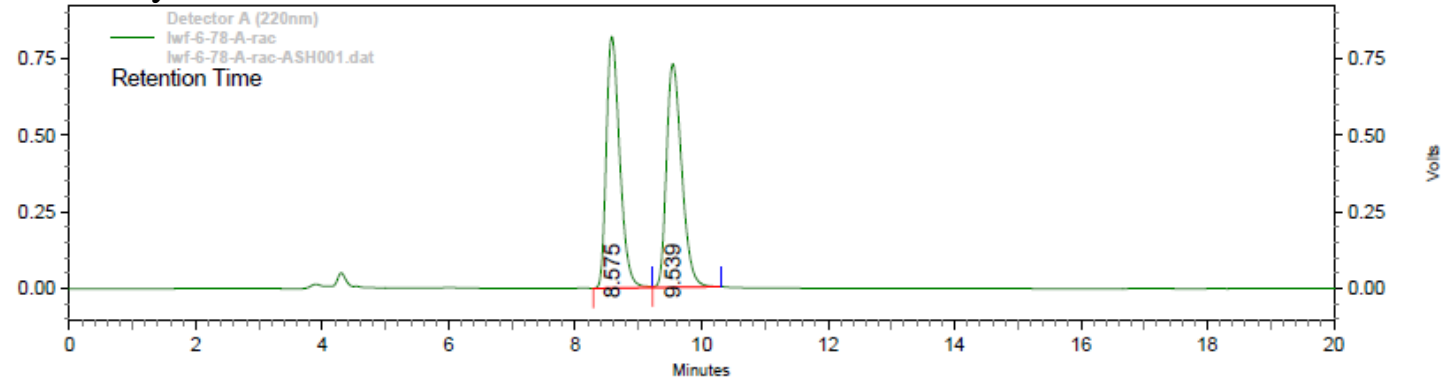

Detector A (220nm)

\begin{tabular}{cccccc} 
Pk \# & Retention Time & Area & Area \% & Height & Height \% \\
\hline 1 & 8.575 & 12103533 & 49.748 & 820800 & 52.953 \\
2 & 9.539 & 12226335 & 50.252 & 729255 & 47.047
\end{tabular}

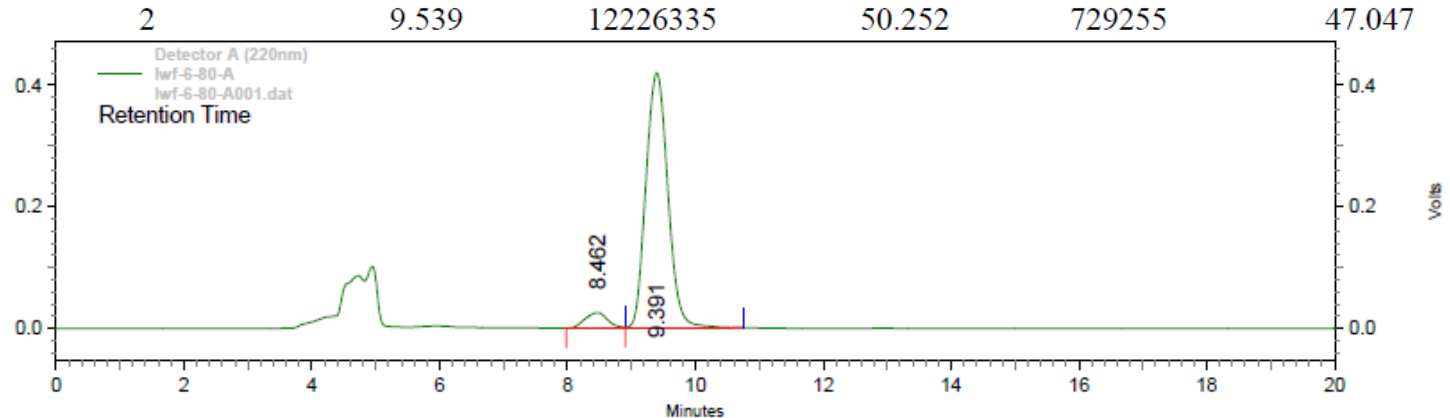

Detector A (220nm)

\begin{tabular}{cccccc} 
Pk\# & Retention Time & Area & Area \% & Height & Height \% \\
\hline 1 & 8.462 & 618778 & 5.819 & 25420 & 5.713 \\
2 & 9.391 & 10014896 & 94.181 & 419502 & 94.287
\end{tabular}

